Supporting Information

\title{
Why Silicon Doping Accelerates Electron Polaron Diffusion
}

\section{in Hematite}

\author{
Zhaohui Zhou, ${ }^{1}$ Run Long, ${ }^{2}$ Oleg V. Prezhdo ${ }^{3, *}$
}

${ }^{1}$ Chemical Engineering and Technology, School of Environmental Science and Engineering, Key Laboratory of Subsurface Hydrology and Ecological Effects in Arid Region, Ministry of Education, Chang'an University, Xi'an 710064, China

${ }^{2}$ College of Chemistry, Key Laboratory of Theoretical \& Computational Photochemistry of Ministry of Education, Beijing Normal University, Beijing 100875, China

${ }^{3}$ Deparment of Chemistry, University of Southern California, Los Angeles, CA 90089, USA 
Table S1. Average $\mathrm{Fe}^{2+}-\mathrm{O}$ bond length $\left(R_{\mathrm{Fe} 2+-\mathrm{O}}\right)$ and $\mathrm{Si}-\mathrm{O}$ bond length $\left(R_{\mathrm{Si}-\mathrm{O}}\right)$ derived from the static calculations on $\mathrm{Si} @ E P \mathrm{Fe}_{2} \mathrm{O}_{3}$, with respect to $R_{\mathrm{Fe} 2+-\mathrm{O}}$ of $2.11 \AA \AA$ in $\mathrm{e} @ \mathrm{EP}$ $\mathrm{Fe}_{2} \mathrm{O}_{3}$. Units are $\AA$.

\begin{tabular}{ccccccccc}
\hline & Fe1 & Fe2 & Fe3 & Fe4 & Fe5 & Fe6 & Fe7 & Fe8 \\
\hline$R_{\text {Fe2+-O }}$ & 2.135 & 2.123 & 2.135 & 2.135 & 2.128 & 2.128 & 2.127 & 2.131 \\
$R_{\mathrm{Si}-\mathrm{O}}$ & 1.849 & 1.855 & 1.849 & 1.849 & 1.851 & 1.851 & 1.851 & 1.847 \\
\hline
\end{tabular}


Table S2. Three neighboring $\mathrm{Fe}^{2+}$-Fe distances $\left(R_{1}, R_{2}\right.$, and $R_{3}$, units are $\AA$ ) extracted from the AIMD trajectory at $300 \mathrm{~K}$ for $\mathrm{e@EP} \mathrm{Fe}_{2} \mathrm{O}_{3}$, averaged over each stage defined in Figure 2, compared to the static values (in bold) at $0 \mathrm{~K}$.

\begin{tabular}{|c|c|c|c|c|c|c|c|c|c|c|c|}
\hline Stage & $R_{1}$ & $R_{2}$ & $R_{3}$ & Stage & $R_{1}$ & $R_{2}$ & $R_{3}$ & Stage & $R_{1}$ & $R_{2}$ & $R_{3}$ \\
\hline \multirow{3}{*}{ Fe1 } & Fe6 & Fe8 & Fe5 & \multirow{3}{*}{ Fe3 } & Fe6 & Fe7 & Fe8 & \multirow{3}{*}{ Fe6 } & Fe2 & Fe1 & Fe3 \\
\hline & & & & & & & & & & & \\
\hline & 2.91 & 2.97 & 2.99 & & 2.91 & 2.97 & 2.99 & & 2.91 & 2.97 & 2.99 \\
\hline \multirow{3}{*}{ Fe1-I } & $\mathrm{Fe} 5$ & Fe6 & Fe8 & \multirow{3}{*}{ Fe4 } & Fe5 & Fe8 & Fe7 & \multirow{3}{*}{ Fe6-I } & $\mathrm{Fe} 2$ & $\mathrm{Fe} 1$ & $\mathrm{Fe} 3$ \\
\hline & & & & & & & & & & & \\
\hline & 2.96 & 2.96 & 2.97 & & 2.91 & 2.97 & 2.99 & & 2.96 & 2.96 & 2.96 \\
\hline \multirow{3}{*}{ Fe1-II } & Fe6 & Fe8 & Fe5 & \multirow{3}{*}{ Fe4-I } & $\mathrm{Fe} 5$ & Fe8 & $\mathrm{Fe} 7$ & \multirow{3}{*}{ Fe6-II } & Fe1 & $\mathrm{Fe} 3$ & $\mathrm{Fe} 2$ \\
\hline & & & & & & & & & & & \\
\hline & 2.96 & 2.96 & 2.96 & & 2.96 & 2.96 & 2.96 & & 2.95 & 2.96 & 2.96 \\
\hline \multirow{3}{*}{ Fe1-III } & Fe6 & $\mathrm{Fe} 8$ & Fe5 & \multirow{3}{*}{ Fe5 } & $\mathrm{Fe} 2$ & Fe4 & Fe1 & \multirow{3}{*}{ Fe7 } & Fe4 & Fe2 & Fe3 \\
\hline & & & & & & & & & & & \\
\hline & 2.96 & 2.96 & 2.96 & & 2.91 & 2.97 & 2.99 & & 2.91 & 2.97 & 2.99 \\
\hline \multirow{3}{*}{ Fe2 } & Fe7 & Fe6 & Fe5 & \multirow{3}{*}{ Fe5-I } & $\mathrm{Fe} 4$ & Fe1 & $\mathrm{Fe} 2$ & \multirow{3}{*}{ Fe7-I } & $\mathrm{Fe} 4$ & $\mathrm{Fe} 2$ & Fe3 \\
\hline & & & & & & & & & & & \\
\hline & 2.91 & 2.97 & 2.99 & & 2.95 & 2.96 & 2.96 & & 2.96 & 2.96 & 2.96 \\
\hline \multirow{3}{*}{ Fe2-I } & Fe6 & Fe5 & Fe7 & \multirow{3}{*}{ Fe5-II } & Fe1 & $\mathrm{Fe} 2$ & $\mathrm{Fe} 4$ & \multirow{3}{*}{ Fe8 } & Fe1 & Fe4 & Fe3 \\
\hline & & & & & & & & & & & \\
\hline & 2.96 & 2.97 & 2.97 & & 2.95 & 2.96 & 2.96 & & 2.91 & 2.97 & 2.99 \\
\hline \multirow{3}{*}{ Fe2-II } & $\mathrm{Fe} 7$ & Fe6 & Fe5 & \multirow{3}{*}{ Fe5-III (1) } & $\mathrm{Fe} 4$ & $\mathrm{Fe} 2$ & $\mathrm{Fe} 1$ & \multirow{3}{*}{ Fe8-I } & $\mathrm{Fe} 1$ & $\mathrm{Fe} 4$ & $\mathrm{Fe} 3$ \\
\hline & & & & & & & & & & & \\
\hline & 2.96 & 2.96 & 2.96 & & 2.75 & 2.92 & 3.02 & & 2.96 & 2.96 & 2.96 \\
\hline
\end{tabular}

(1) an exception occurs in Fe5-III where the collected data are too few due to a very short duration of the EP stays in this stage. 
Table S3. Three neighboring $\mathrm{Fe}^{2+}$-Fe distances $\left(R_{1}, R_{2}\right.$, and $R_{3}$, units are $\AA$ ) extracted from the AIMD trajectory at $300 \mathrm{~K}$ for $\mathrm{Si} @ \mathrm{EP} \mathrm{Fe}_{2} \mathrm{O}_{3}$, time-averaged over each stage defined in Figure 3, and compared to the static values (in bold) at $0 \mathrm{~K}$.

\begin{tabular}{|c|c|c|c|c|c|c|c|c|c|c|c|}
\hline Stage & $R_{1}$ & $R_{2}$ & $R_{3}$ & Stage & $R_{1}$ & $R_{2}$ & $R_{3}$ & Stage & $R_{1}$ & $R_{2}$ & $R_{3}$ \\
\hline \multirow{3}{*}{ Fe8 } & Fe4 & $\mathrm{Fe} 3$ & Fe1 & \multirow{3}{*}{ Fe7 } & Fe4 & Fe3 & $\mathrm{Fe} 2$ & \multirow{3}{*}{ Fe3-III } & Fe8 & $\mathrm{Fe} 7$ & Fe6 \\
\hline & & & & & & & & & & & \\
\hline & 2.89 & 2.95 & 2.98 & & 2.90 & 2.97 & 3.01 & & 2.90 & 2.97 & 3.01 \\
\hline \multirow{3}{*}{ Fe8-I } & $\mathrm{Fe} 1$ & $\mathrm{Fe} 3$ & $\mathrm{Fe} 4$ & \multirow{3}{*}{ Fe7-I } & Fe4 & $\mathrm{Fe} 3$ & $\mathrm{Fe} 2$ & \multirow{3}{*}{ Fe3-IV } & Fe8 & $\mathrm{Fe} 7$ & Fe6 \\
\hline & & & & & & & & & & & \\
\hline & 2.94 & 2.95 & 2.95 & & 2.92 & 2.96 & 3.02 & & 2.93 & 2.95 & 2.95 \\
\hline \multirow{3}{*}{ Fe8-II } & $\mathrm{Fe} 4$ & $\mathrm{Fe} 3$ & Fe1 & \multirow{3}{*}{ Fe7-II } & Fe4 & $\mathrm{Fe} 3$ & $\mathrm{Fe} 2$ & \multirow{3}{*}{ Fe4 } & Fe8 & Fe5 & Fe7 \\
\hline & & & & & & & & & & & \\
\hline & 2.94 & 2.95 & 2.95 & & 2.91 & 2.96 & 3.02 & & 2.91 & 2.95 & 2.98 \\
\hline \multirow{3}{*}{ Fe8-III } & $\mathrm{Fe} 4$ & $\mathrm{Fe} 3$ & $\mathrm{Fe} 1$ & \multirow{3}{*}{ Fe7-III } & $\mathrm{Fe} 4$ & $\mathrm{Fe} 3$ & $\mathrm{Fe} 2$ & \multirow{3}{*}{ Fe4-I } & Fe8 & Fe5 & Fe7 \\
\hline & & & & & & & & & & & \\
\hline & 2.95 & 2.95 & 2.95 & & 2.92 & 2.96 & 3.02 & & 2.95 & 2.95 & 2.96 \\
\hline \multirow{3}{*}{ Fe8-IV } & $\mathrm{Fe} 4$ & $\mathrm{Fe} 3$ & Fe1 & \multirow{3}{*}{ Fe7-IV } & $\mathrm{Fe} 4$ & $\mathrm{Fe} 3$ & $\mathrm{Fe} 2$ & \multirow{3}{*}{ Fe4-II } & $\mathrm{Fe} 7$ & $\mathrm{Fe} 5$ & Fe8 \\
\hline & & & & & & & & & & & \\
\hline & 2.94 & 2.95 & 2.95 & & 2.91 & 2.96 & 3.01 & & 2.94 & 2.95 & 2.97 \\
\hline \multirow{3}{*}{ Fe5 } & Fe1 & Fe4 & Fe2 & \multirow{3}{*}{ Fe1 } & Fe8 & Fe6 & Fe5 & \multirow{3}{*}{ Fe4-III } & $\mathrm{Fe} 7$ & Fe5 & Fe8 \\
\hline & & & & & & & & & & & \\
\hline & 2.90 & 2.97 & 3.01 & & 2.91 & 2.95 & 2.98 & & 2.92 & 2.95 & 2.96 \\
\hline \multirow{3}{*}{ Fe5-I } & $\mathrm{Fe} 1$ & $\mathrm{Fe} 4$ & $\mathrm{Fe} 2$ & \multirow{3}{*}{ Fe1-I } & Fe6 & Fe8 & $\mathrm{Fe} 5$ & \multirow{3}{*}{ Fe4-IV } & $\mathrm{Fe} 7$ & $\mathrm{Fe} 5$ & Fe8 \\
\hline & & & & & & & & & & & \\
\hline & 2.93 & 2.96 & 3.01 & & 2.93 & 2.95 & 2.95 & & 2.92 & 2.95 & 2.98 \\
\hline \multirow{3}{*}{ Fe6 } & Fe3 & Fe1 & Fe2 & \multirow{3}{*}{ Fe3 } & Fe8 & Fe7 & Fe6 & \multirow{3}{*}{ Fe4-V } & Fe8 & $\mathrm{Fe} 5$ & $\mathrm{Fe} 7$ \\
\hline & & & & & & & & & & & \\
\hline & 2.90 & 2.97 & 3.01 & & 2.91 & 2.95 & 2.98 & & 2.94 & 2.94 & 2.96 \\
\hline \multirow{3}{*}{ Fe6-I } & $\mathrm{Fe} 3$ & $\mathrm{Fe} 1$ & $\mathrm{Fe} 2$ & \multirow{3}{*}{ Fe3-I } & Fe6 & $\mathrm{Fe} 7$ & Fe8 & \multirow{3}{*}{ Fe2 } & Fe7 & Fe6 & Fe5 \\
\hline & & & & & & & & & & & \\
\hline & 2.92 & 2.97 & 3.01 & & 2.93 & 2.93 & 2.95 & & 2.96 & 3.01 & 3.02 \\
\hline \multirow{3}{*}{ Fe6-II } & $\mathrm{Fe} 3$ & $\mathrm{Fe} 1$ & $\mathrm{Fe} 2$ & & Fe6 & $\mathrm{Fe} 7$ & Fe8 & & & & \\
\hline & & & & Fe3-II & & & & & & & \\
\hline & 2.92 & 2.96 & 3.01 & & 2.93 & 2.95 & 2.97 & & & & \\
\hline
\end{tabular}


Table S4. $\mathrm{Fe}^{2+}-\mathrm{O}$ bond lengths $\left(<R_{\mathrm{Fe} 2+-\mathrm{O}}>\right)$ time-averaged over the stages of $\mathrm{Fe}^{2+}$, and $\mathrm{Fe}^{3+}$-O bond lengths $\left(<R_{\mathrm{Fe} 3+-\mathrm{O}}>\right)$ time-averaged over the stages of $\mathrm{Fe}^{3+}$, derived from the AIMD simulations on e@EP and $\mathrm{Si} @ E P \mathrm{Fe}_{2} \mathrm{O}_{3}$, respectively, with the standard deviation in parentheses reflecting the magnitude of thermal fluctuations. Definition of the stages is shown in Figures 2 and 3, and units are $\AA$.

\begin{tabular}{|c|c|c|c|c|c|c|c|c|}
\hline & $\mathrm{Fe} 1$ & $\mathrm{Fe} 2$ & $\mathrm{Fe} 3$ & Fe4 & Fe5 & Fe6 & $\mathrm{Fe} 7$ & Fe8 \\
\hline \multicolumn{9}{|c|}{ e@EP Fe $\mathrm{F}_{3}$} \\
\hline \multirow{2}{*}{$R_{\mathrm{Fe} 2+-\mathrm{O}}$} & 2.118 & 2.118 & \multirow{2}{*}{ - } & 2.118 & 2.118 & 2.118 & 2.119 & 2.118 \\
\hline & $(0.022)$ & $(0.023)$ & & $(0.023)$ & $(0.023)$ & $(0.023)$ & $(0.023)$ & $(0.021)$ \\
\hline \multirow{2}{*}{$R_{\mathrm{Fe} 3+-\mathrm{O}}$} & 2.047 & 2.047 & 2.047 & 2.047 & 2.047 & 2.047 & 2.047 & 2.047 \\
\hline & $(0.019)$ & $(0.019)$ & (0.019) & (0.019) & (0.019) & $(0.019)$ & (0.019) & $(0.019)$ \\
\hline \multicolumn{9}{|c|}{$\mathrm{Si} @ E P \mathrm{Fe}_{2} \mathrm{O}_{3}$} \\
\hline \multirow{2}{*}{$R_{\mathrm{Fe} 2+-\mathrm{O}}$} & 2.128 & & 2.130 & 2.129 & 2.132 & 2.130 & 2.131 & 2.135 \\
\hline & $(0.029)$ & - & $(0.025)$ & $(0.027)$ & $(0.024)$ & $(0.022)$ & $(0.023)$ & $(0.023)$ \\
\hline \multirow{2}{*}{$R_{\mathrm{Fe} 3+-\mathrm{O}}$} & 2.059 & 2.053 & 2.060 & 2.060 & 2.059 & 2.059 & 2.060 & 2.058 \\
\hline & $(0.028)$ & $(0.020)$ & $(0.028)$ & $(0.028)$ & $(0.020)$ & $(0.019)$ & $(0.019)$ & $(0.019)$ \\
\hline
\end{tabular}




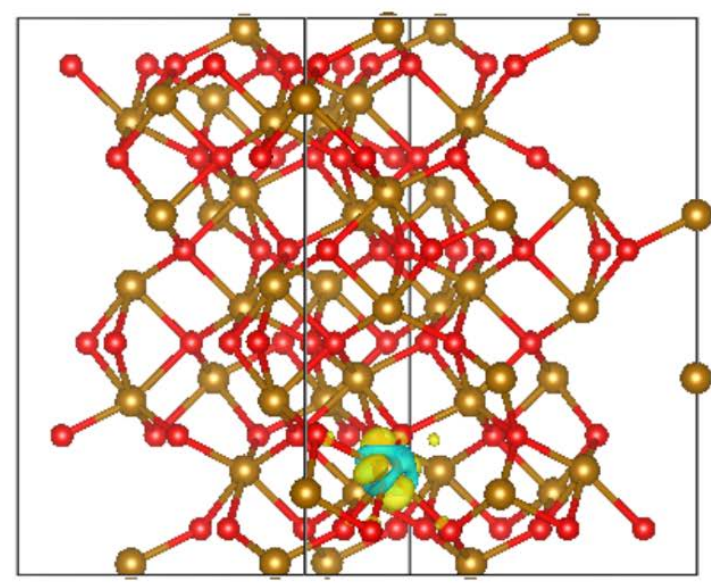

(a) $\mathrm{e} @ \mathrm{EP} \mathrm{Fe} \mathrm{O}_{3}$

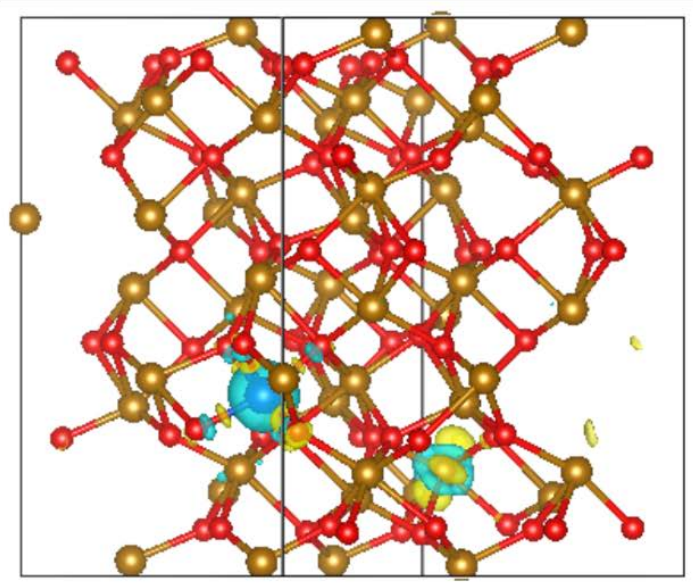

(b) $\mathrm{Si} @ \mathrm{EP} \mathrm{Fe} \mathrm{O}_{3}$

Figure S1. Difference charge density for the two EPs of $\mathrm{Fe}_{2} \mathrm{O}_{3}$ before and after an excess electron injection or a foreign Si atom doping, demonstrating that the EPs are generated in $\mathrm{Fe}_{2} \mathrm{O}_{3}$. Accumulation and depletion of electron charge are shown in yellow and cyan, respectively, with the iso-value of $0.01 \mathrm{e} / \mathrm{bohr}^{3}$. Charge depletion around the Si dopant in (b) arises from the different valence electrons for Si compared to Fe, namely, Si $3 s^{2} 3 p^{2}$ and Fe $3 d^{6} 4 s^{2}$. 

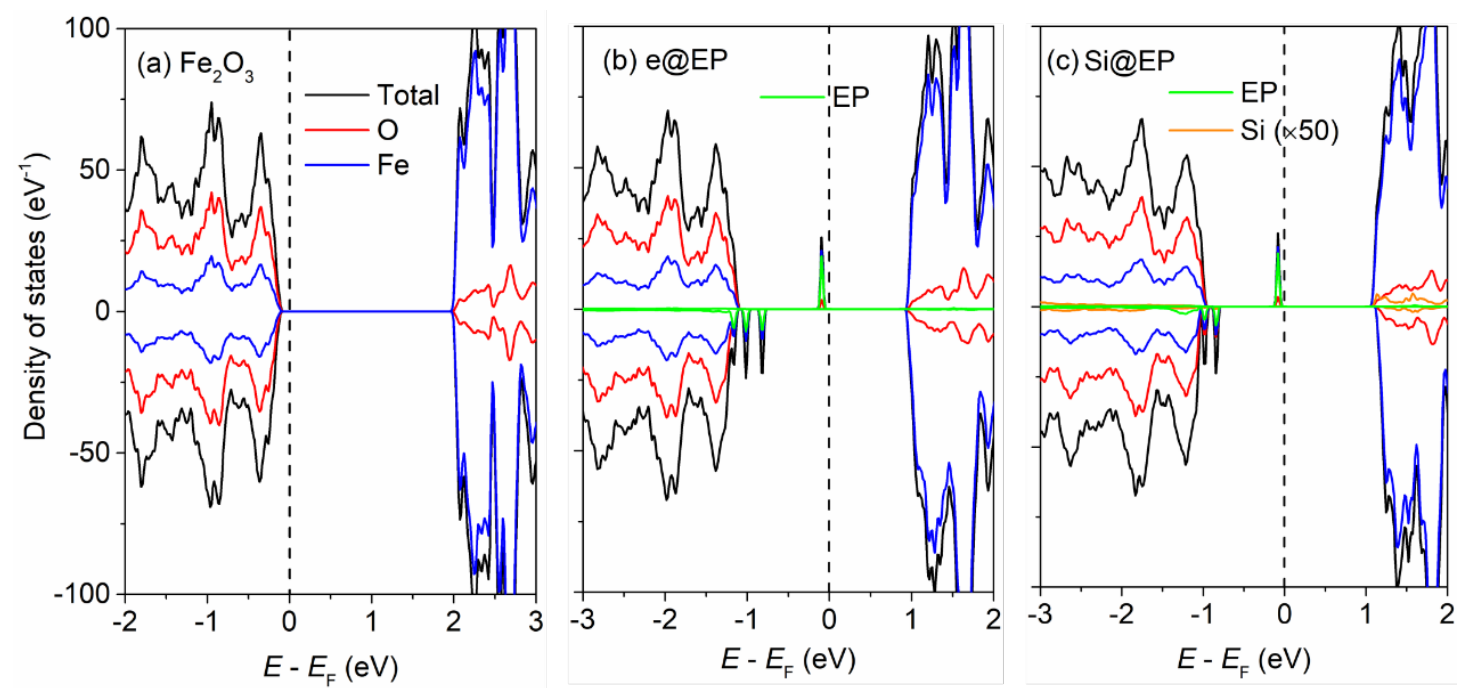

Figure S2. Density of states for (a) pure $\mathrm{Fe}_{2} \mathrm{O}_{3}$, (b) e@EP, and (c) Si@EP Fe2 $\mathrm{O}_{3}$. Formation of EPs in $\mathrm{Fe}_{2} \mathrm{O}_{3}$ gives rise to highly localized electronic states in the band gap. 


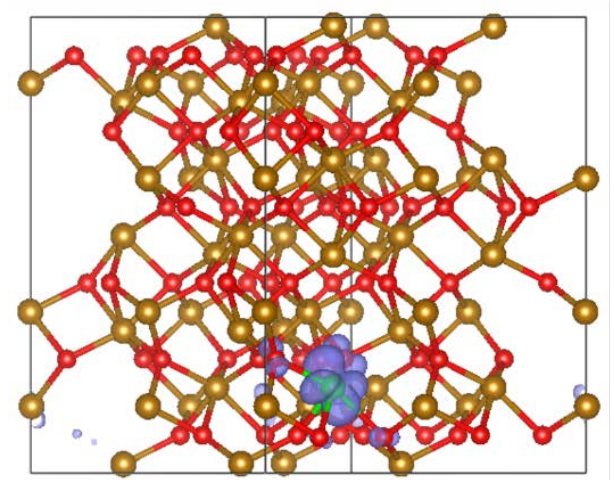

(a) e@EP $\mathrm{Fe}_{2} \mathrm{O}_{3}$

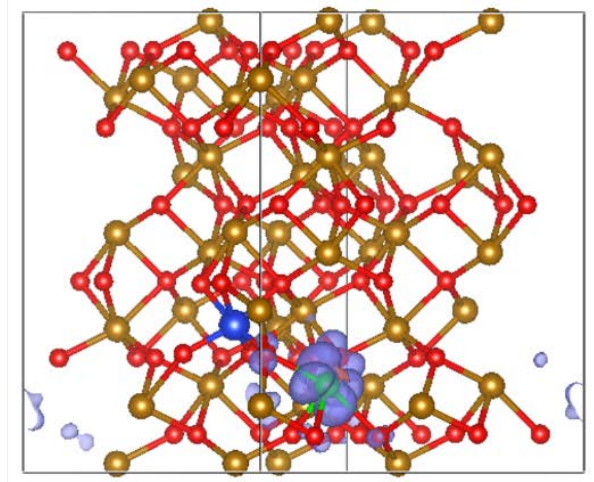

(b) Si@EP Fe2O3

Figure S3. Charge density of the EP state in (a) e@EP and (b) Si@EP Fe2 $\mathrm{O}_{3}$, depicted in dark blue with the iso-value of $0.002 \mathrm{e}^{\mathrm{bohr}}{ }^{3}$. 
(a) e@EP
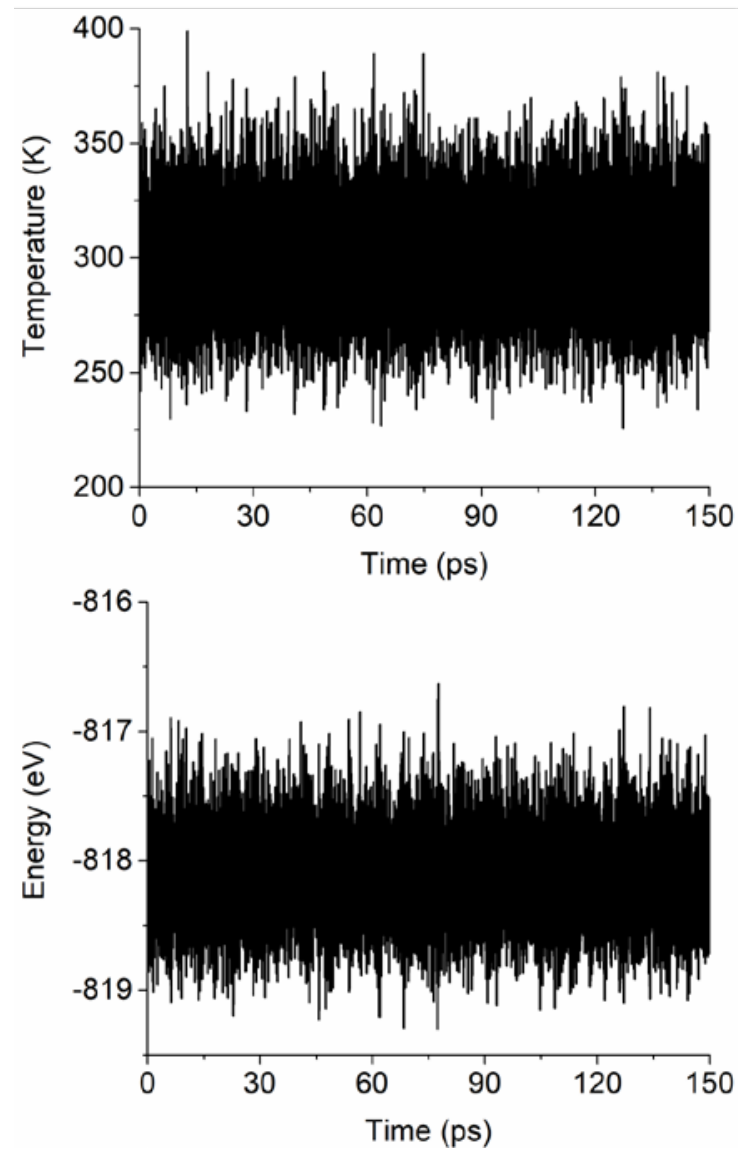

(b) Si@EP
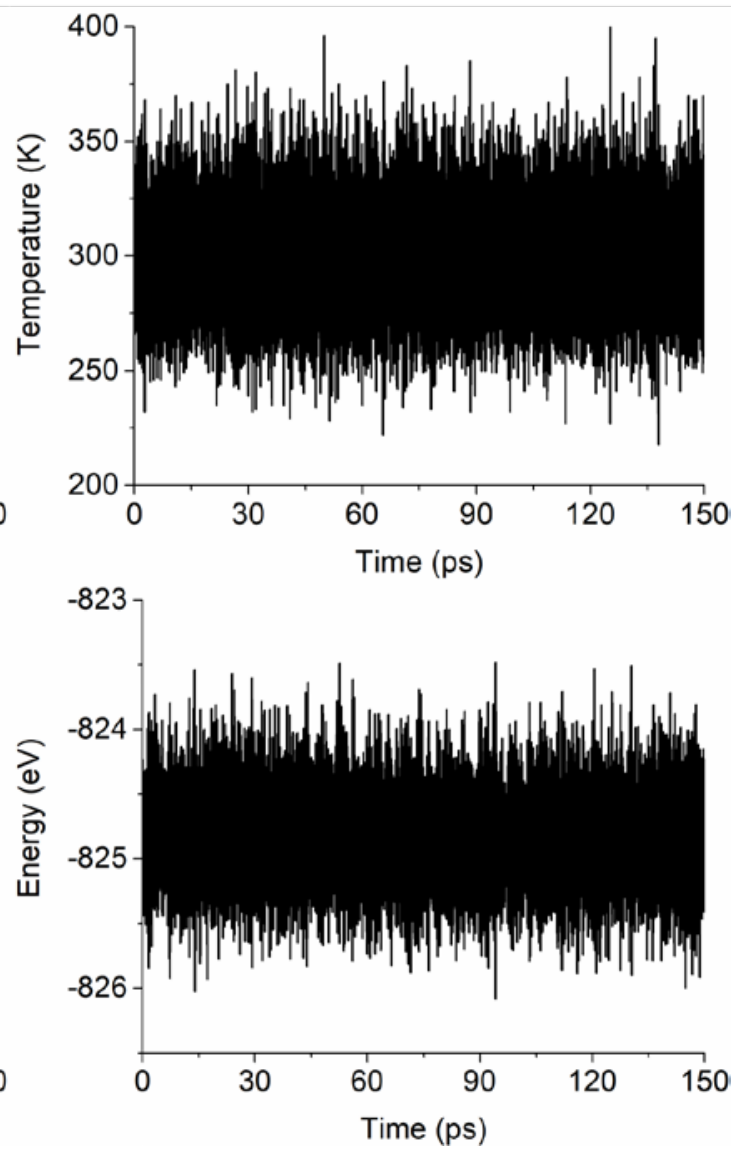

Figure S4. Temperature (upper panels) and energy (lower panels) as functions of time extracted from the AIMD trajectories for (a) e@EP and (b) Si@EP Fe $2 \mathrm{O}_{3}$. Note that the average energy of $\mathrm{Si} @ \mathrm{EP} \mathrm{Fe}_{2} \mathrm{O}_{3}$ is lower by $\sim 43 \mathrm{meV}$ after 55 ps. 


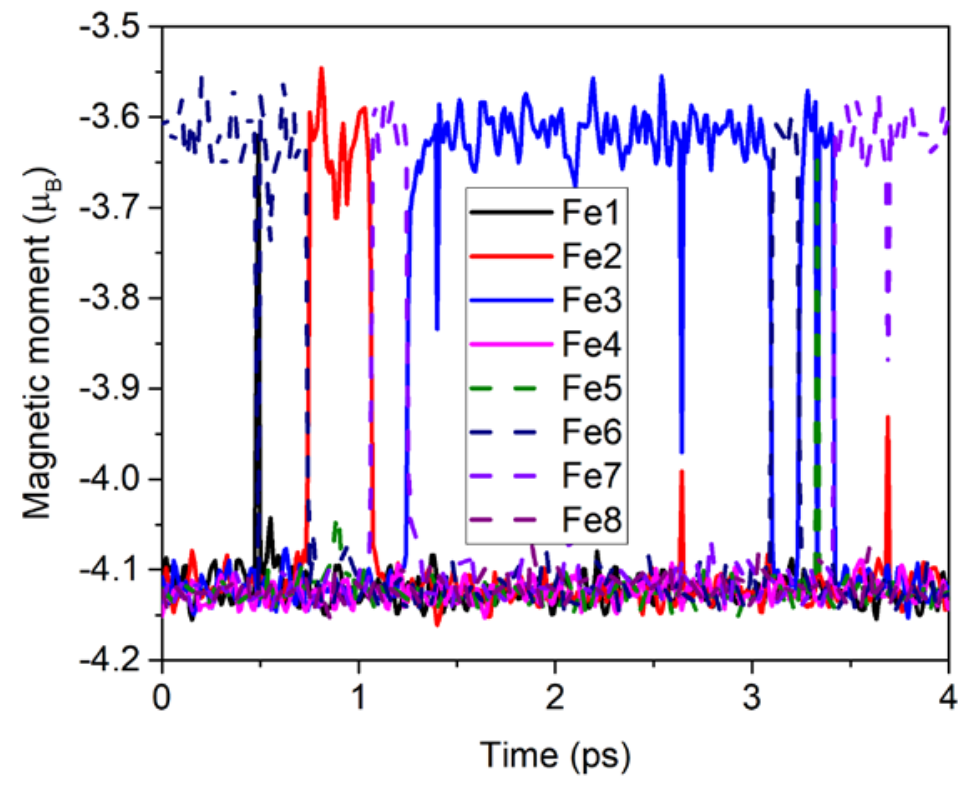

Figure S5. Local magnetic moments on the eight Fe ions of the Fe bilayer in the basal plane for e@EP $\mathrm{Fe}_{2} \mathrm{O}_{3}$ at $600 \mathrm{~K}$ as functions of the simulation time. The EP hops 9 times during the simulation time of 4 ps, significantly faster than the EP at $300 \mathrm{~K}$. 


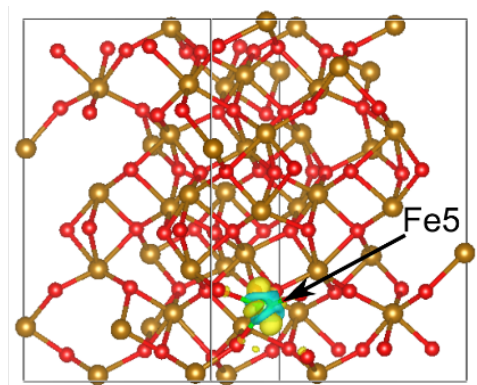

$5 \mathrm{ps}$

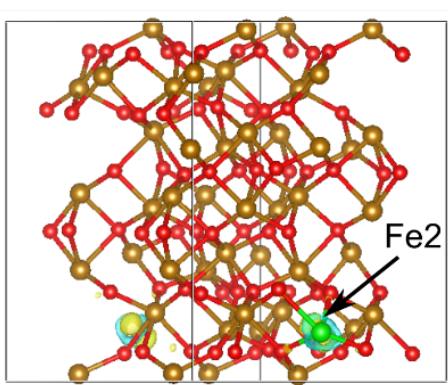

$9.5 \mathrm{ps}$

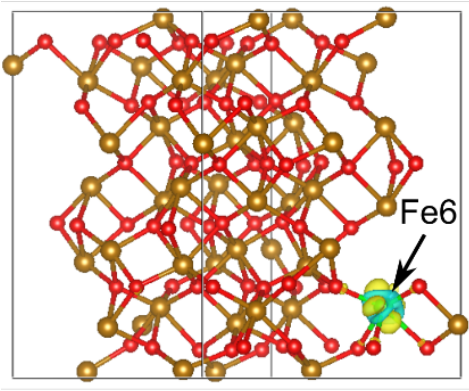

25 ps

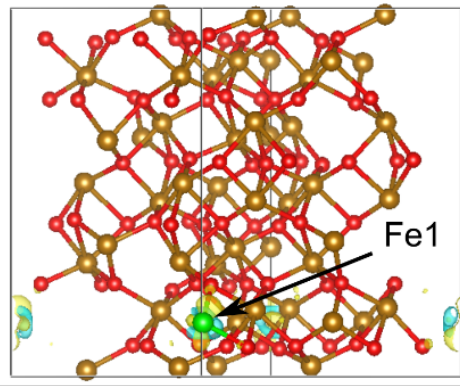

$37.5 \mathrm{ps}$

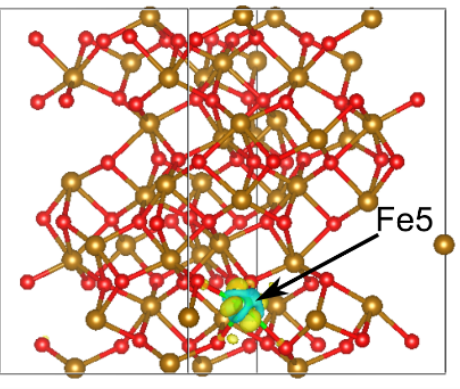

40 ps

Figure S6. Difference charge density for snapshots chosen from the first 40 ps of the 150 ps trajectory for e@EP $\mathrm{Fe}_{2} \mathrm{O}_{3}$ at $300 \mathrm{~K}$, depicted with the iso-value of $0.01 \mathrm{e} / \mathrm{bohr}^{3}$. 


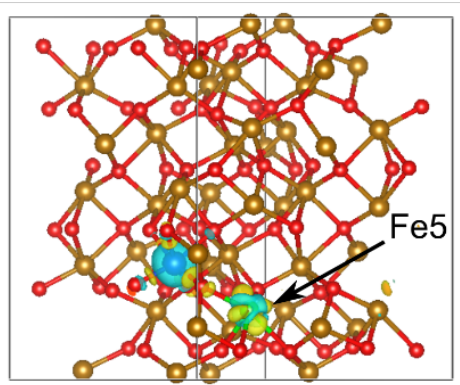

$2.5 \mathrm{ps}$

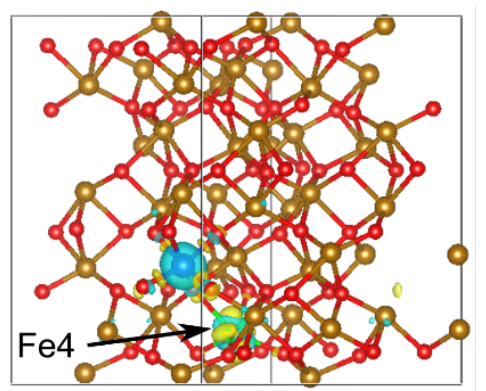

12.7 ps

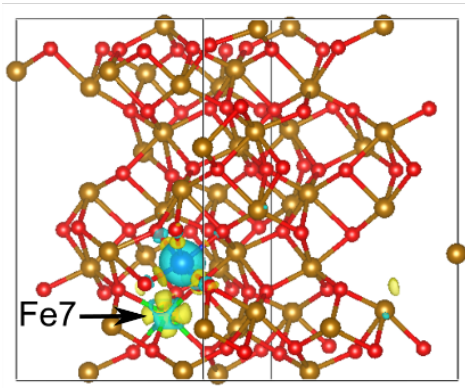

20 ps

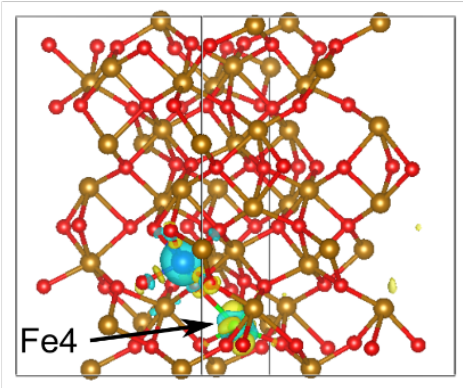

30 ps

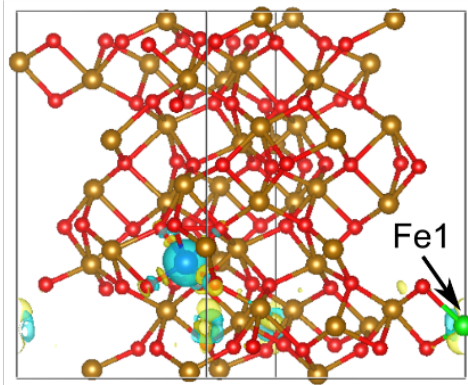

5 ps

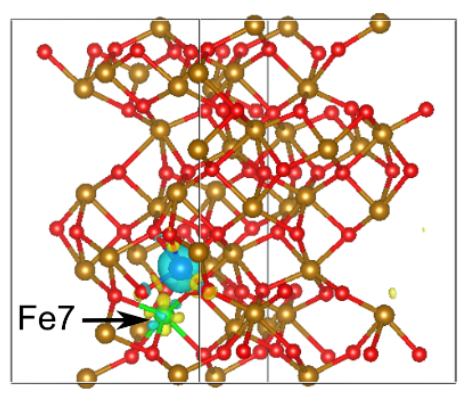

15.5 ps

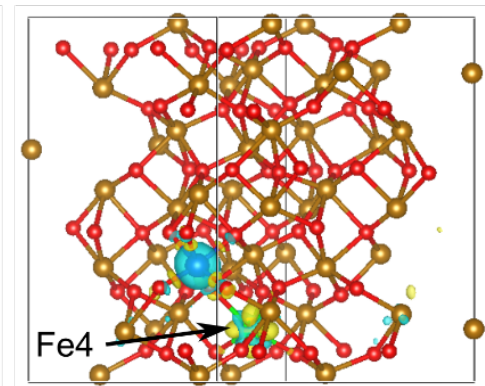

$22.5 \mathrm{ps}$

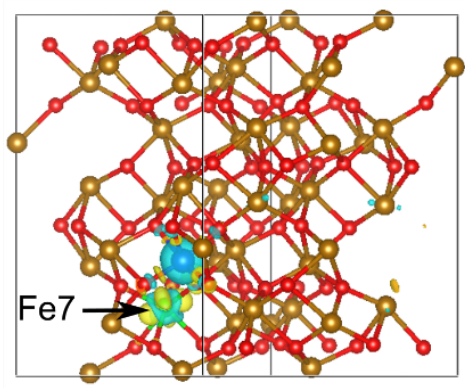

31 ps

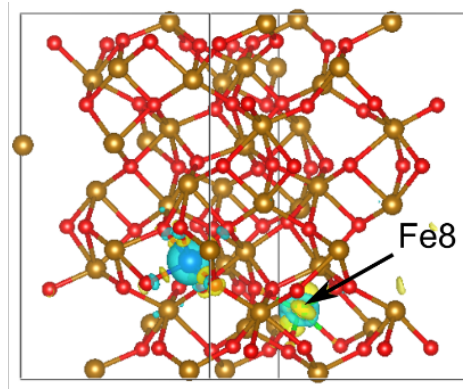

$10 \mathrm{ps}$

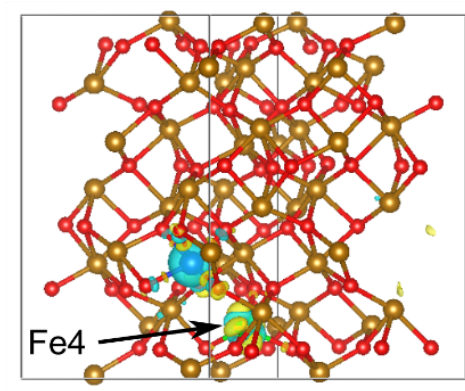

18.5 ps

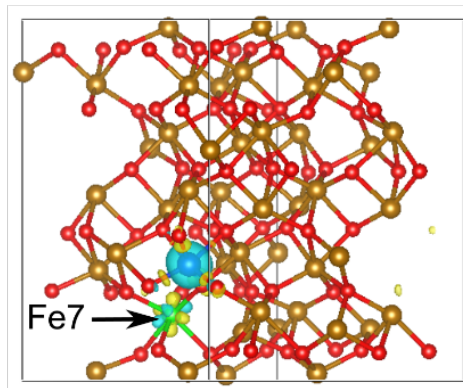

25.5 ps

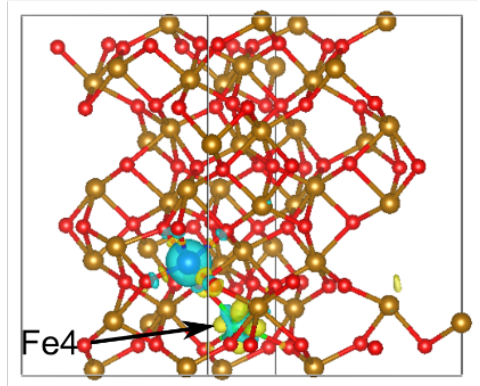

$32.1 \mathrm{ps}$

Figure S7. Difference charge density for snapshots chosen from the first 35 ps of the 150 ps trajectory for $\mathrm{Si} @ \mathrm{EP} \mathrm{Fe}_{2} \mathrm{O}_{3}$ at $300 \mathrm{~K}$, depicted with the iso-value of 0.01 e/bohr ${ }^{3}$. 
(a)

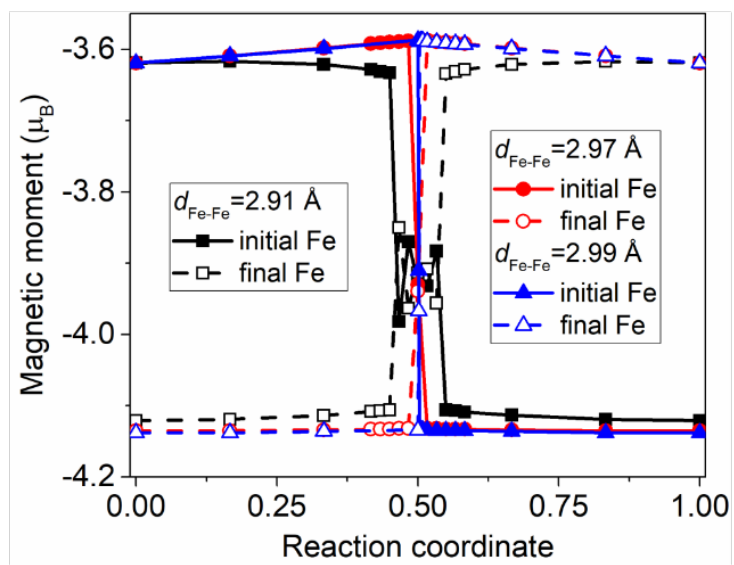

(b)

(c)
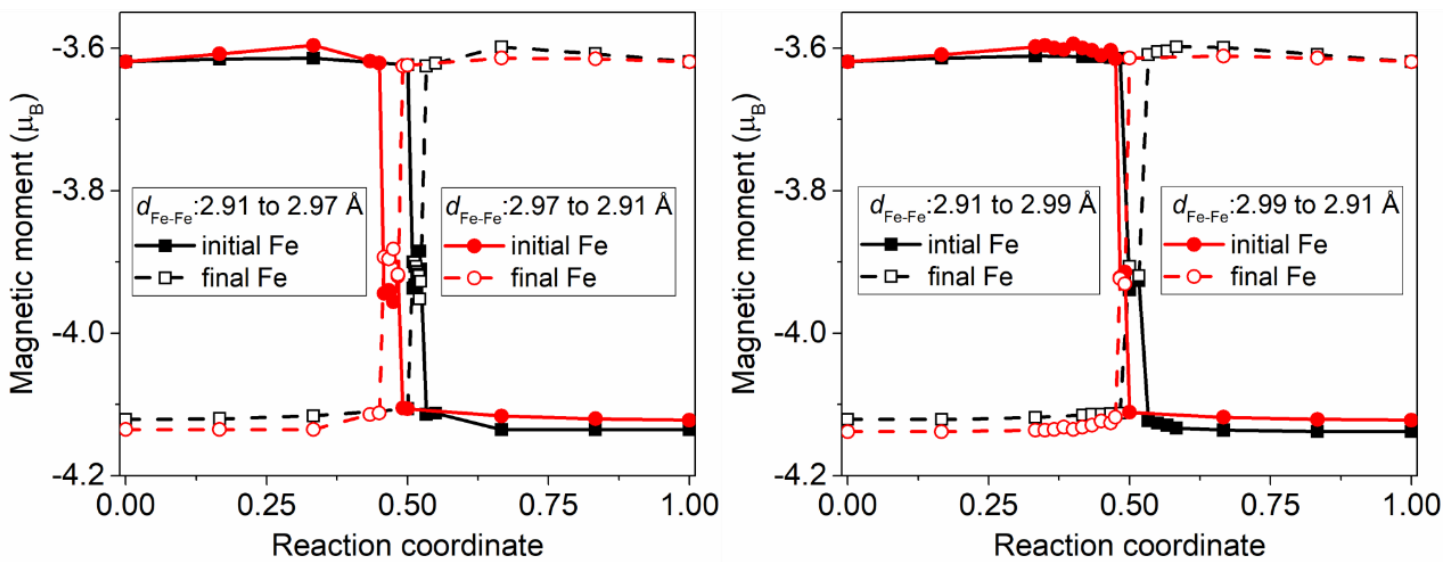

Figure S8. Local magnetic moment on the two Fe ions which are involved in the EP hopping along the reaction path in e@EP $\mathrm{Fe}_{2} \mathrm{O}_{3}$ between (a) equivalent and $(\mathbf{b}, \mathbf{c})$ inequivalent initial and final coordinates. The reaction path is defined by Equation (3). 
(a)

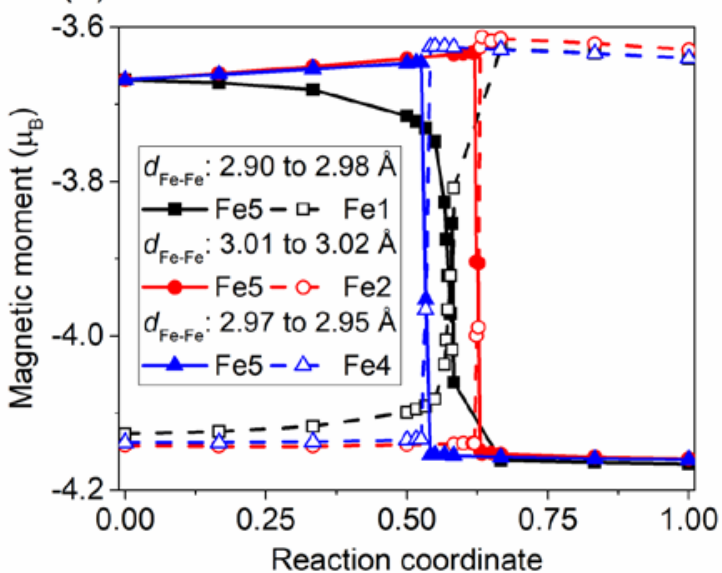

(c)

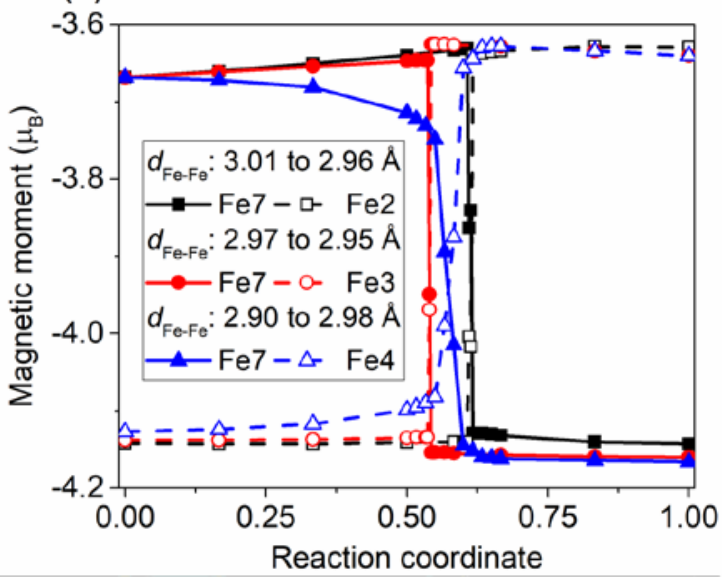

(b)

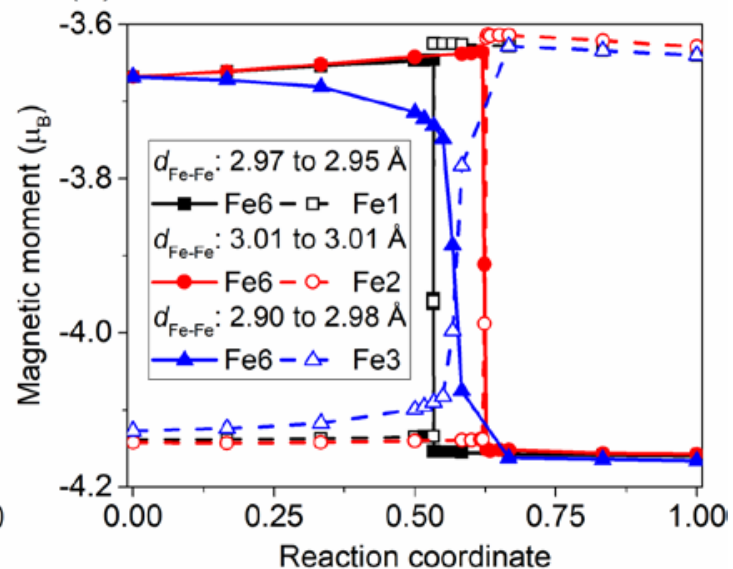

(d)

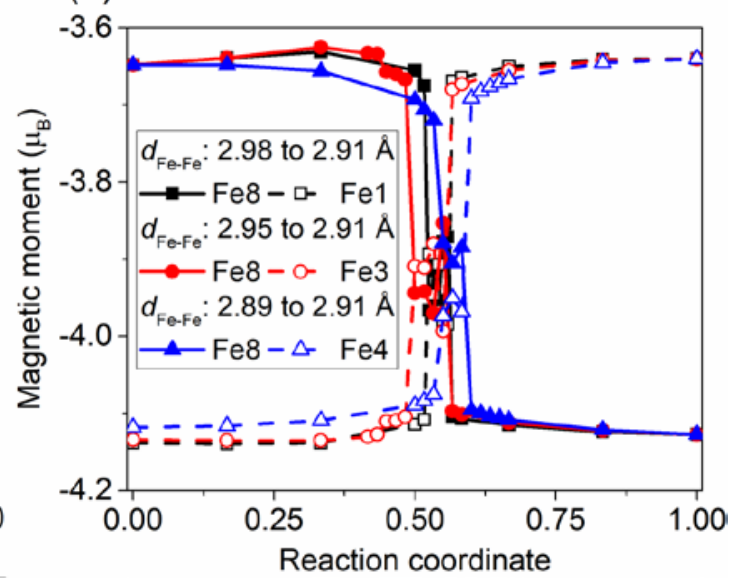

Figure S9. Local magnetic moments on the two Fe ions in $\mathrm{Si} @ \mathrm{EP} \mathrm{Fe}_{2} \mathrm{O}_{3}$ which are involved in the EP transfer along the reaction path defined by Equation (3), with the initial coordinate on (a) Fe5, (b) Fe6, (c) Fe7 and (d) Fe8, and the final coordinate on their three nearest Fe neighbors. 
(a)
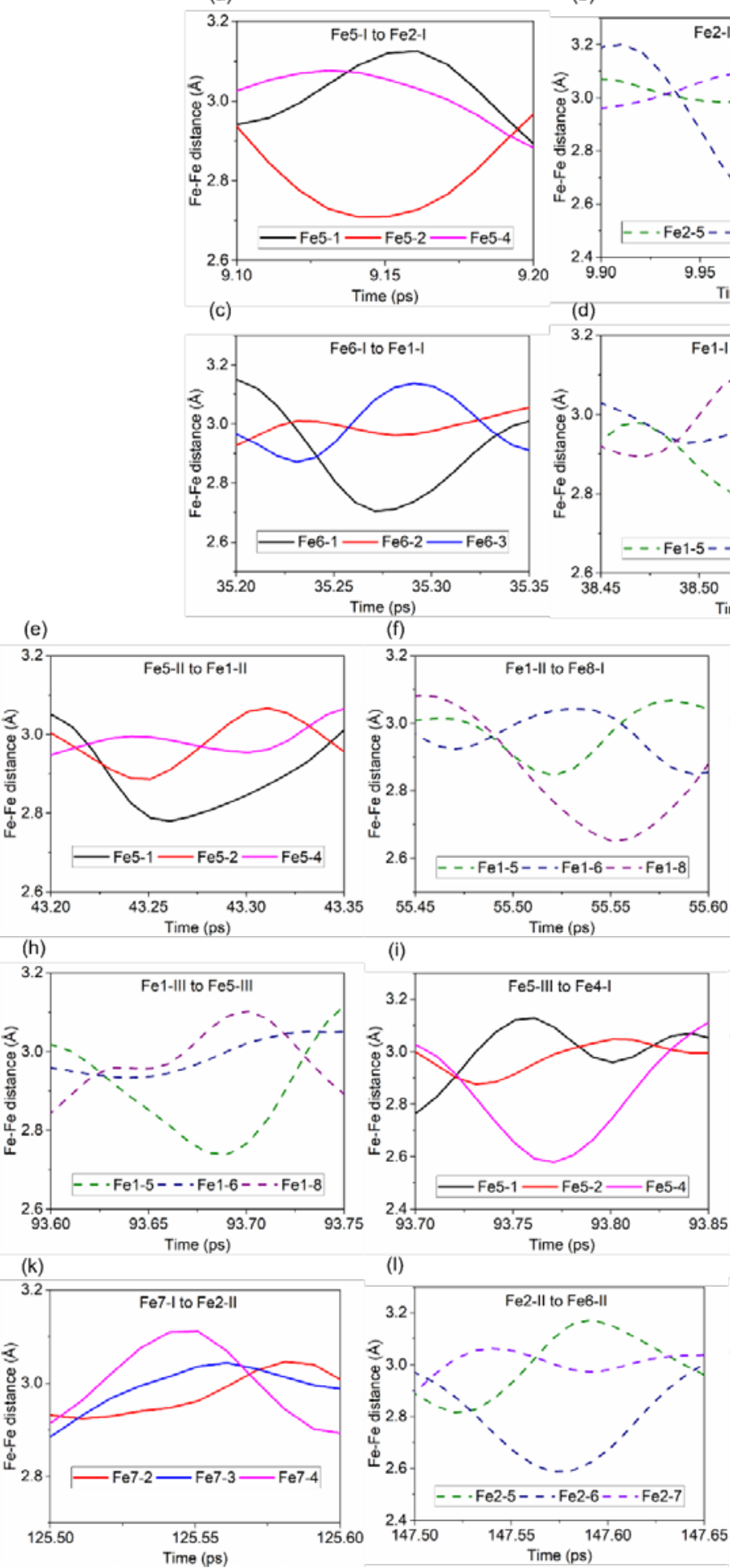

(b)
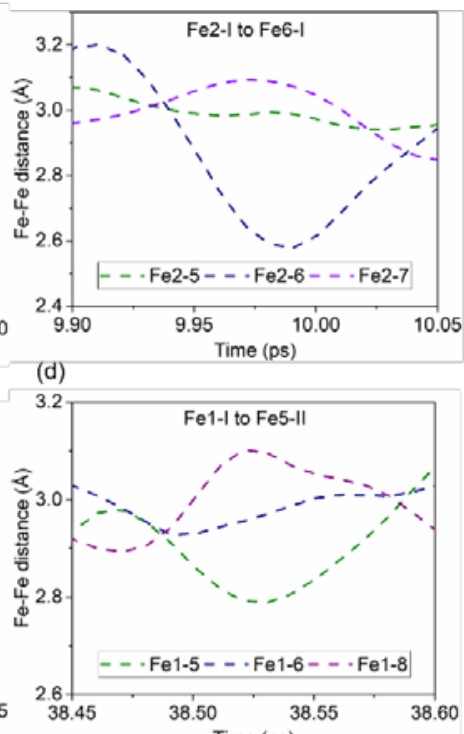

(g)
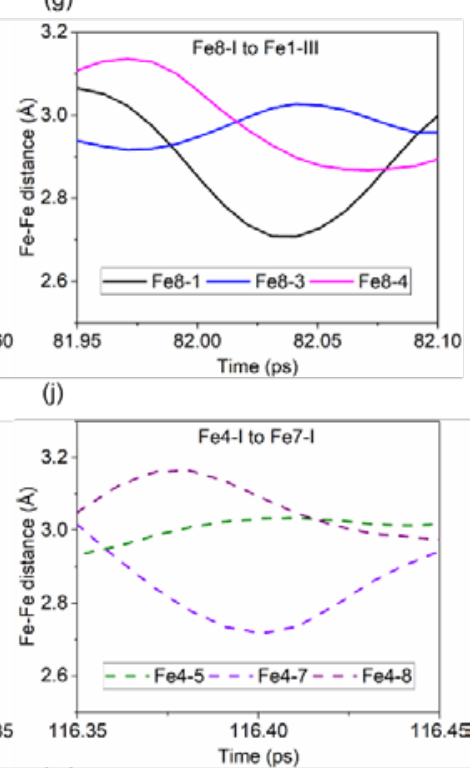

(m)

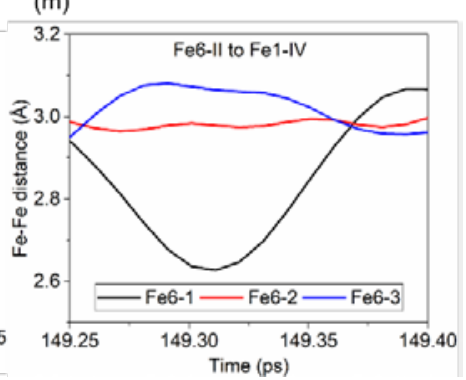

Figure S10. Three nearest Fe-Fe distances in the temporal windows of 0.1-0.15 ps for each EP hopping in e@EP $\mathrm{Fe}_{2} \mathrm{O}_{3}$, extracted from the AIMD trajectory of 150 ps: (a) Fe5-I to Fe2-I, (b) Fe2-I to Fe6-I, (c) Fe6-I to Fe1-I, (d) Fe1-I to Fe5-II, (e) Fe5-II to Fe1-II, (f) Fe1-II to Fe8-I, (g) Fe8-I to Fe1-III, (h) Fe1-III to Fe5-III, (i) Fe5-III to Fe4I, (j) Fe4-I to Fe7-I, (k) Fe7-I to Fe2-II, (l) Fe2-II to Fe6-II, and (m) Fe6-II to Fe1-IV. 

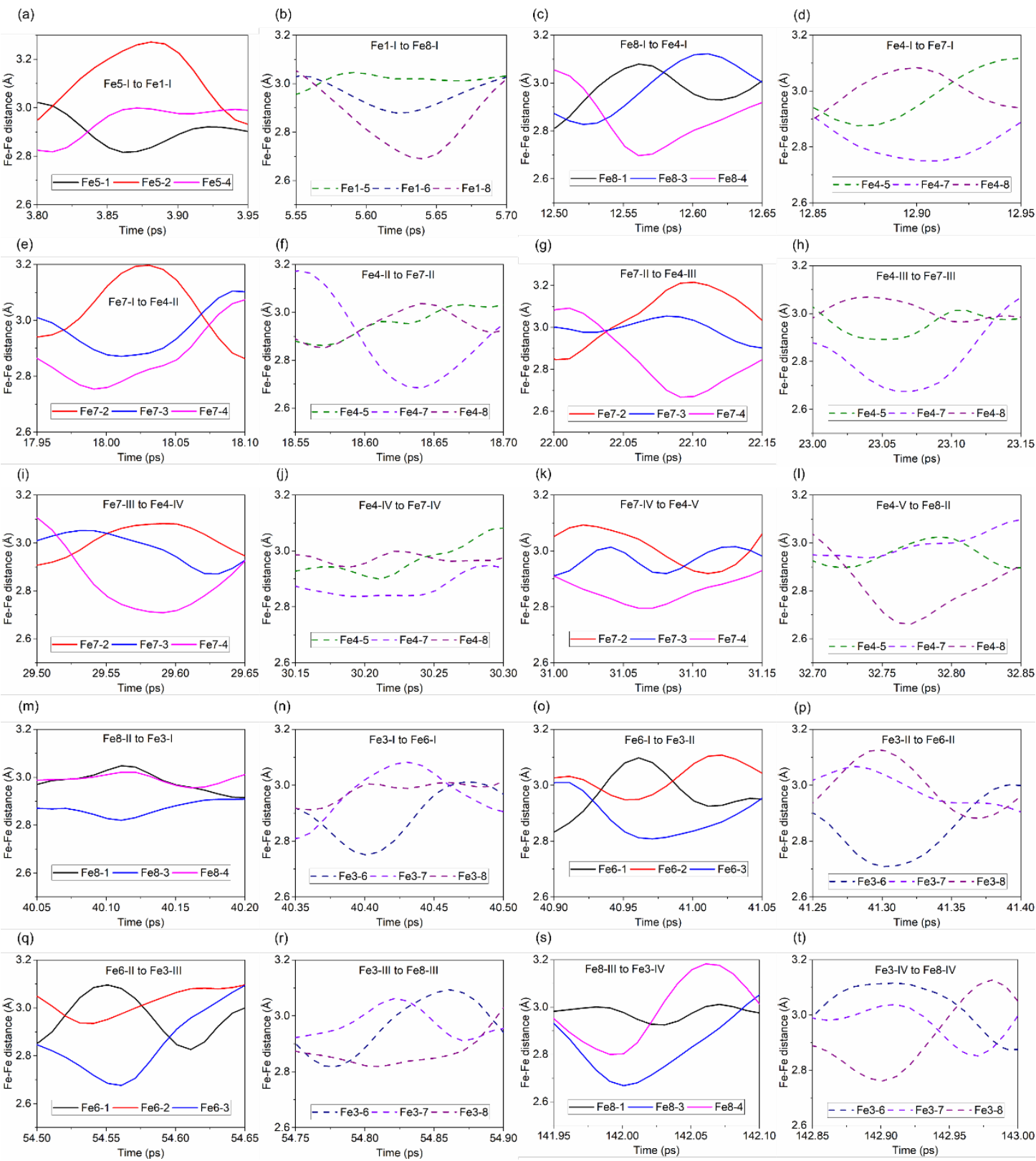

Figure S11. Three shortest Fe-Fe distances in the temporal windows of 0.1-0.15 ps for each EP hopping in $\mathrm{Si} @ E P \mathrm{Fe}_{2} \mathrm{O}_{3}$, extracted from the AIMD trajectory of 150 ps: (a) Fe5-I to Fe1-I, (b) Fe1-I to Fe8-I, (c) Fe8-I to Fe4-I, (d) Fe4-I to Fe7-I, (e) Fe7-I to Fe4-II, (f) Fe4-II to Fe7-II, (g) Fe7-II to Fe4-III, (h) Fe4-III to Fe7-III, (i) Fe7-III to Fe4-IV, (j) Fe4-IV to Fe7-IV, (k) Fe7-IV to Fe4-V, (l) Fe4-V to Fe8-II, (m) Fe8-II to Fe3-I, (n) Fe3-I to Fe6-I, (o) Fe6-I to Fe3-II, (p) Fe3-II to Fe6-II, (q) Fe6-II to Fe3-III, (r) Fe3-III to Fe8-III, (s) Fe8-III to Fe3-IV, and (t) Fe3-IV to Fe8-IV. 


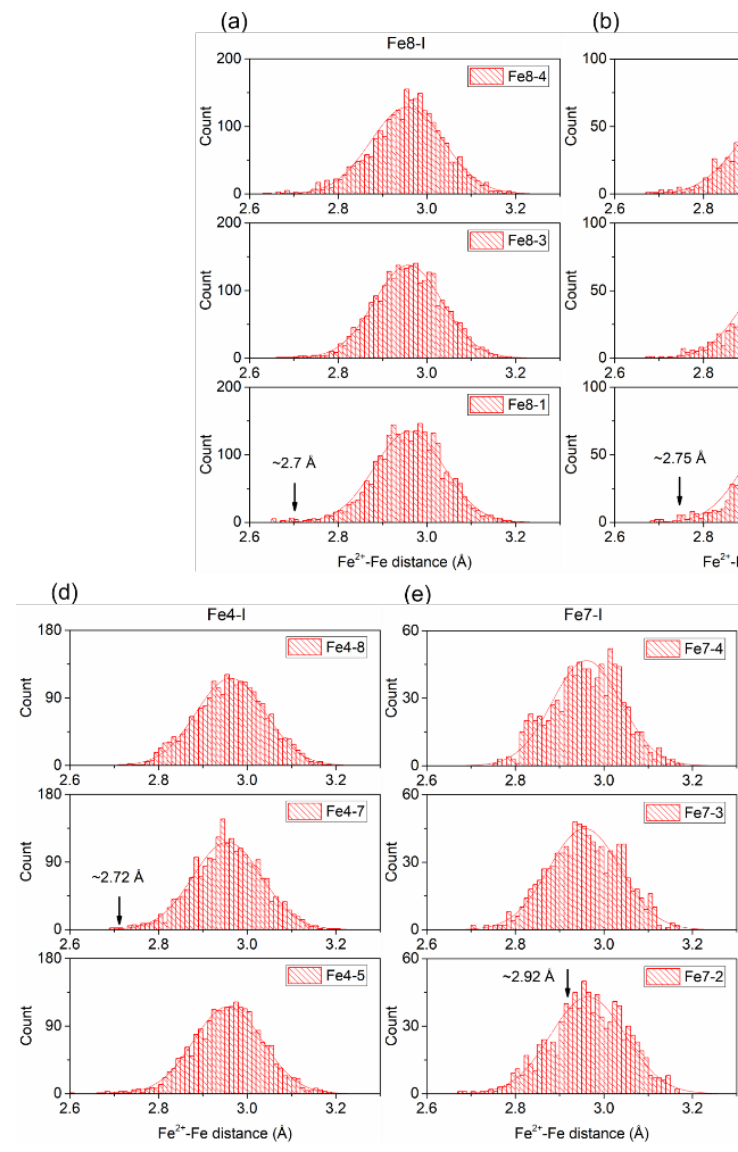

(b)

(c)
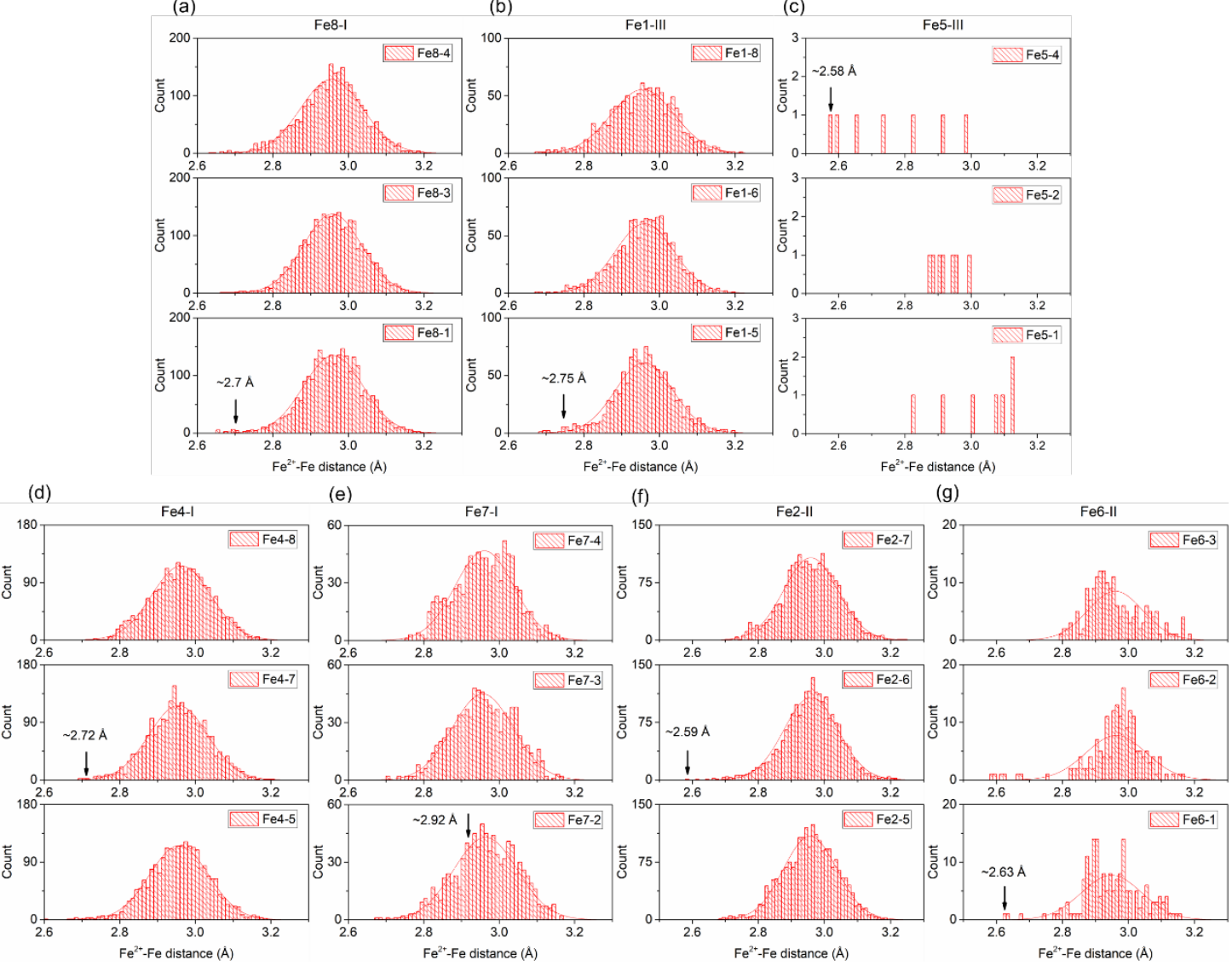

Figure S12. Probability distributions of the three neighboring $\mathrm{Fe}^{2+}$-Fe distances for the stages defined in Figure 2 in the last 100 ps of the AIMD simulation on e@EP Fe2O3: (a) Fe8-I, (b) Fe1-III, (c) Fe5-III, (d) Fe4-I, (e) Fe7-I, (f) Fe2-II, and (g) Fe6-II. The arrow indicates the $\mathrm{Fe}^{2+}$-Fe distance around which the EP hopping takes place. 

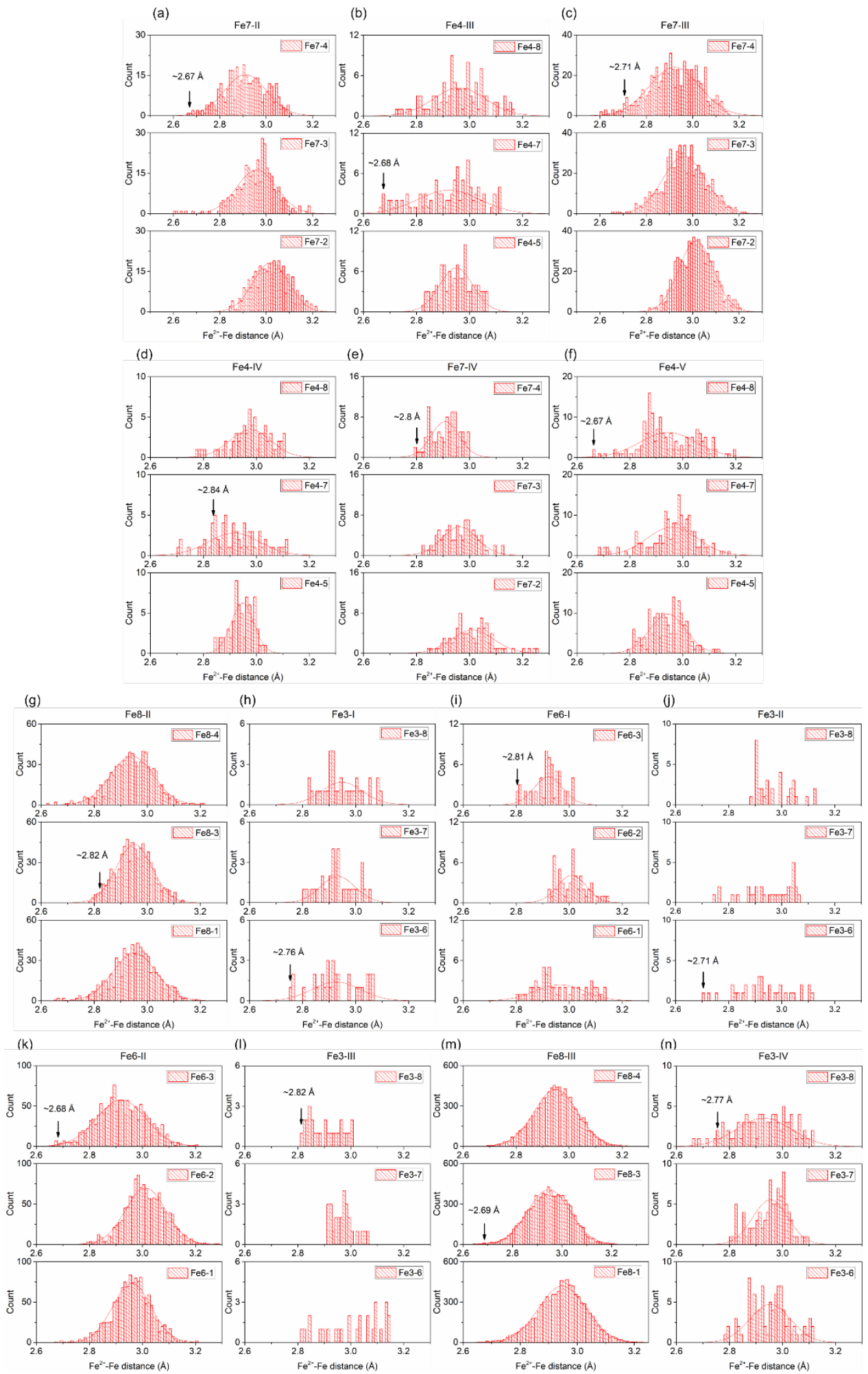

Figure S13. Probability distributions of the three neighboring $\mathrm{Fe}^{2+}$-Fe distances for the stages defined in Figure 6 in the last 130 ps of the AIMD simulation on $\mathrm{Si} @ E P \mathrm{Fe}_{2} \mathrm{O}_{3}$ : (a) Fe7-II, (b) Fe4-III, (c) Fe7-III, (d) Fe4-IV, (e) Fe7-IV, (f) Fe4-V, (g) Fe8-II, (h) Fe3I, (i) Fe6-I, (j) Fe3-II, (k) Fe6-II, (l) Fe3-III, (m) Fe8-III, and (n) Fe3-IV. The arrow indicates the $\mathrm{Fe}^{2+}$-Fe distance around which the EP hopping takes place. 


\section{The atomic coordinates (in the format of POSCAR as the input file of VASP) of}

geometries used for computing charge distributions and potential energy curves.

\section{The energies are given in parentheses.}

\section{(1) e@EP-Fe $\mathrm{F}_{2} \mathrm{O}_{3}$}

(1.1) $2 \times 2 \times 1$ supercell with the EP localized on Fe1 $(-823.203 \mathrm{eV})$

$\begin{array}{ccc}10.1413002014000000 & 0.0000000000000000 & 0.0000000000000000 \\ -5.0706501007000000 & 8.7826236017999992 & 0.0000000000000000 \\ 0.0000000000000000 & 0.0000000000000000 & 13.9027004242000007\end{array}$

$\mathrm{O} \quad \mathrm{Fe}$

$72 \quad 48$

0.1899439046058910

0.3335062666053893

$0.0164144372966085 \quad 0.0818431439837964$

0.4894133574279440

$0.3204762111418739 \quad 0.0831403660593800$

0.6806733155939675

$0.1677762272584277 \quad 0.0832779546275266$

0.8322708396580472

$0.0132583774239054 \quad 0.0834933847622376$

0.9850719987599056

0.3223620801729066

0.0830067663784279

0.1805217057316611

0.1764169380619916

0.0812548733262581

0.3350419047740289

0.5152155564982562

0.0830553308466757

0.4860096666799890

0.8202918576301451

0.0833021064994210

0.6793867674248375

0.6658700653526853

0.0833189522414184

0.8289129604530396

0.5124762032867517

0.0833595771507631

0.9871780416946722

0.8143949701540100

0.0831270662543499

0.1532052803850575

0.6674141612482662

0.0836771929910677

0.0003457513812870

0.1534126222283962

0.2504976012644073

0.3471297619500666

0.3472041644216941

0.2503408824636767

0.6533431473623231

0.9998139161487460

0.2505216150159085

0.4989682684090433

0.1529491068146111

0.2497661978784309

0.8424845486065276

0.3458831696537781

0.2497937954951331

0.0010639443819254

0.2548004962599445

0.1533711436752467

0.6525094178892676

0.2504271299141675

0.9982596957466023

0.8408421989367864

0.2542076564637967

0.3467569844364021

0.4999624197352083

0.2502973516182583

0.6525634279415974

0.6524973793542602

0.2506144188606859

0.4992249980477236

0.8463563650475691

0.2505639614755931

0.8467452436050867

0.4996438366950640

0.2501755374046456

0.0140251128347500

0.1798651222755045

0.4170194860830114

0.3201283763857782

0.3335526476962701

0.4171470846975747

0.1665080279751714

0.4862698040450954

0.4171483988624588

0.5134529293932533

0.1802825535200400

0.4167411803120052

0.8195124261423196

0.3328314323234672

0.4167507889144179

0.6666253165253250

0.4862187629947582

0.4167184112463326

0.0138246926629454

0.6786374338122494

0.4183135079018533

0.3201617152893043

0.8333972264382589

0.4173203088663170 


\begin{tabular}{|c|c|c|}
\hline & & \\
\hline 16 & 590 & 0. \\
\hline & 512 & \\
\hline 6 & 27 & \\
\hline & & \\
\hline & 485 & \\
\hline 21 & 523 & רחל \\
\hline & & \\
\hline & & \\
\hline 936 & 461 & \\
\hline & 42 & \\
\hline & & \\
\hline 455 & 251 & \\
\hline 348 & 0.81 & \\
\hline & & \\
\hline & & \\
\hline 71 & 377 & 0. \\
\hline 79 & 62 & \\
\hline & & \\
\hline 97 & 03 & \\
\hline 0.6 & 68 & \\
\hline & & \\
\hline & & \\
\hline & & \\
\hline 91 & 37 & \\
\hline & & \\
\hline & & \\
\hline & 04 & \\
\hline 0.32 & 0.487 & $0 . c$ \\
\hline 705 & 2 & \\
\hline & & \\
\hline $0.820 c$ & 0.487407 & 460 \\
\hline 53 & 24 & 680 \\
\hline & & \\
\hline 0.32 & & \\
\hline 0.16 & 0.68 & 807 \\
\hline & & \\
\hline & & \\
\hline 0.6665 & 12 & $015 \mathrm{c}$ \\
\hline 0.5 & 71 & 60970948 \\
\hline 7555744 & 374527715 & 0.02072 \\
\hline & & 0070 \\
\hline & & 0.0216191906162 \\
\hline 0.6667848892729212 & 0.8328906935925159 & 0.0211640369783529 \\
\hline
\end{tabular}




\begin{tabular}{|c|c|c|}
\hline 21 & 103 & 0.1 \\
\hline 0.5015469403439120 & 0.0015648457344164 & 0.1462580220739214 \\
\hline 9989125384674146 & 0.4993638286492303 & 0.1458951850421857 \\
\hline 02612496996373 & 0.4990923401850321 & 54771 \\
\hline 3296393506774641 & 0.1635437527471403 & 0.1862355809959459 \\
\hline 8363429389677370 & 0.1646091241413496 & 0.186782 \\
\hline 3332979123950129 & 0.6666285453020814 & 4936 \\
\hline 75 & 7032 & 0.18 \\
\hline 604456702757 & 0.3323791119825190 & 0.312 \\
\hline 0.6661234696168586 & 0.3325622754452890 & 0.3114261730363917 \\
\hline 45688286 & 70047339397 & 0.313 \\
\hline 0.6655225601440971 & 0.8327051726411848 & 0.31353 \\
\hline 0.9988618886370312 & 0.9983274188411809 & 0.3511795541458743 \\
\hline .5000243245984914 & 0.00015 & 0.3536977196878173 \\
\hline 222099 & 924570565 & 0.35 \\
\hline 0.499946501 & 0.4995 & 6230 \\
\hline 3328036760064061 & 0.1661963427032214 & 0.47906390 \\
\hline 8336110599246607 & 0.1662986008584370 & 0.478 \\
\hline 33 & & \\
\hline 27479 & 0.6665338162429650 & 0.479 \\
\hline 1664709916966347 & 0.3329852025382840 & 0.5202764719593340 \\
\hline .66658 & 0.333 & 0.52 \\
\hline .1664676282275734 & 0.83328258 & 0.52049 \\
\hline 0.6667680833855424 & 0.8333193864649220 & 0.5207755719704005 \\
\hline 0.0000823896281896 & 0.0000474482558939 & 0.6463686247693340 \\
\hline .5000490129921175 & 0.0000 & 0.6459 \\
\hline .0000059451875158 & 0.5000043611280276 & 0.6459305280430239 \\
\hline 0.4999004988774606 & 0.4998130128286007 & 0.6460866154942215 \\
\hline 0.3334209812031353 & 0.16666686662773835 & 0.6872406117289873 \\
\hline 8333228148688353 & 0.16677622260 & 0.68720 \\
\hline .3335234255336761 & 0.6667730176001405 & 0.6872642785275319 \\
\hline 0.8333012986912227 & 0.6666479700571163 & 0.6873018117962840 \\
\hline 1664769155029883 & 0.3335973071702046 & 0.8128233224490857 \\
\hline 6667515952728403 & 0.3334261959732956 & 0.8130299819078771 \\
\hline 0.1672599643001001 & 0.8337587417772716 & 0.8131768048770034 \\
\hline .6668375841415565 & 0.8331400329906561 & 0.8131436639701413 \\
\hline .0001005816397773 & 0.0001690966518672 & 0.8547518438564170 \\
\hline 0.5009609851375112 & 0.0002808728252048 & 0.8538660720961726 \\
\hline 0.0000946564654394 & 0.5010941102053000 & 0.8537210070089634 \\
\hline 0.4997887702634140 & 0.4996281355770961 & 0.8540654380728575 \\
\hline 0.3329526108323675 & 0.1654788041581841 & 0.9796964155352772 \\
\hline 0.8350473348986733 & 0.1687708302601669 & 0.9797166369556424 \\
\hline .3333799413853303 & 0.6670179255511901 & 0.9794946513017462 \\
\hline 8332533795472 & 0.667199312912584 & 0.980232508258121 \\
\hline
\end{tabular}


(1.2) $2 \times 2 \times 1$ supercell with the EP localized on Fe2 $(-823.203 \mathrm{eV})$

\begin{tabular}{llll}
0.1795459540506797 & 0.0129665938141586 & 0.0831790222721628 \\
0.3321153181831390 & 0.3216025984549323 & 0.0833486562930136 \\
0.4834716875592507 & 0.1735524744010640 & 0.0819449540527320 \\
0.6854201373848477 & 0.0141820823694550 & 0.0830970462940603 \\
0.8325818164907608 & 0.3196527594366551 & 0.0837464984032705 \\
0.9874100399591464 & 0.1668336263185195 & 0.0833964119551720 \\
0.1775765430235882 & 0.5098979110170390 & 0.0829636239041104 \\
0.3237695547201172 & 0.8086517825867995 & 0.0811095805008463 \\
0.4867376448858776 & 0.6672589440543325 & 0.0834891751355277 \\
0.6797459977364881 & 0.5146976524161410 & 0.0833885701762958 \\
0.8341522840550755 & 0.8200715546007350 & 0.0833498084851720 \\
0.9847107217314534 & 0.6652505858782020 & 0.0830506706209941 \\
0.1540616976584174 & 0.1531725023219622 & 0.2498656520687264 \\
0.0004118642956411 & 0.3471811677951376 & 0.2501936926791734 \\
0.3466907031999070 & 0.0000420778846190 & 0.2505399481833203 \\
0.6592696165390468 & 0.1576292405515571 & 0.2542182216582524 \\
0.5001916674716753 & 0.3474451736345539 & 0.2506011958074755 \\
0.8475749650504527 & 0.0001435995180412 & 0.2506576299600959 \\
0.1528026334796925 & 0.6531652406920102 & 0.2502975094231011 \\
0.0001021599751137 & 0.8468522996993331 & 0.2502812591879581 \\
0.3470969235823519 & 0.5004928256509426 & 0.2497808555347136 \\
0.6536631263070465 & 0.6529259640053922 & 0.2505615240701426 \\
0.4990851718105301 & 0.8415948921059462 & 0.2547596198014463 \\
0.8475586626499965 & 0.5009744032373390 & 0.2505075421561209 \\
0.0138268619313706 & 0.1805653074655468 & 0.4167258134709061 \\
0.3197584117194223 & 0.3333029984009279 & 0.4167841778702457 \\
0.1672062342904438 & 0.4868381980397496 & 0.4167363655971366 \\
0.5139358522091479 & 0.1803528273052988 & 0.4170715308581622 \\
0.8214103467846099 & 0.3352988137102147 & 0.4183417402173694 \\
0.6666253984090942 & 0.4868729017167203 & 0.4174102722703840 \\
0.0138481505970637 & 0.6804232672503577 & 0.4171477868386336 \\
0.3201930409357198 & 0.8342309701672367 & 0.4170242520049507 \\
0.1665958399955560 & 0.9867492515636177 & 0.4171699261525402 \\
0.5141952693717456 & 0.6791170022261646 & 0.4184388565548884 \\
0.8207547287478476 & 0.8337740104237673 & 0.4173812567722070 \\
0.6671389296835492 & 0.9863477235891326 & 0.4176928090703779 \\
0.486153422098689 & 0.3197557132496052 & 0.5834428414534781 \\
\hline
\end{tabular}




\begin{tabular}{cccc}
0.4863972222903146 & 0.8198580300797654 & 0.5836592035412962 \\
0.1803283273615479 & 0.6667540550376008 & 0.5832803762879978 \\
0.3332037158631643 & 0.5134995602170945 & 0.5834365292934011 \\
0.9867516783722008 & 0.8198951129171699 & 0.5834933604294871 \\
0.6805117746058658 & 0.6669317771556731 & 0.5837918033832358 \\
0.8331758188304553 & 0.5136497109392784 & 0.5836520879767192 \\
0.3466899077372969 & 0.3469777994198339 & 0.7495841557687578 \\
0.1531193561633790 & -0.0001811280061851 & 0.7497985344844430 \\
0.0001216700079002 & 0.1530775070865186 & 0.7497060750536104 \\
0.8463233326520493 & 0.3466485982872483 & 0.7498064712116660 \\
0.6531183592005487 & 0.0000927304994964 & 0.7500848810379900 \\
0.4998286494559788 & 0.1530036082860340 & 0.7500527537664599 \\
0.3468691445071756 & 0.8467729993353100 & 0.7498761310079923 \\
0.1527943399057053 & 0.4995217731014157 & 0.7494927007926626 \\
-0.0001951115196598 & 0.6528994116609382 & 0.7494365836134490 \\
0.8468218241724440 & 0.8469789605278698 & 0.7497653401864866 \\
0.6529321659797369 & 0.4999720453042049 & 0.7497219939051222 \\
0.5001306622294586 & 0.6534106374852645 & 0.7497871905805790 \\
0.3192627531355152 & 0.4862936721072069 & 0.9160194201454115 \\
0.1665453786794727 & 0.1800680945014932 & 0.9161757328498918 \\
0.0125059837987496 & 0.3324776876097823 & 0.9160493811726712 \\
0.8193789708009591 & 0.4864930972633833 & 0.9162737504729214 \\
0.6661452146012422 & 0.1800760327759093 & 0.9169432548986283 \\
0.5135739395050932 & 0.3342171707108295 & 0.9154691232272097 \\
0.3192797134583777 & 0.985979716063774 & 0.9163956184264263 \\
0.1652201109137609 & 0.6791290601475621 & 0.9148648611445135 \\
0.0126861458032332 & 0.8326402719479837 & 0.9160246831761033 \\
0.8199566008332647 & 0.9863792791245740 & 0.9160229105373938 \\
0.6667892981433199 & 0.6802812085513964 & 0.9163170223594652 \\
0.5133507587661924 & 0.8330885616165559 & 0.9166549902069697 \\
0.1660942620386500 & 0.3326984939300468 & 0.0205441250667667 \\
0.6650518278781912 & 0.3330454977798866 & 0.0217039623632539 \\
0.1658699848146725 & 0.8324814531513585 & 0.0206976862119048 \\
0.6671097402037679 & 0.8337157761297799 & 0.0211085449465001 \\
0.0008755755427146 & 0.0010653558802755 & 0.1461529655901155 \\
0.5005540735135838 & -0.0036031463619514 & 0.1448247039150745 \\
0.0007219307098159 & 0.4995958026303235 & 0.1459104003611944 \\
0.4984181113422448 & 0.4999982886903614 & 0.1463020708114723 \\
0.3363742628222374 & 0.1662830600020117 & 0.1863267778701261 \\
\hline
\end{tabular}




$\begin{array}{ccc}0.1676907641375556 & 0.8341610754717386 & 0.3125378032201558 \\ 0.6674226324055872 & 0.8329354237708556 & 0.3135485762535850 \\ 0.0005283535362573 & 0.0005173860075840 & 0.3536002776104014 \\ 0.5017273748288946 & 0.0006331003606054 & 0.3512054233968023 \\ 0.0005538932739447 & 0.5004611006264749 & 0.3537616597386800 \\ 0.4998636536607104 & 0.4999993035584500 & 0.3537403191476711 \\ 0.3339071193874972 & 0.1667376412999608 & 0.4790641443073122 \\ 0.8335195635134279 & 0.1669442741986872 & 0.4792624140300683 \\ 0.3337219615492010 & 0.6674390368612860 & 0.4789527078791336 \\ 0.8336374709318061 & 0.6668313638762535 & 0.4795136454046224 \\ 0.1668696174104475 & 0.3335107555233279 & 0.5201401692763616 \\ 0.6667375733523584 & 0.3332309532080662 & 0.5205588133273219 \\ 0.1670488665119504 & 0.8336144305977792 & 0.5202877657366693 \\ 0.6667204458306477 & 0.8335464201791151 & 0.5208252780588257 \\ 0.0002049774090893 & 0.0000908607873348 & 0.6460618927961130 \\ 0.4999587475432061 & 0.0000566811555104 & 0.6464030844838820 \\ 0.0000091278970430 & 0.5000317609270919 & 0.6459512244933737 \\ 0.4999400021177360 & 0.5000092302333726 & 0.6460209630518562 \\ 0.3332674964218123 & 0.1666514006671312 & 0.6872711174337968 \\ 0.8333467555165482 & 0.1666528414238038 & 0.6873543700021463 \\ 0.3331843587910526 & 0.6664726496113830 & 0.6871968001795384 \\ 0.8331787105303768 & 0.6666649849080745 & 0.6872976024866878 \\ 0.1665491720122536 & 0.3332018396829562 & 0.8130061953214023 \\ 0.6661394027264063 & 0.3333447094502760 & 0.8132609175107350 \\ 0.1663743227126957 & 0.8327242674290043 & 0.8128336435467763 \\ 0.6668507150567854 & 0.8336147287331493 & 0.8131251548930170 \\ 0.0002805214219926 & 0.0000303330695172 & 0.8540702756911482 \\ 0.4997283013068499 & -0.0002285779590672 & 0.8547875846108931 \\ -0.0011674585727548 & 0.4988287855871795 & 0.8537546980446024 \\ 0.4996949874951968 & 0.5005291350935406 & 0.8539224766071756 \\ 0.3343891854077856 & 0.1673442607798324 & 0.9797584585738305 \\ 0.8327092856055195 & 0.1658960705503782 & 0.9802864359554816 \\ 0.3312258628783504 & 0.6661871462521930 & 0.9796142389171832 \\ 0.8329666899974602 & 0.6662315798785281 & 0.9795597475665576\end{array}$

(1.3) $2 \times 2 \times 1$ supercell with the EP localized on Fe3 $(-823.203 \mathrm{eV})$
0.1797468872428310
$0.0147398649783258 \quad 0.0833205818239122$
0.3342276003595253
$0.3201029231404598 \quad 0.0832817841708414$
0.4847644355574410
0.1652438615250425
0.0830174258409209
0.6776172782235478
0.0099356097805923
0.0829742697460311
0.8237305112149869
$0.3086698922211249 \quad 0.0811685512609788$
0.9867471871787996
$\begin{array}{ll}0.1673537536770548 & 0.0834447188837810\end{array}$
0.1855025635868567
$\begin{array}{ll}0.5143182228928651 & 0.0830690299539281\end{array}$
0.3326338075920828
$\begin{array}{lll}0.8196848493033481 & 0.0836796800488668\end{array}$ 


\begin{tabular}{|c|c|c|}
\hline & & \\
\hline & 21 & \\
\hline 25046750 & 8216500946059963 & \\
\hline 034571038 & & \\
\hline & & \\
\hline & 922 & \\
\hline & & \\
\hline & & \\
\hline & & \\
\hline 547 & 0.000 & \\
\hline & & \\
\hline & & \\
\hline 70 & 07 & \\
\hline 38 & & \\
\hline & & \\
\hline 0. & 4 & \\
\hline 417 & 0.17 & \\
\hline 01 & 30 & \\
\hline & & \\
\hline 31 & & \\
\hline 885 & 13 & \\
\hline & & \\
\hline & & \\
\hline 0.32 & 98 & \\
\hline 0.1 & 0.9 & \\
\hline 0.513 & & \\
\hline & & \\
\hline 915 & & \\
\hline 0.4866 & & \\
\hline & & \\
\hline & & \\
\hline 0.98 & 72 & \\
\hline & & \\
\hline & & \\
\hline 0.4866692225665622 & 0.8197240465186436 & 58346342396 \\
\hline 9450541 & 602 & 0.5 \\
\hline & & \\
\hline 0.9864019154334530 & 0107657751005010 & 05024062412742 \\
\hline 0.6 & 17 & 779 \\
\hline & & \\
\hline & 85725 & 07407451276415 \\
\hline 0.1529919398035921 & 0.0000045240298624 & 0.7496824627338 \\
\hline 0.00012235568417 & 0.153 & 0. \\
\hline 040 & 400 & $0.1490 / 00500009$ \\
\hline
\end{tabular}




\begin{tabular}{|c|c|c|}
\hline \multicolumn{2}{|c|}{$0.6528412897712472-0.0004123007508485$} & \\
\hline 772 & 240197 & \\
\hline & 8466473836805204 & \\
\hline & & \\
\hline & & \\
\hline 0.84 & 584 & \\
\hline & & \\
\hline & & \\
\hline & & \\
\hline 5 & 6730 & \\
\hline & & \\
\hline & & \\
\hline 315 & 376 & \\
\hline 0.51 & 86 & \\
\hline & & \\
\hline 0.1 & 03 & \\
\hline 0.01 & 409 & \\
\hline 0.81 & 347 & \\
\hline & & \\
\hline 0.5 & 267 & \\
\hline 346 & 724 & \\
\hline 0.6 & & \\
\hline & & \\
\hline 0.66 & 79 & \\
\hline-0.00 & 0.000 & 0.1 \\
\hline 0 & & \\
\hline 0.0 & & \\
\hline 0.50 & 721 & \\
\hline 0.33 & 54 & \\
\hline & & \\
\hline 0.33 & 0.6 & \\
\hline 0.83 & & \\
\hline & & \\
\hline & & \\
\hline 0.1673 & 0.8342 & 0.3131 \\
\hline 0.6674 & 0.8336672015109533 & 0.3 \\
\hline & & 0.35 \\
\hline 0.50 & 0.0004291147633346 & 2527405051050 \\
\hline 31 & 62 & 0.3 \\
\hline & 41 & \\
\hline & 081434 & 0.4794 \\
\hline 0.8336708043362374 & 0.1673772686125502 & 0.4789407495094 \\
\hline & & 0. \\
\hline 033 & 000114950454 & $0.4 / 90 / 18818130$ \\
\hline
\end{tabular}




\begin{tabular}{|c|c|c|}
\hline & 72 & \\
\hline 527 & 50071000 & 0. \\
\hline 1666879931343134 & 8331900042780312 & \\
\hline . & & \\
\hline 267148711 & 34 & 0.645 \\
\hline 0.50 & 60 & 0.64 \\
\hline-0.00 & 48 & \\
\hline & & \\
\hline 0.3 & & \\
\hline 36447 & 620 & \\
\hline 8 & 66 & \\
\hline & & \\
\hline 883 & 532 & 799 \\
\hline 0.66 & 0.33 & \\
\hline 0.1 & 0.8 & \\
\hline 0.6 & 15 & \\
\hline-0.0002 & 0.00 & \\
\hline 0.4989 & -0.00 & 0.8 \\
\hline-0.0 & & \\
\hline 0.50 & 672 & 0.8 \\
\hline 0.33 & 289 & \\
\hline 0.83 & 36 & \\
\hline & & \\
\hline & & \\
\hline \multicolumn{3}{|l|}{ 1.4) $2 \times 2 \times 1$ supercell } \\
\hline & & \\
\hline 54 & 368 & \\
\hline 72 & 08 & 438 \\
\hline & 82 & \\
\hline & & \\
\hline 58 & 64 & 163 \\
\hline 27 & 00 & 514 \\
\hline & & \\
\hline 7361 & 06735489851 & \\
\hline 0.6853848919500241 & 765727362 & 232782 \\
\hline & & \\
\hline & & \\
\hline 0.1528792971052447 & 0.1532647710212051 & 0.2502 \\
\hline & 0.346 & \\
\hline 391 & 0.000558766 & 0.2497244969112869 \\
\hline & 65925935 & 0.25 \\
\hline & 0.341596 & $0.254800182412 €$ \\
\hline 0.8476218552150809 & 0.0010533605042919 & 0.2505017298924438 \\
\hline
\end{tabular}




\begin{tabular}{|c|c|c|}
\hline 1541145589922263 & 50 & \\
\hline 0.0004659 & .8472443708833877 & 0.25015530 \\
\hline 3467433995666365 & .5000738319695621 & .2505474682227289 \\
\hline 7 & 13 & 475846 \\
\hline 964 & 52 & 2809 \\
\hline 41 & 0.5 & 094 \\
\hline 65 & 02 & \\
\hline & & \\
\hline 19 & 0.4 & 024 \\
\hline 5142729 & 0.17919304 & 1897 \\
\hline 2 & 0. & \\
\hline 69 & 0.48 & 3291 \\
\hline 0.01 & 0.68 & 994 \\
\hline 32 & 0.83 & \\
\hline & & \\
\hline 0.5 & 0.6 & 07 \\
\hline 92164 & 0.8354 & 986 \\
\hline 87 & 0.986 & 193 \\
\hline & & \\
\hline 713 & 0.1 & 684 \\
\hline 0389 & 0.0135332 & 0.58 \\
\hline 3 & 0.3 & \\
\hline 57 & 0.16 & \\
\hline 0.8332267 & 913919 & 3857 \\
\hline 0.486427 & 909408917 & 129 \\
\hline 4 & 0.6 & 48 \\
\hline 82 & 0.51 & 921 \\
\hline 337392995433 & 0.8198119721 & 24116 \\
\hline 0.6802673525505322 & 0.6667705475794102 & 2847549 \\
\hline & & \\
\hline 5228 & 0.3467060861725801 & 0.7498225 \\
\hline 0.152 & 000 & 0.749 \\
\hline 0002500/ J5004052 & 1528139315218612 & 0.749398 \\
\hline 8467706731534216 & 0.3469128887600286 & 0.7497 \\
\hline 0.65289425674792 & -0.0000974879837472 & 0.7497082578780206 \\
\hline 5000594209397866 & 0.1533265034044905 & 0.7497605181405430 \\
\hline 647 & 067308033401 & $0.74 \mathrm{~S}$ \\
\hline 1530683119156481 & 0.4997688924859817 & 0.7497495016539822 \\
\hline 0.0000560544531341 & 0.6529826717742016 & 0.7497121288580944 \\
\hline 079 & 0080564854 & 0.7498078 \\
\hline 0772955800839 & 0.5000119901309723 & 0.7500601033695350 \\
\hline 0.4997845323492358 & 0.6529354100369910 & 0.7500398624858584 \\
\hline 319211 & 0.4859021570113 & 0.91636502523 \\
\hline 21050320 & $1 / 90050094003$ & 0.914010494505 \\
\hline
\end{tabular}




\begin{tabular}{|c|c|c|}
\hline 470 & 0.33 & $0 . \subseteq$ \\
\hline 0.8198782709104875 & 0.4862877048420708 & 0.9160188556483588 \\
\hline 6667076596266865 & 0.1801814417126341 & 0.9162857452775538 \\
\hline 2926104252787 & 0.3330014424202091 & 0.9166008889016891 \\
\hline 3191919445257074 & 0.9862069689406185 & 0.9159683919218028 \\
\hline 1664602115938064 & 66929977154 & 9887770 \\
\hline 0124056449156965 & 0.8323422885269557 & 0.91 \\
\hline 6877 & 633455343671 & 396 \\
\hline 9802785088 & 0.6799535528698977 & 398175 \\
\hline 0.5135116690824880 & 0.8341256034681285 & 0.9154734765860518 \\
\hline 8613387780607 & 0.3324253209601774 & 735755 \\
\hline 0.6671008119136995 & 0.3336931574921195 & 9890381484 \\
\hline 0.1660011491376315 & 0.8326176500164478 & 0.0205097397101421 \\
\hline 0.6650121435347204 & 0.8329662476581986 & 0.0217139638821349 \\
\hline 7255344138573 & -0.0003982207279100 & 885292212673 \\
\hline 1758 & 0.00 & 655 \\
\hline 0.0008965475889059 & 0.5010443652462268 & 0.1461210185183964 \\
\hline 5004678184311419 & 0.4963628397954032 & 0.1448 \\
\hline 14 & 0.1 & \\
\hline 0.8334016379932808 & 0.1666736371503254 & 0.1871765450643681 \\
\hline .3364129753582219 & 0.6663314985143760 & 0.1863168929820630 \\
\hline ).8311514842868845 & 0.66 & 0.188043490941 \\
\hline .1677468494337253 & 0.334237640652 & 0.312498 \\
\hline 0.6675116787348937 & 0.3329740891353585 & 0.3135659577516050 \\
\hline .1675166167168471 & 0.8338068924952748 & 0.3114477422137347 \\
\hline .6674292569975158 & 0.8343113565 & 0.3131796 \\
\hline .0006518230294153 & 0.0005922125459739 & 0.3537081125819618 \\
\hline 0.4999530551587240 & 0.0000930291573783 & 0.3537086167300447 \\
\hline 0.0006275410552356 & 0.5006528064440082 & 0.3535606797177064 \\
\hline 587 & 0.5007339325229684 & 0.3512350424245297 \\
\hline .3337931276624126 & 0.1675142623408463 & 0.4789247879906160 \\
\hline 0.8337104418571557 & 0.1669269940088066 & 0.4794718391529229 \\
\hline 3339570214894712 & 0.6668478707743578 & 0.4790513362604233 \\
\hline 8336153708549686 & 0.6670775601737631 & 0.4792613949473643 \\
\hline 0.1671159726086883 & 0.3336894504866775 & 0.5202500778434559 \\
\hline .6667875980951681 & 0.3336323032634859 & 0.5208337791220042 \\
\hline .1668947176160828 & 0.8335670420349146 & 0.5200942366772657 \\
\hline 0.6667714498586370 & 0.8333032266435788 & 0.5205800475749099 \\
\hline 0.0000097631618688 & 0.0000422257116815 & 0.6459223868329012 \\
\hline 0.4999390031365915 & 0.0000263768424713 & 0.6459973324358805 \\
\hline 0.0002308008321663 & 0.5001029500990144 & 0.6460280147125723 \\
\hline 0.4999693463304537 & 0.5000645874411851 & 0.6463751552508293 \\
\hline .3331355202075558 & 0.1664159313585710 & 0.6871669689467332 \\
\hline 83310654352513 & 0.166607434304305 & 0.687273206878647 \\
\hline
\end{tabular}




$\begin{array}{cll}0.3332458045117614 & 0.6666181302590317 & 0.6872572126401315 \\ 0.8333110929339359 & 0.6666025555827786 & 0.6873615556322887 \\ 0.16627745744911925 & 0.3326399350664311 & 0.8127695584683217 \\ 0.6667842309186296 & 0.3335152724278619 & 0.8130914676261249 \\ 0.1664865289154772 & 0.8331218193225558 & 0.8129802157728003 \\ 0.6660807384389682 & 0.8332598137252860 & 0.8132739493633726 \\ -0.0012399719726091 & -0.0012762328088864 & 0.8537335480056757 \\ 0.4996265554645943 & 0.0004122072708792 & 0.8538943488150317 \\ 0.0001889714992081 & 0.4999111558555656 & 0.8540467979969809 \\ 0.4996436641813404 & 0.4996675323490507 & 0.8547484041180669 \\ 0.3311683005899825 & 0.1660942928734377 & 0.9795771768475786 \\ 0.8328920031905034 & 0.1661190061694215 & 0.9795323169220234 \\ 0.3343067467317206 & 0.6672475136796856 & 0.9797520078929414 \\ 0.8325931793074005 & 0.6657843976323746 & 0.9802951296436833\end{array}$

(1.5) $2 \times 2 \times 1$ supercell with the EP localized on Fe5 (-823.203 eV)

$\begin{array}{clc}0.1799716661305856 & 0.0132731111377156 & 0.0828007687726189 \\ 0.3348785804450521 & 0.3258438193510401 & 0.0791522773875764 \\ 0.4908750404109400 & 0.1657108688485535 & 0.0785785417464477 \\ 0.6798654746762419 & 0.0137569355045823 & 0.0835706634827674 \\ 0.8340925781555830 & 0.3203341311861355 & 0.0827972069254225 \\ 0.9860631298278936 & 0.1668069193950552 & 0.0827181721092033 \\ 0.1799037743070672 & 0.5142545913814115 & 0.0828310700038620 \\ 0.3329148914750120 & 0.8194858989470534 & 0.0830659800426808 \\ 0.4865540508182298 & 0.6670752623653851 & 0.0831817114571998 \\ 0.6807386724272932 & 0.5141657840835591 & 0.0826931954219957 \\ 0.8342357760625007 & 0.8207508680270541 & 0.0835192506266905 \\ 0.9865383202470295 & 0.6666975918199471 & 0.0830326809532644 \\ 0.1432591708985081 & 0.1502446403420433 & 0.2513805447233722 \\ -0.0016442875334252 & 0.3463760950945879 & 0.2499777153853667 \\ 0.3483898339192439 & 0.9904120820698674 & 0.2522251570021672 \\ 0.6527333484167462 & 0.1533794239577062 & 0.2499279091155206 \\ 0.5045696585602112 & 0.3521745736487378 & 0.2502829217194559 \\ 0.8438322841670721 & -0.0012365715835610 & 0.2499602104730684 \\ 0.1528845123072632 & 0.6514149691018909 & 0.2503376176109005 \\ -0.0000853108248060 & 0.8462764027537802 & 0.2501830334032661 \\ 0.3462809062208012 & 0.4992662634922903 & 0.2496059927118702 \\ 0.6539694517055246 & 0.6541186089783964 & 0.2499451085041613 \\ 0.5009609315930168 & 0.8442796775033903 & 0.2504149467485430 \\ 0.8474667060231700 & 0.5008831740817583 & 0.2500435516376472 \\ 0.0127160574256171 & 0.1802354537721678 & 0.4178519687498740 \\ 0.3193517901802521 & 0.3328186515155689 & 0.4164130436567192 \\ 0.1662801920903808 & 0.4861056378742415 & 0.4170942742756387 \\ 0.5136819829412396 & 0.1801151535539677 & 0.4167445416943552\end{array}$




\begin{tabular}{|c|c|c|}
\hline & & \\
\hline & 6432 & 0 . \\
\hline 0133729991538609 & 6793912493263425 & \\
\hline 6 & & ( \\
\hline & & 25 \\
\hline & 273 & \\
\hline & & \\
\hline & & \\
\hline & & \\
\hline 84 & 0 & 0 . \\
\hline & & \\
\hline & & \\
\hline 0.68003 & 0.16 & 0.5 \\
\hline 0.83 & 0.01 & \\
\hline & & \\
\hline 77 & 0. & \\
\hline 616 & 0.5 & 596 \\
\hline 42 & 0.8 & 0 . \\
\hline & & \\
\hline & & \\
\hline & 0.3 & \\
\hline 5 & & \\
\hline & & \\
\hline 01 & 0.34 & \\
\hline$c \square$ & -0.0 & 0.74 \\
\hline 4 & & \\
\hline 0 & & \\
\hline 35 & 0.49 & 0.7 \\
\hline 2 & 0. & 81 \\
\hline & & \\
\hline & 0.50 & \\
\hline 500 & 0.6 & \\
\hline & & \\
\hline & & \\
\hline 0.01305 & 0.33325730300 & 0.9159505463420894 \\
\hline 4000 & 960360844 & 0.91 \\
\hline & & \\
\hline 0.5140933706588912 & 0.3337346324280719 & 0.9156451349748240 \\
\hline 929 & 0.986 & 0.91 \\
\hline & 0.6 & 0.9 \\
\hline 904679611 & 0.83319 & 0.91620 \\
\hline 0.8197337434504984 & 0.9863265531647800 & 0.9165822150909341 \\
\hline & 0.6 & 0.9 \\
\hline 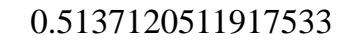 & S & J \\
\hline
\end{tabular}




\begin{tabular}{|c|c|c|}
\hline & 3 & \\
\hline 01 & 82652 & \\
\hline 1667965615739640 & 8343138600526803 & \\
\hline & & \\
\hline & & \\
\hline 0 & 28 & \\
\hline 26 & & \\
\hline & & \\
\hline & & \\
\hline 26 & 0.16 & \\
\hline & & \\
\hline & & \\
\hline 0.1 & 0. & 704 \\
\hline 77 & 0. & \\
\hline & & \\
\hline 0.6 & 0. & \\
\hline 0.0003 & 0.00 & \\
\hline 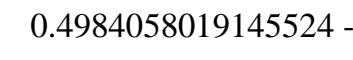 & & 0. \\
\hline & & \\
\hline 0.50 & 0.4 & \\
\hline 270 & 0.16 & \\
\hline 0.83 & & \\
\hline & & \\
\hline 0.83 & 0.6 & \\
\hline 63 & 0.33 & 0.52 \\
\hline 0.66 & 0.3 & \\
\hline & & \\
\hline 0.66 & 0.83 & 340 \\
\hline .000 & 0.00000500250 & 0.64 \\
\hline & & \\
\hline 37 & 0.49 & 0.646 \\
\hline 0.500 & 0.5 & \\
\hline & & \\
\hline & & \\
\hline 71729540 & 0.6666948625072345 & 0.6874 \\
\hline 19 & 0.666873 & 0.687 \\
\hline & & \\
\hline 0.6665161710476593 & 0.33334438884990 & 0.8125511740035878 \\
\hline 59 & 0.83 & 0.81 \\
\hline & 0.8 & 851 \\
\hline & 0.00048 & 0.8542760377610 \\
\hline 0.4995908856540410 & 0.00035623529177 & 0.8544070290477866 \\
\hline 2 & 0.5 & 0.85 \\
\hline $49900034 / 0000$ & 07012 & $0.85409224 / 9964$ \\
\hline
\end{tabular}




$\begin{array}{lll}0.3344157624586796 & 0.1683984975322698 & 0.9821415199436866 \\ 0.8331787777290417 & 0.1664480878433171 & 0.9796003864952514 \\ 0.3334537808214362 & 0.6672808151648340 & 0.9795782650318268 \\ 0.8332786451130790 & 0.6671672662016915 & 0.9797350256407779\end{array}$

(1.6) $2 \times 2 \times 1$ supercell with the EP localized on Fe6 $(-823.203 \mathrm{eV})$

\begin{tabular}{llll}
0.1804407828442546 & 0.0133522623623303 & 0.0831143203578553 \\
0.3342048626050084 & 0.3200087691849950 & 0.0828873750180616 \\
0.4863852277628883 & 0.1668918225099215 & 0.0829895585772178 \\
0.6747472252833300 & 0.0090062751081795 & 0.0785492251450648 \\
0.8331953431792603 & 0.3198782707365364 & 0.0827341615199913 \\
0.9907753709444266 & 0.1647653407171966 & 0.0789816180791834 \\
0.1806552005814822 & 0.5137339272648594 & 0.0828163743061207 \\
0.3336461938213983 & 0.8199005941116796 & 0.0835758617331391 \\
0.4861803400504136 & 0.6658148846917428 & 0.0827199197711657 \\
0.6801167087585501 & 0.5133233515748712 & 0.0830550464416460 \\
0.8333623721572981 & 0.8191207797807502 & 0.0826449395352771 \\
0.9864377188476053 & 0.6656952491018128 & 0.0835710150597075 \\
0.1529950461474759 & 0.1538763312726815 & 0.2495847588069625 \\
0.0068015911855264 & 0.3566298084027149 & 0.2514862354857684 \\
0.3432102318456908 & 0.9989824392289041 & 0.2503141364964631 \\
0.6420077705902828 & 0.1517768186050256 & 0.2520627076577684 \\
0.4986137420416094 & 0.3472586277667452 & 0.2502749151650529 \\
0.8478425114186953 & 0.9955720647405645 & 0.2502064305302962 \\
0.1549865023138574 & 0.6560763065920642 & 0.2500924139428209 \\
0.0002320891976211 & 0.8460862230771824 & 0.2499834428376452 \\
0.3481275766810896 & 0.5017043224338308 & 0.2500181772041155 \\
0.6534276936471917 & 0.6527639339154313 & 0.2499808061761012 \\
0.5005884122948839 & 0.8473011806980340 & 0.2498133975994773 \\
0.8463400359713393 & 0.5002092415504987 & 0.2502034619665975 \\
0.0136378615614119 & 0.1807974884573969 & 0.4164755344017925 \\
0.3200682296463488 & 0.3340716275961466 & 0.4171480557822184 \\
0.1676131011647791 & 0.4874043209725382 & 0.4179052686379391 \\
0.5125896415507935 & 0.1807674813421452 & 0.4183867720268175 \\
0.8193588778205523 & 0.3334692853399375 & 0.4169822527695462 \\
0.6661437913374871 & 0.4868714465648230 & 0.4172993604205121 \\
0.0135538941760092 & 0.6803240742453753 & 0.4171963081104861 \\
0.3197831269004441 & 0.8338575839557455 & 0.4172905323944537 \\
0.1660248630125574 & 0.9868602753815644 & 0.4172631777011280 \\
0.5137412572624621 & 0.6804643476962946 & 0.4169566401969628 \\
0.8198449802882597 & 0.8333166765469617 & 0.4173044790662364 \\
0.48638618879787979 & 0.3198963592080345 & 0.5839343221735982 \\
\hline
\end{tabular}




\begin{tabular}{|c|c|c|}
\hline 6 & 0.0135829028 & \\
\hline 0.9 & 0.3200030791159456 & 48 \\
\hline 6802381547076849 & .1667561296435776 & .5834730876871069 \\
\hline 9478 & 21 & 63 \\
\hline 9 & 27 & 24580 \\
\hline 6 & 89 & 602 \\
\hline & & \\
\hline & & \\
\hline 0.68 & & 179 \\
\hline 0.833 & 32511 & 0.58 \\
\hline & & \\
\hline & & \\
\hline 838 & 548812435 & 0.749 \\
\hline 0.84 & 33501 & 5382 \\
\hline & & \\
\hline 32 & 0. & 217 \\
\hline 5225 & 0.84688 & 3130 \\
\hline 51 & 0.4 & 0. \\
\hline & & \\
\hline 749 & 7757 & 2744 \\
\hline 16090 & 0.4 & 0.7 \\
\hline 0 & & \\
\hline 33 & & \\
\hline 056 & 0.18 & 0.9 \\
\hline 10 & 0.33 & 0.9 \\
\hline 34 & 0 & \\
\hline 38 & 0.1 & \\
\hline 8150 & 0.33 & 30663 \\
\hline $32000<0<>-1$ & 0.9 & 801 \\
\hline & & \\
\hline 31 & 0.83 & 0.91 \\
\hline 0.81944 & 0.98 & 04125 \\
\hline & 0.67 & \\
\hline & & \\
\hline 0.1674747727971692 & 0.3333909039729578 & 0.0201854279025579 \\
\hline 6673337435462730 & 0.3329859028619471 & 0.0207755679441405 \\
\hline 9390 & 0.83 & 0.02184 \\
\hline 0.6661556067724514 & 0.832086720663625 & 0.0197275896212297 \\
\hline 304907991 & 0.00127909 & 0.1452509 \\
\hline 02 & 0.0028 & 0.14654670 \\
\hline 9994227718032853 & 0.4963590692792 & 0.1470712725047292 \\
\hline 0.4999763303046399 & 0.4999104554417445 & 0.1461310577615328 \\
\hline 5 & 0.165 & 4519079 \\
\hline 02904530010 & .1024005010014 & 0.100 \\
\hline
\end{tabular}




$\begin{array}{ccc}0.3332402372367046 & 0.6681403785698216 & 0.1870570017736732 \\ 0.8344256096287006 & 0.6669308710294433 & 0.1872246004446616 \\ 0.1663959213278817 & 0.3347919419052556 & 0.3117081797134462 \\ 0.6658317987450658 & 0.3333805669823395 & 0.3126238638114920 \\ 0.1660883209930923 & 0.8332831850782801 & 0.3128083784906299 \\ 0.6671448360023429 & 0.8335554052288819 & 0.3121561207160968 \\ -0.0005525784300095 & 0.0000585505355668 & 0.3530343954817633 \\ 0.4996124845296180 & 0.0018359839293210 & 0.3536207502191261 \\ 0.0007262722911252 & 0.4996617425853005 & 0.3536351149423480 \\ 0.4997389219553616 & 0.5002098569341717 & 0.3537933615792886 \\ 0.3323681964624267 & 0.1669257292196325 & 0.4796071388622547 \\ 0.8333083123958518 & 0.1669157616923523 & 0.4785848423826445 \\ 0.3340082889503248 & 0.6677153490842994 & 0.4794055364998306 \\ 0.8335166856304294 & 0.6666546589357855 & 0.4792410771200332 \\ 0.1668711712056863 & 0.3340592464445762 & 0.5201403162280388 \\ 0.6662362603711184 & 0.3332620438684910 & 0.5205141942785891 \\ 0.1666822485426870 & 0.8335264058143285 & 0.5203260814497481 \\ 0.6670756082525511 & 0.8336432204680030 & 0.5201494222575461 \\ 0.0000699892331383 & 0.0000673307485518 & 0.6459784614829686 \\ 0.4999089252832142 & 0.0000569948198539 & 0.6460863521053075 \\ 0.0001179137298592 & 0.5001748913306878 & 0.6460837257631109 \\ 0.5001549630632632 & 0.5002867026139185 & 0.6460440776655706 \\ 0.3333537305503037 & 0.1666665451101264 & 0.6874294024597780 \\ 0.8333931003150384 & 0.1667649621460433 & 0.6869450623646614 \\ 0.3333474275157031 & 0.6667076041090803 & 0.6873037624730673 \\ 0.8334415963621784 & 0.6665334533672665 & 0.6872349872752666 \\ 0.1664936876126110 & 0.3330162281542234 & 0.8127858470753789 \\ 0.6668136020311952 & 0.3330470125677299 & 0.8130340514778405 \\ 0.1668025992948184 & 0.8331754644785524 & 0.8131933935050444 \\ 0.6668204109315891 & 0.8333545689706664 & 0.8124569619967754 \\ 0.0001173379679364 & -0.0001610668558745 & 0.8540625290332787 \\ 0.5006294969445765 & 0.0001137035054232 & 0.8543505237177804 \\ 0.9999306061360429 & 0.4993501757404679 & 0.8542687076579987 \\ 0.5000177948787228 & 0.4996586814418665 & 0.8538494464986023 \\ 0.3336455412132658 & 0.1663298148770595 & 0.9795801010946448 \\ 0.8338629681001152 & 0.1654146900496500 & 0.9820655554375648 \\ 0.8337104419730185 & 0.6664561292963627 & 0.9797624173330560\end{array}$

(1.7) $2 \times 2 \times 1$ supercell with the EP localized on Fe7 (-823.203 eV)
0.1799403023832465
0.0141772161608395
0.0828851101325419
0.3329720495410334
$0.3194192053115265 \quad 0.0830000634056613$
0.4866188839717062
$\begin{array}{ll}0.1670609605730682 & 0.0831296739675517\end{array}$
0.6807658138782259
$0.0141804788422706 \quad 0.0827128093930937$ 


\begin{tabular}{|c|c|c|}
\hline & & \\
\hline 0. & 0772 & \\
\hline 1763231009180 & 5132982329729111 & \\
\hline & 62 & \\
\hline 50 & 308 & \\
\hline & 04 & \\
\hline 727 & & \\
\hline & & \\
\hline & & \\
\hline 719 & 0.34 & 90 \\
\hline & & \\
\hline & & \\
\hline 0.5 & 880 & \\
\hline 0.8 & 0.0 & \\
\hline & & \\
\hline-0.0 & 0. & \\
\hline 309 & $0.4 \varsigma$ & \\
\hline 68 & & \\
\hline & & \\
\hline 10 & & \\
\hline 772 & 41 & \\
\hline & & \\
\hline & & \\
\hline 39 & & \\
\hline 0.81 & 0. & \\
\hline 2 & 6 & \\
\hline & & \\
\hline 30 & 293 & \\
\hline 0.16 & & \\
\hline & & \\
\hline & & \\
\hline 0.66 & 97 & \\
\hline & & \\
\hline & & \\
\hline 0.3329927772527496 & 0.0130433025948 & 0.583 \\
\hline 6482 & 319 & 901 \\
\hline & & \\
\hline 0.833 & 0,013 & 058 \\
\hline $0.4 \varepsilon$ & 22 & 295 \\
\hline & & \\
\hline & 666 & \\
\hline 0.9862301699163799 & 0.8196391243204890 & \\
\hline & 33 & 0. \\
\hline 83298206329028 & 15 & 0.50 \\
\hline
\end{tabular}




\begin{tabular}{|c|c|c|}
\hline 55 & 78 & 0 \\
\hline 1657904190 & 3256937780 & 0.7497164125699416 \\
\hline 0001704823343600 & 0.1533858110273123 & 0.7498231848328143 \\
\hline 8468606773170443 & 0.3469352837518893 & 0.7500692744202552 \\
\hline 6531014951216872 & 0.0001317176313091 & 0.7497415238236971 \\
\hline 5002536292007996 & 0.1533180741486575 & 13359221 \\
\hline 3968847566890 & 91186823 & 679718 \\
\hline 8828350 & 0.500 & 47565282 \\
\hline 0.0000033099404275 & 0.6530789090708981 & 3592249947 \\
\hline 0.8469208376826656 & 0.8471489549606462 & 0.7495705069454821 \\
\hline 30725515023031 & 0.4998809420072680 & 1074 \\
\hline 0.4998616593307978 & 0.6530328527894128 & 0.749 \\
\hline 0.3192843897157572 & 0.4867797240355922 & 0.9163086373993043 \\
\hline .1668085603331125 & 0.1803825150788025 & 0.9161564992090691 \\
\hline 0.0132398962200180 & 0.3331882006500743 & 0.91 \\
\hline 0.8198600515 & 0.4863978 & 6561 \\
\hline 0.6666908229553599 & 0.1804470759155525 & 0.9166111331437146 \\
\hline .5137740054084050 & 0.3338090310721586 & 986382 \\
\hline 32 & 0.9 & \\
\hline .1669645031271291 & 0.6804938712031366 & 0.9163393632337861 \\
\hline 0.0131398143310022 & 0.8332553608783009 & 0.9159735220213120 \\
\hline $.82034332 \mathrm{c}$ & 0.987348 & 0.91 \\
\hline .6684370412372920 & 0.680757150 & 0.91 \\
\hline .5141580030541931 & 0.8336664378090858 & 0.9156541671064541 \\
\hline .1668715934222857 & 0.3343206105175665 & 0.0208119256236669 \\
\hline .6672043287649173 & 0.3341028434747369 & 0.0218976386689325 \\
\hline .1665627145635546 & 0.8340134638931460 & 0.0202741500122934 \\
\hline 0.6678138218966686 & 0.8339765605942283 & 0.0198263084356461 \\
\hline 0.0000312894991189 & 0.0000487088667445 & 0.1461497506626485 \\
\hline 45000702000 & -0.0021914977185070 & 0.1453549 \\
\hline .0036727798233245 & 0.5031465089508335 & 0.1471329155128422 \\
\hline 0.4969283471601493 & 0.5019995392500566 & 0.1465666310716976 \\
\hline 3344150762237307 & 0.1673053696337763 & 0.1874168892375196 \\
\hline 8330933986775881 & 0.1675963515671829 & 0.1871466403087672 \\
\hline 0.3376520229252710 & 0.6671502112757510 & 0.1885588263890579 \\
\hline .8317371538635885 & 0.6650934455602068 & 0.1870576570315186 \\
\hline .1666359688159629 & 0.3325252533120870 & 0.3126114475112980 \\
\hline 0.6667422954134856 & 0.3328545807635965 & 0.3127930605783986 \\
\hline 0.1652838703942709 & 0.8317265486416150 & 0.3117059863718958 \\
\hline 0.6666481562890331 & 0.8338269486133064 & 0.3121875822795406 \\
\hline 0.000007506746410 & . 0003551224596866 & 0.3538346906861075 \\
\hline 0.5001093500371164 & -0.0005420685486879 & 0.3531022735570275 \\
\hline .0003701118843994 & 0.5011660673225227 & 0.3536376836518344 \\
\hline 49828321361114c & 0.497890617677851 & 0.353657237062700 \\
\hline
\end{tabular}




$\begin{array}{cll}0.3332639973678993 & 0.1655453733811191 & 0.4796116566847139 \\ 0.8335728265113410 & 0.1670648616954540 & 0.4792839499108558 \\ 0.3332416605186200 & 0.6664938015523643 & 0.4785261089250594 \\ 0.8323641917660833 & 0.6663645898681753 & 0.4794708781243160 \\ 0.1669195866951354 & 0.3331066444247300 & 0.5204963220178235 \\ 0.6666056142736816 & 0.3332454901086306 & 0.5203204833814141 \\ 0.1660880074269769 & 0.8328854304083420 & 0.5201346624958915 \\ 0.6664839085128968 & 0.8335559837732971 & 0.5201712205211101 \\ -0.0001879240812603 & -0.0001100439464554 & 0.6460322068455957 \\ 0.5000352494393369 & 0.0000138412734538 & 0.6460426870721599 \\ -0.0000736693854934 & 0.4999760703598382 & 0.6460965068698928 \\ 0.5000372389218162 & 0.4999006222966720 & 0.6461473402886220 \\ 0.3333331015881238 & 0.1666752275893721 & 0.6874029664282384 \\ 0.8334045815611612 & 0.1668341278499643 & 0.6872630228391917 \\ 0.3332460683119803 & 0.6666243396504840 & 0.6869196040150074 \\ 0.8332924074015842 & 0.6666202106206539 & 0.6873501122148944 \\ 0.1668210642133878 & 0.3336604616530501 & 0.8130632967338742 \\ 0.6667178812480618 & 0.3334951493407611 & 0.8131991249237243 \\ 0.1668685380221367 & 0.8333810105347382 & 0.8128507276997466 \\ 0.6665590395547113 & 0.8333388684809691 & 0.8125570506653629 \\ 0.0001683137027034 & 0.0002285136297696 & 0.8538026013882104 \\ 0.5000005140640470 & 0.0001417230555826 & 0.8541162122245093 \\ 0.0005272890201632 & 0.5004778505893517 & 0.8542827047796479 \\ 0.4996653366332498 & 0.5003305147312374 & 0.8543913775575532 \\ 0.3335205269644904 & 0.1672215299574986 & 0.9795518001093745 \\ 0.8333999204704463 & 0.1671316252419470 & 0.9797218243848020 \\ 0.3344584907096519 & 0.6683468630124771 & 0.9821348151368329 \\ 0.8333030805986977 & 0.6665219397950024 & 0.9796130738634037\end{array}$

(1.8) $2 \times 2 \times 1$ supercell with the EP localized on Fe8 $(-823.203 \mathrm{eV})$

$\begin{array}{lll}0.1804688210289438 & 0.0135428121035045 & 0.0830392863644579 \\ 0.3329064699828747 & 0.3195175050980105 & 0.0831679212680871 \\ 0.4857211288452994 & 0.1657051293482581 & 0.0828733256244318 \\ 0.6796210162550848 & 0.0137765212946428 & 0.0827588519216891 \\ 0.8330542285894211 & 0.3192903201286571 & 0.0827935420517156 \\ 0.9862056228194790 & 0.1662482962312637 & 0.0836118757216322 \\ 0.1792329718097773 & 0.5134981425077173 & 0.0835745777098622 \\ 0.3332085128536234 & 0.8198535404892963 & 0.0830938128629977 \\ 0.4856945753510042 & 0.6665581300187542 & 0.0826674417360239 \\ 0.6740081641521453 & 0.5090829130859811 & 0.0790746525210373 \\ 0.8342013714895727 & 0.8251666935306393 & 0.0785102136478971 \\ 0.9865952057657720 & 0.6667189322025792 & 0.0827395733024040 \\ 0.1558548146834787 & 0.1568356510791357 & 0.2503819810174968 \\ 0.0011771328513586 & 0.3450693757649331 & 0.2500673891825433\end{array}$




\begin{tabular}{|c|c|c|}
\hline 4 & 0.001 & \\
\hline 68 & 1519337547170364 & 0.250 \\
\hline 5008530869455493 & 3470521645178534 & \\
\hline 0 & 44 & 38 \\
\hline 035 & 85 & 186 \\
\hline 35 & 949 & 972 \\
\hline & & \\
\hline & & \\
\hline 96 & 0. & \\
\hline 0005 & 0.49316502 & \\
\hline & & \\
\hline & & \\
\hline 0.1668849 & 0.48 & 324 \\
\hline 73 & 0.18 & 397 \\
\hline & & \\
\hline 32 & 0. & \\
\hline 0.0141 & 0.68 & 0151 \\
\hline 11 & 0.8 & 0. \\
\hline & & \\
\hline 72 & 0.6 & 628 \\
\hline 163 & 0.83 & 0. \\
\hline 77 & 0.9 & \\
\hline 30 & & \\
\hline 120 & 0.16 & 0.5 \\
\hline 804 & 0.01 & 49 \\
\hline 1 & 0.3 & \\
\hline 38 & 0.16 & \\
\hline 811 & 1482 & 5684 \\
\hline 0.486 & 0.81 & 04 \\
\hline & & \\
\hline 04 & 0.51 & 0.58 \\
\hline 932836 & 0.81 & 0.5 \\
\hline & & \\
\hline & 0.513 & \\
\hline 0.3465306285826952 & 0.34685811 & 0.749885242 \\
\hline 0.1529726450489797 & 0000488516690007 & 0.7500795400673105 \\
\hline & 0.15 & 81 \\
\hline 0.8468485790677722 & 0.3468218116927292 & 0.7499268985436197 \\
\hline 0.6527641068640824 & 308824804193 & 2380396 \\
\hline 39 & 0.15 & 0.749 \\
\hline 26929674933 & 0.8467149235526712 & 0.7498594333469353 \\
\hline 0.1529758316190497 & 0.4998319017373967 & 0.75005 \\
\hline 68540 & 0.6529239461977 & 0.7497 \\
\hline 2400 & $040014 / 52050$ & 0.149039404505000 \\
\hline
\end{tabular}




\begin{tabular}{|c|c|c|}
\hline 49 & 3451 & 0. \\
\hline 0.4997175872997600 & 0.6528426827021949 & 0.7496996465449582 \\
\hline 3193913517546503 & 0.4860887271454116 & 0.9166461369394704 \\
\hline 1659698133031422 & 0.1798789770581722 & 0.9166525331182731 \\
\hline .0134890958000972 & 0.3334053292135836 & 0.91662316 \\
\hline ).8192695811180881 & 0.4863418254871648 & 0.9162198327410226 \\
\hline 19147410 & 35195516478 & 5347 \\
\hline 01 & 2978339126 & 08350 \\
\hline 0.3194042434843170 & 0.9862594750061152 & 49410 \\
\hline 1666762324193947 & 0.6799497964136522 & 0.9162036088418904 \\
\hline 30980498931549 & 0.8325210061275211 & 0.91 \\
\hline 8190291571776372 & 0.9876009371156315 & 0.914 \\
\hline 0.6661104003628902 & 0.6803641068227833 & 0.9155826370470976 \\
\hline 0.5124773552839892 & 0.8328782999788235 & 0.9159654334553238 \\
\hline 379606749021 & 0.3329867629187023 & \\
\hline 0.665865 & 4777 & 0630 \\
\hline 1656319770956418 & 0.8325831440420707 & 0.0208186978465377 \\
\hline .6658358434587768 & 0.8337773887819006 & 010742 \\
\hline & 0.9 & \\
\hline 0.4999376066118918 & 0.0000333695679439 & 0.14616000 \\
\hline 0.9969271343252100 & 0.5004815629716193 & 0.1470615540536633 \\
\hline .5021943323295853 & 0.5 & 0.14 \\
\hline .332 & 68402487001 & 0.18 \\
\hline 0.8349139226347322 & 0.1667457167640627 & 0.1870697666007205 \\
\hline 0.3324893501110510 & 0.6656005637302139 & 0.1872586265264312 \\
\hline .8329067578619196 & 0.6703761322080756 & 0.188455 \\
\hline .1672812731416923 & 0.3339852672239739 & 0.3128243 \\
\hline 0.6683928192687707 & 0.3336502507244524 & 0.3117025426986266 \\
\hline 0.1675502335497231 & 0.8342315952522285 & 0.3126591324025139 \\
\hline 6663751499718568 & 0.8329199368440757 & 0.31220479 \\
\hline .0022388726488372 & 0.0004808190941127 & 0.3536796345965154 \\
\hline 0.5004898881228304 & 0.0003778936429616 & 0.3538133694753137 \\
\hline 9990113134176180 & 0.4993838974557756 & 0.3536527209537861 \\
\hline 5007132402621728 & 0.5007057020307814 & 0.3530365080072902 \\
\hline 0.3345923750155301 & 0.1677609120888284 & 0.4796188531615834 \\
\hline ).8337447709329205 & 0.1661182652439527 & 0.4794451512882382 \\
\hline .3332134917733420 & 0.6666301006874562 & 0.4792778217468046 \\
\hline 0.8336692182138871 & 0.6668584579624424 & 0.4786122507546806 \\
\hline 0.1668718024812005 & 0.3334279867854192 & 0.5203508927393228 \\
\hline 0.6672258424135079 & 0.3332611934751053 & 0.5201283326331304 \\
\hline 0.1670640181158571 & 0.8338662076117315 & 0.5205455008341240 \\
\hline 0.6665885155978422 & 0.8330411909561160 & 0.5201937019250958 \\
\hline .0001796911878053 & 0.0001387283451024 & 0.6461336654102745 \\
\hline 50015874207570 & 0.999917767835410 & 0.646064989261854 \\
\hline
\end{tabular}




$\begin{array}{lll}0.0000678709437484 & 0.4999490995617708 & 0.6460891643929663 \\ 0.5000223022642057 & 0.5000012923189769 & 0.6459677131406507 \\ 0.3332814219750445 & 0.1666133771284754 & 0.6874365300961125 \\ 0.8333568585470990 & 0.1666290252633196 & 0.6873311467471304 \\ 0.3330605610876027 & 0.6665272553794210 & 0.6872695153147012 \\ 0.8333409754613964 & 0.6665766274059204 & 0.6869707783223560 \\ 0.1663764655436639 & 0.3331454634449986 & 0.8132328149606485 \\ 0.6665080589483003 & 0.3334061448136708 & 0.8127768218847098 \\ 0.1661895928097746 & 0.8330724575089173 & 0.8130602633273963 \\ 0.6664905753104324 & 0.8330783323399571 & 0.8124691185574383 \\ 0.9994619013479793 & 0.9992979009182145 & 0.8543784194700225 \\ 0.4995910741060686 & 0.9998422240628565 & 0.8538834903211870 \\ 0.9993721240614637 & 0.4999387439532892 & 0.8542842659439510 \\ 0.4996706868084139 & 0.4997163369535984 & 0.8540624803207639 \\ 0.3326499609662962 & 0.1662314425048197 & 0.9796235472810000 \\ 0.8333647248481675 & 0.1667078296625477 & 0.9796436692058946 \\ 0.3326696980058504 & 0.6661220926228000 & 0.9797990766856863 \\ 0.8315037952475884 & 0.6660731208148113 & 0.9820383429411141\end{array}$

(1.9) $2 \times 2 \times 1$ supercell with the EP transfer from initial to final state with $d_{\mathrm{Fe}-\mathrm{Fe}}=2.91 \AA$ as an example Initial coordinate: $0(-823.203 \mathrm{eV})$

$\begin{array}{lll}0.1775296638988950 & 0.0098864594904548 & 0.0828756767353683 \\ 0.3237655789899154 & 0.3086449919922368 & 0.0810497242043729 \\ 0.4867062314112072 & 0.1672482587193708 & 0.0834417002043438 \\ 0.6797044910517636 & 0.0146507356987682 & 0.0833828900821327 \\ 0.8341416689254200 & 0.3200373828950028 & 0.0833142708917892 \\ 0.9846867444401258 & 0.1652156433799164 & 0.0829975223989163 \\ 0.1795423752553227 & 0.5129228529914100 & 0.0831521626640514 \\ 0.3320915512990337 & 0.8215750834534763 & 0.0833208442786446 \\ 0.4834659313677361 & 0.6735489851535839 & 0.0819554139040291 \\ 0.6853848919500241 & 0.5141544765727362 & 0.0831011367232782 \\ 0.8325490271359929 & 0.8196104327404483 & 0.0837648817504069 \\ 0.9873586119486656 & 0.6667772493935004 & 0.0833941155018580 \\ 0.1528792971052447 & 0.1532647710212051 & 0.2502385520357527 \\ 0.0001361795970754 & 0.3469022916067299 & 0.2502199740925874 \\ 0.3471338494210391 & 0.0005587663780779 & 0.2497244969112869 \\ 0.6537020262266630 & 0.1529650165925935 & 0.2505471755176473 \\ 0.4991485772838358 & 0.3415963690244834 & 0.2548001824126955 \\ 0.8476218552150809 & 0.0010533605042919 & 0.2505017298924438 \\ 0.154114558922263 & 0.6532367611675850 & 0.2498293369883546 \\ 0.0004659752327347 & 0.8472443708833877 & 0.2501553048215922 \\ 0.3467433995666365 & 0.5000738319695621 & 0.2505474682227289 \\ 0.6592869786614727 & 0.6577158945093013 & 0.2542094907475846 \\ 0.5002228521719964 & 0.8474922673814852 & 0.2506089449092809\end{array}$




\begin{tabular}{|c|c|c|}
\hline & & \\
\hline 965 & 055377 & \\
\hline & & \\
\hline & & \\
\hline & & \\
\hline & & \\
\hline 69 & 49 & \\
\hline & & \\
\hline & & \\
\hline 23 & 10 & \\
\hline & & \\
\hline & & \\
\hline & & \\
\hline 0.48 & 0.31 & \\
\hline & & \\
\hline & & \\
\hline 93 & 14 & 28 \\
\hline 0.68 & & \\
\hline 0.8 & & \\
\hline & & \\
\hline 4 & & \\
\hline 0.3 & & \\
\hline & & \\
\hline & & \\
\hline 0.83 & 66 & \\
\hline 0.34 & 01 & \\
\hline & & \\
\hline & & \\
\hline 1216 & 0286 & \\
\hline 0.652 & & \\
\hline & & \\
\hline & & \\
\hline 0.15 & 17 & \\
\hline 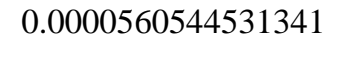 & & \\
\hline & & \\
\hline 0.6 & 3 & S \\
\hline & & \\
\hline & & \\
\hline & & \\
\hline 0.01 & 0.332 & 04 \\
\hline & & \\
\hline & 1 & 91 \\
\hline 0.51329261042527 & 0.3330014424202 & 0.916600888901 \\
\hline & & \\
\hline
\end{tabular}




\begin{tabular}{|c|c|c|}
\hline 64 & 154 & 0. \\
\hline 0.0124056449156965 & 0.8323422885269557 & 0.9160262775081687 \\
\hline 8193150413586877 & 0.9863633455343671 & 0.9162751094278396 \\
\hline 60209802785088 & 0.6799535528698977 & 0.9169612669398175 \\
\hline 6690824880 & 0.8341256034681285 & 0.9154734 \\
\hline 30607 & 0.3324253209601774 & 0.02 \\
\hline 95 & 95 & 0.02 \\
\hline 6315 & 54478 & \\
\hline 7204 & 0.832 & 0.021 \\
\hline 0007255344138573 & 0.0003982207279100 & 0.1458685292212673 \\
\hline 84103658551758 & 0.0000012884012070 & 6655 \\
\hline 5889059 & 0.5010443652462268 & 0.146 \\
\hline 0.5004678184311419 & 0.4963628397954032 & 0.1448408184772301 \\
\hline 0.3355250975 & 0.1719380382540623 & 0.1866857791769887 \\
\hline 308 & 0.16 & \\
\hline 0.33 & 0.66 & 630 \\
\hline 0.8311514842868845 & 0.6659029296524336 & 0.1880434909416791 \\
\hline 1677468494337253 & 0.3342376406521909 & 0.3124984 \\
\hline 7 & & \\
\hline 0.1675166167168471 & 0.8338068924952748 & 0.3114477422137347 \\
\hline 0.6674292569975158 & 0.8343113565719164 & 0.3131796530508686 \\
\hline 0.000651 & 0.0005 & 0.35 \\
\hline 87240 & 0.000093029157 & 0.353708 \\
\hline 0.0006275410552356 & 0.5006528064440082 & 0.3535606797177064 \\
\hline 0.5018529525503587 & 0.5007339325229684 & 0.3512350424245297 \\
\hline 33379312766 & 08463 & 0.4789 \\
\hline ).8337104418571557 & 0.1669269940088066 & 0.4794718391 \\
\hline 0.3339570214894712 & 0.6668478707743578 & 0.4790513362604233 \\
\hline 0.8336153708549686 & 0.6670775601737631 & 0.4792613949473643 \\
\hline 政 & 0.3336894504866 & $0.520250 c$ \\
\hline .6667875980951681 & 0.3336323032634859 & 0.5208337791220042 \\
\hline 0.1668947176160828 & 0.8335670420349146 & 0.5200942366772657 \\
\hline 6667714498586370 & 0.8333032266435788 & 0.5205800475749099 \\
\hline .0000097631618688 & 0.0000422257116815 & 0.6459223868329012 \\
\hline 0.4999390031365915 & 0.0000263768424713 & 0.6459973324358805 \\
\hline 0.0002308008321663 & 0.5001029500990144 & 0.6460280147125723 \\
\hline .4999693463304537 & 0.5000645874411851 & 0.6463751552508293 \\
\hline 0.3331355202075558 & 0.1664159313585710 & 0.6871669689467332 \\
\hline 0.8331065435251372 & 0.1666074343043052 & 0.6872732068786471 \\
\hline 0.3332458045117614 & 0.6666181302590317 & 0.6872572126401315 \\
\hline 0.8333110929339359 & 0.6666025555827786 & 0.6873615556322887 \\
\hline 0.1662774574911925 & 0.3326399350664311 & 0.8127695584683217 \\
\hline .6667842309186296 & 0.3335152724278619 & 0.813091467626124 \\
\hline 1664865289154 & 0.833121819322555 & 0.8129802157728 \\
\hline
\end{tabular}




$\begin{array}{ccc}0.6660807384389682 & 0.8332598137252860 & 0.8132739493633726 \\ -0.0012399719726091 & -0.0012762328088864 & 0.8537335480056757 \\ 0.4996265554645943 & 0.0004122072708792 & 0.8538943488150317 \\ 0.0001889714992081 & 0.4999111558555656 & 0.8540467979969809 \\ 0.4996436641813404 & 0.4996675323490507 & 0.8547484041180669 \\ 0.3311683005899825 & 0.1660942928734377 & 0.9795771768475786 \\ 0.8328920031905034 & 0.1661190061694215 & 0.9795323169220234 \\ 0.3343067467317206 & 0.6672475136796856 & 0.9797520078929414 \\ 0.8325931793074005 & 0.6657843976323746 & 0.9802951296436833\end{array}$

Interpolating coordinate: $0.167(-823.188 \mathrm{eV})$

$\begin{array}{lcl}0.176927357 & 0.009762812 & 0.082210468 \\ 0.325465420 & 0.311397072 & 0.080597592 \\ 0.486658780 & 0.167158225 & 0.083287001 \\ 0.679601487 & 0.014472060 & 0.083365042 \\ 0.833965386 & 0.320019494 & 0.083222278 \\ 0.984825686 & 0.165430354 & 0.082896506 \\ 0.179528582 & 0.513067074 & 0.083056169 \\ 0.332236475 & 0.821204307 & 0.083193223 \\ 0.483892595 & 0.672330463 & 0.082197285 \\ 0.684527666 & 0.514042025 & 0.083049434 \\ 0.832567002 & 0.819598412 & 0.083617794 \\ 0.987049833 & 0.666608149 & 0.083268457 \\ 0.152049914 & 0.153161517 & 0.250195368 \\ -0.000008711 & 0.346889893 & 0.250136009 \\ 0.347594444 & -0.000638782 & 0.249966730 \\ 0.653735988 & 0.153137488 & 0.250439311 \\ 0.500903505 & 0.344374927 & 0.254328659 \\ 0.847348612 & 0.000886905 & 0.250367111 \\ 0.154030934 & 0.653064891 & 0.249822595 \\ 0.000522544 & 0.847245584 & 0.250019201 \\ 0.346756566 & 0.500020092 & 0.250404866 \\ 0.658712536 & 0.657606415 & 0.253537156 \\ 0.500393043 & 0.847132924 & 0.250480189 \\ 0.847806824 & 0.500438564 & 0.250556947 \\ 0.013864750 & 0.180557822 & 0.417094156 \\ 0.320240601 & 0.334241284 & 0.416926975 \\ 0.166696470 & 0.486853276 & 0.417058243 \\ 0.514268973 & 0.179494371 & 0.418148974 \\ 0.820851933 & 0.333948689 & 0.417315507 \\ 0.667423982 & 0.486680474 & 0.417792515 \\ 0.013947146 & 0.680652669 & 0.416718384 \\ 0.319837648 & 0.833298813 & 0.416916572 \\ 0.167312990 & 0.986929044 & 0.416585768\end{array}$




$\begin{array}{rrr}0.514063289 & 0.680466848 & 0.417091017 \\ 0.821430904 & 0.835269562 & 0.418129583 \\ 0.666723755 & 0.986953357 & 0.417323563 \\ 0.486451469 & 0.319897112 & 0.583572464 \\ 0.180308405 & 0.166755829 & 0.583209721 \\ 0.333271817 & 0.013575266 & 0.583353227 \\ 0.986749433 & 0.319927528 & 0.583440089 \\ 0.680473975 & 0.166966467 & 0.583704606 \\ 0.833237506 & 0.013722003 & 0.583585460 \\ 0.486489699 & 0.819797788 & 0.583460129 \\ 0.180331061 & 0.666834797 & 0.583302289 \\ 0.333379386 & 0.513668920 & 0.583521185 \\ 0.986802746 & 0.819865723 & 0.583431770 \\ 0.680323564 & 0.666846108 & 0.583692786 \\ 0.833569084 & 0.513842314 & 0.583637937 \\ 0.346770360 & 0.346721990 & 0.749753439 \\ 0.152769367 & -0.000472704 & 0.749459577 \\ -0.000276703 & 0.152821362 & 0.749406645 \\ 0.846699653 & 0.346878427 & 0.749711322 \\ 0.652873593 & -0.000115026 & 0.749719127 \\ 0.499992249 & 0.153250115 & 0.749720470 \\ 0.346651366 & 0.846893996 & 0.749596811 \\ 0.152991686 & 0.499775457 & 0.749694747 \\ 0.000015882 & 0.652977744 & 0.749688458 \\ 0.846288094 & 0.846627674 & 0.749777864 \\ 0.653019740 & 0.499990242 & 0.750013974 \\ 0.499804485 & 0.652965260 & 0.749976154 \\ 0.319142752 & 0.486176422 & 0.916090494 \\ 0.165294278 & 0.179273573 & 0.914902683 \\ 0.012518471 & 0.332567364 & 0.915929750 \\ 0.819748570 & 0.486277894 & 0.916012010 \\ 0.666667470 & 0.180133707 & 0.916218991 \\ 0.513212378 & 0.332898488 & 0.916514188 \\ 0.319160760 & 0.986217626 & 0.915981263 \\ 0.166426273 & 0.679919540 & 0.916111215 \\ 0.012257750 & 0.832180336 & 0.915822692 \\ 0.819274821 & 0.986311489 & 0.916277967 \\ 0.665971284 & 0.679921155 & 0.916859927 \\ 0.513454138 & 0.833982614 & 0.915617651 \\ 0.165825233 & 0.332648048 & 0.020458428 \\ 0.666817300 & 0.333502599 & 0.020991823 \\ 0.165946748 & 0.832590407 & 0.020426361 \\ 0.665096189 & 0.832962938 & 0.021701079 \\ 0.000945429 & -0.000184625 & 0.145726150\end{array}$




$\begin{array}{rcc}0.498156712 & 0.000103133 & 0.146357508 \\ 0.000715119 & 0.500888748 & 0.146086499 \\ 0.499997555 & 0.496111169 & 0.145096341 \\ 0.335080196 & 0.171707450 & 0.186942536 \\ 0.833242832 & 0.166524295 & 0.187135134 \\ 0.336164917 & 0.666431750 & 0.186413000 \\ 0.831386939 & 0.666136497 & 0.187904544 \\ 0.167518613 & 0.334068973 & 0.312409939 \\ 0.667515358 & 0.333218248 & 0.313369160 \\ 0.167670986 & 0.833826377 & 0.311443512 \\ 0.667420927 & 0.834305461 & 0.313088148 \\ 0.000689133 & 0.000673149 & 0.353555614 \\ 0.499806403 & 0.000007241 & 0.353657790 \\ 0.000611070 & 0.500661633 & 0.353563886 \\ 0.501936003 & 0.500743392 & 0.351607627 \\ 0.333790851 & 0.167455372 & 0.478826343 \\ 0.833639771 & 0.166926351 & 0.479399881 \\ 0.333938842 & 0.666768931 & 0.479084332 \\ 0.833799331 & 0.667238468 & 0.479282126 \\ 0.167039086 & 0.333619163 & 0.520199169 \\ 0.666841525 & 0.333714089 & 0.520742709 \\ 0.166974576 & 0.833556093 & 0.520059970 \\ 0.666805503 & 0.833366627 & 0.520500727 \\ -0.000003115 & 0.000046165 & 0.645888567 \\ 0.499957659 & 0.000032747 & 0.645968643 \\ 0.000223374 & 0.500102129 & 0.646006424 \\ 0.499996871 & 0.500093164 & 0.646294134 \\ 0.333137336 & 0.166446005 & 0.687091344 \\ 0.833086293 & 0.166602014 & 0.687235510 \\ 0.333239715 & 0.666631812 & 0.687237315 \\ 0.833277011 & 0.666610460 & 0.687333434 \\ 0.166283361 & 0.332718135 & 0.812688505 \\ 0.666635406 & 0.333422138 & 0.813035394 \\ 0.166445082 & 0.833159511 & 0.812920870 \\ 0.666087348 & 0.833228448 & 0.813220335 \\ -0.001128721 & -0.001112333 & 0.853741820 \\ 0.499528672 & 0.000310784 & 0.853918309 \\ 0.000039844 & 0.499881554 & 0.853987006 \\ 0.499569404 & 0.499579299 & 0.854646929 \\ 0.331178905 & 0.166073907 & 0.979955764 \\ 0.832811159 & 0.166110319 & 0.979524630 \\ 0.334099073 & 0.667137165 & 0.979698933 \\ 0.832549978 & 0.665852691 & 0.980135964 \\ & & \end{array}$


Interpolating coordinate: $0.333(-823.145 \mathrm{eV})$

$\begin{array}{ccc}0.176325049 & 0.009639164 & 0.081545260 \\ 0.327165261 & 0.314149151 & 0.080145460 \\ 0.486611329 & 0.167068192 & 0.083132301 \\ 0.679498484 & 0.014293384 & 0.083347194 \\ 0.833789104 & 0.320001604 & 0.083130285 \\ 0.984964627 & 0.165645064 & 0.082795491 \\ 0.179514788 & 0.513211295 & 0.082960176 \\ 0.332381400 & 0.820833531 & 0.083065603 \\ 0.484319258 & 0.671111942 & 0.082439155 \\ 0.683670440 & 0.513929574 & 0.082997732 \\ 0.832584977 & 0.819586392 & 0.083470705 \\ 0.986741054 & 0.666439048 & 0.083142799 \\ 0.151220531 & 0.153058263 & 0.250152185 \\ -0.000153602 & 0.346877494 & 0.250052045 \\ 0.348055038 & -0.001836330 & 0.250208963 \\ 0.653769949 & 0.153309960 & 0.250331446 \\ 0.502658433 & 0.347153485 & 0.253857135 \\ 0.847075368 & 0.000720449 & 0.250232493 \\ 0.153947309 & 0.652893021 & 0.249815852 \\ 0.000579114 & 0.847246797 & 0.249883098 \\ 0.346769733 & 0.499966351 & 0.250262264 \\ 0.658138094 & 0.657496936 & 0.252864821 \\ 0.500563233 & 0.846773582 & 0.250351434 \\ 0.847968861 & 0.500684969 & 0.250452448 \\ 0.013808940 & 0.180561869 & 0.417089493 \\ 0.320225307 & 0.334159287 & 0.416831139 \\ 0.166708643 & 0.486823683 & 0.417003416 \\ 0.514265005 & 0.179795692 & 0.417861948 \\ 0.820855227 & 0.334014023 & 0.417268521 \\ 0.667611819 & 0.486907837 & 0.417873688 \\ 0.013993753 & 0.680615970 & 0.416750226 \\ 0.319854333 & 0.833190643 & 0.417077890 \\ 0.167328828 & 0.986912135 & 0.416492575 \\ 0.514099319 & 0.680494975 & 0.417100122 \\ 0.821364338 & 0.835119471 & 0.417924334 \\ 0.666768544 & 0.986945060 & 0.417248247 \\ 0.486471928 & 0.319905030 & 0.583507231 \\ 0.180296512 & 0.166738069 & 0.583171275 \\ 0.333330233 & 0.013617267 & 0.583293251 \\ 0.986727040 & 0.319920972 & 0.583426451 \\ 0.486532328320 & 0.166971981 & 0.583633755 \\ & 0.013736597 & 0.583547255 \\ 0.8198045866 & 0.583478377\end{array}$




$\begin{array}{rrr}0.180359550 & 0.666828859 & 0.583342682 \\ 0.333349391 & 0.513608198 & 0.583479318 \\ 0.986871752 & 0.819919474 & 0.583415188 \\ 0.680379776 & 0.666921669 & 0.583676027 \\ 0.833580152 & 0.513859053 & 0.583658151 \\ 0.346741947 & 0.346737895 & 0.749684287 \\ 0.152791286 & -0.000402237 & 0.749448350 \\ -0.000297331 & 0.152828792 & 0.749414946 \\ 0.846628633 & 0.346843966 & 0.749694889 \\ 0.652852929 & -0.000132563 & 0.749729996 \\ 0.499925077 & 0.153173727 & 0.749680422 \\ 0.346647612 & 0.846881261 & 0.749621902 \\ 0.152915060 & 0.499782021 & 0.749639992 \\ -0.000024291 & 0.652972817 & 0.749664787 \\ 0.846299870 & 0.846675340 & 0.749747923 \\ 0.652962185 & 0.499968494 & 0.749967844 \\ 0.499824438 & 0.652995111 & 0.749912446 \\ 0.319073787 & 0.486450687 & 0.915815962 \\ 0.165416450 & 0.179482077 & 0.914988871 \\ 0.012450186 & 0.332610097 & 0.915885053 \\ 0.819618869 & 0.486268084 & 0.916005164 \\ 0.666627280 & 0.180085972 & 0.916152236 \\ 0.513132146 & 0.332795534 & 0.916427488 \\ 0.319129575 & 0.986228284 & 0.915994133 \\ 0.166392333 & 0.679882387 & 0.916053222 \\ 0.012109855 & 0.832018383 & 0.915619106 \\ 0.819234600 & 0.986259632 & 0.916280825 \\ 0.665921588 & 0.679888756 & 0.916758588 \\ 0.513396607 & 0.833839625 & 0.915761825 \\ 0.165789128 & 0.332870775 & 0.020276041 \\ 0.666533787 & 0.333312041 & 0.020899658 \\ 0.165892347 & 0.832563164 & 0.020342982 \\ 0.665180235 & 0.832959628 & 0.021688194 \\ 0.001165323 & 0.000028971 & 0.145583771 \\ 0.497903059 & 0.000204977 & 0.146451305 \\ 0.000533691 & 0.500733130 & 0.146051979 \\ 0.499527292 & 0.495859498 & 0.145351864 \\ 0.334635295 & 0.171476862 & 0.187199293 \\ 0.833084027 & 0.166374953 & 0.187093724 \\ 0.335916858 & 0.666532001 & 0.186509106 \\ 0.831622393 & 0.666370063 & 0.187765598 \\ 0.167290376 & 0.333900304 & 0.312321431 \\ 0.667519037 & 0.333462408 & 0.313172362 \\ 0.167825356 & 0.833845862 & 0.311439283\end{array}$




$\begin{array}{rrr}0.667412596 & 0.834299565 & 0.312996644 \\ 0.000726444 & 0.000754085 & 0.353403115 \\ 0.499659751 & -0.000078547 & 0.353606963 \\ 0.000594600 & 0.500670459 & 0.353567092 \\ 0.502019053 & 0.500752851 & 0.351980212 \\ 0.333788574 & 0.167396481 & 0.478727898 \\ 0.833569101 & 0.166925708 & 0.479327922 \\ 0.333920662 & 0.6666689990 & 0.479117327 \\ 0.833983291 & 0.667399376 & 0.479302857 \\ 0.166962199 & 0.333548875 & 0.520148259 \\ 0.666895452 & 0.333795875 & 0.520651638 \\ 0.167054434 & 0.833545144 & 0.520025703 \\ 0.666839556 & 0.833430028 & 0.520421406 \\ -0.000015994 & 0.000050105 & 0.645854746 \\ 0.499976315 & 0.000039117 & 0.645939953 \\ 0.000215948 & 0.500101308 & 0.645984833 \\ 0.500024395 & 0.500121741 & 0.646213112 \\ 0.333139152 & 0.166476079 & 0.687015720 \\ 0.833066042 & 0.166596593 & 0.687197813 \\ 0.333233626 & 0.666645494 & 0.687217417 \\ 0.833242929 & 0.6666618364 & 0.687305313 \\ 0.166289264 & 0.332796335 & 0.812607452 \\ 0.666486581 & 0.333329003 & 0.812979320 \\ 0.166403635 & 0.833197203 & 0.812861524 \\ 0.666093958 & 0.833197083 & 0.813166721 \\ -0.001017470 & -0.000948432 & 0.853750093 \\ 0.499430788 & 0.000209361 & 0.853942270 \\ -0.000109284 & 0.499851952 & 0.853927215 \\ 0.499495144 & 0.499491067 & 0.854545455 \\ 0.331189510 & 0.166053522 & 0.980334351 \\ 0.832730314 & 0.166101632 & 0.979516944 \\ 0.333891399 & 0.667026816 & 0.979645858 \\ 0.832506777 & 0.665920984 & 0.979976798 \\ & & \end{array}$

Interpolating coordinate: $0.417(-823.113 \mathrm{eV})$

$\begin{array}{lll}0.176325049 & 0.009639164 & 0.081545260 \\ 0.327165261 & 0.314149151 & 0.080145460 \\ 0.486611329 & 0.167068192 & 0.083132301 \\ 0.679498484 & 0.014293384 & 0.083347194 \\ 0.833789104 & 0.320001604 & 0.083130285 \\ 0.984964627 & 0.165645064 & 0.082795491 \\ 0.179514788 & 0.513211295 & 0.082960176 \\ 0.332381400 & 0.820833531 & 0.083065603 \\ 0.484319258 & 0.671111942 & 0.082439155\end{array}$




$\begin{array}{rrr}0.683670440 & 0.513929574 & 0.082997732 \\ 0.832584977 & 0.819586392 & 0.083470705 \\ 0.986741054 & 0.666439048 & 0.083142799 \\ 0.151220531 & 0.153058263 & 0.250152185 \\ -0.000153602 & 0.346877494 & 0.250052045 \\ 0.348055038 & -0.001836330 & 0.250208963 \\ 0.653769949 & 0.153309960 & 0.250331446 \\ 0.502658433 & 0.347153485 & 0.253857135 \\ 0.847075368 & 0.000720449 & 0.250232493 \\ 0.153947309 & 0.652893021 & 0.249815852 \\ 0.000579114 & 0.847246797 & 0.249883098 \\ 0.346769733 & 0.499966351 & 0.250262264 \\ 0.658138094 & 0.657496936 & 0.252864821 \\ 0.500563233 & 0.846773582 & 0.250351434 \\ 0.847968861 & 0.500684969 & 0.250452448 \\ 0.013808940 & 0.180561869 & 0.417089493 \\ 0.320225307 & 0.334159287 & 0.416831139 \\ 0.166708643 & 0.486823683 & 0.417003416 \\ 0.514265005 & 0.179795692 & 0.417861948 \\ 0.820855227 & 0.334014023 & 0.417268521 \\ 0.667611819 & 0.486907837 & 0.417873688 \\ 0.013993753 & 0.680615970 & 0.416750226 \\ 0.319854333 & 0.833190643 & 0.417077890 \\ 0.167328828 & 0.986912135 & 0.416492575 \\ 0.514099319 & 0.680494975 & 0.417100122 \\ 0.821364338 & 0.835119471 & 0.417924334 \\ 0.666768544 & 0.986945060 & 0.417248247 \\ 0.486471928 & 0.319905030 & 0.583507231 \\ 0.180296512 & 0.166738069 & 0.583171275 \\ 0.333330233 & 0.013617267 & 0.583293251 \\ 0.986727040 & 0.319920972 & 0.583426451 \\ 0.680422883 & 0.166971981 & 0.583633755 \\ 0.833248247 & 0.013736597 & 0.583547255 \\ 0.486552320 & 0.819804586 & 0.583478377 \\ 0.180359550 & 0.666828859 & 0.583342682 \\ 0.333349391 & 0.513608198 & 0.583479318 \\ 0.986871752 & 0.819919474 & 0.583415188 \\ 0.680379776 & 0.666921669 & 0.583676027 \\ 0.833580152 & 0.513859053 & 0.583658151 \\ 0.346741947 & 0.346737895 & 0.749684287 \\ 0.152791286 & -0.000402237 & 0.749448350 \\ 0.000297331 & 0.152828792 & 0.749414946 \\ 0.846628633 & 0.346843966 & 0.749694889 \\ & -0.000132563 & 0.749729996\end{array}$




$\begin{array}{ccc}0.499925077 & 0.153173727 & 0.749680422 \\ 0.346647612 & 0.846881261 & 0.749621902 \\ 0.152915060 & 0.499782021 & 0.749639992 \\ -0.000024291 & 0.652972817 & 0.749664787 \\ 0.846299870 & 0.846675340 & 0.749747923 \\ 0.652962185 & 0.499968494 & 0.749967844 \\ 0.499824438 & 0.652995111 & 0.749912446 \\ 0.319073787 & 0.486450687 & 0.915815962 \\ 0.165416450 & 0.179482077 & 0.914988871 \\ 0.012450186 & 0.332610097 & 0.915885053 \\ 0.819618869 & 0.486268084 & 0.916005164 \\ 0.666627280 & 0.180085972 & 0.916152236 \\ 0.513132146 & 0.332795534 & 0.916427488 \\ 0.319129575 & 0.986228284 & 0.915994133 \\ 0.166392333 & 0.679882387 & 0.916053222 \\ 0.012109855 & 0.832018383 & 0.915619106 \\ 0.819234600 & 0.986259632 & 0.916280825 \\ 0.665921588 & 0.679888756 & 0.916758588 \\ 0.513396607 & 0.833839625 & 0.915761825 \\ 0.165789128 & 0.332870775 & 0.020276041 \\ 0.666533787 & 0.333312041 & 0.020899658 \\ 0.165892347 & 0.832563164 & 0.020342982 \\ 0.665180235 & 0.832959628 & 0.021688194 \\ 0.001165323 & 0.000028971 & 0.145583771 \\ 0.497903059 & 0.000204977 & 0.146451305 \\ 0.000533691 & 0.500733130 & 0.146051979 \\ 0.499527292 & 0.495859498 & 0.145351864 \\ 0.334635295 & 0.171476862 & 0.187199293 \\ 0.833084027 & 0.166374953 & 0.187093724 \\ 0.335916858 & 0.666532001 & 0.186509106 \\ 0.831622393 & 0.666370063 & 0.187765598 \\ 0.167290376 & 0.333900304 & 0.312321431 \\ 0.667519037 & 0.333462408 & 0.313172362 \\ 0.167825356 & 0.833845862 & 0.311439283 \\ 0.667412596 & 0.834299565 & 0.312996644 \\ 0.000726444 & 0.000754085 & 0.353403115 \\ 0.499659751 & -0.000078547 & 0.353606963 \\ 0.000594600 & 0.500670459 & 0.353567092 \\ 0.502019053 & 0.500752851 & 0.351980212 \\ 0.333788574 & 0.167396481 & 0.478727898 \\ 0.833569101 & 0.166925708 & 0.479327922 \\ 0.333920662 & 0.666689990 & 0.479117327 \\ 0.833983291 & 0.667399376 & 0.479302857 \\ 0.166962199 & 0.333548875 & 0.520148259\end{array}$




$\begin{array}{rcc}0.666895452 & 0.333795875 & 0.520651638 \\ 0.167054434 & 0.833545144 & 0.520025703 \\ 0.666839556 & 0.833430028 & 0.520421406 \\ -0.000015994 & 0.000050105 & 0.645854746 \\ 0.499976315 & 0.000039117 & 0.645939953 \\ 0.000215948 & 0.500101308 & 0.645984833 \\ 0.500024395 & 0.500121741 & 0.646213112 \\ 0.333139152 & 0.166476079 & 0.687015720 \\ 0.833066042 & 0.166596593 & 0.687197813 \\ 0.333233626 & 0.666645494 & 0.687217417 \\ 0.833242929 & 0.666618364 & 0.687305313 \\ 0.166289264 & 0.332796335 & 0.812607452 \\ 0.666486581 & 0.333329003 & 0.812979320 \\ 0.166403635 & 0.833197203 & 0.812861524 \\ 0.666093958 & 0.833197083 & 0.813166721 \\ -0.001017470 & -0.000948432 & 0.853750093 \\ 0.499430788 & 0.000209361 & 0.853942270 \\ -0.000109284 & 0.499851952 & 0.853927215 \\ 0.499495144 & 0.499491067 & 0.854545455 \\ 0.331189510 & 0.166053522 & 0.980334351 \\ 0.832730314 & 0.166101632 & 0.979516944 \\ 0.333891399 & 0.667026816 & 0.979645858 \\ 0.832506777 & 0.665920984 & 0.979976798\end{array}$

Interpolating coordinate: $0.433(-823.106 \mathrm{eV})$

$\begin{array}{rrr}0.175963665 & 0.009564975 & 0.081146135 \\ 0.328185166 & 0.315800399 & 0.079874180 \\ 0.486582858 & 0.167014172 & 0.083039481 \\ 0.679436682 & 0.014186178 & 0.083336485 \\ 0.833683334 & 0.319990871 & 0.083075089 \\ 0.985047992 & 0.165773890 & 0.082734881 \\ 0.179506512 & 0.513297827 & 0.082902580 \\ 0.332468354 & 0.820611065 & 0.082989030 \\ 0.484575256 & 0.670380829 & 0.082584278 \\ 0.683156105 & 0.513862103 & 0.082966710 \\ 0.832595762 & 0.819579180 & 0.083382452 \\ 0.986555787 & 0.666337587 & 0.083067404 \\ 0.150722901 & 0.152996311 & 0.250126275 \\ -0.000240536 & 0.346870055 & 0.250001666 \\ 0.348331394 & -0.002554859 & 0.250354303 \\ 0.653790326 & 0.153413443 & 0.250266727 \\ 0.503711390 & 0.348820620 & 0.253574221 \\ 0.846911422 & 0.000620575 & 0.250151722 \\ 0.153897135 & 0.652789899 & 0.249811807\end{array}$




\begin{tabular}{|c|c|c|}
\hline 0.000613055 & 0.847247525 & 0.249801436 \\
\hline 0.346777633 & 0.499934107 & 0.250176703 \\
\hline 0.657793428 & 0.657431249 & 0.252461420 \\
\hline 0.500665348 & 0.846557976 & 0.250274180 \\
\hline 0.848066083 & 0.500832812 & 0.250389749 \\
\hline 0.013775454 & 0.180564297 & 0.417086696 \\
\hline 0.320216130 & 0.334110089 & 0.416773637 \\
\hline 0.166715947 & 0.486805927 & 0.416970519 \\
\hline 0.514262625 & 0.179976485 & 0.417689732 \\
\hline 0.820857204 & 0.334053224 & 0.417240330 \\
\hline 0.667724521 & 0.487044254 & 0.417922391 \\
\hline 0.014021717 & 0.680593951 & 0.416769331 \\
\hline 0.319864344 & 0.833125740 & 0.417174680 \\
\hline 0.167338331 & 0.986901990 & 0.416436659 \\
\hline 0.514120937 & 0.680511851 & 0.417105586 \\
\hline 0.821324399 & 0.835029416 & 0.417801185 \\
\hline 0.666795417 & 0.986940081 & 0.417203057 \\
\hline 0.486484203 & 0.319909781 & 0.583468091 \\
\hline 0.180289376 & 0.166727412 & 0.583148208 \\
\hline 0.333365283 & 0.013642467 & 0.583257265 \\
\hline 0.986713605 & 0.319917039 & 0.583418268 \\
\hline 0.680392228 & 0.166975289 & 0.583591244 \\
\hline 0.833254692 & 0.013745354 & 0.583524331 \\
\hline 0.486589893 & 0.819808664 & 0.583489326 \\
\hline 0.180376643 & 0.666825296 & 0.583366918 \\
\hline 0.333331395 & 0.513571764 & 0.583454198 \\
\hline 0.986913156 & 0.819951724 & 0.583405238 \\
\hline 0.680413503 & 0.666967006 & 0.583665972 \\
\hline 0.833586793 & 0.513869097 & 0.583670279 \\
\hline 0.346724900 & 0.346747437 & 0.749642795 \\
\hline 0.152804437 & -0.000359957 & 0.749441614 \\
\hline-0.000309708 & 0.152833250 & 0.749419927 \\
\hline 0.846586021 & 0.346823289 & 0.749685029 \\
\hline 0.652840531 & -0.000143086 & 0.749736517 \\
\hline 0.499884774 & 0.153127894 & 0.749656393 \\
\hline 0.346645360 & 0.846873620 & 0.749636957 \\
\hline 0.152869084 & 0.499785959 & 0.749607139 \\
\hline-0.000048395 & 0.652969861 & 0.749650584 \\
\hline 0.846306936 & 0.846703940 & 0.749729958 \\
\hline 0.652927651 & 0.499955445 & 0.749940167 \\
\hline 0.499836410 & 0.653013021 & 0.749874221 \\
\hline 0.319032409 & 0.486615245 & 0.915651243 \\
\hline 0.165489753 & 0.179607179 & 0.915040584 \\
\hline 0.012409216 & 0.332635737 & 0.915858235 \\
\hline
\end{tabular}




$\begin{array}{lll}0.819541048 & 0.486262197 & 0.916001057 \\ 0.666603165 & 0.180057332 & 0.916112184 \\ 0.513084007 & 0.332733762 & 0.916375467 \\ 0.319110864 & 0.986234678 & 0.916001856 \\ 0.166371970 & 0.679860095 & 0.916018426 \\ 0.012021117 & 0.831921212 & 0.915496954 \\ 0.819210468 & 0.986228518 & 0.916282539 \\ 0.665891770 & 0.679869317 & 0.916697784 \\ 0.513362088 & 0.833753832 & 0.915848329 \\ 0.165767464 & 0.333004411 & 0.020166609 \\ 0.666363680 & 0.333197706 & 0.020844358 \\ 0.165859706 & 0.832546818 & 0.020292955 \\ 0.665230663 & 0.832957642 & 0.021680463 \\ 0.001297260 & 0.000157129 & 0.145498344 \\ 0.497750867 & 0.000266083 & 0.146507583 \\ 0.000424834 & 0.500639759 & 0.146031267 \\ 0.499245134 & 0.495708496 & 0.145505177 \\ 0.334368354 & 0.171338510 & 0.187353347 \\ 0.832988743 & 0.166285348 & 0.187068877 \\ 0.335768023 & 0.666592152 & 0.186566770 \\ 0.831763666 & 0.666510203 & 0.187682230 \\ 0.167153434 & 0.333799103 & 0.312268326 \\ 0.667521244 & 0.333608903 & 0.313054283 \\ 0.167917978 & 0.833857552 & 0.311436745 \\ 0.667407598 & 0.834296027 & 0.312941741 \\ 0.000748830 & 0.000802647 & 0.353311616 \\ 0.499571759 & -0.000130019 & 0.353576467 \\ 0.000584717 & 0.500675755 & 0.353569016 \\ 0.502068884 & 0.500758526 & 0.352203762 \\ 0.333787208 & 0.167361146 & 0.478668831 \\ 0.833526698 & 0.166925322 & 0.479284747 \\ 0.333909754 & 0.666642626 & 0.479137125 \\ 0.834093667 & 0.667495921 & 0.479315296 \\ 0.166916067 & 0.333506703 & 0.520117714 \\ 0.666927809 & 0.333844947 & 0.520596996 \\ 0.167102349 & 0.833538574 & 0.520005143 \\ 0.666859987 & 0.833468069 & 0.520373813 \\ -0.000023721 & 0.000052468 & 0.645834454 \\ 0.499987509 & 0.000042939 & 0.645922739 \\ 0.000211492 & 0.500100815 & 0.645971878 \\ 0.500040909 & 0.500138887 & 0.646164499 \\ 0.333140242 & 0.166494123 & 0.686970345 \\ 0.833053891 & 0.166593340 & 0.687175194 \\ 0.333229973 & 0.666653703 & 0.687205478\end{array}$




$\begin{array}{rcc}0.833222480 & 0.666623106 & 0.687288440 \\ 0.166292806 & 0.332843255 & 0.812558820 \\ 0.666397286 & 0.333273122 & 0.812945676 \\ 0.166378767 & 0.833219818 & 0.812825916 \\ 0.666097924 & 0.833178263 & 0.813134553 \\ -0.000950719 & -0.000850092 & 0.853755056 \\ 0.499372058 & 0.000148507 & 0.853956646 \\ -0.000198761 & 0.499834191 & 0.853891340 \\ 0.499450589 & 0.499438127 & 0.854484570 \\ 0.331195873 & 0.166041290 & 0.980561503 \\ 0.832681808 & 0.166096419 & 0.979512332 \\ 0.333766795 & 0.666960607 & 0.979614014 \\ 0.832480856 & 0.665961960 & 0.979881298\end{array}$

Interpolating coordinate: $0.450(-823.099 \mathrm{eV})$

$\begin{array}{ccc}0.175903434 & 0.009552610 & 0.081079614 \\ 0.328355150 & 0.316075607 & 0.079828967 \\ 0.486578113 & 0.167005168 & 0.083024011 \\ 0.679426381 & 0.014168311 & 0.083334700 \\ 0.833665706 & 0.319989082 & 0.083065890 \\ 0.985061886 & 0.165795361 & 0.082724780 \\ 0.179505133 & 0.513312249 & 0.082892981 \\ 0.332482847 & 0.820573988 & 0.082976268 \\ 0.484617922 & 0.670258977 & 0.082608465 \\ 0.683070382 & 0.513850858 & 0.082961540 \\ 0.832597560 & 0.819577978 & 0.083367743 \\ 0.986524909 & 0.666320677 & 0.083054838 \\ 0.150639962 & 0.152985985 & 0.250121956 \\ -0.000255025 & 0.346868815 & 0.249993270 \\ 0.348377454 & -0.002674614 & 0.250378526 \\ 0.653793722 & 0.153430690 & 0.250255940 \\ 0.503886883 & 0.349098476 & 0.253527069 \\ 0.846884098 & 0.000603930 & 0.250138260 \\ 0.153888772 & 0.652772712 & 0.249811133 \\ 0.000618712 & 0.847247646 & 0.249787825 \\ 0.346778949 & 0.499928733 & 0.250162443 \\ 0.657735984 & 0.657420301 & 0.252394187 \\ 0.500682367 & 0.846522042 & 0.250261305 \\ 0.848082286 & 0.500857453 & 0.250379299 \\ 0.013769873 & 0.180564701 & 0.417086230 \\ 0.320214601 & 0.334101889 & 0.416764053 \\ 0.166717165 & 0.486802968 & 0.416965036 \\ 0.514262228 & 0.180006617 & 0.417661030 \\ 0.820857534 & 0.334059757 & 0.417235631\end{array}$




\begin{tabular}{|c|c|c|}
\hline 0.667743305 & 0.487066990 & 0.417930508 \\
\hline 0.014026378 & 0.680590281 & 0.416772515 \\
\hline 0.319866013 & 0.833114923 & 0.417190812 \\
\hline 0.167339915 & 0.986900299 & 0.416427340 \\
\hline 0.514124540 & 0.680514664 & 0.417106496 \\
\hline 0.821317743 & 0.835014407 & 0.417780660 \\
\hline 0.666799896 & 0.986939251 & 0.417195525 \\
\hline 0.486486249 & 0.319910573 & 0.583461568 \\
\hline 0.180288187 & 0.166725636 & 0.583144363 \\
\hline 0.333371124 & 0.013646667 & 0.583251268 \\
\hline 0.986711366 & 0.319916384 & 0.583416904 \\
\hline 0.680387119 & 0.166975840 & 0.583584159 \\
\hline 0.833255766 & 0.013746813 & 0.583520511 \\
\hline 0.486596155 & 0.819809344 & 0.583491151 \\
\hline 0.180379492 & 0.666824702 & 0.583370958 \\
\hline 0.333328395 & 0.513565692 & 0.583450011 \\
\hline 0.986920057 & 0.819957099 & 0.583403580 \\
\hline 0.680419124 & 0.666974562 & 0.583664296 \\
\hline 0.833587900 & 0.513870771 & 0.583672301 \\
\hline 0.346722059 & 0.346749028 & 0.749635880 \\
\hline 0.152806629 & -0.000352910 & 0.749440492 \\
\hline-0.000311771 & 0.152833993 & 0.749420757 \\
\hline 0.846578919 & 0.346819843 & 0.749683386 \\
\hline 0.652838464 & -0.000144840 & 0.749737604 \\
\hline 0.499878057 & 0.153120255 & 0.749652388 \\
\hline 0.346644984 & 0.846872346 & 0.749639466 \\
\hline 0.152861422 & 0.499786616 & 0.749601664 \\
\hline-0.000052412 & 0.652969368 & 0.749648217 \\
\hline 0.846308114 & 0.846708706 & 0.749726964 \\
\hline 0.652921896 & 0.499953270 & 0.749935554 \\
\hline 0.499838405 & 0.653016006 & 0.749867850 \\
\hline 0.319025512 & 0.486642672 & 0.915623790 \\
\hline 0.165501970 & 0.179628029 & 0.915049203 \\
\hline 0.012402387 & 0.332640010 & 0.915853766 \\
\hline 0.819528078 & 0.486261216 & 0.916000372 \\
\hline 0.666599146 & 0.180052558 & 0.916105508 \\
\hline 0.513075984 & 0.332723466 & 0.916366797 \\
\hline 0.319107745 & 0.986235744 & 0.916003143 \\
\hline 0.166368576 & 0.679856380 & 0.916012627 \\
\hline 0.012006328 & 0.831905017 & 0.915476596 \\
\hline 0.819206446 & 0.986223332 & 0.916282825 \\
\hline 0.665886800 & 0.679866077 & 0.916687650 \\
\hline 0.513356335 & 0.833739533 & 0.915862747 \\
\hline 0.165763854 & 0.333026684 & 0.020148371 \\
\hline
\end{tabular}




\begin{tabular}{|c|c|c|}
\hline 0.666335329 & 0.333178650 & 0.020835142 \\
\hline 0.165854266 & 0.832544094 & 0.020284617 \\
\hline 0.665239067 & 0.832957311 & 0.021679175 \\
\hline 0.001319249 & 0.000178488 & 0.145484106 \\
\hline 0.497725501 & 0.000276268 & 0.146516963 \\
\hline 0.000406691 & 0.500624197 & 0.146027815 \\
\hline 0.499198108 & 0.495683329 & 0.145530729 \\
\hline 0.334323864 & 0.171315451 & 0.187379023 \\
\hline 0.832972863 & 0.166270414 & 0.187064736 \\
\hline 0.335743217 & 0.666602177 & 0.186576381 \\
\hline 0.831787212 & 0.666533560 & 0.187668335 \\
\hline 0.167130610 & 0.333782236 & 0.312259475 \\
\hline 0.667521612 & 0.333633319 & 0.313034603 \\
\hline 0.167933415 & 0.833859501 & 0.311436322 \\
\hline 0.667406765 & 0.834295438 & 0.312932590 \\
\hline 0.000752561 & 0.000810741 & 0.353296366 \\
\hline 0.499557094 & -0.000138598 & 0.353571385 \\
\hline 0.000583070 & 0.500676638 & 0.353569336 \\
\hline 0.502077189 & 0.500759472 & 0.352241021 \\
\hline 0.333786980 & 0.167355257 & 0.478658986 \\
\hline 0.833519631 & 0.166925258 & 0.479277551 \\
\hline 0.333907936 & 0.666634732 & 0.479140424 \\
\hline 0.834112063 & 0.667512012 & 0.479317369 \\
\hline 0.166908378 & 0.333499674 & 0.520112623 \\
\hline 0.666933202 & 0.333853125 & 0.520587889 \\
\hline 0.167110335 & 0.833537479 & 0.520001717 \\
\hline 0.666863392 & 0.833474409 & 0.520365881 \\
\hline-0.000025009 & 0.000052862 & 0.645831072 \\
\hline 0.499989375 & 0.000043576 & 0.645919870 \\
\hline 0.000210749 & 0.500100733 & 0.645969719 \\
\hline 0.500043662 & 0.500141744 & 0.646156397 \\
\hline 0.333140423 & 0.166497131 & 0.686962782 \\
\hline 0.833051866 & 0.166592798 & 0.687171424 \\
\hline 0.333229364 & 0.666655071 & 0.687203488 \\
\hline 0.833219072 & 0.666623897 & 0.687285628 \\
\hline 0.166293396 & 0.332851075 & 0.812550715 \\
\hline 0.666382403 & 0.333263808 & 0.812940068 \\
\hline 0.166374623 & 0.833223587 & 0.812819982 \\
\hline 0.666098585 & 0.833175127 & 0.813129191 \\
\hline-0.000939594 & -0.000833702 & 0.853755883 \\
\hline 0.499362269 & 0.000138365 & 0.853959042 \\
\hline-0.000213674 & 0.499831231 & 0.853885361 \\
\hline 0.499443163 & 0.499429304 & 0.854474423 \\
\hline 0.331196934 & 0.166039252 & 0.980599361 \\
\hline
\end{tabular}




$\begin{array}{lll}0.832673723 & 0.166095550 & 0.979511563 \\ 0.333746027 & 0.666949572 & 0.979608706 \\ 0.832476536 & 0.665968789 & 0.979865382\end{array}$

Interpolating coordinate: $0.467(-823.052 \mathrm{eV})$

$\begin{array}{lll}0.175843203 & 0.009540246 & 0.081013093 \\ 0.328525134 & 0.316350815 & 0.079783754 \\ 0.486573367 & 0.166996165 & 0.083008541 \\ 0.679416081 & 0.014150443 & 0.083332916 \\ 0.833648078 & 0.319987293 & 0.083056691 \\ 0.985075780 & 0.165816832 & 0.082714678 \\ 0.179503754 & 0.513326671 & 0.082883382 \\ 0.332497339 & 0.820536910 & 0.082963506 \\ 0.484660588 & 0.670137124 & 0.082632652 \\ 0.682984660 & 0.513839613 & 0.082956370 \\ 0.832599357 & 0.819576776 & 0.083353035 \\ 0.986494031 & 0.666303767 & 0.083042273 \\ 0.150557024 & 0.152975660 & 0.250117638 \\ -0.000269514 & 0.346867576 & 0.249984873 \\ 0.348423513 & -0.002794368 & 0.250402749 \\ 0.653797119 & 0.153447937 & 0.250245154 \\ 0.504062375 & 0.349376331 & 0.253479917 \\ 0.846856774 & 0.000587284 & 0.250124798 \\ 0.153880410 & 0.652755525 & 0.249810458 \\ 0.000624369 & 0.847247767 & 0.249774215 \\ 0.346780266 & 0.499923359 & 0.250148182 \\ 0.657678540 & 0.657409353 & 0.252326953 \\ 0.500699386 & 0.846486108 & 0.250248429 \\ 0.848098490 & 0.500882093 & 0.250368849 \\ 0.013764292 & 0.180565106 & 0.417085763 \\ 0.320213071 & 0.334093689 & 0.416754469 \\ 0.166718382 & 0.486800009 & 0.416959554 \\ 0.514261831 & 0.180036750 & 0.417632327 \\ 0.820857863 & 0.334066291 & 0.417230933 \\ 0.667762089 & 0.487089727 & 0.417938626 \\ 0.014031039 & 0.680586611 & 0.416775700 \\ 0.319867681 & 0.833104106 & 0.417206944 \\ 0.167341498 & 0.986898608 & 0.416418021 \\ 0.514128143 & 0.680517477 & 0.417107407 \\ 0.821311086 & 0.834999397 & 0.417760135 \\ 0.666804375 & 0.986938422 & 0.417187993 \\ 0.486488295 & 0.319911364 & 0.583455045 \\ 0.180286998 & 0.166723860 & 0.583140519 \\ 0.333376966 & 0.013650867 & 0.583245270\end{array}$




\begin{tabular}{|c|c|c|}
\hline 0.986709126 & 0.319915728 & 0.583415541 \\
\hline 0.680382009 & 0.166976391 & 0.583577074 \\
\hline 0.833256840 & 0.013748273 & 0.583516690 \\
\hline 0.486602418 & 0.819810024 & 0.583492975 \\
\hline 0.180382340 & 0.666824109 & 0.583374997 \\
\hline 0.333325396 & 0.513559620 & 0.583445824 \\
\hline 0.986926958 & 0.819962474 & 0.583401922 \\
\hline 0.680424746 & 0.666982118 & 0.583662620 \\
\hline 0.833589007 & 0.513872445 & 0.583674322 \\
\hline 0.346719217 & 0.346750618 & 0.749628965 \\
\hline 0.152808820 & -0.000345863 & 0.749439369 \\
\hline-0.000313833 & 0.152834736 & 0.749421588 \\
\hline 0.846571817 & 0.346816396 & 0.749681743 \\
\hline 0.652836398 & -0.000146593 & 0.749738691 \\
\hline 0.499871339 & 0.153112617 & 0.749648384 \\
\hline 0.346644609 & 0.846871073 & 0.749641976 \\
\hline 0.152853759 & 0.499787272 & 0.749596188 \\
\hline-0.000056429 & 0.652968875 & 0.749645850 \\
\hline 0.846309292 & 0.846713473 & 0.749723970 \\
\hline 0.652916140 & 0.499951095 & 0.749930941 \\
\hline 0.499840400 & 0.653018991 & 0.749861480 \\
\hline 0.319018616 & 0.486670098 & 0.915596336 \\
\hline 0.165514188 & 0.179648879 & 0.915057821 \\
\hline 0.012395559 & 0.332644283 & 0.915849296 \\
\hline 0.819515108 & 0.486260235 & 0.915999688 \\
\hline 0.666595127 & 0.180047785 & 0.916098833 \\
\hline 0.513067960 & 0.332713171 & 0.916358127 \\
\hline 0.319104627 & 0.986236810 & 0.916004430 \\
\hline 0.166365182 & 0.679852665 & 0.916006828 \\
\hline 0.011991538 & 0.831888821 & 0.915456237 \\
\hline 0.819202424 & 0.986218146 & 0.916283111 \\
\hline 0.665881830 & 0.679862838 & 0.916677516 \\
\hline 0.513350581 & 0.833725234 & 0.915877164 \\
\hline 0.165760243 & 0.333048957 & 0.020130132 \\
\hline 0.666306977 & 0.333159594 & 0.020825925 \\
\hline 0.165848826 & 0.832541370 & 0.020276279 \\
\hline 0.665247472 & 0.832956980 & 0.021677886 \\
\hline 0.001341239 & 0.000199848 & 0.145469868 \\
\hline 0.497700136 & 0.000286452 & 0.146526343 \\
\hline 0.000388548 & 0.500608636 & 0.146024363 \\
\hline 0.499151082 & 0.495658162 & 0.145556282 \\
\hline 0.334279374 & 0.171292392 & 0.187404699 \\
\hline 0.832956982 & 0.166255479 & 0.187060595 \\
\hline 0.335718411 & 0.666612202 & 0.186585992 \\
\hline
\end{tabular}




$\begin{array}{rrr}0.831810757 & 0.666556917 & 0.187654440 \\ 0.167107786 & 0.333765370 & 0.312250625 \\ 0.667521980 & 0.333657735 & 0.313014924 \\ 0.167948852 & 0.833861449 & 0.311435899 \\ 0.667405932 & 0.834294848 & 0.312923440 \\ 0.000756292 & 0.000818835 & 0.353281117 \\ 0.499542429 & -0.000147177 & 0.353566302 \\ 0.000581423 & 0.500677521 & 0.353569657 \\ 0.502085494 & 0.500760418 & 0.352278279 \\ 0.333786752 & 0.167349368 & 0.478649142 \\ 0.833512564 & 0.166925194 & 0.479270356 \\ 0.333906118 & 0.666626838 & 0.479143724 \\ 0.834130459 & 0.667528103 & 0.479319442 \\ 0.166900689 & 0.333492645 & 0.520107532 \\ 0.666938594 & 0.333861304 & 0.520578782 \\ 0.167118320 & 0.833536384 & 0.519998290 \\ 0.666866798 & 0.833480749 & 0.520357949 \\ -0.000026296 & 0.000053256 & 0.645827690 \\ 0.499991241 & 0.000044213 & 0.645917001 \\ 0.000210006 & 0.500100651 & 0.645967560 \\ 0.500046414 & 0.500144602 & 0.646148295 \\ 0.333140605 & 0.166500138 & 0.686955220 \\ 0.833049841 & 0.166592256 & 0.687167655 \\ 0.333228755 & 0.666656439 & 0.687201499 \\ 0.833215664 & 0.666624687 & 0.687282816 \\ 0.166293986 & 0.332858895 & 0.812542610 \\ 0.666367521 & 0.333254495 & 0.812934461 \\ 0.166370478 & 0.833227357 & 0.812814047 \\ 0.666099246 & 0.833171990 & 0.813123830 \\ -0.000928469 & -0.000817312 & 0.853756711 \\ 0.499352481 & 0.000128223 & 0.853961438 \\ -0.000228586 & 0.499828270 & 0.853879381 \\ 0.499435737 & 0.499420481 & 0.854464275 \\ 0.331197994 & 0.166037213 & 0.980637220 \\ 0.832665639 & 0.166094682 & 0.979510794 \\ 0.333725260 & 0.666938537 & 0.979603399 \\ 0.832472216 & 0.665975618 & 0.979849465\end{array}$

Interpolating coordinate: $0.483(-823.060 \mathrm{eV})$

$\begin{array}{lll}0.175782973 & 0.009527881 & 0.080946572 \\ 0.328695118 & 0.316626023 & 0.079738540 \\ 0.486568622 & 0.166987161 & 0.082993071 \\ 0.679405780 & 0.014132576 & 0.083331131 \\ 0.833630449 & 0.319985504 & 0.083047491\end{array}$




$\begin{array}{lcl}0.985089674 & 0.165838303 & 0.082704577 \\ 0.179502374 & 0.513341093 & 0.082873782 \\ 0.332511832 & 0.820499833 & 0.082950744 \\ 0.484703255 & 0.670015272 & 0.082656839 \\ 0.682898938 & 0.513828368 & 0.082951199 \\ 0.832601155 & 0.819575574 & 0.083338326 \\ 0.986463153 & 0.666286857 & 0.083029707 \\ 0.150474085 & 0.152965334 & 0.250113319 \\ -0.000284003 & 0.346866336 & 0.249976477 \\ 0.348469573 & -0.002914123 & 0.250426973 \\ 0.653800515 & 0.153465184 & 0.250234367 \\ 0.504237868 & 0.349654187 & 0.253432764 \\ 0.846829449 & 0.000570639 & 0.250111336 \\ 0.153872047 & 0.652738338 & 0.249809784 \\ 0.000630026 & 0.847247889 & 0.249760604 \\ 0.346781582 & 0.499917985 & 0.250133922 \\ 0.657621095 & 0.657398405 & 0.252259719 \\ 0.500716405 & 0.846450173 & 0.250235554 \\ 0.848114693 & 0.500906734 & 0.250358399 \\ 0.013758711 & 0.180565510 & 0.417085297 \\ 0.320211541 & 0.334085490 & 0.416744886 \\ 0.166719600 & 0.486797049 & 0.416954071 \\ 0.514261435 & 0.180066882 & 0.417603625 \\ 0.820858193 & 0.334072824 & 0.417226235 \\ 0.667780872 & 0.487112463 & 0.417946743 \\ 0.014035699 & 0.680582941 & 0.416778884 \\ 0.319869350 & 0.833093289 & 0.417223075 \\ 0.167343082 & 0.986896917 & 0.416408701 \\ 0.514131746 & 0.680520289 & 0.417108317 \\ 0.821304429 & 0.834984388 & 0.417739610 \\ 0.666808854 & 0.986937592 & 0.417180462 \\ 0.486490341 & 0.319912156 & 0.583448521 \\ 0.180285808 & 0.166722084 & 0.583136675 \\ 0.333382807 & 0.013655067 & 0.583239273 \\ 0.986706887 & 0.319915073 & 0.583414177 \\ 0.680376900 & 0.166976943 & 0.583569989 \\ 0.833257914 & 0.013749733 & 0.583512870 \\ 0.486608680 & 0.819810703 & 0.583494800 \\ 0.180385189 & 0.666823515 & 0.583379037 \\ 0.333322396 & 0.513553547 & 0.583441638 \\ 0.986933858 & 0.819967849 & 0.583400263 \\ 0.680430367 & 0.666989674 & 0.583660944 \\ 0.833590114 & 0.513874119 & 0.583676344 \\ 0.346716376 & 0.346752209 & 0.749622049\end{array}$




$\begin{array}{rrr}0.152811012 & -0.000338817 & 0.749438247 \\ -0.000315896 & 0.152835479 & 0.749422418 \\ 0.846564715 & 0.346812950 & 0.749680099 \\ 0.652834331 & -0.000148347 & 0.749739778 \\ 0.499864622 & 0.153104978 & 0.749644379 \\ 0.346644233 & 0.846869799 & 0.749644485 \\ 0.152846097 & 0.499787929 & 0.749590712 \\ -0.000060447 & 0.652968383 & 0.749643483 \\ 0.846310469 & 0.846718239 & 0.749720976 \\ 0.652910385 & 0.499948920 & 0.749926328 \\ 0.499842396 & 0.653021976 & 0.749855109 \\ 0.319011719 & 0.486697525 & 0.915568883 \\ 0.165526405 & 0.179669730 & 0.915066440 \\ 0.012388730 & 0.332648557 & 0.915844827 \\ 0.819502138 & 0.486259254 & 0.915999003 \\ 0.666591108 & 0.180043011 & 0.916092157 \\ 0.513059937 & 0.332702875 & 0.916349457 \\ 0.319101509 & 0.986237875 & 0.916005717 \\ 0.166361788 & 0.679848949 & 0.916001028 \\ 0.011976749 & 0.831872626 & 0.915435879 \\ 0.819198402 & 0.986212961 & 0.916283396 \\ 0.665876861 & 0.679859598 & 0.916667382 \\ 0.513344828 & 0.833710935 & 0.915891582 \\ 0.165756633 & 0.333071229 & 0.020111894 \\ 0.666278626 & 0.333140538 & 0.020816709 \\ 0.165843386 & 0.832538645 & 0.020267941 \\ 0.665255876 & 0.832956649 & 0.021676597 \\ 0.001363228 & 0.000221207 & 0.145455630 \\ 0.497674770 & 0.000296637 & 0.146535722 \\ 0.000370405 & 0.500593074 & 0.146020911 \\ 0.499104055 & 0.495632995 & 0.145581834 \\ 0.334234884 & 0.171269334 & 0.187430374 \\ 0.832941102 & 0.166240545 & 0.187056454 \\ 0.335693605 & 0.666622227 & 0.186595602 \\ 0.831834302 & 0.666580273 & 0.187640546 \\ 0.167084963 & 0.333748503 & 0.312241774 \\ 0.667522348 & 0.333682151 & 0.312995244 \\ 0.167964289 & 0.833863398 & 0.311435476 \\ 0.667405099 & 0.834294259 & 0.312914289 \\ 0.000760023 & 0.000826928 & 0.353265867 \\ 0.499527763 & -0.000155755 & 0.353561220 \\ 0.000579776 & 0.500678403 & 0.353569977 \\ 0.502093799 & 0.500761364 & 0.352315538 \\ 0.333786525 & 0.167343479 & 0.478639298\end{array}$




$\begin{array}{rrc}0.833505497 & 0.166925129 & 0.479263160 \\ 0.333904300 & 0.666618944 & 0.479147023 \\ 0.834148855 & 0.667544194 & 0.479321515 \\ 0.166893001 & 0.333485617 & 0.520102441 \\ 0.666943987 & 0.333869482 & 0.520569675 \\ 0.167126306 & 0.833535289 & 0.519994864 \\ 0.666870203 & 0.833487089 & 0.520350017 \\ -0.000027584 & 0.000053650 & 0.645824308 \\ 0.499993106 & 0.000044850 & 0.645914132 \\ 0.000209264 & 0.500100569 & 0.645965401 \\ 0.500049167 & 0.500147459 & 0.646140193 \\ 0.333140786 & 0.166503146 & 0.686947657 \\ 0.833047816 & 0.166591714 & 0.687163885 \\ 0.333228146 & 0.666657807 & 0.687199509 \\ 0.833212256 & 0.666625478 & 0.687280004 \\ 0.166294577 & 0.332866715 & 0.812534504 \\ 0.666352639 & 0.333245181 & 0.812928853 \\ 0.166366334 & 0.833231126 & 0.812808113 \\ 0.666099907 & 0.833168854 & 0.813118468 \\ -0.000917344 & -0.000800922 & 0.853757538 \\ 0.499342692 & 0.000118080 & 0.853963834 \\ -0.000243499 & 0.499825310 & 0.853873402 \\ 0.499428311 & 0.499411657 & 0.854454127 \\ 0.331199055 & 0.166035175 & 0.980675078 \\ 0.832657554 & 0.166093813 & 0.979510026 \\ 0.333704492 & 0.666927502 & 0.979598091 \\ 0.832467896 & 0.665982448 & 0.979833549\end{array}$

Interpolating coordinate: $0.500(-823.055 \mathrm{eV})$

$\begin{array}{rrr}0.175722742 & 0.009515516 & 0.080880051 \\ 0.328865102 & 0.316901231 & 0.079693327 \\ 0.486563877 & 0.166978158 & 0.082977601 \\ 0.679395480 & 0.014114708 & 0.083329346 \\ 0.833612821 & 0.319983715 & 0.083038292 \\ 0.985103568 & 0.165859774 & 0.082694475 \\ 0.179500995 & 0.513355515 & 0.082864183 \\ 0.332526324 & 0.820462755 & 0.082937982 \\ 0.484745921 & 0.669893420 & 0.082681026 \\ 0.682813215 & 0.513817123 & 0.082946029 \\ 0.832602952 & 0.819574372 & 0.083323617 \\ 0.986432275 & 0.666269947 & 0.083017141 \\ 0.150391147 & 0.152955009 & 0.250109001 \\ -0.000298492 & 0.346865096 & 0.249968080 \\ 0.348515632 & -0.003033878 & 0.250451196\end{array}$




\begin{tabular}{|c|c|c|}
\hline 0.653803911 & 0.153482431 & 0.250223581 \\
\hline 0.504413361 & 0.349932043 & 0.253385612 \\
\hline 0.846802125 & 0.000553993 & 0.250097874 \\
\hline 0.153863685 & 0.652721151 & 0.249809110 \\
\hline 0.000635683 & 0.847248010 & 0.249746994 \\
\hline 0.346782899 & 0.499912611 & 0.250119662 \\
\hline 0.657563651 & 0.657387457 & 0.252192486 \\
\hline 0.500733424 & 0.846414239 & 0.250222678 \\
\hline 0.848130897 & 0.500931374 & 0.250347949 \\
\hline 0.013753130 & 0.180565915 & 0.417084831 \\
\hline 0.320210012 & 0.334077290 & 0.416735302 \\
\hline 0.166720817 & 0.486794090 & 0.416948588 \\
\hline 0.514261038 & 0.180097014 & 0.417574922 \\
\hline 0.820858522 & 0.334079358 & 0.417221536 \\
\hline 0.667799656 & 0.487135199 & 0.417954860 \\
\hline 0.014040360 & 0.680579271 & 0.416782068 \\
\hline 0.319871018 & 0.833082472 & 0.417239207 \\
\hline 0.167344666 & 0.986895226 & 0.416399382 \\
\hline 0.514135349 & 0.680523102 & 0.417109228 \\
\hline 0.821297773 & 0.834969379 & 0.417719085 \\
\hline 0.666813333 & 0.986936762 & 0.417172930 \\
\hline 0.486492387 & 0.319912948 & 0.583441998 \\
\hline 0.180284619 & 0.166720308 & 0.583132830 \\
\hline 0.333388649 & 0.013659267 & 0.583233275 \\
\hline 0.986704648 & 0.319914417 & 0.583412813 \\
\hline 0.680371791 & 0.166977494 & 0.583562904 \\
\hline 0.833258988 & 0.013751192 & 0.583509049 \\
\hline 0.486614942 & 0.819811383 & 0.583496625 \\
\hline 0.180388038 & 0.666822921 & 0.583383076 \\
\hline 0.333319397 & 0.513547475 & 0.583437451 \\
\hline 0.986940759 & 0.819973224 & 0.583398605 \\
\hline 0.680435988 & 0.666997230 & 0.583659268 \\
\hline 0.833591221 & 0.513875793 & 0.583678365 \\
\hline 0.346713535 & 0.346753799 & 0.749615134 \\
\hline 0.152813204 & -0.000331770 & 0.749437124 \\
\hline-0.000317959 & 0.152836222 & 0.749423248 \\
\hline 0.846557613 & 0.346809504 & 0.749678456 \\
\hline 0.652832265 & -0.000150101 & 0.749740865 \\
\hline 0.499857905 & 0.153097339 & 0.749640374 \\
\hline 0.346643858 & 0.846868526 & 0.749646994 \\
\hline 0.152838434 & 0.499788585 & 0.749585237 \\
\hline-0.000064464 & 0.652967890 & 0.749641116 \\
\hline 0.846311647 & 0.846723006 & 0.749717982 \\
\hline 0.652904629 & 0.499946745 & 0.749921715 \\
\hline
\end{tabular}




$\begin{array}{ccc}0.499844391 & 0.653024961 & 0.749848738 \\ 0.319004823 & 0.486724951 & 0.915541430 \\ 0.165538622 & 0.179690580 & 0.915075059 \\ 0.012381902 & 0.332652830 & 0.915840357 \\ 0.819489168 & 0.486258273 & 0.915998319 \\ 0.666587089 & 0.180038238 & 0.916085482 \\ 0.513051914 & 0.332692580 & 0.916340787 \\ 0.319098390 & 0.986238941 & 0.916007004 \\ 0.166358394 & 0.679845234 & 0.915995229 \\ 0.011961959 & 0.831856431 & 0.915415520 \\ 0.819194380 & 0.986207775 & 0.916283682 \\ 0.665871891 & 0.679856358 & 0.916657248 \\ 0.513339075 & 0.833696636 & 0.915905999 \\ 0.165753022 & 0.333093502 & 0.020093655 \\ 0.666250275 & 0.333121482 & 0.020807492 \\ 0.165837946 & 0.832535921 & 0.020259603 \\ 0.665264281 & 0.832956318 & 0.021675309 \\ 0.001385218 & 0.000242567 & 0.145441392 \\ 0.497649405 & 0.000306821 & 0.146545102 \\ 0.000352262 & 0.500577512 & 0.146017459 \\ 0.499057029 & 0.495607828 & 0.145607386 \\ 0.334190394 & 0.171246275 & 0.187456050 \\ 0.832925221 & 0.166225611 & 0.187052313 \\ 0.335668799 & 0.666632252 & 0.186605213 \\ 0.831857848 & 0.666603630 & 0.187626651 \\ 0.167062139 & 0.333731636 & 0.312232923 \\ 0.667522716 & 0.333706567 & 0.312975564 \\ 0.167979726 & 0.833865346 & 0.311435053 \\ 0.667404266 & 0.834293669 & 0.312905139 \\ 0.000763754 & 0.000835022 & 0.353250617 \\ 0.499513098 & -0.000164334 & 0.353556137 \\ 0.000578129 & 0.500679286 & 0.353570298 \\ 0.502102104 & 0.500762310 & 0.352352796 \\ 0.333786297 & 0.167337590 & 0.478629453 \\ 0.833498430 & 0.166925065 & 0.479255964 \\ 0.333902482 & 0.666611050 & 0.479150323 \\ 0.834167251 & 0.667560285 & 0.479323588 \\ 0.166885312 & 0.333478588 & 0.520097350 \\ 0.666949380 & 0.333877661 & 0.520560568 \\ 0.167134292 & 0.833534194 & 0.519991437 \\ 0.666873608 & 0.833493429 & 0.520342085 \\ 0.499909898972 & 0.000054044 & 0.645820926 \\ 0.000208521 & 0.000045487 & 0.645911263 \\ & 0.500100487 & 0.645963242\end{array}$




$\begin{array}{rrr}0.500051919 & 0.500150317 & 0.646132091 \\ 0.333140968 & 0.166506153 & 0.686940095 \\ 0.833045791 & 0.166591172 & 0.687160115 \\ 0.333227537 & 0.6666559175 & 0.687197519 \\ 0.833208848 & 0.666626268 & 0.687277192 \\ 0.166295167 & 0.332874535 & 0.812526399 \\ 0.666337756 & 0.333235868 & 0.812923246 \\ 0.166362189 & 0.833234895 & 0.812802178 \\ 0.666100568 & 0.833165717 & 0.813113107 \\ -0.000906219 & -0.000784532 & 0.853758365 \\ 0.499332904 & 0.000107938 & 0.853966230 \\ -0.000258412 & 0.499822350 & 0.853867423 \\ 0.499420885 & 0.499402834 & 0.854443980 \\ 0.331200115 & 0.166033136 & 0.980712937 \\ 0.832649470 & 0.166092944 & 0.979509257 \\ 0.333683725 & 0.666916467 & 0.979592784 \\ 0.832463576 & 0.665989277 & 0.979817632\end{array}$

Interpolating coordinate: 0.517 (-823.056 eV)

$\begin{array}{lrl}0.175662511 & 0.009503151 & 0.080813530 \\ 0.329035086 & 0.317176439 & 0.079648114 \\ 0.486559132 & 0.166969155 & 0.082962131 \\ 0.679385180 & 0.014096841 & 0.083327561 \\ 0.833595193 & 0.319981926 & 0.083029093 \\ 0.985117462 & 0.165881245 & 0.082684373 \\ 0.179499616 & 0.513369937 & 0.082854584 \\ 0.332540816 & 0.820425677 & 0.082925220 \\ 0.484788587 & 0.669771568 & 0.082705213 \\ 0.682727492 & 0.513805878 & 0.082940859 \\ 0.832604749 & 0.819573170 & 0.083308908 \\ 0.986401397 & 0.666253037 & 0.083004575 \\ 0.150308209 & 0.152944684 & 0.250104683 \\ -0.000312981 & 0.346863856 & 0.249959684 \\ 0.348561691 & -0.003153633 & 0.250475419 \\ 0.653807307 & 0.153499678 & 0.250212795 \\ 0.504588854 & 0.350209899 & 0.253338460 \\ 0.846774801 & 0.000537347 & 0.250084412 \\ 0.153855323 & 0.652703964 & 0.249808436 \\ 0.000641340 & 0.847248131 & 0.249733384 \\ 0.346784216 & 0.499907237 & 0.250105402 \\ 0.657506207 & 0.657376509 & 0.252125253 \\ 0.500750443 & 0.846378305 & 0.250209802 \\ 0.848147101 & 0.500956015 & 0.250337499 \\ 0.013747549 & 0.180566320 & 0.417084365\end{array}$




$\begin{array}{ccc}0.320208483 & 0.334069090 & 0.416725718 \\ 0.166722034 & 0.486791131 & 0.416943105 \\ 0.514260641 & 0.180127146 & 0.417546219 \\ 0.820858851 & 0.334085891 & 0.417216837 \\ 0.667818440 & 0.487157935 & 0.417962977 \\ 0.014045021 & 0.680575601 & 0.416785252 \\ 0.319872687 & 0.833071655 & 0.417255339 \\ 0.167346250 & 0.986893535 & 0.416390063 \\ 0.514138952 & 0.680525915 & 0.417110139 \\ 0.821291116 & 0.834954370 & 0.417698560 \\ 0.666817812 & 0.986935932 & 0.417165398 \\ 0.486494433 & 0.319913740 & 0.583435475 \\ 0.180283430 & 0.166718532 & 0.583128985 \\ 0.333394491 & 0.013663467 & 0.583227278 \\ 0.986702409 & 0.319913761 & 0.583411449 \\ 0.680366682 & 0.166978045 & 0.583555819 \\ 0.833260062 & 0.013752651 & 0.583505228 \\ 0.486621204 & 0.819812063 & 0.583498450 \\ 0.180390887 & 0.666822327 & 0.583387115 \\ 0.333316398 & 0.513541403 & 0.583433264 \\ 0.986947660 & 0.819978599 & 0.583396947 \\ 0.680441609 & 0.667004786 & 0.583657592 \\ 0.833592328 & 0.513877467 & 0.583680386 \\ 0.346710694 & 0.346755389 & 0.749608219 \\ 0.152815396 & -0.000324723 & 0.749436001 \\ -0.000320022 & 0.152836965 & 0.749424078 \\ 0.846550511 & 0.346806058 & 0.749676813 \\ 0.652830199 & -0.000151855 & 0.749741952 \\ 0.499851188 & 0.153089700 & 0.749636369 \\ 0.346643483 & 0.846867252 & 0.749649503 \\ 0.152830771 & 0.499789241 & 0.749579762 \\ -0.000068481 & 0.652967397 & 0.749638749 \\ 0.846312825 & 0.846727773 & 0.749714988 \\ 0.652898873 & 0.499944570 & 0.749917102 \\ 0.499846386 & 0.653027946 & 0.749842367 \\ 0.318997926 & 0.486752377 & 0.915513977 \\ 0.165550839 & 0.179711430 & 0.915083678 \\ 0.012375074 & 0.332657103 & 0.915835887 \\ 0.819476198 & 0.486257292 & 0.915997634 \\ 0.666583070 & 0.180033464 & 0.916078806 \\ 0.513043891 & 0.332682285 & 0.916332117 \\ 0.319095271 & 0.986240007 & 0.916008291 \\ 0.166355000 & 0.679841519 & 0.915989430 \\ 0.011947170 & 0.831840236 & 0.915395161\end{array}$




$\begin{array}{rrr}0.819190358 & 0.986202589 & 0.916283968 \\ 0.665866921 & 0.679853118 & 0.916647114 \\ 0.513333322 & 0.833682337 & 0.915920417 \\ 0.165749411 & 0.333115775 & 0.020075416 \\ 0.666221924 & 0.333102426 & 0.020798276 \\ 0.165832506 & 0.832533197 & 0.020251265 \\ 0.665272686 & 0.832955987 & 0.021674021 \\ 0.001407207 & 0.000263927 & 0.145427154 \\ 0.497624040 & 0.000317005 & 0.146554482 \\ 0.000334119 & 0.500561950 & 0.146014007 \\ 0.499010003 & 0.495582661 & 0.145632938 \\ 0.334145904 & 0.171223216 & 0.187481726 \\ 0.832909340 & 0.166210677 & 0.187048172 \\ 0.335643993 & 0.666642277 & 0.186614824 \\ 0.831881393 & 0.666626987 & 0.187612756 \\ 0.167039315 & 0.333714769 & 0.312224072 \\ 0.667523084 & 0.333730983 & 0.312955884 \\ 0.167995163 & 0.833867294 & 0.311434630 \\ 0.667403433 & 0.834293079 & 0.312895989 \\ 0.000767485 & 0.000843116 & 0.353235367 \\ 0.499498433 & -0.000172913 & 0.353551054 \\ 0.000576482 & 0.500680169 & 0.353570619 \\ 0.502110409 & 0.500763256 & 0.352390055 \\ 0.333786069 & 0.167331701 & 0.478619608 \\ 0.833491363 & 0.166925001 & 0.479248768 \\ 0.333900664 & 0.666603156 & 0.479153622 \\ 0.834185647 & 0.667576376 & 0.479325661 \\ 0.166877623 & 0.333471559 & 0.520092259 \\ 0.666954773 & 0.333885840 & 0.520551461 \\ 0.167142278 & 0.833533099 & 0.519988010 \\ 0.666877013 & 0.833499769 & 0.520334153 \\ -0.000030160 & 0.000054438 & 0.645817544 \\ 0.499996838 & 0.000046124 & 0.645908394 \\ 0.000207778 & 0.500100405 & 0.645961083 \\ 0.500054671 & 0.500153175 & 0.646123989 \\ 0.333141150 & 0.166509160 & 0.686932533 \\ 0.833043766 & 0.166590630 & 0.687156345 \\ 0.333226928 & 0.666660543 & 0.687195529 \\ 0.833205440 & 0.666627059 & 0.687274380 \\ 0.166295757 & 0.332882355 & 0.812518294 \\ 0.666322873 & 0.333226554 & 0.812917639 \\ 0.166358044 & 0.833238664 & 0.812796243 \\ 0.666101229 & 0.833162581 & 0.813107745 \\ -0.000895094 & -0.000768142 & 0.853759192\end{array}$




$\begin{array}{rrr}0.499323116 & 0.000097796 & 0.853968626 \\ -0.000273325 & 0.499819390 & 0.853861444 \\ 0.499413459 & 0.499394011 & 0.854433833 \\ 0.331201176 & 0.166031097 & 0.980750796 \\ 0.832641385 & 0.166092075 & 0.979508488 \\ 0.333662958 & 0.666905432 & 0.979587476 \\ 0.832459256 & 0.665996106 & 0.979801715\end{array}$

Interpolating coordinate: $0.533(-823.053 \mathrm{eV})$

$\begin{array}{rrr}0.175602281 & 0.009490787 & 0.080747009 \\ 0.329205070 & 0.317451647 & 0.079602901 \\ 0.486554387 & 0.166960151 & 0.082946661 \\ 0.679374879 & 0.014078973 & 0.083325776 \\ 0.833577564 & 0.319980137 & 0.083019893 \\ 0.985131356 & 0.165902716 & 0.082674272 \\ 0.179498236 & 0.513384359 & 0.082844984 \\ 0.332555309 & 0.820388600 & 0.082912458 \\ 0.484831254 & 0.669649716 & 0.082729400 \\ 0.682641770 & 0.513794633 & 0.082935689 \\ 0.832606547 & 0.819571968 & 0.083294199 \\ 0.986370519 & 0.666236127 & 0.082992009 \\ 0.150225270 & 0.152934358 & 0.250100364 \\ -0.000327470 & 0.346862616 & 0.249951287 \\ 0.348607751 & -0.003273388 & 0.250499643 \\ 0.653810703 & 0.153516925 & 0.250202008 \\ 0.504764346 & 0.350487755 & 0.253291307 \\ 0.846747476 & 0.000520702 & 0.250070950 \\ 0.153846960 & 0.652686777 & 0.249807762 \\ 0.000646997 & 0.847248253 & 0.249719773 \\ 0.346785532 & 0.499901863 & 0.250091142 \\ 0.657448763 & 0.657365561 & 0.252058019 \\ 0.500767462 & 0.846342370 & 0.250196927 \\ 0.848163304 & 0.500980655 & 0.250327049 \\ 0.013741968 & 0.180566724 & 0.417083898 \\ 0.320206953 & 0.334060891 & 0.416716135 \\ 0.166723252 & 0.486788171 & 0.416937623 \\ 0.514260245 & 0.180157278 & 0.417517517 \\ 0.820859181 & 0.334092425 & 0.417212139 \\ 0.667837223 & 0.487180672 & 0.417971095 \\ 0.014049681 & 0.680571931 & 0.416788436 \\ 0.319874355 & 0.833060838 & 0.417271471 \\ 0.167347834 & 0.986891844 & 0.416380744 \\ 0.514142555 & 0.680528727 & 0.417111049 \\ 0.821284460 & 0.834939361 & 0.417678035\end{array}$




$\begin{array}{rrr}0.666822291 & 0.986935103 & 0.417157867 \\ 0.486496479 & 0.319914532 & 0.583428951 \\ 0.180282240 & 0.166716756 & 0.583125141 \\ 0.333400332 & 0.013667667 & 0.583221280 \\ 0.986700169 & 0.319913106 & 0.583410086 \\ 0.680361573 & 0.166978597 & 0.583548734 \\ 0.833261136 & 0.013754111 & 0.583501408 \\ 0.486627466 & 0.819812743 & 0.583500275 \\ 0.180393736 & 0.666821733 & 0.583391155 \\ 0.333313398 & 0.513535330 & 0.583429078 \\ 0.986954560 & 0.819983974 & 0.583395289 \\ 0.680447230 & 0.667012342 & 0.583655916 \\ 0.833593435 & 0.513879141 & 0.583682408 \\ 0.346707852 & 0.346756980 & 0.749601304 \\ 0.152817588 & -0.000317677 & 0.749434879 \\ -0.000322084 & 0.152837708 & 0.749424908 \\ 0.846543409 & 0.346802612 & 0.749675169 \\ 0.652828132 & -0.000153608 & 0.749743039 \\ 0.499844470 & 0.153082061 & 0.749632364 \\ 0.346643107 & 0.846865979 & 0.749652012 \\ 0.152823109 & 0.499789898 & 0.749574286 \\ -0.000072499 & 0.652966905 & 0.749636382 \\ 0.846314002 & 0.846732539 & 0.749711994 \\ 0.652893118 & 0.499942395 & 0.749912489 \\ 0.499848382 & 0.653030931 & 0.749835996 \\ 0.318991030 & 0.486779804 & 0.915486524 \\ 0.165563057 & 0.179732281 & 0.915092297 \\ 0.012368245 & 0.332661377 & 0.915831418 \\ 0.819463228 & 0.486256311 & 0.915996950 \\ 0.666579051 & 0.180028691 & 0.916072131 \\ 0.513035868 & 0.332671989 & 0.916323447 \\ 0.319092153 & 0.986241072 & 0.916009578 \\ 0.166351606 & 0.679837804 & 0.915983630 \\ 0.011932380 & 0.831824040 & 0.915374803 \\ 0.819186336 & 0.986197404 & 0.916284254 \\ 0.665861952 & 0.679849878 & 0.916636980 \\ 0.513327569 & 0.833668038 & 0.915934834 \\ 0.165745801 & 0.333138047 & 0.020057178 \\ 0.666193573 & 0.333083370 & 0.020789059 \\ 0.165827066 & 0.832530472 & 0.020242927 \\ 0.665281090 & 0.832955656 & 0.021672732 \\ 0.001429197 & 0.000285286 & 0.145412916 \\ 0.497598674 & 0.000327190 & 0.146563862 \\ 0.000315976 & 0.500546388 & 0.146010555\end{array}$




$\begin{array}{rrr}0.498962976 & 0.495557494 & 0.145658491 \\ 0.334101414 & 0.171200157 & 0.187507401 \\ 0.832893460 & 0.166195743 & 0.187044031 \\ 0.335619187 & 0.666652302 & 0.186624434 \\ 0.831904939 & 0.666650343 & 0.187598862 \\ 0.167016492 & 0.333697902 & 0.312215222 \\ 0.667523452 & 0.333755399 & 0.312936204 \\ 0.168010600 & 0.833869243 & 0.311434207 \\ 0.667402600 & 0.834292490 & 0.312886838 \\ 0.000771216 & 0.000851209 & 0.353220117 \\ 0.499483768 & -0.000181492 & 0.353545972 \\ 0.000574835 & 0.500681051 & 0.353570939 \\ 0.502118714 & 0.500764202 & 0.352427313 \\ 0.333785842 & 0.167325812 & 0.478609764 \\ 0.833484296 & 0.166924936 & 0.479241572 \\ 0.333898846 & 0.666595262 & 0.479156922 \\ 0.834204043 & 0.667592467 & 0.479327734 \\ 0.166869935 & 0.333464530 & 0.520087168 \\ 0.666960165 & 0.333894018 & 0.520542354 \\ 0.167150264 & 0.833532004 & 0.519984584 \\ 0.666880419 & 0.833506109 & 0.520326221 \\ -0.000031448 & 0.000054832 & 0.645814162 \\ 0.499998703 & 0.000046761 & 0.645905525 \\ 0.000207036 & 0.500100323 & 0.645958924 \\ 0.500057424 & 0.500156032 & 0.646115887 \\ 0.333141331 & 0.166512168 & 0.686924970 \\ 0.833041741 & 0.166590088 & 0.687152576 \\ 0.333226319 & 0.666661911 & 0.687193540 \\ 0.833202032 & 0.666627849 & 0.687271568 \\ 0.166296348 & 0.332890175 & 0.812510188 \\ 0.666307991 & 0.333217241 & 0.812912031 \\ 0.166353900 & 0.833242433 & 0.812790309 \\ 0.666101890 & 0.833159444 & 0.813102384 \\ -0.000883969 & -0.000751752 & 0.853760019 \\ 0.499313327 & 0.000087653 & 0.853971022 \\ -0.000288238 & 0.499816430 & 0.853855465 \\ 0.499406033 & 0.499385187 & 0.854423685 \\ 0.331202236 & 0.166029059 & 0.980788654 \\ 0.832633301 & 0.166091207 & 0.979507720 \\ 0.333642190 & 0.666894397 & 0.979582169 \\ 0.832454936 & 0.666002936 & 0.979785799\end{array}$

Interpolating coordinate: $0.550(-823.099 \mathrm{eV})$

$\begin{array}{lll}0.175542050 & 0.009478422 & 0.080680489\end{array}$ 


$\begin{array}{rrr}0.329375054 & 0.317726855 & 0.079557687 \\ 0.486549642 & 0.166951148 & 0.082931191 \\ 0.679364579 & 0.014061106 & 0.083323992 \\ 0.833559936 & 0.319978348 & 0.083010694 \\ 0.985145251 & 0.165924187 & 0.082664170 \\ 0.179496857 & 0.513398781 & 0.082835385 \\ 0.332569801 & 0.820351522 & 0.082899696 \\ 0.484873920 & 0.669527863 & 0.082753587 \\ 0.682556047 & 0.513783387 & 0.082930518 \\ 0.832608344 & 0.819570766 & 0.083279491 \\ 0.986339641 & 0.666219217 & 0.082979444 \\ 0.150142332 & 0.152924033 & 0.250096046 \\ -0.000341959 & 0.346861376 & 0.249942891 \\ 0.348653810 & -0.003393142 & 0.250523866 \\ 0.653814099 & 0.153534173 & 0.250191221 \\ 0.504939839 & 0.350765610 & 0.253244155 \\ 0.846720152 & 0.000504056 & 0.250057488 \\ 0.153838598 & 0.652669590 & 0.249807087 \\ 0.000652654 & 0.847248374 & 0.249706163 \\ 0.346786849 & 0.499896489 & 0.250076881 \\ 0.657391318 & 0.657354613 & 0.251990785 \\ 0.500784481 & 0.846306436 & 0.250184052 \\ 0.848179508 & 0.501005295 & 0.250316599 \\ 0.013736387 & 0.180567129 & 0.417083432 \\ 0.320205423 & 0.334052691 & 0.416706551 \\ 0.166724469 & 0.486785212 & 0.416932140 \\ 0.514259848 & 0.180187410 & 0.417488814 \\ 0.820859510 & 0.334098958 & 0.417207440 \\ 0.667856007 & 0.487203408 & 0.417979212 \\ 0.014054342 & 0.680568261 & 0.416791621 \\ 0.319876023 & 0.833050021 & 0.417287602 \\ 0.167349417 & 0.986890153 & 0.416371424 \\ 0.514146158 & 0.680531540 & 0.417111960 \\ 0.821277804 & 0.834924352 & 0.417657510 \\ 0.666826770 & 0.986934273 & 0.417150335 \\ 0.486498525 & 0.319915323 & 0.583422428 \\ 0.180281051 & 0.166714980 & 0.583121296 \\ 0.333406174 & 0.013671867 & 0.583215282 \\ 0.986697930 & 0.319912450 & 0.583408722 \\ 0.680356463 & 0.166979148 & 0.583541649 \\ 0.833262210 & 0.013755570 & 0.583497588 \\ 0.486633728 & 0.819813422 & 0.583502099 \\ 0.180396584 & 0.666821139 & 0.583395194 \\ 0.333310399 & 0.513529258 & 0.583424891 \\ & & \end{array}$




$\begin{array}{rrr}0.986961461 & 0.819989349 & 0.583393630 \\ 0.680452852 & 0.667019898 & 0.583654240 \\ 0.833594541 & 0.513880815 & 0.583684429 \\ 0.346705011 & 0.346758570 & 0.749594388 \\ 0.152819780 & -0.000310630 & 0.749433756 \\ -0.000324147 & 0.152838451 & 0.749425738 \\ 0.846536307 & 0.346799165 & 0.749673526 \\ 0.652826066 & -0.000155362 & 0.749744126 \\ 0.499837753 & 0.153074422 & 0.749628360 \\ 0.346642732 & 0.846864705 & 0.749654521 \\ 0.152815446 & 0.499790554 & 0.749568811 \\ -0.000076516 & 0.652966412 & 0.749634015 \\ 0.846315180 & 0.846737306 & 0.749709000 \\ 0.652887363 & 0.499940221 & 0.749907876 \\ 0.499850377 & 0.653033916 & 0.749829626 \\ 0.318984133 & 0.486807231 & 0.915459071 \\ 0.165575274 & 0.179753131 & 0.915100915 \\ 0.012361416 & 0.332665650 & 0.915826948 \\ 0.819450258 & 0.486255330 & 0.915996265 \\ 0.666575032 & 0.180023917 & 0.916065455 \\ 0.513027844 & 0.332661694 & 0.916314777 \\ 0.319089035 & 0.986242138 & 0.916010865 \\ 0.166348212 & 0.679834088 & 0.915977831 \\ 0.011917591 & 0.831807845 & 0.915354445 \\ 0.819182314 & 0.986192218 & 0.916284539 \\ 0.665856982 & 0.679846639 & 0.916626846 \\ 0.513321816 & 0.833653739 & 0.915949252 \\ 0.165742190 & 0.333160320 & 0.020038939 \\ 0.666165221 & 0.333064315 & 0.020779842 \\ 0.165821626 & 0.832527748 & 0.020234589 \\ 0.665289495 & 0.832955325 & 0.021671443 \\ 0.001451186 & 0.000306645 & 0.145398678 \\ 0.497573309 & 0.000337374 & 0.146573241 \\ 0.000297833 & 0.500530827 & 0.146007103 \\ 0.498915950 & 0.495532327 & 0.145684043 \\ 0.334056924 & 0.171177099 & 0.187533077 \\ 0.832877579 & 0.166180808 & 0.187039890 \\ 0.335594382 & 0.666662328 & 0.186634045 \\ 0.831928484 & 0.666673700 & 0.187584967 \\ 0.166993668 & 0.333681036 & 0.312206371 \\ 0.667523820 & 0.333779815 & 0.312916525 \\ 0.168026037 & 0.833871191 & 0.311433784 \\ 0.667401767 & 0.834291900 & 0.312877688 \\ 0.000774947 & 0.000859303 & 0.353204867\end{array}$




$\begin{array}{rrr}0.499469102 & -0.000190070 & 0.353540889 \\ 0.000573188 & 0.500681934 & 0.353571260 \\ 0.502127019 & 0.500765148 & 0.352464571 \\ 0.333785614 & 0.167319923 & 0.478599919 \\ 0.833477229 & 0.166924872 & 0.479234377 \\ 0.333897028 & 0.666587368 & 0.479160221 \\ 0.834222439 & 0.667608557 & 0.479329807 \\ 0.166862246 & 0.333457502 & 0.520082077 \\ 0.666965558 & 0.333902196 & 0.520533247 \\ 0.167158249 & 0.833530909 & 0.519981157 \\ 0.666883824 & 0.833512449 & 0.520318289 \\ -0.000032735 & 0.000055226 & 0.645810780 \\ 0.500000569 & 0.000047398 & 0.645902656 \\ 0.000206293 & 0.500100240 & 0.645956765 \\ 0.500060176 & 0.500158890 & 0.646107784 \\ 0.333141513 & 0.166515175 & 0.686917408 \\ 0.833039716 & 0.166589546 & 0.687148806 \\ 0.333225710 & 0.666663280 & 0.687191550 \\ 0.833198623 & 0.666628640 & 0.687268756 \\ 0.166296938 & 0.332897995 & 0.812502083 \\ 0.666293109 & 0.333207928 & 0.812906424 \\ 0.166349755 & 0.833246203 & 0.812784374 \\ 0.666102551 & 0.833156307 & 0.813097022 \\ -0.000872844 & -0.000735362 & 0.853760847 \\ 0.499303539 & 0.000077511 & 0.853973418 \\ -0.000303150 & 0.499813469 & 0.853849485 \\ 0.499398607 & 0.499376364 & 0.854413537 \\ 0.331203297 & 0.166027020 & 0.980826513 \\ 0.832625216 & 0.166090338 & 0.979506951 \\ 0.333621423 & 0.6668883362 & 0.979576862 \\ 0.832450616 & 0.666009765 & 0.979769882 \\ & & \end{array}$

Interpolating coordinate: $0.567(-823.107 \mathrm{eV})$

$\begin{array}{lll}0.175481819 & 0.009466057 & 0.080613968 \\ 0.329545038 & 0.318002063 & 0.079512474 \\ 0.486544897 & 0.166942145 & 0.082915721 \\ 0.679354278 & 0.014043238 & 0.083322207 \\ 0.833542308 & 0.319976559 & 0.083001495 \\ 0.985159145 & 0.165945658 & 0.082654069 \\ 0.179495477 & 0.513413203 & 0.082825786 \\ 0.332584294 & 0.820314445 & 0.082886934 \\ 0.484916587 & 0.669406011 & 0.082777774 \\ 0.682470325 & 0.513772142 & 0.082925348 \\ 0.832610142 & 0.819569564 & 0.083264782\end{array}$




$\begin{array}{rrr}0.986308763 & 0.666202307 & 0.082966878 \\ 0.150059394 & 0.152913707 & 0.250091727 \\ -0.000356448 & 0.346860136 & 0.249934494 \\ 0.348699870 & -0.003512897 & 0.250548089 \\ 0.653817495 & 0.153551420 & 0.250180435 \\ 0.505115332 & 0.351043466 & 0.253197003 \\ 0.846692827 & 0.000487411 & 0.250044026 \\ 0.153830235 & 0.652652403 & 0.249806413 \\ 0.000658311 & 0.847248495 & 0.249692553 \\ 0.346788166 & 0.499891115 & 0.250062621 \\ 0.657333874 & 0.657343665 & 0.251923552 \\ 0.500801500 & 0.846270502 & 0.250171176 \\ 0.848195711 & 0.501029936 & 0.250306149 \\ 0.013730806 & 0.180567533 & 0.417082966 \\ 0.320203894 & 0.334044491 & 0.416696968 \\ 0.166725686 & 0.486782252 & 0.416926657 \\ 0.514259451 & 0.180217542 & 0.417460112 \\ 0.820859840 & 0.334105492 & 0.417202742 \\ 0.667874791 & 0.487226144 & 0.417987329 \\ 0.014059003 & 0.680564591 & 0.416794805 \\ 0.319877692 & 0.833039204 & 0.417303734 \\ 0.167351001 & 0.986888462 & 0.416362105 \\ 0.514149761 & 0.680534353 & 0.417112870 \\ 0.821271147 & 0.834909343 & 0.417636985 \\ 0.666831249 & 0.986933443 & 0.417142804 \\ 0.486500571 & 0.319916115 & 0.583415904 \\ 0.180279862 & 0.166713204 & 0.583117452 \\ 0.333412016 & 0.013676067 & 0.583209285 \\ 0.986695691 & 0.319911795 & 0.583407358 \\ 0.680351354 & 0.166979699 & 0.583534564 \\ 0.833263284 & 0.013757030 & 0.583493767 \\ 0.486639990 & 0.819814102 & 0.583503924 \\ 0.180399433 & 0.666820545 & 0.583399233 \\ 0.333307399 & 0.513523186 & 0.583420704 \\ 0.986968361 & 0.819994724 & 0.583391972 \\ 0.680458473 & 0.667027454 & 0.583652564 \\ 0.833595648 & 0.513882489 & 0.583686451 \\ 0.346702170 & 0.346760161 & 0.749587473 \\ 0.152821972 & -0.000303584 & 0.749432633 \\ -0.000326210 & 0.152839194 & 0.749426568 \\ 0.846529205 & 0.346795719 & 0.749671883 \\ 0.652823999 & -0.000157116 & 0.749745213 \\ 0.499831036 & 0.153066783 & 0.749624355 \\ 0.346642356 & 0.846863432 & 0.749657030\end{array}$




$\begin{array}{ccc}0.152807784 & 0.499791211 & 0.749563335 \\ -0.000080533 & 0.652965919 & 0.749631648 \\ 0.846316357 & 0.846742072 & 0.749706006 \\ 0.652881607 & 0.499938046 & 0.749903263 \\ 0.499852372 & 0.653036901 & 0.749823255 \\ 0.318977237 & 0.486834657 & 0.915431618 \\ 0.165587491 & 0.179773982 & 0.915109534 \\ 0.012354588 & 0.332669923 & 0.915822478 \\ 0.819437288 & 0.486254349 & 0.915995581 \\ 0.666571013 & 0.180019144 & 0.916058780 \\ 0.513019821 & 0.332651398 & 0.916306107 \\ 0.319085916 & 0.986243204 & 0.916012152 \\ 0.166344818 & 0.679830373 & 0.915972032 \\ 0.011902801 & 0.831791650 & 0.915334086 \\ 0.819178292 & 0.986187032 & 0.916284825 \\ 0.665852013 & 0.679843399 & 0.916616712 \\ 0.513316063 & 0.833639440 & 0.915963669 \\ 0.165738580 & 0.333182593 & 0.020020700 \\ 0.666136870 & 0.333045259 & 0.020770626 \\ 0.165816186 & 0.832525024 & 0.020226251 \\ 0.665297899 & 0.832954994 & 0.021670155 \\ 0.001473176 & 0.000328005 & 0.145384440 \\ 0.497547944 & 0.000347559 & 0.146582621 \\ 0.000279690 & 0.500515265 & 0.146003651 \\ 0.498868924 & 0.495507160 & 0.145709595 \\ 0.334012434 & 0.171154040 & 0.187558753 \\ 0.832861699 & 0.166165874 & 0.187035749 \\ 0.335569576 & 0.666672353 & 0.186643656 \\ 0.831952030 & 0.666697057 & 0.187571073 \\ 0.166970844 & 0.333664169 & 0.312197520 \\ 0.667524188 & 0.333804231 & 0.312896845 \\ 0.168041474 & 0.833873140 & 0.311433361 \\ 0.667400934 & 0.834291311 & 0.312868537 \\ 0.000778678 & 0.000867396 & 0.353189617 \\ 0.499454437 & -0.000198649 & 0.353535806 \\ 0.000571541 & 0.500682816 & 0.353571580 \\ 0.502135324 & 0.500766094 & 0.352501830 \\ 0.333785387 & 0.167314034 & 0.478590075 \\ 0.833470162 & 0.166924808 & 0.479227181 \\ 0.333895210 & 0.666579474 & 0.479163521 \\ 0.834240835 & 0.667624648 & 0.479331880 \\ 0.1668545557 & 0.333450473 & 0.520076986 \\ 0.666970951 & 0.333910375 & 0.520524140 \\ 0.167166235 & 0.833529814 & 0.519977730\end{array}$




$\begin{array}{rcc}0.666887229 & 0.833518789 & 0.520310357 \\ -0.000034023 & 0.000055620 & 0.645807398 \\ 0.500002434 & 0.000048035 & 0.645899787 \\ 0.000205550 & 0.500100158 & 0.645954606 \\ 0.500062929 & 0.500161748 & 0.646099682 \\ 0.333141694 & 0.166518183 & 0.686909845 \\ 0.833037691 & 0.166589004 & 0.687145036 \\ 0.333225101 & 0.666664648 & 0.687189560 \\ 0.833195215 & 0.666629430 & 0.687265944 \\ 0.166297528 & 0.332905815 & 0.812493977 \\ 0.666278226 & 0.333198614 & 0.812900816 \\ 0.166345610 & 0.833249972 & 0.812778440 \\ 0.666103212 & 0.833153171 & 0.813091661 \\ -0.000861719 & -0.000718972 & 0.853761674 \\ 0.499293750 & 0.000067369 & 0.853975814 \\ -0.000318063 & 0.499810509 & 0.853843506 \\ 0.499391181 & 0.499367541 & 0.854403390 \\ 0.331204357 & 0.166024982 & 0.980864372 \\ 0.832617132 & 0.166089469 & 0.979506182 \\ 0.333600655 & 0.666872327 & 0.979571554 \\ 0.832446296 & 0.666016595 & 0.979753966\end{array}$

Interpolating coordinate: $0.583(-823.114 \mathrm{eV})$

$\begin{array}{rrr}0.175421589 & 0.009453692 & 0.080547447 \\ 0.329715023 & 0.318277270 & 0.079467261 \\ 0.486540151 & 0.166933141 & 0.082900251 \\ 0.679343978 & 0.014025371 & 0.083320422 \\ 0.833524679 & 0.319974771 & 0.082992296 \\ 0.985173039 & 0.165967129 & 0.082643967 \\ 0.179494098 & 0.513427625 & 0.082816186 \\ 0.332598786 & 0.820277367 & 0.082874171 \\ 0.484959253 & 0.669284159 & 0.082801961 \\ 0.682384602 & 0.513760897 & 0.082920178 \\ 0.832611940 & 0.819568362 & 0.083250073 \\ 0.986277885 & 0.666185397 & 0.082954312 \\ 0.149976455 & 0.152903381 & 0.250087409 \\ -0.000370937 & 0.346858897 & 0.249926097 \\ 0.348745929 & -0.003632652 & 0.250572313 \\ 0.653820892 & 0.153568667 & 0.250169649 \\ 0.505290825 & 0.351321322 & 0.253149850 \\ 0.8466655503 & 0.000470765 & 0.250030564 \\ 0.153821873 & 0.652635216 & 0.249805739 \\ 0.000663967 & 0.847248617 & 0.249678943 \\ 0.346789482 & 0.499885741 & 0.250048361\end{array}$




$\begin{array}{ccc}0.657276430 & 0.657332717 & 0.251856319 \\ 0.500818519 & 0.846234568 & 0.250158300 \\ 0.848211915 & 0.501054577 & 0.250295699 \\ 0.013725225 & 0.180567938 & 0.417082499 \\ 0.320202365 & 0.334036292 & 0.416687384 \\ 0.166726903 & 0.486779293 & 0.416921174 \\ 0.514259054 & 0.180247675 & 0.417431409 \\ 0.820860169 & 0.334112025 & 0.417198043 \\ 0.667893575 & 0.487248881 & 0.417995446 \\ 0.014063664 & 0.680560922 & 0.416797989 \\ 0.319879360 & 0.833028387 & 0.417319866 \\ 0.167352585 & 0.986886772 & 0.416352786 \\ 0.514153363 & 0.680537165 & 0.417113781 \\ 0.821264490 & 0.834894334 & 0.417616461 \\ 0.666835728 & 0.986932614 & 0.417135272 \\ 0.486502617 & 0.319916907 & 0.583409381 \\ 0.180278673 & 0.166711428 & 0.583113607 \\ 0.333417857 & 0.013680267 & 0.583203287 \\ 0.986693451 & 0.319911139 & 0.583405995 \\ 0.680346245 & 0.166980250 & 0.583527478 \\ 0.833264358 & 0.013758489 & 0.583489947 \\ 0.486646252 & 0.819814782 & 0.583505749 \\ 0.180402282 & 0.666819951 & 0.583403273 \\ 0.333304400 & 0.513517114 & 0.583416518 \\ 0.986975262 & 0.820000099 & 0.583390314 \\ 0.680464094 & 0.667035010 & 0.583650889 \\ 0.833596755 & 0.513884162 & 0.583688472 \\ 0.346699329 & 0.346761751 & 0.749580558 \\ 0.152824163 & -0.000296537 & 0.749431511 \\ -0.000328272 & 0.152839937 & 0.749427398 \\ 0.846522103 & 0.346792273 & 0.749670240 \\ 0.652821933 & -0.000158870 & 0.749746299 \\ 0.499824318 & 0.153059144 & 0.749620350 \\ 0.346641981 & 0.846862159 & 0.749659540 \\ 0.152800121 & 0.499791867 & 0.749557860 \\ -0.000084551 & 0.652965427 & 0.749629280 \\ 0.846317535 & 0.846746839 & 0.749703011 \\ 0.652875851 & 0.499935871 & 0.749898650 \\ 0.499854368 & 0.653039886 & 0.749816884 \\ 0.318970341 & 0.486862084 & 0.915404164 \\ 0.165599708 & 0.179794832 & 0.915118153 \\ 0.012347759 & 0.332674197 & 0.915818009 \\ 0.819424317 & 0.486253368 & 0.915994896 \\ 0.666566994 & 0.180014370 & 0.916052104\end{array}$




$\begin{array}{lrl}0.513011798 & 0.332641103 & 0.916297437 \\ 0.319082798 & 0.986244269 & 0.916013440 \\ 0.166341425 & 0.679826658 & 0.915966232 \\ 0.011888012 & 0.831775455 & 0.915313728 \\ 0.819174269 & 0.986181847 & 0.916285111 \\ 0.665847043 & 0.679840159 & 0.916606578 \\ 0.513310310 & 0.833625141 & 0.915978087 \\ 0.165734969 & 0.333204866 & 0.020002461 \\ 0.666108519 & 0.333026203 & 0.020761410 \\ 0.165810746 & 0.832522299 & 0.020217914 \\ 0.665306304 & 0.832954663 & 0.021668867 \\ 0.001495165 & 0.000349364 & 0.145370202 \\ 0.497522579 & 0.000357743 & 0.146592001 \\ 0.000261548 & 0.500499703 & 0.146000199 \\ 0.498821897 & 0.495481993 & 0.145735147 \\ 0.333967944 & 0.171130981 & 0.187584429 \\ 0.832845819 & 0.166150940 & 0.187031608 \\ 0.335544770 & 0.666682378 & 0.186653266 \\ 0.831975575 & 0.666720413 & 0.187557178 \\ 0.166948021 & 0.333647302 & 0.312188670 \\ 0.667524556 & 0.333828646 & 0.312877165 \\ 0.168056911 & 0.833875088 & 0.311432938 \\ 0.667400101 & 0.834290721 & 0.312859387 \\ 0.000782409 & 0.000875490 & 0.353174367 \\ 0.499439772 & -0.000207228 & 0.353530724 \\ 0.000569894 & 0.500683699 & 0.353571901 \\ 0.502143629 & 0.500767040 & 0.352539089 \\ 0.333785159 & 0.167308145 & 0.478580230 \\ 0.833463095 & 0.166924744 & 0.479219985 \\ 0.333893392 & 0.666571580 & 0.479166821 \\ 0.834259231 & 0.667640739 & 0.479333954 \\ 0.166846869 & 0.333443444 & 0.520071895 \\ 0.666976344 & 0.333918553 & 0.520515033 \\ 0.167174221 & 0.833528719 & 0.519974303 \\ 0.666890634 & 0.833525130 & 0.520302425 \\ -0.000035311 & 0.000056014 & 0.645804016 \\ 0.500004300 & 0.000048672 & 0.645896918 \\ 0.000204808 & 0.500100076 & 0.645952447 \\ 0.500065681 & 0.500164605 & 0.646091580 \\ 0.333141876 & 0.166521190 & 0.686902283 \\ 0.833035665 & 0.166588462 & 0.687141266 \\ 0.333224492 & 0.666666016 & 0.687187571 \\ 0.833191807 & 0.666630221 & 0.687263131 \\ 0.166298118 & 0.332913635 & 0.812485872 \\ & & \end{array}$




$\begin{array}{rcc}0.666263344 & 0.333189301 & 0.812895209 \\ 0.166341466 & 0.833253741 & 0.812772505 \\ 0.666103872 & 0.833150035 & 0.813086299 \\ -0.000850593 & -0.000702582 & 0.853762501 \\ 0.499283962 & 0.000057227 & 0.853978211 \\ -0.000332976 & 0.499807549 & 0.853837527 \\ 0.499383755 & 0.499358718 & 0.854393243 \\ 0.331205417 & 0.166022943 & 0.980902230 \\ 0.832609047 & 0.166088601 & 0.979505414 \\ 0.333579888 & 0.666861292 & 0.979566247 \\ 0.832441975 & 0.666023424 & 0.979738049\end{array}$

Interpolating coordinate: 0.667 (-823.145 eV)

$\begin{array}{rrr}0.175120435 & 0.009391869 & 0.080214843 \\ 0.330564943 & 0.319653310 & 0.079241195 \\ 0.486516426 & 0.166888125 & 0.082822901 \\ 0.679292476 & 0.013936033 & 0.083311498 \\ 0.833436538 & 0.319965826 & 0.082946299 \\ 0.985242510 & 0.166074484 & 0.082593459 \\ 0.179487201 & 0.513499736 & 0.082768190 \\ 0.332671248 & 0.820091979 & 0.082810361 \\ 0.485172585 & 0.668674898 & 0.082922897 \\ 0.681955989 & 0.513704671 & 0.082894327 \\ 0.832620927 & 0.819562352 & 0.083176529 \\ 0.986123495 & 0.666100847 & 0.082891483 \\ 0.149561764 & 0.152851754 & 0.250065817 \\ -0.000443383 & 0.346852697 & 0.249884115 \\ 0.348976226 & -0.004231426 & 0.250693429 \\ 0.653837872 & 0.153654903 & 0.250115716 \\ 0.506168288 & 0.352710601 & 0.252914089 \\ 0.846528881 & 0.000387537 & 0.249963255 \\ 0.153780060 & 0.652549281 & 0.249802368 \\ 0.000692252 & 0.847249223 & 0.249610891 \\ 0.346796066 & 0.499858871 & 0.249977060 \\ 0.656989209 & 0.657277977 & 0.251520151 \\ 0.500903614 & 0.846054896 & 0.250093923 \\ 0.848292933 & 0.501177779 & 0.250243450 \\ 0.013697320 & 0.180569961 & 0.417080168 \\ 0.320194717 & 0.333995293 & 0.416639466 \\ 0.166732990 & 0.486764496 & 0.416893761 \\ 0.514257071 & 0.180398335 & 0.417287896 \\ 0.820861816 & 0.334144692 & 0.417174550 \\ 0.667987493 & 0.487362562 & 0.418036033 \\ 0.014086967 & 0.680542572 & 0.416813910 \\ & & \end{array}$




$\begin{array}{ccc}0.319887703 & 0.832974302 & 0.417400525 \\ 0.167360504 & 0.986878317 & 0.416306190 \\ 0.514171378 & 0.680551229 & 0.417118334 \\ 0.821231208 & 0.834819288 & 0.417513836 \\ 0.666858123 & 0.986928465 & 0.417097614 \\ 0.486512847 & 0.319920866 & 0.583376764 \\ 0.180272726 & 0.166702547 & 0.583094384 \\ 0.333447066 & 0.013701268 & 0.583173300 \\ 0.986682255 & 0.319907861 & 0.583399176 \\ 0.680320699 & 0.166983007 & 0.583492053 \\ 0.833269729 & 0.013765786 & 0.583470844 \\ 0.486677563 & 0.819818181 & 0.583514873 \\ 0.180416526 & 0.666816982 & 0.583423469 \\ 0.333289403 & 0.513486752 & 0.583395584 \\ 0.987009765 & 0.820026975 & 0.583382023 \\ 0.680492200 & 0.667072790 & 0.583642509 \\ 0.833602289 & 0.513892532 & 0.583698580 \\ 0.346685122 & 0.346769703 & 0.749545982 \\ 0.152835123 & -0.000261304 & 0.749425897 \\ -0.000338586 & 0.152843652 & 0.749431549 \\ 0.846486593 & 0.346775042 & 0.749662023 \\ 0.652811601 & -0.000167638 & 0.749751734 \\ 0.499790732 & 0.153020950 & 0.749600326 \\ 0.346640104 & 0.846855791 & 0.749672085 \\ 0.152761808 & 0.499795149 & 0.749530482 \\ -0.000104637 & 0.652962963 & 0.749617445 \\ 0.846323423 & 0.846770672 & 0.749688041 \\ 0.652847074 & 0.499924997 & 0.749875585 \\ 0.499864344 & 0.653054812 & 0.749785030 \\ 0.318935858 & 0.486999216 & 0.915266899 \\ 0.165660795 & 0.179899084 & 0.915161247 \\ 0.012313617 & 0.332695563 & 0.915795660 \\ 0.819359467 & 0.486248463 & 0.915991473 \\ 0.666546899 & 0.179990503 & 0.916018727 \\ 0.512971682 & 0.332589626 & 0.916254086 \\ 0.319067205 & 0.986249598 & 0.916019875 \\ 0.166324455 & 0.679808082 & 0.915937236 \\ 0.011814064 & 0.831694478 & 0.915211935 \\ 0.819154159 & 0.986155918 & 0.916286540 \\ 0.665822195 & 0.679823960 & 0.916555909 \\ 0.513281544 & 0.833553647 & 0.916050174 \\ 0.165716917 & 0.333316229 & 0.019911268 \\ 0.665966763 & 0.332930924 & 0.020715327 \\ 0.165783546 & 0.832508678 & 0.020176224\end{array}$




$\begin{array}{ccc}0.665348327 & 0.832953008 & 0.021662424 \\ 0.001605112 & 0.000456162 & 0.145299013 \\ 0.497395752 & 0.000408665 & 0.146638900 \\ 0.000170833 & 0.500421894 & 0.145982939 \\ 0.498586766 & 0.495356157 & 0.145862909 \\ 0.333745493 & 0.171015687 & 0.187712807 \\ 0.832766416 & 0.166076269 & 0.187010903 \\ 0.335420741 & 0.666732504 & 0.186701320 \\ 0.832093302 & 0.666837197 & 0.187487705 \\ 0.166833902 & 0.333562968 & 0.312144416 \\ 0.667526395 & 0.333950726 & 0.312778766 \\ 0.168134096 & 0.833884831 & 0.311430823 \\ 0.667395936 & 0.834287773 & 0.312813635 \\ 0.000801064 & 0.000915958 & 0.353098118 \\ 0.499366446 & -0.000250122 & 0.353505310 \\ 0.000561658 & 0.500688112 & 0.353573504 \\ 0.502185154 & 0.500771769 & 0.352725381 \\ 0.333784021 & 0.167278700 & 0.478531008 \\ 0.833427760 & 0.166924422 & 0.479184006 \\ 0.333884303 & 0.666532110 & 0.479183318 \\ 0.834351211 & 0.667721193 & 0.479344319 \\ 0.166808425 & 0.333408300 & 0.520046441 \\ 0.667003307 & 0.333959446 & 0.520469498 \\ 0.167214150 & 0.833523245 & 0.519957170 \\ 0.666907661 & 0.833556830 & 0.520262764 \\ -0.000041750 & 0.000057984 & 0.645787106 \\ 0.500013628 & 0.000051857 & 0.645882573 \\ 0.000201094 & 0.500099665 & 0.645941652 \\ 0.500079443 & 0.500178894 & 0.646051069 \\ 0.333142784 & 0.166536227 & 0.686864471 \\ 0.833025540 & 0.166585752 & 0.687122418 \\ 0.333221448 & 0.666672857 & 0.687177622 \\ 0.833174766 & 0.666634173 & 0.687249071 \\ 0.166301070 & 0.332952735 & 0.812445345 \\ 0.666188931 & 0.333142733 & 0.812867172 \\ 0.166320742 & 0.833272587 & 0.812742832 \\ 0.666107177 & 0.833134352 & 0.813059492 \\ -0.000794968 & -0.000620632 & 0.853766637 \\ 0.499235020 & 0.000006515 & 0.853990191 \\ -0.000407540 & 0.499792748 & 0.853807631 \\ 0.499346625 & 0.499314601 & 0.854342505 \\ 0.331210720 & 0.166012750 & 0.981091524 \\ 0.832568625 & 0.166084257 & 0.979501570 \\ 0.333476051 & 0.666806118 & 0.979539709\end{array}$


Interpolating coordinate: $0.833(-823.188 \mathrm{eV})$

$\begin{array}{lll}0.174518127 & 0.009268221 & 0.079549634 \\ 0.332264783 & 0.322405390 & 0.078789062 \\ 0.486468974 & 0.166798091 & 0.082668202 \\ 0.679189473 & 0.013757357 & 0.083293650 \\ 0.833260255 & 0.319947937 & 0.082854305 \\ 0.985381451 & 0.166289195 & 0.082492443 \\ 0.179473407 & 0.513643957 & 0.082672196 \\ 0.332816172 & 0.819721202 & 0.082682740 \\ 0.485599248 & 0.667456376 & 0.083164768 \\ 0.681098763 & 0.513592220 & 0.082842624 \\ 0.832638902 & 0.819550331 & 0.083029441 \\ 0.985814716 & 0.665931746 & 0.082765825 \\ 0.148732381 & 0.152748500 & 0.250022633 \\ -0.000588273 & 0.346840298 & 0.249800150 \\ 0.349436821 & -0.005428975 & 0.250935662 \\ 0.653871834 & 0.153827374 & 0.250007851 \\ 0.507923216 & 0.355489159 & 0.252442565 \\ 0.846255638 & 0.000221081 & 0.249828637 \\ 0.153696435 & 0.652377411 & 0.249795625 \\ 0.000748821 & 0.847250436 & 0.249474787 \\ 0.346809232 & 0.499805131 & 0.249834458 \\ 0.656414767 & 0.657168498 & 0.250847817 \\ 0.501073805 & 0.845695553 & 0.249965167 \\ 0.848454969 & 0.501424184 & 0.250138951 \\ 0.013641510 & 0.180574007 & 0.417075506 \\ 0.320179423 & 0.333913296 & 0.416543629 \\ 0.166745163 & 0.486734903 & 0.416838934 \\ 0.514253103 & 0.180699657 & 0.417000870 \\ 0.820865111 & 0.334210026 & 0.417127565 \\ 0.668175331 & 0.487589924 & 0.418117206 \\ 0.014133574 & 0.680505873 & 0.416845752 \\ 0.319904388 & 0.832866132 & 0.417561843 \\ 0.167376342 & 0.986861408 & 0.416212997 \\ 0.514207408 & 0.680579355 & 0.417127439 \\ 0.821164642 & 0.834669197 & 0.417308587 \\ 0.666902912 & 0.986920168 & 0.417022297 \\ 0.486533306 & 0.319928784 & 0.583311531 \\ 0.180260834 & 0.166684787 & 0.583055938 \\ 0.333505482 & 0.013743269 & 0.583113324 \\ 0.986659862 & 0.319901305 & 0.583385538 \\ 0.680269606 & 0.166988520 & 0.583421203 \\ & & \end{array}$




\begin{tabular}{rrr}
0.833280471 & 0.013780380 & 0.583432639 \\
0.486740184 & 0.819824978 & 0.583533121 \\
0.180445014 & 0.666811044 & 0.583463863 \\
0.333259408 & 0.513426029 & 0.583353717 \\
0.987078771 & 0.820080726 & 0.583365441 \\
0.680548412 & 0.667148351 & 0.583625751 \\
0.833613358 & 0.513909272 & 0.583718794 \\
0.346656710 & 0.346785607 & 0.749476829 \\
0.152857041 & -0.000190837 & 0.749414671 \\
-0.000359214 & 0.152851082 & 0.749439851 \\
0.846415572 & 0.346740581 & 0.749645590 \\
0.652790937 & -0.000185176 & 0.749762603 \\
0.499723560 & 0.152944562 & 0.749560278 \\
0.346636350 & 0.846843056 & 0.749697177 \\
0.152685182 & 0.499801713 & 0.749475728 \\
-0.000144810 & 0.652958035 & 0.749593775 \\
0.846335200 & 0.846818337 & 0.749658100 \\
0.652789518 & 0.499903249 & 0.749829456 \\
0.499884296 & 0.653084662 & 0.749721322 \\
0.318866894 & 0.487273481 & 0.914992367 \\
0.165782967 & 0.180107588 & 0.915247436 \\
0.012245333 & 0.332738296 & 0.915750963 \\
0.819229766 & 0.486238652 & 0.915984628 \\
0.666506709 & 0.179942768 & 0.915951973 \\
0.512891450 & 0.332486672 & 0.916167385 \\
0.319036020 & 0.986260255 & 0.916032745 \\
0.166290516 & 0.679770929 & 0.915879244 \\
0.011666169 & 0.831532525 & 0.915008349 \\
0.819113939 & 0.986104061 & 0.916289398 \\
0.665772499 & 0.679791562 & 0.916454569 \\
0.513224013 & 0.833410658 & 0.916194348 \\
0.165680811 & 0.333538956 & 0.019728881 \\
0.665683250 & 0.332740365 & 0.020623161 \\
0.165729145 & 0.832481435 & 0.020092845 \\
0.665432373 & 0.832949698 & 0.021649539 \\
0.001825007 & 0.000669758 & 0.145156635 \\
0.497142098 & 0.000510510 & 0.146732697 \\
-0.000010595 & 0.500266277 & 0.145948419 \\
0.498116503 & 0.495104487 & 0.146118431 \\
0.333300592 & 0.170785099 & 0.187969564 \\
0.832607610 & 0.165926927 & 0.186969492 \\
0.335172682 & 0.666832755 & 0.186797426 \\
0.832328756 & 0.667070764 & 0.187348758 \\
0.166605666 & 0.333394300 & 0.312055908 \\
\hline & &
\end{tabular}




\begin{tabular}{|c|c|c|}
\hline 0.667530074 & 0.334194886 & 0.312581968 \\
\hline 0.168288465 & 0.833904315 & 0.311426593 \\
\hline 0.667387605 & 0.834281877 & 0.312722130 \\
\hline 0.000838375 & 0.000996894 & 0.352945619 \\
\hline 0.499219794 & -0.000335910 & 0.353454483 \\
\hline 0.000545188 & 0.500696938 & 0.353576711 \\
\hline 0.502268204 & 0.500781228 & 0.353097965 \\
\hline 0.333781744 & 0.167219809 & 0.478432563 \\
\hline 0.833357089 & 0.166923779 & 0.479112047 \\
\hline 0.333866123 & 0.666453170 & 0.479216314 \\
\hline 0.834535171 & 0.667882101 & 0.479365050 \\
\hline 0.166731538 & 0.333338013 & 0.519995532 \\
\hline 0.667057234 & 0.334041232 & 0.520378427 \\
\hline 0.167294008 & 0.833512296 & 0.519922903 \\
\hline 0.666941714 & 0.833620231 & 0.520183444 \\
\hline-0.000054629 & 0.000061923 & 0.645753286 \\
\hline 0.500032284 & 0.000058228 & 0.645853884 \\
\hline 0.000193668 & 0.500098844 & 0.645920061 \\
\hline 0.500106967 & 0.500207470 & 0.645970048 \\
\hline 0.333144600 & 0.166566301 & 0.686788846 \\
\hline 0.833005290 & 0.166580331 & 0.687084721 \\
\hline 0.333215359 & 0.666686539 & 0.687157724 \\
\hline 0.833140684 & 0.666642077 & 0.687220949 \\
\hline 0.166306973 & 0.333030935 & 0.812364292 \\
\hline 0.666040107 & 0.333049598 & 0.812811098 \\
\hline 0.166279295 & 0.833310279 & 0.812683486 \\
\hline 0.666113787 & 0.833102987 & 0.813005878 \\
\hline-0.000683717 & -0.000456731 & 0.853774910 \\
\hline 0.499137136 & -0.000094908 & 0.854014151 \\
\hline-0.000556668 & 0.499763146 & 0.853747840 \\
\hline 0.499272365 & 0.499226368 & 0.854241030 \\
\hline 0.331221324 & 0.165992365 & 0.981470111 \\
\hline 0.832487780 & 0.166075570 & 0.979493884 \\
\hline 0.333268377 & 0.666695769 & 0.979486634 \\
\hline 0.832377174 & 0.666125864 & 0.979499300 \\
\hline
\end{tabular}

Final coordinate: 1 (-823.203 eV)

$\begin{array}{lll}0.1799716661305856 & 0.0132731111377156 & 0.0828007687726189 \\ 0.3348785804450521 & 0.3258438193510401 & 0.0791522773875764 \\ 0.4908750404109400 & 0.1657108688485535 & 0.0785785417464477 \\ 0.6798654746762419 & 0.0137569355045823 & 0.0835706634827674 \\ 0.8340925781555830 & 0.3203341311861355 & 0.0827972069254225 \\ 0.9860631298278936 & 0.1668069193950552 & 0.0827181721092033 \\ 0.1799037743070672 & 0.5142545913814115 & 0.0828310700038620\end{array}$




\begin{tabular}{|c|c|c|}
\hline & & \\
\hline & & \\
\hline 932 & 1 & \\
\hline & & \\
\hline 295 & 471 & 644 \\
\hline 85081 & 433 & 0.25 \\
\hline & & \\
\hline & & \\
\hline 0.65 & 62 & \\
\hline 0.5 & & \\
\hline & & \\
\hline 632 & & \\
\hline 60 & & \\
\hline 2 & & \\
\hline & & \\
\hline 0.50 & 03 & \\
\hline 0.84 & & \\
\hline & & \\
\hline 0.31 & 39 & \\
\hline 0.16 & 0.4 & \\
\hline & & \\
\hline 0.8 & & \\
\hline 0.6 & 0.4 & \\
\hline 609 & 425 & \\
\hline & & \\
\hline & & \\
\hline 19 & 73 & \\
\hline 0.81 & & \\
\hline 0.66 & 0.98 & \\
\hline & & \\
\hline 0.180 & 0.166 & 0.5 \\
\hline 0.3 & 83 & 0.5 \\
\hline & & \\
\hline 0.68 & 38 & 058 \\
\hline 0.833 & 92 & \\
\hline & & \\
\hline & & \\
\hline 0.333 & $0.513-2-2 \cdot-3$ & \\
\hline & 0.8198508661377766 & \\
\hline & 357849397 & 0.58 \\
\hline 0.8331945091818393 & 0.5134967680714569 & 0.5835935164103 \\
\hline 7229 & 0.3469127877186254 & 0.7496 \\
\hline 73285 & 0.0000211004791308 & 0.7497545024496500 \\
\hline $.000027 / 10000004$ & 0.1350030005350000 & 0.145514503004000 \\
\hline
\end{tabular}




\begin{tabular}{|c|c|c|}
\hline & & \\
\hline 3031617 & 9913 & 0.749 \\
\hline 1998638332598784 & 0.1529870 & \\
\hline 631460 & 74451 & 19 \\
\hline 1635 & 531822 & 27 \\
\hline 5602 & 8654 & 9581 \\
\hline 8468241783314547 & 0.8469432480334440 & 7523 \\
\hline & 0. & \\
\hline 458 & 0.653 & 615 \\
\hline 0905 & 0.4880 & 515 \\
\hline 73 & 0. & 48 \\
\hline & 0.333 & \\
\hline 000 & 0844 & 0274 \\
\hline 512 & 3042 & 302 \\
\hline & 0.3 & \\
\hline 9 & 8 & 90 \\
\hline 2168549 & 0.6804 & 1186 \\
\hline 9611 & 0.83 & 617 \\
\hline 34 & $0 . \subseteq$ & \\
\hline 939 & 0.680 & 250 \\
\hline 7533 & 0.8338 & 723 \\
\hline 452 & 0.3 & 313 \\
\hline & & \\
\hline & 03 & \\
\hline 6670769648598021 & 0.834139775 & 3669 \\
\hline 3 & 0. & \\
\hline & & \\
\hline 8726 & 581824664 & 5711 \\
\hline 49861165 & 0.4977974387451381 & 4392 \\
\hline 339 & 0.1672 & 1564 \\
\hline & 0.165070840523 & \\
\hline 3344911923033786 & 0.6673669745767467 & 0.1874581 \\
\hline 3331772135238082 & 0.6675768 & 24531 \\
\hline & 0.33 & \\
\hline 6667661395313577 & 0.3338442268499978 & 0.3122678795979730 \\
\hline 0.1667441160694176 & 0.8325554010574420 & 0.3126436754754621 \\
\hline 70632 & 0.832 & 324 \\
\hline 0003648692492117 & 0.0010722405167379 & 0.3535591965939246 \\
\hline 0.49840580191455 & .0020361156584462 & 0.3537295514093773 \\
\hline 0001299343153898 & 0.4996882672171326 & 0.3538390765145197 \\
\hline 001520459488793 & 0.4993849208941228 & 0.3530645624648558 \\
\hline 0.3333140901992270 & 0.1664343316383954 & 0.4785615312413503 \\
\hline 17 & 0.166 & 0.479 \\
\hline 0.3333643537323379 & 0.6655572424264247 & 0.4796023361267055 \\
\hline
\end{tabular}




$\begin{array}{ccc}0.8336207638864643 & 0.6670153725287946 & 0.4792990859153017 \\ 0.1661789577624963 & 0.3328634161381144 & 0.5200863492987715 \\ 0.6665815021370077 & 0.3335284239218443 & 0.5202484239496692 \\ 0.1669798107479412 & 0.8331017205558154 & 0.5205169930022896 \\ 0.6667087650429533 & 0.8332589847563081 & 0.5203175485465340 \\ -0.0000149432438960 & -0.0000058823002184 & 0.6460646791995808 \\ 0.5000316587622675 & -0.0001165682676823 & 0.6461601078142327 \\ -0.0001529786998937 & 0.4998589989697137 & 0.6460522246203043 \\ 0.5000568623278028 & 0.5000081381657319 & 0.6460052862596591 \\ 0.3332531034623312 & 0.1666090539046626 & 0.6869418359648607 \\ 0.8332331490777057 & 0.1665510975200944 & 0.6873378546133346 \\ 0.3333450271729540 & 0.6666948625072345 & 0.6874000637056965 \\ 0.8334269544105219 & 0.6668738230556800 & 0.6872846963461499 \\ 0.1668170990478095 & 0.3333529982493560 & 0.8127991702731322 \\ 0.6665161710476593 & 0.33334438888499054 & 0.8125511740035878 \\ 0.1667738085099159 & 0.8336866200011253 & 0.8130729279028570 \\ 0.6666821201456012 & 0.8335111001676763 & 0.8131957566291851 \\ 0.0004805008802520 & 0.0004818786496796 & 0.8542760377610062 \\ 0.4995908856540410 & 0.0003562352917708 & 0.8544070290477866 \\ 0.0001108539459442 & 0.5002460416564849 & 0.8538201144679243 \\ 0.4998663470880770 & 0.5001237887812817 & 0.8540922479964299 \\ 0.3344157624586796 & 0.1683984975322698 & 0.9821415199436866 \\ 0.8331787777290417 & 0.1664480878433171 & 0.9796003864952514 \\ 0.3334537808214362 & 0.6672808151648340 & 0.9795782650318268 \\ 0.8332786451130790 & 0.6671672662016915 & 0.9797350256407779\end{array}$

(1.10) $2 \times 2 \times 1$ supercell with the typical snapshots chosen from ab initio molecular dynamics simulations Snapshot: 5 ps (-817.610 eV)
$\begin{array}{lll}0.16501878 & 0.99554580 & 0.07420319\end{array}$
$\begin{array}{llll}0.32462780 & 0.30615644 & 0.10108273\end{array}$
$\begin{array}{llll}0.49114158 & 0.15662219 & 0.06885337\end{array}$
$\begin{array}{lll}0.68336031 & 0.00605488 & 0.09128336\end{array}$
$\begin{array}{llll}0.82746435 & 0.32017699 & 0.08771868\end{array}$
$\begin{array}{llll}0.98993180 & 0.17099246 & 0.09120828\end{array}$
$\begin{array}{llll}0.18920870 & 0.52086888 & 0.08261965\end{array}$
$\begin{array}{llll}0.33650549 & 0.81396526 & 0.08567995\end{array}$
$\begin{array}{llll}0.48681802 & 0.65208587 & 0.06659887\end{array}$
$\begin{array}{llll}0.68607701 & 0.52017293 & 0.09121969\end{array}$
$\begin{array}{lll}0.83871914 & 0.81777555 & 0.08508062\end{array}$
$\begin{array}{llll}0.98761929 & 0.66681342 & 0.08805249\end{array}$
$\begin{array}{llll}0.13511942 & 0.14464535 & 0.25292547\end{array}$
$\begin{array}{llll}0.99614691 & 0.34310831 & 0.25560422\end{array}$
$\begin{array}{llll}0.35146986 & 0.00228796 & 0.24191665\end{array}$
$\begin{array}{llll}0.65389079 & 0.15294474 & 0.24980150\end{array}$ 


\begin{tabular}{|c|c|c|}
\hline 363 & 0.35 & 0 \\
\hline 0.84257030 & 0.00358926 & 0.24842381 \\
\hline 16637897 & 0.65866560 & 25977370 \\
\hline 01318329 & 0.84837786 & 5254545 \\
\hline 34779552 & 0.50078504 & 0.25012546 \\
\hline 0.65627683 & 0.66230359 & 0.24840346 \\
\hline 0.48881903 & 0.83864652 & 0.25525461 \\
\hline 37057 & 10196 & 753 \\
\hline 0.02067333 & 0.19287976 & 0.41803066 \\
\hline 0.32780409 & 0.33089693 & 0.41480521 \\
\hline 17253160 & 0.48435937 & 0.40999959 \\
\hline 0.50781943 & 0.17379046 & 0.41814549 \\
\hline 0.81311085 & 0.32841934 & 0.41631649 \\
\hline 0.67043261 & 0.49042137 & 0.41572433 \\
\hline 0.01 & 0.67380912 & 0.42 \\
\hline 0.32535617 & 0.84270652 & 2234 \\
\hline 0.15615834 & 0.98096047 & 0.41778309 \\
\hline 51857369 & 0.68787466 & 0.41136385 \\
\hline 39 & 0.83 & \\
\hline 0.65625858 & 0.98351516 & 0.42024290 \\
\hline 0.48031018 & 0.31553815 & 0.57633921 \\
\hline 17641699 & 0.15968289 & $0.57 c$ \\
\hline 0.32919049 & 0.00401830 & 0.58835308 \\
\hline 0.98341333 & 0.32231490 & 0.57392248 \\
\hline 0.66414751 & 0.15932431 & 0.58013717 \\
\hline 0.83647857 & 0.01573768 & 0.58431132 \\
\hline 0.47080579 & 0.81394591 & 0.58522114 \\
\hline 0.16801667 & 0.65699119 & 0.58490618 \\
\hline 0.33315086 & 0.51081925 & 0.57618381 \\
\hline 0.99675198 & 0.81692681 & 0.57979735 \\
\hline 0.67168673 & 0.65817187 & 0.58169323 \\
\hline 0.81969159 & 0.50717269 & 0.57679109 \\
\hline 0.34013280 & 0.34289251 & 0.74833108 \\
\hline 0.13959498 & 0.99473316 & 0.75437845 \\
\hline 0.01242769 & 0.17001382 & 0.74818837 \\
\hline 0.84594253 & 0.34406814 & 0.75521919 \\
\hline 0.65700591 & 0.00061013 & 0.75470954 \\
\hline 0.49643035 & 0.15184022 & 0.75579606 \\
\hline 0.34978211 & 0.84307599 & 0.74863126 \\
\hline 0.14544480 & 0.48307266 & 0.73486036 \\
\hline 0.00794901 & 0.65761124 & 0.74238226 \\
\hline 0.84443770 & 0.85861096 & 0.73843057 \\
\hline 0.65334056 & 0.49570362 & 0.74335301 \\
\hline 0.514589 & 0.66376 & .7433 \\
\hline
\end{tabular}




\begin{tabular}{lll}
0.32201747 & 0.49412532 & 0.90877893 \\
0.16278272 & 0.18331151 & 0.92523807 \\
0.00753758 & 0.33766256 & 0.92459811 \\
0.81415968 & 0.49693164 & 0.91142015 \\
0.68064834 & 0.18510894 & 0.91749597 \\
0.51769282 & 0.34863116 & 0.92225874 \\
0.31528687 & 0.98237743 & 0.91844313 \\
0.16961546 & 0.68410107 & 0.91353192 \\
0.01029260 & 0.83258248 & 0.91206225 \\
0.83534852 & 0.98625071 & 0.92323953 \\
0.66611738 & 0.68021399 & 0.91057499 \\
0.51720152 & 0.83866586 & 0.91019223 \\
0.18252393 & 0.33180970 & 0.02327778 \\
0.66144578 & 0.33638366 & 0.02271276 \\
0.15575692 & 0.81647284 & 0.01868066 \\
0.66928947 & 0.83101242 & 0.01843742 \\
0.00442262 & 0.99502515 & 0.15048963 \\
0.49847352 & 0.99887881 & 0.14856525 \\
0.01188582 & 0.49640518 & 0.14392906 \\
0.48956642 & 0.49690370 & 0.15188714 \\
0.33841612 & 0.17379814 & 0.20528604 \\
0.83681758 & 0.17079299 & 0.18798102 \\
0.34173504 & 0.66907097 & 0.18880965 \\
0.83264216 & 0.66991555 & 0.19187222 \\
0.16532529 & 0.33305378 & 0.30968823 \\
0.66148449 & 0.33295684 & 0.30715010 \\
0.17459819 & 0.84004270 & 0.31025105 \\
0.66816328 & 0.83539418 & 0.31639512 \\
0.99694059 & 0.00561275 & 0.35474669 \\
0.49422057 & 0.99883578 & 0.35206703 \\
0.99769472 & 0.50579503 & 0.34764627 \\
0.50437385 & 0.50603609 & 0.35348278 \\
0.31834750 & 0.16598121 & 0.47148830 \\
0.82565250 & 0.15681764 & 0.48139747 \\
0.32981094 & 0.66568951 & 0.47921595 \\
0.82729118 & 0.66393396 & 0.47943975 \\
0.15695087 & 0.32943849 & 0.51550967 \\
0.65593796 & 0.32550273 & 0.51731429 \\
0.17360591 & 0.83481892 & 0.51981127 \\
0.55844528 & 0.82140710 & 0.52281168 \\
\hline & 0.00000422 & 0.64271634 \\
\hline
\end{tabular}




$\begin{array}{lll}0.33738324 & 0.16703610 & 0.68449109 \\ 0.84336576 & 0.16974222 & 0.69178532 \\ 0.33081361 & 0.67011940 & 0.68213108 \\ 0.84875605 & 0.67556158 & 0.68303141 \\ 0.16377462 & 0.34244979 & 0.82026045 \\ 0.66441242 & 0.33237693 & 0.80681435 \\ 0.17794540 & 0.84231404 & 0.80923606 \\ 0.66746471 & 0.83399134 & 0.81126229 \\ 0.99943126 & 0.99629358 & 0.85635917 \\ 0.50412150 & 0.00363355 & 0.86775793 \\ 0.99640460 & 0.51071061 & 0.85447142 \\ 0.49620287 & 0.49864968 & 0.84536601 \\ 0.33160393 & 0.15549771 & 0.99262455 \\ 0.84015489 & 0.16485807 & 0.98250196 \\ 0.34655085 & 0.68565581 & 0.97652551 \\ 0.82194637 & 0.66621509 & 0.97726858\end{array}$

Snapshot: 9.5 ps (-818.601 eV)

$\begin{array}{llll}0.18684197 & 0.02562763 & 0.08306683\end{array}$

$\begin{array}{lll}0.33632205 & 0.33634652 & 0.08108277\end{array}$

$\begin{array}{llll}0.47901388 & 0.17450809 & 0.08529500\end{array}$

$\begin{array}{lll}0.68471386 & 0.00396830 & 0.09077348\end{array}$

$\begin{array}{llll}0.81500768 & 0.31529652 & 0.08174240\end{array}$

$\begin{array}{llll}0.98545148 & 0.16295147 & 0.07782186\end{array}$

$\begin{array}{llll}0.17711570 & 0.50941351 & 0.08346864\end{array}$

$\begin{array}{llll}0.32632664 & 0.81115730 & 0.07834537\end{array}$

$\begin{array}{llll}0.48579555 & 0.66890089 & 0.08437604\end{array}$

$\begin{array}{llll}0.68076785 & 0.51921502 & 0.08955995\end{array}$

$\begin{array}{llll}0.84811920 & 0.82134206 & 0.08054064\end{array}$

$\begin{array}{llll}0.98675255 & 0.66967485 & 0.08505246\end{array}$

$\begin{array}{lll}0.15097896 & 0.16009482 & 0.24489038\end{array}$

$\begin{array}{llll}0.98625590 & 0.33699185 & 0.24714731\end{array}$

$\begin{array}{llll}0.35059752 & 0.01473477 & 0.25666863\end{array}$

$\begin{array}{llll}0.64763045 & 0.14199148 & 0.24732620\end{array}$

$\begin{array}{llll}0.49805774 & 0.35214578 & 0.25068486\end{array}$

$\begin{array}{llll}0.84135098 & 0.98698518 & 0.24782427\end{array}$

$\begin{array}{llll}0.15685943 & 0.65410155 & 0.24956705\end{array}$

$\begin{array}{llll}0.99507438 & 0.84077808 & 0.24919196\end{array}$

$\begin{array}{llll}0.33705660 & 0.49692913 & 0.25767205\end{array}$

$\begin{array}{lll}0.65245297 & 0.64776153 & 0.25021209\end{array}$

$\begin{array}{lll}0.48736011 & 0.84119969 & 0.25936488\end{array}$

$\begin{array}{llll}0.85317194 & 0.49153503 & 0.24598442\end{array}$

$\begin{array}{llll}0.01603422 & 0.17661415 & 0.41471219\end{array}$

$\begin{array}{llll}0.31558171 & 0.32527626 & 0.41136958\end{array}$ 


\begin{tabular}{lll}
0.15982125 & 0.48959829 & 0.41211859 \\
0.51590520 & 0.18581709 & 0.41624983 \\
0.81326678 & 0.32965989 & 0.42046738 \\
0.66234399 & 0.49302218 & 0.41686587 \\
0.01122771 & 0.67797793 & 0.42090166 \\
0.32540128 & 0.84120111 & 0.42265120 \\
0.16387298 & 0.98692836 & 0.41749935 \\
0.51777758 & 0.68565480 & 0.41814542 \\
0.81092292 & 0.83051531 & 0.41782803 \\
0.67192861 & 0.99848520 & 0.41521465 \\
0.48578149 & 0.31856722 & 0.58857329 \\
0.19037594 & 0.16682972 & 0.58303920 \\
0.33230723 & 0.00296793 & 0.59153853 \\
0.98550310 & 0.31608786 & 0.57786414 \\
0.67316393 & 0.16061992 & 0.58760384 \\
0.82571380 & 0.01245494 & 0.58020534 \\
0.48229056 & 0.81540348 & 0.59252409 \\
0.18366133 & 0.66859914 & 0.58478841 \\
0.34031940 & 0.50672940 & 0.57530675 \\
0.98598890 & 0.82510806 & 0.58373395 \\
0.67916753 & 0.67571252 & 0.58621219 \\
0.82651043 & 0.51226197 & 0.58206579 \\
0.35484074 & 0.34845336 & 0.76198136 \\
0.15855140 & 0.99893368 & 0.75395187 \\
0.00399363 & 0.15870459 & 0.74395842 \\
0.84657263 & 0.35016194 & 0.74532602 \\
0.65371605 & 0.99687292 & 0.74974283 \\
0.49340926 & 0.16045827 & 0.75843754 \\
0.35310232 & 0.84867764 & 0.74646597 \\
0.15790467 & 0.50164620 & 0.74003870 \\
0.00726101 & 0.65638643 & 0.74509389 \\
0.85903712 & 0.85677803 & 0.75310248 \\
0.65595894 & 0.50730328 & 0.75207127 \\
0.50114169 & 0.65069622 & 0.75630624 \\
0.30785653 & 0.49310283 & 0.92393919 \\
0.16777020 & 0.17987109 & 0.91485137 \\
0.00518599 & 0.32578559 & 0.90904823 \\
0.82596333 & 0.47923207 & 0.91118153 \\
0.5657575 & 0.17508563 & 0.91585337 \\
\hline & 0.32701107 & 0.91834546 \\
\hline
\end{tabular}




\begin{tabular}{lll}
0.67919420 & 0.68210534 & 0.91733317 \\
0.51833757 & 0.83970672 & 0.91442545 \\
0.17141446 & 0.33522507 & 0.02334377 \\
0.65343532 & 0.32499146 & 0.01864895 \\
0.17117452 & 0.84415052 & 0.02172999 \\
0.67613126 & 0.83557776 & 0.02281877 \\
0.99507752 & 0.99957425 & 0.13988576 \\
0.50749086 & 0.00160568 & 0.13786772 \\
0.99445060 & 0.48559108 & 0.14660942 \\
0.51581276 & 0.51019264 & 0.14190109 \\
0.33340658 & 0.17573470 & 0.18884886 \\
0.82233286 & 0.15080905 & 0.18620359 \\
0.33654862 & 0.66080189 & 0.19217785 \\
0.83527950 & 0.65935891 & 0.19482868 \\
0.15241137 & 0.32818720 & 0.31089794 \\
0.66174362 & 0.33041976 & 0.30704239 \\
0.17440685 & 0.83935255 & 0.31020759 \\
0.66417169 & 0.83260977 & 0.30762509 \\
0.99436617 & 0.99097809 & 0.35050618 \\
0.49283339 & 0.99509484 & 0.35659484 \\
0.98793550 & 0.49628082 & 0.35840561 \\
0.49163613 & 0.49030716 & 0.35802272 \\
0.33535256 & 0.16776547 & 0.48020870 \\
0.83003065 & 0.16649595 & 0.47352013 \\
0.32864800 & 0.66137095 & 0.48054840 \\
0.82914399 & 0.67014788 & 0.47563851 \\
0.16843530 & 0.33799169 & 0.51342820 \\
0.65831609 & 0.32938538 & 0.51566289 \\
0.16661557 & 0.82981835 & 0.52263080 \\
0.65885760 & 0.83051900 & 0.51776606 \\
0.00250719 & 0.01297411 & 0.64553669 \\
0.50300962 & 0.00065279 & 0.65113033 \\
0.98855531 & 0.49535928 & 0.64389963 \\
0.49833598 & 0.49841438 & 0.64756928 \\
0.33945330 & 0.16599121 & 0.69057148 \\
0.83435090 & 0.17039410 & 0.68278918 \\
0.34127373 & 0.67312748 & 0.69603652 \\
0.83413426 & 0.66908857 & 0.68838352 \\
0.17024692 & 0.32915709 & 0.81706144 \\
0.66965772 & 0.33818680 & 0.81077573 \\
\hline & 0.83377129 & 0.81327812 \\
\hline
\end{tabular}




$\begin{array}{lll}0.01841324 & 0.50465331 & 0.84793181 \\ 0.50072026 & 0.50720895 & 0.86260959 \\ 0.33352789 & 0.16490292 & 0.97641567 \\ 0.83356285 & 0.17081773 & 0.97770142 \\ 0.34489575 & 0.68308506 & 0.98035113 \\ 0.84335288 & 0.65077238 & 0.98417499\end{array}$

Snapshot: 25 ps (-818.481 eV)

$\begin{array}{lll}0.17090008 & 0.01414639 & 0.08594380 \\ 0.34756706 & 0.31879970 & 0.07679416 \\ 0.47110556 & 0.15761502 & 0.08718944 \\ 0.67207011 & 0.01292159 & 0.08378425 \\ 0.83381822 & 0.31658886 & 0.08131087 \\ 0.98829278 & 0.16218630 & 0.08285903 \\ 0.19171169 & 0.52536216 & 0.08457132 \\ 0.32078522 & 0.81599311 & 0.09164627 \\ 0.48583342 & 0.67777106 & 0.07709149 \\ 0.68684157 & 0.51067051 & 0.08911150 \\ 0.82978026 & 0.81910494 & 0.07801647 \\ 0.99489963 & 0.67582350 & 0.08917857 \\ 0.15130608 & 0.15088577 & 0.24984103 \\ 0.01897232 & 0.37255143 & 0.24960333 \\ 0.33557244 & 0.00102701 & 0.25802118 \\ 0.62967751 & 0.15501635 & 0.25088068 \\ 0.48876312 & 0.34449108 & 0.24680407 \\ 0.84007702 & 0.00127916 & 0.25102342 \\ 0.15253846 & 0.66282084 & 0.24993555 \\ 0.98921455 & 0.86071811 & 0.24827531 \\ 0.34797791 & 0.50181549 & 0.25028268 \\ 0.65095042 & 0.64641213 & 0.25179104 \\ 0.50273863 & 0.84505090 & 0.25126583 \\ 0.84216731 & 0.49730236 & 0.25145165 \\ 0.00897100 & 0.19006818 & 0.40756850 \\ 0.32524262 & 0.33861382 & 0.42358694 \\ 0.17784337 & 0.49771742 & 0.41472984 \\ 0.51849414 & 0.19263977 & 0.41252926 \\ 0.82060805 & 0.33398570 & 0.41057244 \\ 0.66569743 & 0.48449013 & 0.42695504 \\ 0.00850790 & 0.67171756 & 0.41734985 \\ 0.32385480 & 0.83798962 & 0.41875905 \\ 0.16230470 & 0.98640016 & 0.42346107 \\ 0.53428901 & 0.69226628 & 0.41237088 \\ 0.81973473 & 0.84204567 & 0.42290397 \\ 0.65889363 & 0.97706891 & 0.42015651\end{array}$




\begin{tabular}{lll}
0.49067937 & 0.32713287 & 0.58557994 \\
0.18839679 & 0.16602996 & 0.58326502 \\
0.34084512 & 0.02084248 & 0.58235134 \\
0.97987539 & 0.31705209 & 0.57678795 \\
0.68310757 & 0.15944490 & 0.57536988 \\
0.84321564 & 0.02339268 & 0.58597632 \\
0.50511335 & 0.82723844 & 0.59078709 \\
0.18944822 & 0.66494839 & 0.58714768 \\
0.33436333 & 0.51344122 & 0.58661072 \\
0.98840485 & 0.81594366 & 0.57920872 \\
0.68633737 & 0.65925064 & 0.58429334 \\
0.82416830 & 0.49706502 & 0.58839276 \\
0.34558060 & 0.34618558 & 0.74891131 \\
0.16606132 & 0.00089759 & 0.75016331 \\
0.00922612 & 0.15347574 & 0.74531672 \\
0.84616123 & 0.34273809 & 0.75097258 \\
0.65472573 & 0.99708712 & 0.74557391 \\
0.50230661 & 0.15389925 & 0.74706721 \\
0.35210534 & 0.85455523 & 0.74373580 \\
0.15673411 & 0.49678199 & 0.75280472 \\
0.01126855 & 0.65037860 & 0.74095305 \\
0.84248970 & 0.84568457 & 0.75308921 \\
0.65387339 & 0.50451524 & 0.75699809 \\
0.48915246 & 0.65416233 & 0.75234914 \\
0.30631066 & 0.47435361 & 0.91884995 \\
0.16996320 & 0.18209253 & 0.91980103 \\
0.01055797 & 0.32549311 & 0.92075338 \\
0.81333173 & 0.47760273 & 0.91520304 \\
0.66374842 & 0.16871455 & 0.91832042 \\
0.50655477 & 0.32495411 & 0.91208095 \\
0.32054893 & 0.97859651 & 0.91563432 \\
0.15973656 & 0.67664453 & 0.91999478 \\
0.00871047 & 0.84146587 & 0.91783897 \\
0.81063117 & 0.97981539 & 0.91387458 \\
0.66268539 & 0.66490445 & 0.91261520 \\
0.51877110 & 0.83144441 & 0.91320045 \\
0.16426279 & 0.32429199 & 0.02365889 \\
0.66327285 & 0.32796480 & 0.02360623 \\
0.16960201 & 0.83948724 & 0.01916537 \\
\hline & 0.83097413 & 0.01869287 \\
\hline
\end{tabular}




$\begin{array}{lll}0.32230358 & 0.15515989 & 0.18422701 \\ 0.81999516 & 0.16320726 & 0.19418735 \\ 0.33040874 & 0.67330050 & 0.19400365 \\ 0.82817001 & 0.65697076 & 0.18975586 \\ 0.17395069 & 0.33850396 & 0.30954620 \\ 0.65466528 & 0.33928660 & 0.30548526 \\ 0.15980863 & 0.83965974 & 0.31708031 \\ 0.66486826 & 0.83571769 & 0.31117715 \\ 0.99417660 & 0.99200913 & 0.35250083 \\ 0.50275076 & 0.01356288 & 0.35465056 \\ 0.02048240 & 0.50970268 & 0.34785937 \\ 0.49868345 & 0.49800535 & 0.35288564 \\ 0.34293233 & 0.17814211 & 0.48306942 \\ 0.84012205 & 0.17312597 & 0.46917107 \\ 0.34773702 & 0.67345701 & 0.48261361 \\ 0.83447023 & 0.67039896 & 0.47729542 \\ 0.16407837 & 0.33720033 & 0.52131901 \\ 0.67357153 & 0.33588497 & 0.51419487 \\ 0.18372822 & 0.83699803 & 0.52233820 \\ 0.68080751 & 0.84471527 & 0.52983436 \\ 0.00568905 & 0.00230666 & 0.63928048 \\ 0.50882826 & 0.00530631 & 0.64313405 \\ 0.00052182 & 0.48893494 & 0.64146156 \\ 0.50489314 & 0.50912577 & 0.64887672 \\ 0.33634404 & 0.16765168 & 0.68696867 \\ 0.82298420 & 0.15721583 & 0.68497290 \\ 0.32624122 & 0.66681262 & 0.68713521 \\ 0.83974124 & 0.66218595 & 0.69673909 \\ 0.16570288 & 0.33127685 & 0.81208265 \\ 0.66677615 & 0.33130204 & 0.81069724 \\ 0.17087619 & 0.83655550 & 0.80994224 \\ 0.65901695 & 0.82776408 & 0.81254566 \\ 0.00005445 & 0.99832188 & 0.85390375 \\ 0.49587305 & 0.99858459 & 0.84968597 \\ 0.99524664 & 0.49220923 & 0.85899398 \\ 0.49291773 & 0.49697103 & 0.85769446 \\ 0.33120516 & 0.15436851 & 0.97703129 \\ 0.83107475 & 0.16381796 & 0.98407443 \\ 0.33043954 & 0.65784014 & 0.98067787 \\ 0.83447224 & 0.66369830 & 0.98008838 \\ & & \end{array}$

Snapshot: 37.5 ps (-818.210 eV)

$\begin{array}{lll}0.19193802 & 0.01924579 & 0.08049646\end{array}$

$\begin{array}{lll}0.32645358 & 0.31254775 & 0.08250722\end{array}$ 


\begin{tabular}{lll}
0.48203952 & 0.16246722 & 0.08331357 \\
0.67998819 & 0.01941001 & 0.07742860 \\
0.83135910 & 0.31972121 & 0.08875437 \\
0.97597081 & 0.16984450 & 0.08189932 \\
0.16768435 & 0.51305323 & 0.08408478 \\
0.33389512 & 0.81566735 & 0.08489118 \\
0.47961871 & 0.66639936 & 0.08701565 \\
0.67406095 & 0.50972461 & 0.07542997 \\
0.83577435 & 0.82359135 & 0.08035529 \\
0.98493438 & 0.66894606 & 0.08214320 \\
0.16839084 & 0.17770227 & 0.24802023 \\
0.99458881 & 0.34178403 & 0.25169374 \\
0.34744412 & 0.99720380 & 0.25028888 \\
0.64786889 & 0.14841623 & 0.25407214 \\
0.50673174 & 0.34209577 & 0.25152875 \\
0.83449062 & 0.99269899 & 0.24481076 \\
0.14408093 & 0.65284723 & 0.25226292 \\
0.00724773 & 0.85172926 & 0.25191655 \\
0.34671855 & 0.49910010 & 0.25248096 \\
0.65074106 & 0.64884806 & 0.25039464 \\
0.50652878 & 0.84449376 & 0.23423627 \\
0.85523385 & 0.50473640 & 0.24865657 \\
0.01394547 & 0.17816073 & 0.41197313 \\
0.31988758 & 0.32828820 & 0.41564378 \\
0.17188649 & 0.47987065 & 0.42098793 \\
0.51205427 & 0.17942303 & 0.41865732 \\
0.83152643 & 0.33324071 & 0.41783250 \\
0.65539294 & 0.48691414 & 0.40840506 \\
0.00758801 & 0.67666599 & 0.42612446 \\
0.32884488 & 0.83664376 & 0.41466664 \\
0.16491472 & 0.98778288 & 0.41408233 \\
0.51100012 & 0.68388401 & 0.41266895 \\
0.82089961 & 0.84040963 & 0.40848901 \\
0.67403284 & 0.98440929 & 0.42162974 \\
0.47729570 & 0.31155265 & 0.57802518 \\
0.18241435 & 0.15266052 & 0.58237863 \\
0.33937350 & 0.00219965 & 0.58427085 \\
0.97916345 & 0.30690037 & 0.58505948 \\
0.68494960 & 0.15838008 & 0.58228744 \\
0.8785858 & 0.00085236 & 0.58827404 \\
\hline & 0.81972890 & 0.58495528 \\
\hline & 0.66283650 & 0.57949542 \\
\hline & 0.81321826 & 0.58381946
\end{tabular}




\begin{tabular}{lll}
0.68028497 & 0.65960211 & 0.57415552 \\
0.83775735 & 0.50787549 & 0.57506078 \\
0.34438577 & 0.34191569 & 0.76040807 \\
0.14390445 & 0.99281638 & 0.75226130 \\
0.00330788 & 0.15953084 & 0.75009425 \\
0.85095853 & 0.34985754 & 0.74203775 \\
0.65995154 & 0.01066800 & 0.75564864 \\
0.48918933 & 0.15820352 & 0.75303592 \\
0.36443525 & 0.84791243 & 0.75768304 \\
0.14542151 & 0.50239056 & 0.74890539 \\
0.01373458 & 0.66629562 & 0.75150795 \\
0.85388487 & 0.85228208 & 0.75363534 \\
0.65062905 & 0.50123613 & 0.75295500 \\
0.49857359 & 0.64953862 & 0.75071622 \\
0.31736825 & 0.49603671 & 0.91854730 \\
0.16653835 & 0.18780341 & 0.92723394 \\
0.00597791 & 0.32638257 & 0.91631957 \\
0.81722393 & 0.48332412 & 0.91048388 \\
0.66708088 & 0.19244196 & 0.91770586 \\
0.51647603 & 0.33920947 & 0.91913804 \\
0.32416644 & 0.99834041 & 0.90658325 \\
0.15718370 & 0.68164721 & 0.92151002 \\
0.01388816 & 0.83751236 & 0.91718363 \\
0.80715592 & 0.97815141 & 0.91277408 \\
0.66002099 & 0.68084373 & 0.91751305 \\
0.52057164 & 0.84399061 & 0.91656770 \\
0.16182516 & 0.33834507 & 0.02556674 \\
0.66429706 & 0.32921934 & 0.03029565 \\
0.16641461 & 0.82899800 & 0.01802400 \\
0.66624464 & 0.82886234 & 0.02991390 \\
0.01273744 & 0.00748094 & 0.14680382 \\
0.50739780 & 0.00130189 & 0.14835787 \\
0.98952214 & 0.49686111 & 0.14945671 \\
0.49937372 & 0.49286306 & 0.14601124 \\
0.33663333 & 0.17539711 & 0.18091576 \\
0.83979464 & 0.16999023 & 0.18690439 \\
0.32630881 & 0.65723751 & 0.17918449 \\
0.84444063 & 0.68942157 & 0.18446607 \\
0.16701062 & 0.33855958 & 0.31284747 \\
0.67765494 & 0.33852540 & 0.30967679 \\
\hline & 0.83890448 & 0.30785866 \\
\hline & 0.83731739 & 0.30322269 \\
0.9967 & 0.00927592 & 0.36730811
\end{tabular}




$\begin{array}{lll}0.01156592 & 0.51098049 & 0.35855429 \\ 0.49786138 & 0.50220995 & 0.34977951 \\ 0.32882887 & 0.16259290 & 0.47720629 \\ 0.84543341 & 0.16566915 & 0.48278711 \\ 0.33815383 & 0.67701639 & 0.47222080 \\ 0.82407853 & 0.66571186 & 0.46961626 \\ 0.16466434 & 0.32136692 & 0.52483330 \\ 0.66708599 & 0.32304786 & 0.52156411 \\ 0.15874815 & 0.83049642 & 0.52623766 \\ 0.67231463 & 0.83174435 & 0.51580003 \\ 0.98617664 & 0.98874198 & 0.65263410 \\ 0.50204272 & 0.99538852 & 0.64811202 \\ 0.00931864 & 0.50484762 & 0.64105648 \\ 0.48889538 & 0.48314595 & 0.64303768 \\ 0.32271011 & 0.15818980 & 0.69180940 \\ 0.83598772 & 0.17090847 & 0.69065145 \\ 0.33998846 & 0.66976197 & 0.68778537 \\ 0.83289662 & 0.66099785 & 0.67943295 \\ 0.16207179 & 0.32732330 & 0.81621175 \\ 0.65904259 & 0.33306100 & 0.80729762 \\ 0.17622234 & 0.84259765 & 0.81520070 \\ 0.65746385 & 0.83499400 & 0.81149011 \\ 0.99516904 & 0.99788632 & 0.85938261 \\ 0.50418579 & 0.00484509 & 0.85638606 \\ 0.99563853 & 0.50911334 & 0.85166563 \\ 0.50514839 & 0.49585556 & 0.85620136 \\ 0.32930029 & 0.16343685 & 0.97648949 \\ 0.82874563 & 0.16607512 & 0.98239958 \\ 0.33349991 & 0.67114370 & 0.98127828 \\ 0.82595573 & 0.66171946 & 0.97733797\end{array}$

Snapshot: 40 ps (-818.801 eV)

$\begin{array}{lll}0.16256460 & 0.99548428 & 0.08035444 \\ 0.34585574 & 0.31542915 & 0.08532152 \\ 0.49498229 & 0.15836491 & 0.07179434 \\ 0.68330176 & 0.00883887 & 0.09413540 \\ 0.84891497 & 0.32007437 & 0.07501609 \\ 0.98626633 & 0.15974458 & 0.08937946 \\ 0.18549801 & 0.51021717 & 0.09264822 \\ 0.34588412 & 0.82528301 & 0.07596921 \\ 0.49583083 & 0.66995100 & 0.07720177 \\ 0.67817741 & 0.50591263 & 0.08402754 \\ 0.84261577 & 0.82544503 & 0.07721141 \\ 0.98768026 & 0.65929195 & 0.08019372\end{array}$




\begin{tabular}{lll}
0.15046452 & 0.15490546 & 0.25397021 \\
0.00036028 & 0.35042393 & 0.25377657 \\
0.33942651 & 0.99138106 & 0.24911882 \\
0.65510112 & 0.15923834 & 0.25846057 \\
0.50701469 & 0.36059810 & 0.25224073 \\
0.84000380 & 0.99617631 & 0.25274611 \\
0.16042908 & 0.66212065 & 0.24546328 \\
0.00185351 & 0.85381036 & 0.25186438 \\
0.35356756 & 0.50307444 & 0.25101348 \\
0.66026972 & 0.66408901 & 0.25330145 \\
0.49824485 & 0.84764375 & 0.25604623 \\
0.84921513 & 0.50531677 & 0.24690109 \\
0.02129507 & 0.19519494 & 0.41253257 \\
0.32766447 & 0.33767100 & 0.41741736 \\
0.16874589 & 0.48847596 & 0.41693639 \\
0.51111429 & 0.18866917 & 0.41442720 \\
0.81310072 & 0.33787447 & 0.41152989 \\
0.66284444 & 0.48771123 & 0.41679738 \\
0.00123602 & 0.66242041 & 0.41647038 \\
0.31957433 & 0.84091483 & 0.42327937 \\
0.15768092 & 0.98117173 & 0.42562059 \\
0.51183325 & 0.68826070 & 0.42771050 \\
0.81790780 & 0.84172812 & 0.41591122 \\
0.65837675 & 0.98843010 & 0.41847464 \\
0.48870554 & 0.31889511 & 0.58139564 \\
0.16595269 & 0.16841997 & 0.58127046 \\
0.34116095 & 0.01375717 & 0.58198193 \\
0.98592322 & 0.32113992 & 0.58794616 \\
0.67817566 & 0.16859098 & 0.57860943 \\
0.82401022 & 0.01878281 & 0.58097893 \\
0.48756541 & 0.82104366 & 0.58907768 \\
0.17297679 & 0.66056256 & 0.57830687 \\
0.33120899 & 0.50333377 & 0.58707468 \\
0.98733255 & 0.82666278 & 0.57967949 \\
0.68276113 & 0.67353784 & 0.58739255 \\
0.83102363 & 0.52119166 & 0.58712688 \\
0.34757491 & 0.34605549 & 0.74994899 \\
0.14689617 & 0.99657398 & 0.74427024 \\
0.99267579 & 0.13976324 & 0.75103941 \\
0.85266294 & 0.35223304 & 0.74301045 \\
0.16276149 & 0.49785315 & 0.73898122 \\
\hline & 0.00102068 & 0.74665736 \\
\hline
\end{tabular}




\begin{tabular}{lll}
0.00152226 & 0.64748816 & 0.74851637 \\
0.85028886 & 0.84121783 & 0.75493771 \\
0.64397997 & 0.48214249 & 0.74985578 \\
0.51002586 & 0.65298190 & 0.75348615 \\
0.32323911 & 0.49280731 & 0.91182482 \\
0.15729077 & 0.17246312 & 0.91563754 \\
0.02216716 & 0.33949942 & 0.92420892 \\
0.81780130 & 0.48751019 & 0.91521890 \\
0.66814838 & 0.16427128 & 0.90459655 \\
0.50449614 & 0.32787744 & 0.92022234 \\
0.32063363 & 0.97859182 & 0.91808765 \\
0.17635483 & 0.68820022 & 0.91214857 \\
0.02317248 & 0.83859152 & 0.91766579 \\
0.82096336 & 0.99648230 & 0.91756156 \\
0.66952305 & 0.67870506 & 0.91515868 \\
0.53641808 & 0.84643455 & 0.91343763 \\
0.16943049 & 0.32758889 & 0.02966924 \\
0.66978176 & 0.33347665 & 0.01725745 \\
0.16133604 & 0.82052649 & 0.01620338 \\
0.67394617 & 0.84484386 & 0.02158500 \\
0.01002786 & 0.00930117 & 0.15513622 \\
0.50491319 & 0.01096246 & 0.14724122 \\
0.00620535 & 0.49596043 & 0.14883016 \\
0.50626303 & 0.49866732 & 0.15063802 \\
0.33289992 & 0.15951784 & 0.18801603 \\
0.83602473 & 0.16510534 & 0.18656305 \\
0.34864177 & 0.67320023 & 0.18674968 \\
0.83573417 & 0.67252162 & 0.18740459 \\
0.17621473 & 0.33366256 & 0.31358436 \\
0.66274428 & 0.34191451 & 0.30537537 \\
0.15558762 & 0.82710321 & 0.31187778 \\
0.66471862 & 0.83778391 & 0.32587226 \\
0.99697085 & 0.00352157 & 0.35431967 \\
0.49062781 & 0.99930385 & 0.35268690 \\
0.00768625 & 0.50239962 & 0.35199463 \\
0.49640795 & 0.50904629 & 0.35781287 \\
0.33105772 & 0.16759835 & 0.47760824 \\
0.82289786 & 0.16976442 & 0.47285582 \\
0.32967911 & 0.67592200 & 0.48688927 \\
0.63387053 & 0.66503356 & 0.47800169 \\
& 0.33705118 & 0.51870801 \\
\hline
\end{tabular}




$\begin{array}{lll}0.98476720 & 0.99187680 & 0.64816888 \\ 0.51163219 & 0.01124098 & 0.64334547 \\ 0.99328310 & 0.50477112 & 0.63998834 \\ 0.50003993 & 0.49576783 & 0.64449597 \\ 0.32310118 & 0.16231888 & 0.68593455 \\ 0.83873776 & 0.16728440 & 0.68436264 \\ 0.33915709 & 0.66723003 & 0.68601555 \\ 0.82263037 & 0.65244266 & 0.69405622 \\ 0.16856513 & 0.33147995 & 0.81100235 \\ 0.66297922 & 0.32115680 & 0.81165291 \\ 0.16535793 & 0.83261686 & 0.81076532 \\ 0.67068967 & 0.83064777 & 0.80915780 \\ 0.99773188 & 0.99841418 & 0.85668114 \\ 0.50192312 & 0.98812211 & 0.85254091 \\ 0.99339447 & 0.48933681 & 0.85534549 \\ 0.50038830 & 0.50050664 & 0.85193605 \\ 0.31927744 & 0.14505387 & 0.98093867 \\ 0.83702893 & 0.16817133 & 0.97429733 \\ 0.34121038 & 0.66897944 & 0.97816365 \\ 0.82929814 & 0.67149507 & 0.97685701\end{array}$

\section{(2) $\mathrm{Si} @ E P-\mathrm{Fe}_{2} \mathrm{O}_{3}$}

(2.1) $2 \times 2 \times 1$ supercell with the EP localized on Fe1 $(-829.858 \mathrm{eV})$

$\begin{array}{ccc}10.1413002014000000 & 0.0000000000000000 & 0.0000000000000000 \\ -5.0706501007000000 & 8.7826236017999992 & 0.0000000000000000 \\ 0.0000000000000000 & 0.0000000000000000 & 13.9027004242000007\end{array}$

$\begin{array}{lcc}\mathrm{O} & \mathrm{Fe} & \mathrm{Si} \\ 72 & 47 & 1\end{array}$

0.1852597094262997

0.3324235780448301

0.4874127121802198

$0.0163100845483613 \quad 0.0850694260350203$

0.6804381314677047

$0.3197457251547390 \quad 0.0842269381227621$

$\begin{array}{ll}0.1674006641282402 & 0.0844637035981047\end{array}$

0.8345126560043925

$0.0129713147209025 \quad 0.0843166417247695$

0.9842134267051003

$0.3222669409531168 \quad 0.0858925866834335$

0.1796393556952068

$0.1742717440001440 \quad 0.0824769107959521$

0.3335623519170481

$\begin{array}{lll}0.5146913700196987 & 0.0839090020926492\end{array}$

0.4830621704847896

$0.8194440412252807 \quad 0.0844222164454812$

0.6788741452968879

$0.6627739334316222 \quad 0.0857586609344587$

0.8221001582668690

$0.5090208928575554 \quad 0.0830729876144729$

0.9870668214175093

$0.8065231084279444 \quad 0.0820357530872148$

0.1609262305293208

$0.6690029137840428 \quad 0.0834114314414733$

0.0172803931496926

$0.1780515570189322 \quad 0.2606859907112975$

0.3470689353999330

$\begin{array}{lll}0.3431062874581790 & 0.2589295622242428\end{array}$

0.6561877735764262

$0.0024447267617426 \quad 0.2512297207044198$

$\begin{array}{lll}0.1530950670743975 & 0.2503867406229499\end{array}$ 


\begin{tabular}{|c|c|c|}
\hline 8487 & 0.3479 & \\
\hline 3835 & 17 & 0.2499 \\
\hline 83261370685 & 0.6526721878486135 & 0.249 \\
\hline 3 & 102 & 68425 \\
\hline 8 & 62 & 政 \\
\hline 2889 & 31768 & 4183 \\
\hline 4975598 & 0.8444 & 4150 \\
\hline & & \\
\hline 04 & $0.1 \mathrm{c}$ & 438 \\
\hline 99557 & 0.333 & 0805 \\
\hline 7 & 93 & \\
\hline & 0.18 & \\
\hline 57 & 0159 & 3993 \\
\hline 024 & 0.4866 & 4717 \\
\hline & & \\
\hline & 0 . & \\
\hline 015 & 0.98604 & 3058 \\
\hline 74 & 0.680 & 0.4 \\
\hline 6 & & \\
\hline 0.667 & 0.98 & 014 \\
\hline 999 & 0.32 & 407 \\
\hline 6 & 0.1 & 37 \\
\hline & & \\
\hline 97 & 56 & 7732 \\
\hline 0.6804645062232941 & 0.16680 & 0810 \\
\hline o & 0. & \\
\hline & & \\
\hline 4236 & 0.664 & 0.58 \\
\hline 0.334075351 & 0.5137915085522401 & 0.58 \\
\hline 9866791644459112 & 0.819421 & 069 \\
\hline & 0.66740939 & \\
\hline 0.8325004186712093 & 0.5132675650452145 & $0.583015 €$ \\
\hline 3463042674428694 & 0.3466687723687629 & 791985 \\
\hline & 0.00 & \\
\hline 0.0001002203459345 & 0.1531533621953271 & 0.7495679646909822 \\
\hline 8466775244241891 & 0.3468348939769526 & 0.7493719140091248 \\
\hline & $03<-$ & 0.74 \\
\hline 0.5003661321747044 & 0.1532957151415706 & 0.7491901638635408 \\
\hline 0.3466532805426753 & 0.8470143721539104 & 0.7492267210280978 \\
\hline 1530401762720148 & 0.5000054252570589 & 0.7492207964649508 \\
\hline 0004467124429329 & 0.6535285143017134 & 0.7494572718915730 \\
\hline 0.8467685137661798 & 0.8470559565238852 & 0.7495751757425139 \\
\hline 10 & 0.499 & 0.749226 \\
\hline 0.4997363231869868 & 0.6530291423617062 & 0.7492484288531971 \\
\hline
\end{tabular}




\begin{tabular}{|c|c|c|}
\hline & & \\
\hline 6 & 804177908 & 0 \\
\hline 41 & 153 & \\
\hline 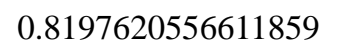 & 37 & \\
\hline & & \\
\hline & 740 & \\
\hline 87 & 618 & \\
\hline & & \\
\hline & & \\
\hline 85 & 0.98 & \\
\hline & & \\
\hline & & \\
\hline 581 & 036 & \\
\hline 0.66 & 842 & \\
\hline & & \\
\hline & & \\
\hline 1273 & 32 & 0 \\
\hline & 373 & \\
\hline & & \\
\hline 0.5 & 59 & \\
\hline 0.32 & 310 & \\
\hline 0.8 & & \\
\hline & & \\
\hline & 11 & 0.1 \\
\hline 0.6 & 0.33 & 0.3 \\
\hline & & \\
\hline & & \\
\hline 0.00 & 0.0 & \\
\hline 0.500 & 07 & 070 \\
\hline 3 & 0.49 & \\
\hline & & \\
\hline 0.331 & 0.16 & 0.478 \\
\hline 0.834 & 91 & 0.4 \\
\hline & & \\
\hline & 52 & 0.478 \\
\hline 0.16677 & 0.333 & 0.52 \\
\hline 0.004 & 47 & \\
\hline & & 0.52 \\
\hline 0.6682 & 0.8345977 & 0.520 \\
\hline 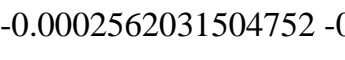 & 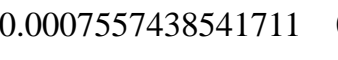 & 0.64641328400786 \\
\hline & 0001322283835436 & 0.64560839835 \\
\hline-0.0006450950374784 & 0.5002182613243188 & 0.6460147814989758 \\
\hline & & \\
\hline 0.3334473407703586 & 0.1666238346382275 & 0.6871309050945629 \\
\hline
\end{tabular}




$\begin{array}{rll}0.8331596386133476 & 0.1666156444199299 & 0.6870612005921588 \\ 0.3332904241981375 & 0.6668271613070692 & 0.6870415703713827 \\ 0.8331737964518510 & 0.6665477804015076 & 0.6871722212084670 \\ 0.1661996997978152 & 0.3334351474792982 & 0.8131082374223362 \\ 0.6666790346455906 & 0.3333341035754428 & 0.8131097356115672 \\ 0.1667947695619371 & 0.8336275637885986 & 0.8131534538762819 \\ 0.6663246095516279 & 0.8326880561651469 & 0.8130817023394734 \\ -0.0000138002985834 & 0.0003051233616631 & 0.8547469773156974 \\ 0.5003804878043511 & 0.0000308672801681 & 0.8541879511380644 \\ 0.000069895226920 & 0.5004675326937548 & 0.8539227211916349 \\ 0.4984486293474411 & 0.4986376677811525 & 0.8537989721317238 \\ 0.3336192221166527 & 0.1669933269960238 & 0.9805582029094255 \\ 0.8346024125185235 & 0.1663515414030890 & 0.9802197888600864 \\ 0.3323698045148429 & 0.6665263004212161 & 0.9800257412582107 \\ 0.8313658329457239 & 0.6662378518085499 & 0.9788651543396157 \\ 0.1675931087137136 & 0.3345558339641834 & 0.3211092952610840\end{array}$

(2.2) $2 \times 2 \times 1$ supercell with the EP localized on Fe2 $(-829.732 \mathrm{eV})$

$\begin{array}{ccc}0.1788926717372877 & 0.0148370954621372 & 0.0857429448344644 \\ 0.3320759315534861 & 0.3217893941193545 & 0.0837728172104332 \\ 0.4834178944338581 & 0.1745288349180456 & 0.0830920560171833 \\ 0.6868437111641242 & 0.0146846038949891 & 0.0843051451789530 \\ 0.8351088449212078 & 0.3205335258297259 & 0.0860962889076006 \\ 0.9878437123035072 & 0.1671170147647315 & 0.0838144295736363 \\ 0.1775745839285222 & 0.5098452028086258 & 0.0835352211598035 \\ 0.3230552192311932 & 0.8081022181513182 & 0.0827402112023860 \\ 0.4849653585537412 & 0.6648036584233866 & 0.0860146570353275 \\ 0.6810332535902933 & 0.5146099786279454 & 0.0835029390406491 \\ 0.8326877915413283 & 0.8188086590246095 & 0.0835228146275099 \\ 0.9849239389270704 & 0.6669005108633981 & 0.0832178157285073 \\ 0.1576539106581026 & 0.1738577032325955 & 0.2578464237026534 \\ 0.0172571565663180 & 0.3429684691320785 & 0.2581710766059687 \\ 0.3460806036412928 & 0.0021031386626194 & 0.2511348042087593 \\ 0.6620323334929644 & 0.1579319793586105 & 0.2544767844795756 \\ 0.5016192338251034 & 0.3487803096383202 & 0.2499719244068078 \\ 0.8475338602923139 & -0.0013638221460999 & 0.2500528035227539 \\ 0.1511282415100708 & 0.6528052758227725 & 0.2498321398169942 \\ 0.0017807932319621 & 0.8511449204995560 & 0.2496419646875552 \\ 0.3263356005116878 & 0.4831676248263336 & 0.2578863400042570 \\ 0.6492861867578305 & 0.6502370900424401 & 0.2498875081429141 \\ 0.4965558251136197 & 0.8386274411173944 & 0.2549706119724734 \\ 0.8500775500127710 & 0.4991430995308506 & 0.2497492170828889 \\ 0.0226512019672980 & 0.1912073559658038 & 0.4097877602463676 \\ 0.3089680602711902 & 0.3311875665481946 & 0.4097935225622190\end{array}$




\begin{tabular}{|c|c|c|}
\hline 572 & 42008 & 0. \\
\hline 0.5129297038411804 & 0.1804775677419652 & 0.4161512927929983 \\
\hline 8214842945813194 & 3358596271789333 & 0.4177399778727053 \\
\hline 6656390559691115 & 0.4865913235771747 & 144940405498 \\
\hline 0138826402859314 & 0.6793904334380950 & 0.4164543542728754 \\
\hline ).3198020926126961 & 0.8328107376148346 & 0.4162256669333306 \\
\hline 7787746629734 & 0.9859969239767898 & 0.41 \\
\hline 0400 & 36847158 & 0.41 \\
\hline 94280933975 & 0.8346235688135372 & 4108 \\
\hline 0.6682508588060742 & 0.9871360595148283 & 0.4167401519172349 \\
\hline 40915 & 39215355 & 0.581 \\
\hline 1796687662804474 & 0.1660658487333596 & 0.5821752782349018 \\
\hline 0.3333944843039959 & 0.0138344142569924 & 0.5825143961551070 \\
\hline 0.9866071063383337 & 0.3203695 & 0.582 \\
\hline 7403 & 0.166 & 0.582 \\
\hline 0.8361678345407703 & 0.016046 & 1613 \\
\hline 0.4862112961115926 & 0.8196570302682403 & 0.5825779570173504 \\
\hline 1799637083875602 & 0.6639894142228991 & 61835 \\
\hline 75 & 0. & \\
\hline 0.9869209149943838 & 0.8194594730974503 & 0.583112532 \\
\hline .6809077074713125 & 0.6675133983573195 & 0.5833584232425340 \\
\hline 377539 & 0.5133 & 0.583 \\
\hline .34663205548 & 30024890228 & 0.74 \\
\hline 0.1533364637716872 & 0.0000586353451849 & 0.7495925772587387 \\
\hline 0.0001419232903556 & 0.1532145946983825 & 0.7493061158275648 \\
\hline 8463682054607820 & 0.3465112738211230 & 0.7495765 \\
\hline 0.653060183083201 & -0.00009467 & 0.7496382299402624 \\
\hline 0.5001021003012074 & 0.1532429295894600 & 0.7495638236805463 \\
\hline 0.3467295820322129 & 0.8468974630820624 & 0.7494628809626622 \\
\hline 32 & 0.4996178 & 37356308917 \\
\hline .0001371389771356 & 0.6530567573765035 & 0.7491578580741405 \\
\hline 0.8467555703737755 & 0.8472278704346233 & 0.7494641389802174 \\
\hline .6527579732742496 & 0.4997966243887136 & 0.7493592249463805 \\
\hline 4999661534303764 & 0.6535212388041460 & 0.7496245420356501 \\
\hline 0.3192486114344260 & 0.4865614301466094 & 0.9164031651183936 \\
\hline 0.1667935018195739 & 0.1803335075357039 & 0.9164905862099226 \\
\hline 0.0125964990129143 & 0.3324876207572348 & 0.9164209482603388 \\
\hline 0.8197712807511836 & 0.4863190608223523 & 0.9165857800658522 \\
\hline 0.6662983337928250 & 0.1800695894311941 & 0.9177782859113042 \\
\hline 0.5141348155182125 & 0.3345348789430064 & 0.9157851756576131 \\
\hline 0.3195320009662316 & 0.9863644569039426 & 0.9173203338017616 \\
\hline 0.1650865480329478 & 0.6796005998028152 & 0.9154228150000649 \\
\hline .0131217858261882 & 0.8335283732315690 & 0.9164563183140778 \\
\hline .81969463972674 & 0.985941738865482 & 0.916405246783159 \\
\hline
\end{tabular}




\begin{tabular}{|c|c|c|}
\hline & & \\
\hline 3591 & 83318748052 & 0.917639 \\
\hline 54 & 36 & \\
\hline 066 & 5 & \\
\hline & & \\
\hline & & \\
\hline 00 & 194 & 0.14 \\
\hline 0.5006 & & \\
\hline & & \\
\hline 61 & 590 & 0 . \\
\hline & & \\
\hline & & \\
\hline & & \\
\hline 0.83 & 0.66 & 0 . \\
\hline 9 & 59 & \\
\hline & & \\
\hline 0.66 & 0.8 & \\
\hline 529 & 0.00 & \\
\hline 0.50 & 0.00 & \\
\hline & & \\
\hline 73 & & \\
\hline 0.33 & 0.1 & \\
\hline & & \\
\hline 53 & & \\
\hline 0.83 & 0.66 & \\
\hline 22 & 0.33 & 69 \\
\hline 0.66 & & \\
\hline 0.16 & 7723 & \\
\hline 0.66 & 7771617453 & 0.52 \\
\hline 0.00 & 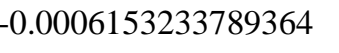 & \\
\hline & & \\
\hline 0.000 & 0.5 & 0.6 \\
\hline 399 & 702 & 34 \\
\hline & 43 & \\
\hline & & \\
\hline 0.33 & 8 & 64 \\
\hline 801 & 799 & 0.6 \\
\hline & 12 & \\
\hline & & \\
\hline 0.166 & 0.8328 & 0.812 \\
\hline & & \\
\hline 0.0004271759866163 & 0.0005547131493686 & 0.8540493944605820 \\
\hline 0.499 & & 0.8549 \\
\hline 0007614101764191 & 0.4988865415169609 & \\
\hline
\end{tabular}




$\begin{array}{lll}0.4992820091972399 & 0.5003750270136949 & 0.8538679779344212 \\ 0.3352721825941553 & 0.1685377967744310 & 0.9800108309572361 \\ 0.8329494605855727 & 0.1651894918421530 & 0.9806319396100515 \\ 0.3303494759924157 & 0.6661885901577856 & 0.9800558086271531 \\ 0.8329881972435114 & 0.6664094812559148 & 0.9785222277022496 \\ 0.1673884462804165 & 0.3334298779322323 & 0.3191278826427081\end{array}$

(2.3) $2 \times 2 \times 1$ supercell with the EP localized on Fe3 $(-829.858 \mathrm{eV})$

$\begin{array}{lll}0.1797114201418048 & 0.0169367750822984 & 0.0857589161566067 \\ 0.3350524116185986 & 0.3203603419254974 & 0.0839087471534259 \\ 0.4858816850719436 & 0.1664372007170923 & 0.0844219171674507 \\ 0.6799877402670635 & 0.0125870347423779 & 0.0844631249480857 \\ 0.8310519672437329 & 0.3147406153512771 & 0.0850684492691136 \\ 0.9873221890468821 & 0.1675753163701961 & 0.0842265503299077 \\ 0.1900570968992147 & 0.5157844510900138 & 0.0824772203041582 \\ 0.3325330847491346 & 0.8195607633168035 & 0.0843169726635443 \\ 0.4877536734494661 & 0.6654866733000966 & 0.0858927738375791 \\ 0.6819361949926628 & 0.5129320538887988 & 0.0834118201256455 \\ 0.8301459568303537 & 0.8211251762093149 & 0.0830731222575563 \\ 0.9844221777494027 & 0.6778993441464882 & 0.0820366696399052 \\ 0.1580513179646742 & 0.1735167012767987 & 0.2589885285618598 \\ 0.0171260742989335 & 0.3390754950316580 & 0.2606863954738678 \\ 0.3469333950344967 & 0.0024411156776071 & 0.2509181186004612 \\ 0.6553769187133982 & 0.1529324418079856 & 0.2512292873267570 \\ 0.5005541633953137 & 0.3478821205593177 & 0.2498580398407043 \\ 0.8471431353725825 & -0.0007612608711892 & 0.2496195898664748 \\ 0.1519532812223580 & 0.6534779989956296 & 0.2499705971239222 \\ 0.0023611785599317 & 0.8522276859708441 & 0.2497197417162747 \\ 0.3258265366459121 & 0.4827204274721549 & 0.2589298197134689 \\ 0.6484830274945075 & 0.6503010155737876 & 0.2491128146691977 \\ 0.4969071687335386 & 0.8438131328650561 & 0.2503866296886267 \\ 0.8443826629773610 & 0.4998537610002534 & 0.2541464200676281 \\ 0.0229123757731902 & 0.1893904320436996 & 0.4115523581448192 \\ 0.3091944460941363 & 0.3313895499481192 & 0.4102137311945959 \\ 0.1682261684910377 & 0.4770244938451262 & 0.4104499923690233 \\ 0.5120628427880145 & 0.1797151915227078 & 0.4165869767121370 \\ 0.8197315284643313 & 0.3336867611261847 & 0.41668648559615643 \\ 0.6637300536599352 & 0.4856386697414132 & 0.4176833359942997 \\ 0.0141880222206568 & 0.6789823895511957 & 0.4163105607653688 \\ 0.3198782824744149 & 0.8321637326226776 & 0.4164106439304860 \\ 0.1658682514375866 & 0.9855256600137658 & 0.4166097404604641 \\ 0.5140580803896775 & 0.6802794611695793 & 0.4160996587545474 \\ 0.8203608186647182 & 0.8337037470634296 & 0.4159088078115723 \\ 0.6681648246734087 & 0.9872521810588881 & 0.4159526285011583\end{array}$




\begin{tabular}{|c|c|c|}
\hline & & \\
\hline 897027689 & 3 & 0.58244 \\
\hline 335370112 & 0134153358961893 & 5025 \\
\hline 9862282354353769 & 355456512 & 719 \\
\hline 0 & 98 & \\
\hline & & \\
\hline 02 & & 092 \\
\hline & & \\
\hline & & \\
\hline & & \\
\hline 150 & 484 & 0.58 \\
\hline & & \\
\hline & & \\
\hline 0.15 & 21 & \\
\hline 00 & & \\
\hline 45 & & \\
\hline & & \\
\hline 98 & 0.1 & \\
\hline 79 & 38 & \\
\hline & & \\
\hline 75 & 0.6 & \\
\hline 45 & 0.8 & \\
\hline 1 & 0.4 & \\
\hline & & \\
\hline & & \\
\hline 71 & & \\
\hline - & & \\
\hline & & \\
\hline 08 & 0.1 & \\
\hline 0.513 & 0.333 & 52 \\
\hline 832294 & 0.98 & \\
\hline & & \\
\hline 0.014 & 0.835 & 0.91 \\
\hline 0.8196 & 0.986 & 740 \\
\hline & & \\
\hline & & \\
\hline 0.1682832813816666 & 0.3347613315030038 & 25940289 \\
\hline & & \\
\hline & 0.835 & 0070 \\
\hline 0.66 & 0.8326002749 & 0.02 \\
\hline 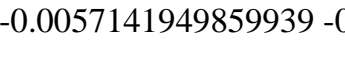 & 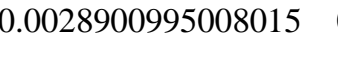 & 0.1441679728607390 \\
\hline 3028106 & .00101 & 0.1464516718931843 \\
\hline-0.0014 & 0.50200649 & 0.143 \\
\hline & 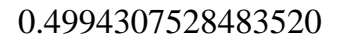 & \\
\hline
\end{tabular}




\begin{tabular}{cccc}
0.3312793974558942 & 0.1692822099278291 & 0.1889797738158374 \\
0.8390742853370960 & 0.1709470239195933 & 0.1894113289797764 \\
0.3272239910012557 & 0.6589280294392590 & 0.1881724451819399 \\
0.8371643975939732 & 0.6645676977649496 & 0.1870274915392331 \\
0.6655161176713184 & 0.3322206080431900 & 0.3134405752482013 \\
0.1674209382966503 & 0.8333042929738008 & 0.3124868971687123 \\
0.6656795683815130 & 0.8328293361324087 & 0.3113025093756152 \\
0.0003176793912297 & -0.0000835802969108 & 0.3526505341770962 \\
0.5001199278423780 & -0.0004305509816575 & 0.3534575827852995 \\
-0.0006364213615746 & 0.4984590311736902 & 0.3498952951303492 \\
0.4996763656107893 & 0.5003477274009061 & 0.3523478158550233 \\
0.3313783797453490 & 0.1649652075338358 & 0.4784285561649447 \\
0.8338630667458463 & 0.1683447744218362 & 0.4781616886000132 \\
0.3341521815414623 & 0.6656427850591397 & 0.4779872250856134 \\
0.8335418333297399 & 0.6662151900794190 & 0.4786499430336319 \\
0.1663705969685633 & 0.3332223784234333 & 0.5237102927001456 \\
0.6657779448159706 & 0.3334559919206221 & 0.5205652123484874 \\
0.1663148411070198 & 0.8317173002615733 & 0.5201625402726612 \\
0.6677704383795884 & 0.8340988666602664 & 0.5199216061508557 \\
-0.0002236049183810 & -0.0008394166304860 & 0.6459686898477843 \\
0.4999122370955247 & -0.0002196098201009 & 0.6456088329258145 \\
-0.0004995025769197 & 0.5002568999708273 & 0.6464133514442192 \\
0.5008640057910998 & 0.5006454227022237 & 0.6460145664541276 \\
0.3335358250818015 & 0.1667082364309717 & 0.6870414177025740 \\
0.8331753586457906 & 0.1665519606513035 & 0.6871309290822063 \\
0.3334559104742611 & 0.6668405608322233 & 0.6870614556244345 \\
0.8333734800172453 & 0.6668255180229572 & 0.6871726328648782 \\
0.1672351348895870 & 0.3337993788104895 & 0.8131084452208636 \\
0.6668313310105983 & 0.3332042424469110 & 0.8131527900408990 \\
0.1663623503379601 & 0.8336739889803153 & 0.8130822715087573 \\
0.6666546186107392 & 0.8333199303697318 & 0.8131096003249667 \\
0.0001882767392160 & 0.0015499191198423 & 0.8537988785660399 \\
0.4996497186706393 & -0.0003815467287974 & 0.8541873112504281 \\
0.0003175795492908 & 0.5000118656538591 & 0.8547469517670264 \\
0.5003972196528547 & 0.4999286826596521 & 0.8539225699588910 \\
0.3341567343116136 & 0.1676291323656181 & 0.9800260399148785 \\
0.8333742732378226 & 0.1663794837519554 & 0.9805581452659063 \\
0.3317477356281003 & 0.6653952078975212 & 0.9802200231018189 \\
0.8348708168670133 & 0.6686328870432408 & 0.9788653527233586 \\
\hline
\end{tabular}

(2.4) $2 \times 2 \times 1$ supercell with the EP localized on Fe4 $(-829.858 \mathrm{eV})$

$0.1777339766124231 \quad 0.0122466224558854 \quad 0.0858927076448226$

$\begin{array}{llll}0.3257248438889039 & 0.3099435403607962 & 0.0824770836978349\end{array}$ 


\begin{tabular}{llll}
0.4870293052070989 & 0.1674704952116317 & 0.0843194632833197 \\
0.6805539900557678 & 0.0141141548087494 & 0.0844221639152072 \\
0.8372253190903656 & 0.3202867844864270 & 0.0857601711880019 \\
0.9853060158143340 & 0.1649456564010184 & 0.0839091357572817 \\
0.1802553083969281 & 0.5126770119058291 & 0.0842261701483787 \\
0.3326013086060583 & 0.8200108775343514 & 0.0844627362299732 \\
0.4836861036143519 & 0.6689405135342839 & 0.0850633723829511 \\
0.6934814233235628 & 0.5155763871555874 & 0.0820369929064207 \\
0.8309937705495725 & 0.8180626443395336 & 0.083410647505242 \\
0.9909854557785378 & 0.6698588638832176 & 0.0830712273635719 \\
0.1569003853857013 & 0.1741747667838184 & 0.2589347865356262 \\
0.0154635874061871 & 0.3419453245709964 & 0.2589929010328467 \\
0.3469030544236212 & 0.0030901624894362 & 0.2503838807193509 \\
0.6555080678825637 & 0.1530703667584246 & 0.2509167629631528 \\
0.5015206290390424 & 0.3480515548918438 & 0.2499745760963434 \\
0.8473288271267642 & -0.0005543451146121 & 0.2498592341277213 \\
0.1520982028678061 & 0.6528534843808964 & 0.2496184176891633 \\
0.0018171321364174 & 0.8515196157370071 & 0.2491110497305201 \\
0.3219499576232688 & 0.4828783030581876 & 0.2606930578325957 \\
0.6554650851084355 & 0.6556135123702109 & 0.2541486095898115 \\
0.4975562707094476 & 0.8446236017758292 & 0.2512272700571164 \\
0.8498711512362277 & 0.4976425989167501 & 0.2497187053520556 \\
0.0221961394775945 & 0.1908045988192852 & 0.4102163960169332 \\
0.3087984112752059 & 0.3317776786697423 & 0.4104554240115129 \\
0.1664763339386028 & 0.4770876013222011 & 0.4115549088659946 \\
0.5122807167271858 & 0.1801244873430951 & 0.4164090604896923 \\
0.8196566498066066 & 0.3341316642255372 & 0.4166081136589413 \\
0.6647975462580753 & 0.4858168996702039 & 0.4163107293563955 \\
0.0133419585222808 & 0.6796404783021601 & 0.4159060525536749 \\
0.3190873912201338 & 0.8318318725158707 & 0.4159508238435343 \\
0.1662211195367121 & 0.9859427308991280 & 0.4160988692967671 \\
0.5139529272497904 & 0.6802707320561743 & 0.4166894729579729 \\
0.8219056344610113 & 0.8362692490791702 & 0.4176833317859309 \\
0.6676529777105648 & 0.9879423419917673 & 0.4165864569322829 \\
0.4838741862305970 & 0.3200335069704758 & 0.5812938888265454 \\
0.179767093239244 & 0.1658125607487373 & 0.5822449958292312 \\
\hline
\end{tabular}




\begin{tabular}{|c|c|c|}
\hline 034 & 0 & \\
\hline 9280 & 134087453217301 & 82881 \\
\hline 732 & 674 & 4 \\
\hline & & \\
\hline 06 & 03371 & 56 \\
\hline 45 & 342 & 762 \\
\hline 6529 & 963 & 0.749 \\
\hline & & \\
\hline & & \\
\hline 0.15 & 0.4 & 0.7 \\
\hline 0.00 & & \\
\hline & & \\
\hline 929 & 0.4 & 0 . \\
\hline 6106 & 0.6 & 792 \\
\hline & & \\
\hline & & \\
\hline 0.01 & 0.3 & 119 \\
\hline 79 & 0.4 & \\
\hline & & \\
\hline 0.5 & 0.3 & 0 \\
\hline 269 & 0.9 & 0.9 \\
\hline 0.1 & & \\
\hline 0.0 & & \\
\hline 0.8 & 0.9 & 0.5 \\
\hline 0.66 & 0.67 & 0.9 \\
\hline & & \\
\hline & & \\
\hline 0.668 & 0.33 & 0.02 \\
\hline 0.168 & 0.83 & 726 \\
\hline 5448 & 0.8322978758930138 & 0.021513 \\
\hline & מחדרכרתרו & 14 \\
\hline 0.499 & 0.000 & 990 \\
\hline 0.0028 & 0.5 & 591 \\
\hline & & \\
\hline 4644010867 & 0.1727759962222096 & 0.188172 \\
\hline 0.8380068830087549 & 0.1687256552149073 & 0.1889807183709042 \\
\hline & & \\
\hline 89343496854 & 0.6628352048834 & 0.1870245923033743 \\
\hline 0.6658856131605569 & 0.3325838409199 & 0.31248355 \\
\hline 0.1671 & 0.8343172509256779 & 0.311 \\
\hline 386769 & 0.8344847047116496 & 0.3134395907631050 \\
\hline 0.0006 & 0.000326344082 & 0.35 \\
\hline & & 0.3 \\
\hline .0003997418863222 & 0.49968123041128 & 8950250 \\
\hline
\end{tabular}




$$
\begin{array}{clll}
0.4990937730331156 & 0.5006396905201793 & 0.3499019251253831 \\
0.3314856099148626 & 0.1658447793911927 & 0.4779881730808202 \\
0.8335854879054290 & 0.1686254366689528 & 0.4784298038004114 \\
0.3344804027527437 & 0.6661379842400612 & 0.4781635140204811 \\
0.8326746532408157 & 0.6664619229981125 & 0.4786492942364574 \\
0.1668509562502477 & 0.3336296098595534 & 0.5237146679829658 \\
0.6653990193070983 & 0.3336882627534248 & 0.5201604361846041 \\
0.1663272384859979 & 0.8322277542066954 & 0.5199183540422648 \\
0.6676788691854103 & 0.8342258034727330 & 0.5205652203473710 \\
-0.0002207924979276 & -0.0008660634800259 & 0.6460140818241680 \\
0.4998678833258672 & 0.0000898002985730 & 0.6456093066604363 \\
-0.0006160134726813 & 0.5002255657065847 & 0.6459681764833252 \\
0.5007552692497661 & 0.5004988404081988 & 0.6464123483985098 \\
0.333384710809911 & 0.1665412898534039 & 0.6870615421490344 \\
0.8331714835842250 & 0.1664637195881340 & 0.6870407558168736 \\
0.3333769884279039 & 0.6668253046871230 & 0.6871317813749401 \\
0.8334521387064299 & 0.6666248711990467 & 0.6871732288087966 \\
0.1665641003943395 & 0.3327636148894351 & 0.8131073735565648 \\
0.6673133159702636 & 0.3336376724650607 & 0.8130832817180734 \\
0.1666647256309376 & 0.8333448563228486 & 0.8131095844191816 \\
0.6663721226123558 & 0.8331670799619673 & 0.8131522419540328 \\
-0.0004673527331883 & -0.0003971558137299 & 0.8539223593015085 \\
0.4999687565407975 & 0.0003503314376027 & 0.8541867149909581 \\
0.0013636397302705 & 0.4998083762745241 & 0.8537993140495274 \\
0.499694422248867 & 0.4996812824938505 & 0.8547451391184804 \\
0.3336491837385435 & 0.1682557310578127 & 0.9802212063306123 \\
0.8334720796600154 & 0.1658398063319441 & 0.9800271339419822 \\
0.3330035399907898 & 0.6666199980492211 & 0.9805562697862402 \\
0.8337667677853688 & 0.6651289006642810 & 0.9788650086675044 \\
0.1654442201454835 & 0.3330346936483327 & 0.3211159799651672 \\
& &
\end{array}
$$

(2.5) $2 \times 2 \times 1$ supercell with the EP localized on Fe5 $(-829.880 \mathrm{eV})$
0.1749898735305866
$0.0105604745156309 \quad 0.0800605276942872$
0.3332100033989742
$0.3195756142277320 \quad 0.0828761675266668$
0.4905658668174342
$0.1647764132582518 \quad 0.0796784185607199$
0.6815766018853486
$0.0134041330779668 \quad 0.0844832756772432$
0.8367031555004855
$\begin{array}{ll}0.3205989047440753 & 0.0855672613467643\end{array}$
$\begin{array}{llll}0.9872909778186381 & 0.1672723914719966 & 0.0838499928468934\end{array}$
$\begin{array}{llll}0.1799592817457020 & 0.5130119513696290 & 0.0837162523619649\end{array}$
$\begin{array}{llll}0.3329668881635272 & 0.8180989804458945 & 0.0836872185133971\end{array}$
$\begin{array}{llll}0.4848487355809472 & 0.6627742969720790 & 0.0863493540176857\end{array}$
$\begin{array}{llll}0.6823610131805358 & 0.5135476748993186 & 0.0830278430014666\end{array}$
$\begin{array}{llll}0.8333371091922026 & 0.8194540425500975 & 0.0839986379272985\end{array}$
$\begin{array}{llll}0.9865916951910748 & 0.6675443963855138 & 0.0829959319826519\end{array}$ 


\begin{tabular}{|c|c|c|}
\hline 4 & 5 & \\
\hline 56 & 0.3440408832819210 & 37243 \\
\hline 3748509065767 & 9973472001437986 & גר11 \\
\hline 5 & 30 & 34 \\
\hline 40 & 69 & 003 \\
\hline 3444867249434427 & 30 & 0.2 \\
\hline & & \\
\hline & & \\
\hline & 0 . & \\
\hline 83230 & 0.65338524181 & 0.24 \\
\hline & & \\
\hline & & \\
\hline 53 & 0.19 & 744 \\
\hline 21 & 0.331 & 0.41 \\
\hline & & \\
\hline 04 & 0 . & 336 \\
\hline 152 & 0.3 & 2407 \\
\hline 4 & 0.4 & $0 .{ }^{2}$ \\
\hline & & \\
\hline 36 & 0.8 & \\
\hline 443 & 0.98 & 0. \\
\hline 5 & & \\
\hline & & \\
\hline 40 & 0.98 & \\
\hline 881 & 0.3 & 0.5 \\
\hline 5 & 0. & \\
\hline & & \\
\hline 283 & 9532 & 0.5 \\
\hline 46 & 0.1 & 0.58 \\
\hline & & \\
\hline & 0.81 & 0.58 \\
\hline 869 & 0.66 & 0.58 \\
\hline & & \\
\hline & & \\
\hline 6807572168112483 & 0.6677722283991480 & 0.5833112517736296 \\
\hline 326948814052703 & 0.5137097404324245 & 0.5832067574 \\
\hline & 0.34 & 0.74 \\
\hline 1533697438512192 & 0.0001439859832305 & 0.7493341074755745 \\
\hline 838 & 80002 & 395123471 \\
\hline 90 & 0.3469208998101743 & 0.74 \\
\hline 0766 & 00066128132846 & 0.7495138741703664 \\
\hline 5003284268712 & 0.1532081156856462 & 0.749131 \\
\hline 5 & 0.8468484884 & 0.7490 \\
\hline 1 & - & 0.14 \\
\hline
\end{tabular}




$$
\begin{aligned}
& \begin{array}{llll}
0.0005831169143855 & 0.6530764660752472 & 0.7492066219131897
\end{array} \\
& \begin{array}{llll}
0.8467641337253680 & 0.8470878485639379 & 0.7496796236978742
\end{array} \\
& \begin{array}{llll}
0.6529646796173090 & 0.4997632226298636 & 0.7496162401905163
\end{array} \\
& \begin{array}{llll}
0.5000693819799080 & 0.6531776403119066 & 0.7498555401349168
\end{array} \\
& \begin{array}{llll}
0.3200034307026711 & 0.4868024294097366 & 0.9165695715455966
\end{array} \\
& \begin{array}{llll}
0.1677497133071785 & 0.1806607066136907 & 0.9164927831295250
\end{array} \\
& \begin{array}{llll}
0.0136636552038263 & 0.3328289095971889 & 0.9166969122444578
\end{array} \\
& \begin{array}{llll}
0.8206630351158799 & 0.4864052557384571 & 0.9164094504179578
\end{array} \\
& \begin{array}{llll}
0.6690184166818127 & 0.1801746003582420 & 0.9157871764888017
\end{array} \\
& \begin{array}{llll}
0.5144173934799455 & 0.3331662579921782 & 0.9164990819098988
\end{array} \\
& \begin{array}{llll}
0.3198842430946613 & 0.9858536664855355 & 0.9159854588152133
\end{array} \\
& \begin{array}{llll}
0.1670355602178489 & 0.6797423858268776 & 0.9163276116917992
\end{array} \\
& \begin{array}{llll}
0.0128655382362559 & 0.8319941283404874 & 0.9151566546725944
\end{array} \\
& \begin{array}{llll}
0.8199940480468806 & 0.9854947444231956 & 0.9171876687061431
\end{array} \\
& \begin{array}{llll}
0.6659904775404448 & 0.6796583587359138 & 0.9172170747652477
\end{array} \\
& \begin{array}{llll}
0.5138045344832026 & 0.8331005501838654 & 0.9176115693072857
\end{array} \\
& \begin{array}{llll}
0.1676622800683614 & 0.3331341078386448 & 0.0206168426661912
\end{array} \\
& \begin{array}{llll}
0.6686923195693137 & 0.3318144810989057 & 0.0207360508327640
\end{array} \\
& \begin{array}{llll}
0.1677529838297739 & 0.8340454544835526 & 0.0199182460771219
\end{array} \\
& \begin{array}{llll}
0.6648688522324774 & 0.8315203717081574 & 0.0228443705748478
\end{array} \\
& \begin{array}{llll}
0.0028416070323035 & 0.0021617295866399 & 0.1452476074203749
\end{array} \\
& \begin{array}{llll}
0.4991012410454740 & 0.0010811115749304 & 0.1455454282456881
\end{array} \\
& \begin{array}{llll}
0.0023058012612841 & 0.5040687525225610 & 0.1448200169258067
\end{array} \\
& \begin{array}{llll}
0.5012370575709998 & 0.4931568040780266 & 0.1453669981422561
\end{array} \\
& \begin{array}{llll}
0.3257025225688929 & 0.1631793647146862 & 0.1901311593288169
\end{array} \\
& \begin{array}{llll}
0.8371159580576266 & 0.1673605541154771 & 0.1892933293834490
\end{array} \\
& \begin{array}{llll}
0.3318380203937113 & 0.6621470341033059 & 0.1891751644994369
\end{array} \\
& \begin{array}{llll}
0.8334042402998273 & 0.6688217945881076 & 0.1874427967266361
\end{array} \\
& \begin{array}{llll}
0.6656354958381205 & 0.3339243285141033 & 0.3115856052288953
\end{array} \\
& \begin{array}{llll}
0.1681155194607018 & 0.8344152392390836 & 0.3120944461847674
\end{array} \\
& \begin{array}{llll}
0.6658604152691250 & 0.8333250263517440 & 0.3127437809582130
\end{array} \\
& \begin{array}{llll}
0.0003865932117011 & 0.0033255130704383 & 0.3525588256089360
\end{array} \\
& 0.4995200409972951-0.0001115208015478 \quad 0.3528935850945848 \\
& \begin{array}{llll}
-0.0010369274586082 & 0.5001383100512730 & 0.3527414625696106
\end{array} \\
& \begin{array}{llll}
0.4992817051552298 & 0.4990042646084344 & 0.3524335418367287
\end{array} \\
& \begin{array}{llll}
0.3313855098894778 & 0.1658399029199179 & 0.4774864805202190
\end{array} \\
& \begin{array}{llll}
0.8324208862350821 & 0.1687699289502382 & 0.4787328121388341
\end{array} \\
& \begin{array}{llll}
0.3347536030694979 & 0.6660505458502975 & 0.4781337889360198
\end{array} \\
& \begin{array}{llll}
0.8336337338641009 & 0.6677425441520658 & 0.4791136817614263
\end{array} \\
& \begin{array}{llll}
0.1657851605296727 & 0.3329000178363098 & 0.5236455337515016
\end{array} \\
& \begin{array}{llll}
0.6656979818628909 & 0.3342955210361581 & 0.5199130300621473
\end{array} \\
& \begin{array}{llll}
0.1667658478231884 & 0.8324520343488707 & 0.5199500514075205
\end{array} \\
& \begin{array}{llll}
0.6678157903251493 & 0.8345903517917109 & 0.5201187172874452
\end{array} \\
& -0.0004052403695701-0.0007794259190554 \quad 0.6462110258000010
\end{aligned}
$$




$\begin{array}{cccc}0.4999149015757459 & -0.0000449261874546 & 0.6454233837252134 \\ -0.0006008687896210 & 0.5004453678723667 & 0.6461267981287591 \\ 0.5007698654578556 & 0.5007761084258043 & 0.6460968086170632 \\ 0.3335433797256011 & 0.1667354742897956 & 0.6867420022788746 \\ 0.8331875199537402 & 0.1665307090051468 & 0.6873392059461608 \\ 0.3334709368287065 & 0.6665142129450538 & 0.6870112452852327 \\ 0.8333729853629118 & 0.6667399106692939 & 0.6872231828517257 \\ 0.1669520310274434 & 0.3330651183788233 & 0.8129564019299608 \\ 0.6667048369350934 & 0.3331372494107263 & 0.8128478482020277 \\ 0.1669925529924207 & 0.8334344803308167 & 0.8123599846139543 \\ 0.6668456415050087 & 0.8330655469295685 & 0.8134923544133039 \\ 0.0008472969098746 & 0.0005379239263053 & 0.8543809137632774 \\ 0.5005329830840164 & -0.0001546477022634 & 0.8541592645125072 \\ 0.0005622515573460 & 0.4995156318303774 & 0.8539064756142306 \\ 0.4996945672988957 & 0.4991299277228050 & 0.8543143184721078 \\ 0.3350028960163721 & 0.1663054401926678 & 0.9819690389610712 \\ 0.8339820817318018 & 0.1653510874722732 & 0.9801641712504955 \\ 0.3330909123776841 & 0.6663871269196087 & 0.9803351385870656 \\ 0.8335521228117346 & 0.6666220958523733 & 0.9786455368139547 \\ 0.1643307367877172 & 0.3337671097801522 & 0.3204058505062150\end{array}$

(2.6) $2 \times 2 \times 1$ supercell with the EP localized on Fe6 $(-829.880 \mathrm{eV})$

$\begin{array}{lll}0.1779254710099554 & 0.0151515492531778 & 0.0863495474550737 \\ 0.3330528057992288 & 0.3200409521156660 & 0.0837163970698150 \\ 0.4851322107018072 & 0.1670334173382063 & 0.0836871322410241 \\ 0.6742108042479664 & 0.0094344145858947 & 0.0796784446736427 \\ 0.8355707626676611 & 0.3250103542220753 & 0.0800606360092510 \\ 0.9863657524931130 & 0.1667902381288480 & 0.0828761039239697 \\ 0.1799815560787743 & 0.5127091096194049 & 0.0838499619790252 \\ 0.3318276573315078 & 0.8184234767264043 & 0.0844832650049225 \\ 0.4838958883464290 & 0.6632967383916902 & 0.0855673258807789 \\ 0.6809531801723617 & 0.5134085691091909 & 0.0829957748434544 \\ 0.8311867105540630 & 0.8176391990583587 & 0.0830280482652942 \\ 0.9861172054908294 & 0.6666632778414976 & 0.0839985517760890 \\ 0.1588426342178686 & 0.1759066506765389 & 0.2583714946353186 \\ 0.0267501641389282 & 0.3523132880242434 & 0.2604473371109898 \\ 0.3452213867623742 & 0.0018131275874089 & 0.2506417380615457 \\ 0.6489720048370998 & 0.1516248697356139 & 0.2505728993464638 \\ 0.5009527757419346 & 0.3479549184514243 & 0.2493566714443570 \\ 0.8501662872989019 & 0.9933426258586074 & 0.2510116848706403 \\ 0.1539008482481710 & 0.6555132548001825 & 0.2500750631693189 \\ 0.0027117406615813 & 0.8493266240963989 & 0.2493956992894391 \\ 0.3298989549477955 & 0.4858581299932550 & 0.2587244316456660 \\ 0.6499283039571605 & 0.6497900599016321 & 0.2493657573535829\end{array}$




\begin{tabular}{cccc}
0.4985445023200375 & 0.8445944431994549 & 0.2502874134335090 \\
0.8499378769920405 & 0.4970287327911340 & 0.2492142641328896 \\
0.0235985480360880 & 0.1921741869841797 & 0.4103214912697764 \\
0.3101289556614891 & 0.3323823919517653 & 0.4099089815603735 \\
0.1717153751747032 & 0.4799514770628917 & 0.4122518271910863 \\
0.5124366623422056 & 0.1806971284460837 & 0.4165040805710629 \\
0.8203819829521251 & 0.3338662320043400 & 0.4157705316043053 \\
0.6662360065719493 & 0.4862828915058685 & 0.4162328599314349 \\
0.0143657318119159 & 0.6790699299623862 & 0.4165854313004344 \\
0.3204474518900015 & 0.8327263094251106 & 0.4165761185839392 \\
0.1660767837808274 & 0.9860136550834886 & 0.4164910642013819 \\
0.5153029939745605 & 0.6804558556944630 & 0.4166662059212298 \\
0.8211754204187670 & 0.8338897975771514 & 0.4171636985072645 \\
0.6687932776837343 & 0.9877658649979812 & 0.4156108352004306 \\
0.4841765161251100 & 0.3201614341061247 & 0.5810487646986555 \\
0.1796919321866526 & 0.1661801593538597 & 0.5823730824769003 \\
0.3332615394761015 & 0.0138169194017186 & 0.5824228137047334 \\
0.9865234504368369 & 0.3206812154189880 & 0.5824093934685007 \\
0.6805315899859589 & 0.1666219659909012 & 0.5820904064981940 \\
0.8364316657377414 & 0.0161231233231637 & 0.5810161371276351 \\
0.4871211125670408 & 0.8199589185589469 & 0.5825561573349213 \\
0.1804223719897396 & 0.6645549298233561 & 0.5817578436881438 \\
0.3345407234673087 & 0.5139584106993182 & 0.5829857105389190 \\
0.9870148791756725 & 0.8192425566383716 & 0.5833113063643278 \\
0.6810147517753957 & 0.6673049014534999 & 0.5832067920454901 \\
0.8327147414139171 & 0.5129920339141282 & 0.5830624730140264 \\
0.3463420025593639 & 0.3466246342372097 & 0.7494459092608660 \\
0.1531082955222004 & -0.0000694542602042 & 0.7498555455549583 \\
0.0000616929902496 & 0.1530456806516590 & 0.7493201186968120 \\
0.8467742102585623 & 0.3466301876442329 & 0.7493340271802077 \\
0.6528796412845946 & -0.0003283738838348 & 0.7491317638478926 \\
0.4998008964827921 & 0.1529522395674538 & 0.7490885916934783 \\
0.3463147382157566 & 0.8469760243693942 & 0.7495139728616536 \\
0.1530313098719658 & 0.4999339561388227 & 0.7496827305949719 \\
0.0003236400086486 & 0.6532357860332504 & 0.7496795347038907 \\
0.8467985984834331 & 0.8470351666391824 & 0.7496162413881409 \\
0.6524933666016351 & 0.4994169239981999 & 0.7492065282408591 \\
0.4999024034580377 & 0.6529814098574068 & 0.7495611198154706 \\
0.3191653402990162 & 0.4863364415442694 & 0.9166969128540735 \\
\hline
\end{tabular}




\begin{tabular}{|c|c|c|}
\hline & 1562 & \\
\hline & 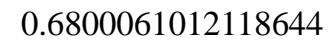 & 0 \\
\hline 0136682094795768 & 8340099253103360 & \\
\hline & 7 & \\
\hline 5 & 55 & \\
\hline & 0. & \\
\hline 9 & & \\
\hline & & \\
\hline & & \\
\hline 66 & 0.83 & 0.0 \\
\hline & & \\
\hline & & \\
\hline-0.0 & 0.4 & 155 \\
\hline 0.50 & & \\
\hline & & \\
\hline 0.8 & & \\
\hline 1572 & 0.662 & 685 \\
\hline 00 & 0.66 & \\
\hline & & \\
\hline 0.1 & 0.8 & 0.3 \\
\hline 0.66 & 0.83 & \\
\hline 0.0 & 0.0 & \\
\hline & & \\
\hline 0.00 & 0.4 & \\
\hline 0.50 & 0.5 & 0.3 \\
\hline 0.33129 & & \\
\hline 0.83 & & \\
\hline 0.33 & 0.66 & \\
\hline 0.83 & & \\
\hline & & \\
\hline 0.66 & 0.3 & \\
\hline 0.16677 & 0.8 & \\
\hline 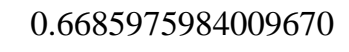 & ט אדים & \\
\hline & & 0.6460 \\
\hline 0.5000 & 0.0000849817858229 & 0.645423277 \\
\hline .0003743378498426 & 0.50040514 & 0.64 \\
\hline & & \\
\hline 0.3330 & 0.166528 & 0.687 \\
\hline 2491 & 0.16 & 0.68 \\
\hline 37 & 0.6 & 0.6 \\
\hline$a^{2}$ & 0.6666269656712 & 0687233 \\
\hline 0.16611319268809 & 0.3330479958585030 & 0.8129563978342 \\
\hline 0.66644210268031 & 0.33 & 0.81 \\
\hline 10021994003510 & & .010 \\
\hline
\end{tabular}




$\begin{array}{ccc}0.6664324406011838 & 0.8332952893133013 & 0.8128480560052086 \\ -0.0005646340972041 & 0.0003056445301202 & 0.8543143826876436 \\ 0.4993126351290447 & -0.0005327080443402 & 0.8541593725155899 \\ -0.0003092232307878 & 0.4991526970527648 & 0.8543808844398877 \\ 0.4989536419054743 & 0.4994380798771836 & 0.8539064915833593 \\ 0.3332967892050405 & 0.1669095419018020 & 0.9803351850119979 \\ 0.8313025355385238 & 0.1649971236660367 & 0.9819691842075861 \\ 0.3313689719369503 & 0.6660181941619870 & 0.9801641805635373 \\ 0.8330702193885985 & 0.6664482215612586 & 0.9786453969351679 \\ 0.1694361993251740 & 0.3356693280671835 & 0.3204059823370376\end{array}$

(2.7) $2 \times 2 \times 1$ supercell with the EP localized on Fe7 (-829.879 eV)

$\begin{array}{ccc}0.1793430297835232 & 0.0159812819431974 & 0.0855864564050615 \\ 0.3327088610657294 & 0.3198867713191930 & 0.0838239717841703 \\ 0.4865945210174973 & 0.1680919536928242 & 0.0845103790376145 \\ 0.6818205716985719 & 0.0147333223349237 & 0.0837479908228453 \\ 0.8371680482473264 & 0.3220213395809597 & 0.0864041933478914 \\ 0.9868832624027958 & 0.1668880289526737 & 0.0836923968425444 \\ 0.1804943072419410 & 0.5135632636645882 & 0.0829131115276746 \\ 0.3351321246519354 & 0.8257125381501267 & 0.0797487523432306 \\ 0.4894336253014528 & 0.6643840928275357 & 0.0801414204068724 \\ 0.6805156687079706 & 0.5137614448936932 & 0.0840033650358914 \\ 0.8324279255733715 & 0.8189426551757933 & 0.0830112683387952 \\ 0.9864141835157267 & 0.6687091299604339 & 0.0830468511305931 \\ 0.1558990623727785 & 0.1701812869429325 & 0.2586654278441906 \\ 0.0171377403697591 & 0.3413834164940590 & 0.2582535053711768 \\ 0.3460922652353098 & 0.0015627665618904 & 0.2503017466519284 \\ 0.6566593090847623 & 0.1548393113982131 & 0.2506644240155771 \\ 0.5016441537670288 & 0.3461606034651065 & 0.2500431998230404 \\ 0.8470388738147654 & -0.0009017645422366 & 0.2493352707188716 \\ 0.1432014989390879 & 0.6499829697607330 & 0.2509928305106094 \\ -0.0001516145677507 & 0.8501377954179002 & 0.2494175308469260 \\ 0.3256350080916700 & 0.4732648879989406 & 0.2603262890456435 \\ 0.6470499609032252 & 0.6500929134636801 & 0.2492444231584677 \\ 0.5026480080736623 & 0.8510712821478539 & 0.2506144881108305 \\ 0.8466456197722720 & 0.4973402412593869 & 0.2494466003493346 \\ 0.0222487988440801 & 0.1900253587863953 & 0.4098058237817300 \\ 0.3082879708901565 & 0.3284145677469170 & 0.4122247064473978 \\ 0.1685973980607066 & 0.4765590966163571 & 0.4102409585030347 \\ 0.5123293279323351 & 0.1796575753883691 & 0.4165600517300366 \\ 0.8200141734703269 & 0.3341308316441986 & 0.4164836678381246 \\ 0.6648318395373977 & 0.4858397575670521 & 0.4165601841945618 \\ 0.0127408022757872 & 0.6790159113140196 & 0.4171607070243585 \\ 0.3190372723596530 & 0.8313788772754900 & 0.4156718856277226\end{array}$




\begin{tabular}{|c|c|c|}
\hline 18 & 385 & 0.4 \\
\hline 0.5135676492475254 & 0.6797177421860767 & 0.4157960745387846 \\
\hline 8200993873884499 & 0.8338675307549834 & 0.4162546314929642 \\
\hline 6682724575260730 & 0.9876542489291110 & 0.4165324382428827 \\
\hline 841645774893389 & 0.3197077950215233 & $0.58168936 \mathrm{~s}$ \\
\hline 1320118 & 0.1655291202031209 & 0.582917780 \\
\hline 02270964 & 8125028 & 0.582 \\
\hline 02 & 396102 & 0.58 \\
\hline 33797 & 0.1667956806322021 & 5338347 \\
\hline 0.8361010122979653 & 0.0159608709417697 & 0.5810365065249312 \\
\hline 157 & 0.8196 & 0.58 \\
\hline 20201962 & 0.6636298040638199 & 0.58 \\
\hline 0.3342329602551977 & 0.5136321177922823 & 799773167 \\
\hline 0.9863553881866199 & 0.8190363913818843 & 0.58324174 \\
\hline 262 & 0.667 & \\
\hline 0.8322 & 3976 & 465 \\
\hline 0.3469025910858640 & 0.3469232230690486 & 0.7496493 \\
\hline 213030 & 0.00005 & 0.74 \\
\hline 0.00 & & \\
\hline 0.8467926550796497 & 0.346831791 & 0.749 \\
\hline 0.6532196630653277 & 0.0001243575453257 & 0.7490878500670002 \\
\hline 0.500627798 & 0.153 & 0.74 \\
\hline 0737 & 0.8470099697 & 0.74 \\
\hline 0.1530433799470362 & 0.5000146084949652 & 0.7492707853527112 \\
\hline 0.0002484149256665 & 0.6531675203547477 & 0.7496306247346617 \\
\hline .84686095 & 0.8474 & 0.7492 \\
\hline .6528988931279990 & 0.4995633671160712 & 0.749653007 \\
\hline 0.4997843646066135 & 0.6531141220874813 & 0.7493762429468711 \\
\hline 0.3192696407519507 & 0.4869315400386632 & 0.9164791426795174 \\
\hline 52 & 0.18070 & 0.9166644 \\
\hline .0131451176515084 & 0.3330761571909521 & 0.9165606800787310 \\
\hline 0.8202921668823903 & 0.4861484540520888 & 0.9172325144950203 \\
\hline 6667641150106035 & 0.1804829106259778 & 0.9176661849564186 \\
\hline .5144367745017885 & 0.3343507269623048 & 0.9171937750757864 \\
\hline 0.3198102891552934 & 0.9886748360222930 & 0.9158404345459854 \\
\hline 0.1667241672738096 & 0.6810817839555479 & 0.9165776043522401 \\
\hline .0135424204493402 & 0.8341365039644283 & 0.9164419234414580 \\
\hline 0.8202191046406174 & 0.9871142388635038 & 0.9164113126325543 \\
\hline 0.6679208457428787 & 0.6806461385291578 & 0.9152112419317439 \\
\hline 0.5140905374705007 & 0.8339521734520186 & 0.9160511590612179 \\
\hline 0.1667949493461420 & 0.3343845285600273 & 0.0205944506695122 \\
\hline 0.6684659742193875 & 0.3332182864965100 & 0.0228367459255172 \\
\hline 0.1680813753104725 & 0.8367387629470326 & 0.0207637196432472 \\
\hline 6659397152848 & 0.8336055975134 & 0.0199406996395 \\
\hline
\end{tabular}




\begin{tabular}{|c|c|c|}
\hline & & \\
\hline $002+24$ & & 0.1 \\
\hline 0069132184854452 & 50002111604 & \\
\hline 0.4978576729771905 & 30 & \\
\hline 490157446460 & 0 & 0 \\
\hline 0.83 & 0 & 0 . \\
\hline & & \\
\hline & & \\
\hline 0.6 & 0.3 & \\
\hline 0.166 & 0.83188861 & \\
\hline & & \\
\hline 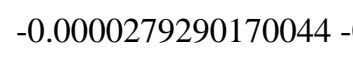 & & 0.35 \\
\hline 0.50 & 307 & 610 \\
\hline 0.00 & 0.5004692099805576 & \\
\hline & & \\
\hline 0.3 & 0. & 0 \\
\hline 0.83 & 0.16889 & 0.47 \\
\hline 0.33 & 0.6 & \\
\hline & & \\
\hline 0.16 & 44048 & 0.52 \\
\hline 0.66 & 0.33 & 0. \\
\hline 10 & 0.8 & \\
\hline 1 & & \\
\hline 0.00 & 0.001 & 0.6461 \\
\hline 0.50 & 0.00 & 0. \\
\hline 4 & 0 . & \\
\hline 0.50 & & \\
\hline 247 & 0.16 & 0.6 \\
\hline 0.83 & 0 & \\
\hline & & 0.68 \\
\hline 24 & 0.6 & 361 \\
\hline 0.1668 & 0.33 & \\
\hline & & \\
\hline & & \\
\hline 2763335738 & 0.8334293 & 0.8124098921736811 \\
\hline .0004332657701792 & 0.00090276 & 0.853 \\
\hline & 0.00 & \\
\hline 0.0008067708277380 & 0.5003987311367 & $0.8542780 €$ \\
\hline 6166048157 & 0.500 & 0.85 \\
\hline 78 & 0.16 & 0.9 \\
\hline 0.8335804247832005 & 0.1666301850406780 & 0.9803588508559977 \\
\hline 0.3336234019315528 & 0.6684992297539692 & 0.9820288944632 \\
\hline 81694790 & 0.666 & 0.9 \\
\hline 100 & 129001 & J.J2 \\
\hline
\end{tabular}


(2.8) $2 \times 2 \times 1$ supercell with the EP localized on Fe8 $(-829.919 \mathrm{eV})$

\begin{tabular}{|c|c|c|}
\hline & & \\
\hline 3337266406651093 & 0.3204396733891730 & 0.0844748708440609 \\
\hline 0.4861758768055737 & 0.1665753141659380 & 0.0838403820549396 \\
\hline 6809296296884763 & 0.0134813496955483 & 0.0841621450045210 \\
\hline 8362891022226434 & 0.3198861248951444 & 0.0850135337845208 \\
\hline 0.9869218614391830 & 0.1657040131711665 & 0.0843532715868267 \\
\hline 0.1803206530847336 & 0.5130065512718365 & 0.0840678909529123 \\
\hline 3337682994042581 & 0.8193943850576622 & 0.083 \\
\hline 4843374625618171 & 0.6642070079642804 & 8643 \\
\hline 0.6772878793982066 & 0.5088819262916081 & 0.0783389359352356 \\
\hline 8309842288886583 & 0.8177624921873576 & 0.0828080086522986 \\
\hline 748180284163 & 0.6673610443018771 & 0.0789059043685146 \\
\hline 759549 & 249895 & 7016 \\
\hline 0.0161601471889467 & 0.3414747075347520 & 0.2589933650220093 \\
\hline .3481090062350332 & 0.0039921518347366 & 0.250 \\
\hline .6557065899274300 & 0.1526745253665213 & 0.25 \\
\hline 715061583004 & 0.3484828864816864 & 0.2491569845678300 \\
\hline 0.8462150236822317 & 0.0013089819197460 & 0.2495021113544139 \\
\hline .1517587576961810 & 0.6536324372743476 & 0.2489186363710547 \\
\hline 015799 & & \\
\hline 0.3227605646904623 & 0.4830287966504351 & 0.2597054816334351 \\
\hline .6359476713552408 & 0.6489468185768176 & 0.2510273045410145 \\
\hline .4963171470343166 & 0.8450526729105241 & 0.2509242551188678 \\
\hline 0.8502890965483061 & 0.4930379582202745 & 0.2492801622573451 \\
\hline 0.0223813668327089 & 0.1911033731766002 & 0.4104250025858397 \\
\hline 0.3087480120668954 & 0.3319767592281586 & 0.4109181104872057 \\
\hline 1672962462524762 & 0.47753706 & 0.410 \\
\hline .5125177329432870 & 0.1806385523596874 & 0.4162351756253340 \\
\hline 0.8196426414931276 & 0.3338212313796540 & 0.4168422979411882 \\
\hline 0.6654717058373883 & 0.4864574643454750 & 0.4156067567200335 \\
\hline .0135129990043725. & 0.6798584881509731 & 01502790568 \\
\hline 0.3194437155321986 & 0.8323355934358325 & 0.4162798011289350 \\
\hline 0.1669060217720507 & 0.9866277302399942 & 0.4174099909662939 \\
\hline o.5132062190375692 & 0.6806761676266143 & 0.4180543391367623 \\
\hline 8201424226098381 & 0.8343078710917672 & 0.4159085218828664 \\
\hline 0.6674815282297709 & 0.9877941683512154 & 0.4165505104524819 \\
\hline 0.4844104105334910 & 0.3203820289706850 & 0.5812704508667882 \\
\hline 0.1796805220856288 & 0.1661980981937190 & 0.5827803805765562 \\
\hline 0.3333312655853428 & 0.0139165887622854 & 0.5825438949653752 \\
\hline 0.9862427940608429 & 0.3202243941947867 & 0.5826372731974971 \\
\hline .6804916264968582 & 0.1668666383800175 & 0.5824577437592402 \\
\hline .8359560208118290 & 0.0157803121004550 & 0.5811928637088328 \\
\hline .486059927786595 & 0.8196217693526668 & 0.5828461192085442 \\
\hline
\end{tabular}




\begin{tabular}{|c|c|c|}
\hline & 0.6642799316547197 & \\
\hline 0.33 & 0.5135725935984461 & 31 \\
\hline 9865172341080704 & 8194208895220028 & \\
\hline 34 & 91 & \\
\hline 2 & 1 & 03 \\
\hline 9 & 4 & 83 \\
\hline & & \\
\hline & & \\
\hline 36 & & \\
\hline ( & & 0.74 \\
\hline & & \\
\hline & & \\
\hline 0.15 & 0.4 & 2248 \\
\hline 0.00 & 0.65 & \\
\hline & & \\
\hline 3 & 0. & 480 \\
\hline 224 & 0.65 & 7290 \\
\hline 85 & 0.4 & \\
\hline & & \\
\hline 25 & 0.3 & 0.9 \\
\hline 941 & $0.4 \varepsilon$ & 0.5 \\
\hline 8 & & \\
\hline & & \\
\hline 52 & 0.98 & \\
\hline 197 & 0.68 & 0.9 \\
\hline 5 & 0.8 & \\
\hline 27 & & \\
\hline 388 & 0.68 & 735 \\
\hline 0.51 & 0.83 & 58 \\
\hline & & \\
\hline 69 & 0.33 & 0.02 \\
\hline 0.16927 & 0.836 & 0.02 \\
\hline & 0.831933512984982 & \\
\hline & & .1448 \\
\hline 0.500011253015 & 0.0000046276859038 & 0.1464764375815939 \\
\hline 38648034878 & 0.5063357382777683 & 479479 \\
\hline & & \\
\hline 3307470334722019 & 0.1706630940179342 & 0.1891962550520143 \\
\hline 093 & 0.16908 & 0.189 \\
\hline & 0.66 & 0.18 \\
\hline 6601 & $0.6617095350090 s$ & 0.1894180014967858 \\
\hline 0.6668479010501692 & 0.3325441053191421 & 0.3121153216703995 \\
\hline 29 & 0.835 & 0.311 \\
\hline 00 & 0.035009150340234 & U. \\
\hline
\end{tabular}




$\begin{array}{clll}0.0014027123158474 & 0.0006753137937559 & 0.3524104653605393 \\ 0.4996063601265303 & 0.0000153660849452 & 0.3537121012023208 \\ -0.0005709126474376 & 0.4993103347996853 & 0.3518967250225940 \\ 0.4982125789447385 & 0.5012191348615880 & 0.3525707623646644 \\ 0.3320773181512707 & 0.1662298177400058 & 0.4784733656295072 \\ 0.8338218875285532 & 0.1683165276401642 & 0.4782946183186941 \\ 0.3334437380394436 & 0.6662312184367498 & 0.4786509632346675 \\ 0.8330800849728275 & 0.6667738310637638 & 0.4780935106962713 \\ 0.1666109050790117 & 0.3334381466443198 & 0.5237350748185693 \\ 0.6660110258803054 & 0.3337409269752804 & 0.5199543758239867 \\ 0.1664302954467327 & 0.8327610537993879 & 0.5198399478981681 \\ 0.6672076857602216 & 0.8339713807670862 & 0.5202798004608655 \\ -0.0001909452453715 & -0.0007689771389965 & 0.6461177375597033 \\ 0.5000266002680036 & 0.0001909174947020 & 0.6455314957932373 \\ -0.0006745324800883 & 0.5001622431384042 & 0.6459995811524653 \\ 0.5006618635788698 & 0.5006442112043900 & 0.6461939365902998 \\ 0.3334767912434308 & 0.1667294151774219 & 0.6871980446472087 \\ 0.8333406005361969 & 0.1664172416118884 & 0.6871295463005185 \\ 0.3333676484907845 & 0.6668075133206444 & 0.6872875735802010 \\ 0.8334291045628978 & 0.6667953918626959 & 0.6867930491072574 \\ 0.1668964799463953 & 0.3332970795776403 & 0.8132258059464098 \\ 0.6670828271013440 & 0.3336329653253120 & 0.8125202832512676 \\ 0.1664926646231378 & 0.8331715460219487 & 0.8128027644313797 \\ 0.6669577865145346 & 0.8330878539644355 & 0.8130923404367932 \\ 0.0003287288201935 & 0.0001605814191586 & 0.8542542137343105 \\ 0.5002098831924213 & -0.0001706852975981 & 0.8539166804012618 \\ 0.0008037308669249 & 0.4998645172585481 & 0.8540365154657537 \\ 0.5001618603545834 & 0.4998975064268694 & 0.8543652327134930 \\ 0.3343625301385121 & 0.1678652306543350 & 0.9801528714961960 \\ 0.8339870693375608 & 0.1655964101942914 & 0.9803381908694977 \\ 0.3327706047801862 & 0.6664594781771475 & 0.9801097947478871 \\ 0.8341501985042896 & 0.6656041610644491 & 0.9806571089038582 \\ 0.1657883966404600 & 0.3333589043484189 & 0.3210143500101547\end{array}$

(2.9) $2 \times 2 \times 1$ supercell with the EP transfer from Fe1 to Fe5 as an example Initial coordinate: $0(-829.858 \mathrm{eV})$
0.1852597094262997
0.0163100845483613
0.0850694260350203
0.3324235780448301
$0.3197457251547390 \quad 0.0842269381227621$
0.4874127121802198
$0.1674006641282402 \quad 0.0844637035981047$
0.6804381314677047
$0.0129713147209025 \quad 0.0843166417247695$
0.8345126560043925
$0.3222669409531168 \quad 0.0858925866834335$
0.9842134267051003
$\begin{array}{ll}0.1742717440001440 & 0.0824769107959521\end{array}$
$\begin{array}{lllll}0.1796393556952068 & 0.5146913700196987 & 0.0839090020926492\end{array}$
$\begin{array}{llll}0.3335623519170481 & 0.8194440412252807 & 0.0844222164454812\end{array}$ 


\begin{tabular}{rll}
0.4830621704847896 & 0.6627739334316222 & 0.0857586609344587 \\
0.6788741452968879 & 0.5090208928575554 & 0.0830729876144729 \\
0.8221001582668690 & 0.8065231084279444 & 0.0820357530872148 \\
0.9870668214175093 & 0.6690029137840428 & 0.0834114314414733 \\
0.1609262305293208 & 0.1780515570189322 & 0.2606859907112975 \\
0.0172803931496926 & 0.3431062874581790 & 0.2589295622242428 \\
0.3470689353999330 & 0.0024447267617426 & 0.2512297207044198 \\
0.6561877735764262 & 0.1530950670743975 & 0.2503867406229499 \\
0.5007622968178487 & 0.3479052769934449 & 0.2496203220828493 \\
0.8465231473835113 & -0.0015239896155017 & 0.2499705064626469 \\
0.1521183261370685 & 0.6526721878486135 & 0.2498585425885110 \\
0.0001473718543373 & 0.8445292946871839 & 0.2541458585068425 \\
0.3264848284295498 & 0.4845351301665262 & 0.2589878380040375 \\
0.6477726909342889 & 0.6501333913881768 & 0.2497191511584183 \\
0.4975598325693182 & 0.8444923776873520 & 0.2509179481394150 \\
0.8497001753467341 & 0.4981824676421842 & 0.2491123329445500 \\
0.0229769852850704 & 0.1912021667818427 & 0.4104499111519438 \\
0.3106115204699557 & 0.3335229685481999 & 0.4115526385300805 \\
0.1686123056436227 & 0.4778058468589493 & 0.4102138249087040 \\
0.5127489303613528 & 0.1809130445051836 & 0.4159534028532043 \\
0.8197227122503857 & 0.3337798406960159 & 0.4160998520593993 \\
0.6662982075002024 & 0.4866577633083360 & 0.4159089237664717 \\
0.0143628529405047 & 0.6780928455171391 & 0.4176831558751842 \\
0.3202867705086996 & 0.8323483922274439 & 0.4165874367539736 \\
0.1663146159325015 & 0.9860454580958760 & 0.4166863011428058 \\
0.5144753871840074 & 0.6803435112186679 & 0.4166094471610683 \\
0.8210187325052316 & 0.8352055986330826 & 0.4163099129971857 \\
0.6678379171466110 & 0.9877155640262324 & 0.4164110521394014 \\
0.4843057362555999 & 0.3201831636418789 & 0.5810728524645407 \\
0.1796383710354916 & 0.1658656799809513 & 0.5825982408057737 \\
0.3335249313013355 & 0.0138929077571194 & 0.5824169314537059 \\
0.9863371479897197 & 0.3205238096252156 & 0.5822433446127732 \\
0.6804645062232941 & 0.1668001130365180 & 0.5821858596420810 \\
0.8361599893337908 & 0.0161252202194075 & 0.5812929458364964 \\
0.4865863140719502 & 0.8197922337701096 & 0.5823418992320442 \\
0.1801601593604236 & 0.6641084119562678 & 0.5813533889796640 \\
0.3340753516381485 & 0.5137915085522401 & 0.5824425355480087 \\
0.9866791644459112 & 0.8194211974354337 & 0.5831929318754069 \\
0.8325004186712093 & 0.5132675650452145 & 0.5830156224180287 \\
\hline
\end{tabular}




\begin{tabular}{|c|c|c|}
\hline & & \\
\hline 44 & 0.1532957151415 & 0.749190 \\
\hline 3466532805426753 & 8470143721539104 & \\
\hline & 9 & 0.749 \\
\hline 29 & 34 & 0 \\
\hline 0.84 & 852 & 0.7 \\
\hline 0 & & \\
\hline & & \\
\hline & & \\
\hline 536026 & 0.1804 & \\
\hline & & \\
\hline & & \\
\hline 0.666 & 0.17 & 147 \\
\hline 0.51 & 0.332 & 0.91 \\
\hline & & \\
\hline 4 & 0. & \\
\hline 41 & 0.83 & \\
\hline 85 & 0.98 & \\
\hline & & \\
\hline 729 & 0.8 & 0.916 \\
\hline 681 & 0.33 & 0.0 \\
\hline 5 & 0. & \\
\hline 90 & & \\
\hline 90 & 0.831 & 0.0 \\
\hline 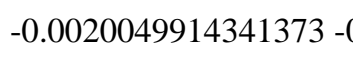 & 0.003 & 0.14 \\
\hline 9 & 0. & \\
\hline 6 & 0.5 & \\
\hline 257 & 0.497 & 0.14 \\
\hline 0.3290 & 0.1 & \\
\hline & & \\
\hline 7 & 0.66 & 0.18 \\
\hline 0.83543 & 0.67 & 28 \\
\hline & & \\
\hline & 0.8332 & 0.313 \\
\hline 0.6666966898258446 & 0.8341171543477491 & 0.3124870697813440 \\
\hline 675765 & 0.00090511 & 124922 \\
\hline 3437 & 0.000 & 0.353 \\
\hline-0.0003456663846943 & 0.4993295264213302 & 0.3523481403858594 \\
\hline 4163 & 0.500 & 0.352 \\
\hline 87 & 0.1 & 0.47 \\
\hline 8343585179054288 & 0.1685093077861091 & 0.4779872815521 \\
\hline 0.3350351605136452 & 0.6664135540715381 & 0.4784283916195 \\
\hline 8 & 0.667 & 0.478 \\
\hline 100 & 351400010392 & 0. \\
\hline
\end{tabular}




$\begin{array}{ccc}0.6659016440231965 & 0.3336717113049547 & 0.5199221531026779 \\ 0.1665452960704009 & 0.8323232318435297 & 0.5205652055403264 \\ 0.6682837613987619 & 0.8345977070237646 & 0.5201626448390084 \\ -0.0002562031504752 & -0.0007557438541711 & 0.6464132840078681 \\ 0.5002196740676007 & 0.0001322283835436 & 0.6456083983514427 \\ -0.0006450950374784 & 0.5002182613243188 & 0.6460147814989758 \\ 0.5008392950272436 & 0.5006156348444358 & 0.6459687475219964 \\ 0.3334473407703586 & 0.1666238346382275 & 0.6871309050945629 \\ 0.8331596386133476 & 0.1666156444199299 & 0.6870612005921588 \\ 0.3332904241981375 & 0.6668271613070692 & 0.6870415703713827 \\ 0.8331737964518510 & 0.6665477804015076 & 0.6871722212084670 \\ 0.1661996997978152 & 0.3334351474792982 & 0.8131082374223362 \\ 0.6666790346455906 & 0.3333341035754428 & 0.8131097356115672 \\ 0.1667947695619371 & 0.8336275637885986 & 0.8131534538762819 \\ 0.6663246095516279 & 0.8326880561651469 & 0.8130817023394734 \\ -0.0000138002985834 & 0.0003051233616631 & 0.8547469773156974 \\ 0.5003804878043511 & 0.0000308672801681 & 0.8541879511380644 \\ 0.0000698952226920 & 0.5004675326937548 & 0.8539227211916349 \\ 0.4984486293474411 & 0.4986376677811525 & 0.8537989721317238 \\ 0.3336192221166527 & 0.1669933269960238 & 0.9805582029094255 \\ 0.8346024125185235 & 0.1663515414030890 & 0.9802197888600864 \\ 0.3323698045148429 & 0.6665263004212161 & 0.9800257412582107 \\ 0.8313658329457239 & 0.6662378518085499 & 0.9788651543396157 \\ 0.1675931087137136 & 0.3345558339641834 & 0.3211092952610840\end{array}$

Interpolating coordinate: $0.167(-829.841 \mathrm{eV})$

$\begin{array}{lrl}0.183548070 & 0.015351816 & 0.084234610 \\ 0.332554649 & 0.319717373 & 0.084001810 \\ 0.487938238 & 0.166963289 & 0.083666156 \\ 0.680627877 & 0.013043451 & 0.084344414 \\ 0.834877739 & 0.321988935 & 0.085838366 \\ 0.984726352 & 0.173105185 & 0.082705758 \\ 0.179692677 & 0.514411467 & 0.083876877 \\ 0.333463108 & 0.819219864 & 0.084299717 \\ 0.483359931 & 0.662773994 & 0.085857110 \\ 0.679455290 & 0.509775357 & 0.083065464 \\ 0.823972983 & 0.808678264 & 0.082362901 \\ 0.986987634 & 0.668759828 & 0.083342182 \\ 0.158719606 & 0.177449039 & 0.260646182 \\ 0.016757334 & 0.343262053 & 0.258895353 \\ 0.347286588 & 0.001595139 & 0.251120253 \\ 0.656057383 & 0.153237549 & 0.250370192 \\ 0.501744823 & 0.349391682 & 0.249852156 \\ 0.846183744 & -0.001538726 & 0.249987945\end{array}$




$\begin{array}{ccc}0.152106110 & 0.652726442 & 0.249774916 \\ 0.000618037 & 0.845925950 & 0.253323953 \\ 0.326086243 & 0.484268586 & 0.258885083 \\ 0.648256141 & 0.650675366 & 0.249665234 \\ 0.497664342 & 0.844311706 & 0.250871929 \\ 0.849785118 & 0.498508414 & 0.249154569 \\ 0.022488875 & 0.191295758 & 0.410750211 \\ 0.310147246 & 0.333173213 & 0.411347415 \\ 0.168446488 & 0.477795974 & 0.410163000 \\ 0.512663091 & 0.180932093 & 0.415896332 \\ 0.819692896 & 0.333957685 & 0.416194247 \\ 0.666266862 & 0.486762400 & 0.416118056 \\ 0.014255163 & 0.678402854 & 0.417441466 \\ 0.320122765 & 0.832246936 & 0.416573585 \\ 0.166284426 & 0.986123835 & 0.416533733 \\ 0.514393812 & 0.680296752 & 0.416589715 \\ 0.821003909 & 0.835220579 & 0.416355836 \\ 0.667743824 & 0.987716476 & 0.416438563 \\ 0.484234230 & 0.320204033 & 0.581063404 \\ 0.179585095 & 0.165861784 & 0.582566787 \\ 0.333500400 & 0.013895683 & 0.582362549 \\ 0.986287848 & 0.320533549 & 0.582367061 \\ 0.680393920 & 0.166860451 & 0.582247570 \\ 0.836040820 & 0.016082235 & 0.581370437 \\ 0.486519070 & 0.819734272 & 0.582355381 \\ 0.180106520 & 0.664092870 & 0.581302639 \\ 0.334032736 & 0.513744886 & 0.582430972 \\ 0.986733908 & 0.819471440 & 0.583171196 \\ 0.680808274 & 0.667469864 & 0.582952948 \\ 0.832532829 & 0.513341261 & 0.583047478 \\ 0.346412620 & 0.346706079 & 0.749327355 \\ 0.153311726 & 0.000209615 & 0.749735227 \\ -0.000072524 & 0.153143992 & 0.749587094 \\ 0.846734377 & 0.346849228 & 0.749403451 \\ 0.653116443 & -0.000414638 & 0.749374893 \\ 0.500359848 & 0.153281115 & 0.749180416 \\ 0.346719005 & 0.846986725 & 0.749203721 \\ 0.153096000 & 0.499957366 & 0.749258334 \\ 0.000469447 & 0.653453173 & 0.749415497 \\ 0.846767784 & 0.847061272 & 0.749592584 \\ 0.652671552 & 0.499380253 & 0.749291520 \\ 0.499791833 & 0.653053892 & 0.749349614 \\ 0.319482532 & 0.486795354 & 0.916630286 \\ 0.166638741 & 0.180458277 & 0.917061728\end{array}$




$\begin{array}{ccc}0.013570898 & 0.333834876 & 0.915950080 \\ 0.819912219 & 0.486062618 & 0.916423456 \\ 0.667140443 & 0.179859817 & 0.916782541 \\ 0.513295249 & 0.332816293 & 0.916410238 \\ 0.320173049 & 0.986596003 & 0.916606987 \\ 0.166725962 & 0.680502984 & 0.916625916 \\ 0.013558764 & 0.833554792 & 0.916720950 \\ 0.819313992 & 0.985327712 & 0.917044084 \\ 0.664839819 & 0.678960872 & 0.915747412 \\ 0.512803961 & 0.832791728 & 0.917051731 \\ 0.165641412 & 0.333455981 & 0.021258402 \\ 0.667614668 & 0.331309018 & 0.020991697 \\ 0.168452508 & 0.835915561 & 0.021249844 \\ 0.664289578 & 0.831320088 & 0.021913636 \\ -0.001197225 & -0.002504439 & 0.143610213 \\ 0.500692902 & 0.000985348 & 0.146300402 \\ 0.000858705 & 0.504425520 & 0.144581151 \\ 0.502613447 & 0.496505811 & 0.144367986 \\ 0.328496399 & 0.167303411 & 0.189531560 \\ 0.840412344 & 0.168139243 & 0.188359271 \\ 0.330905107 & 0.662023036 & 0.189012270 \\ 0.835094739 & 0.671968157 & 0.187096679 \\ 0.666916210 & 0.333029885 & 0.311350435 \\ 0.167836201 & 0.833482562 & 0.313216542 \\ 0.666557311 & 0.833985133 & 0.312529855 \\ 0.001349501 & 0.001308512 & 0.350338470 \\ 0.500279415 & 0.000440480 & 0.353363759 \\ -0.000460877 & 0.499464324 & 0.352413694 \\ 0.499951144 & 0.500168418 & 0.352614688 \\ 0.331611575 & 0.165572679 & 0.478049211 \\ 0.834035579 & 0.168552745 & 0.478111537 \\ 0.334988234 & 0.666353053 & 0.478379291 \\ 0.833760684 & 0.667396633 & 0.478727177 \\ 0.166613212 & 0.333106712 & 0.523699091 \\ 0.665867700 & 0.333775680 & 0.519920633 \\ 0.166582055 & 0.832344699 & 0.520462680 \\ 0.668205766 & 0.834596481 & 0.520155324 \\ -0.000281043 & -0.000759691 & 0.646379574 \\ 0.500168879 & 0.000102703 & 0.645577563 \\ -0.000637724 & 0.500256112 & 0.646033451 \\ 0.500827723 & 0.500642380 & 0.645990091 \\ 0.333463347 & 0.166642441 & 0.687066088 \\ 0.833164286 & 0.166601489 & 0.687107535 \\ 0.333320510 & 0.666775003 & 0.687036516\end{array}$




$\begin{array}{lcl}0.833206995 & 0.666579802 & 0.687180715 \\ 0.166325088 & 0.333373476 & 0.813082932 \\ 0.666683335 & 0.333301295 & 0.813066088 \\ 0.166827733 & 0.833595383 & 0.813021209 \\ 0.666411448 & 0.832750971 & 0.813150144 \\ 0.000129716 & 0.000343923 & 0.854685967 \\ 0.500405904 & -0.00000052 & 0.854183170 \\ 0.000151955 & 0.500308883 & 0.853920014 \\ 0.498656286 & 0.498719711 & 0.853884863 \\ 0.333849834 & 0.166878679 & 0.980793342 \\ 0.834499024 & 0.166184799 & 0.980210519 \\ 0.332489989 & 0.666503105 & 0.980077307 \\ 0.831730215 & 0.666301892 & 0.978828551 \\ 0.167049380 & 0.334424380 & 0.320992054\end{array}$

Interpolating coordinate: $0.333(-829.791 \mathrm{eV})$

$\begin{array}{lcl}0.181836431 & 0.014393548 & 0.083399793 \\ 0.332685720 & 0.319689022 & 0.083776681 \\ 0.488463764 & 0.166525914 & 0.082868609 \\ 0.680817622 & 0.013115588 & 0.084372186 \\ 0.835242823 & 0.321710929 & 0.085784145 \\ 0.985239277 & 0.171938626 & 0.082934605 \\ 0.179745998 & 0.514131564 & 0.083844752 \\ 0.333363864 & 0.818995688 & 0.084177217 \\ 0.483657692 & 0.662774055 & 0.085955559 \\ 0.680036435 & 0.510529820 & 0.083057939 \\ 0.825845809 & 0.810833420 & 0.082690048 \\ 0.986908446 & 0.668516741 & 0.083272932 \\ 0.156512981 & 0.176846520 & 0.260606372 \\ 0.016234276 & 0.343417819 & 0.258861143 \\ 0.347504241 & 0.000745551 & 0.251010784 \\ 0.655926993 & 0.153380030 & 0.250353643 \\ 0.502727349 & 0.350878087 & 0.250083990 \\ 0.845844340 & -0.001553463 & 0.250005384 \\ 0.152093894 & 0.652780695 & 0.249691290 \\ 0.001088702 & 0.847322605 & 0.252502048 \\ 0.325687658 & 0.484002041 & 0.258782329 \\ 0.648739591 & 0.651217342 & 0.249611316 \\ 0.497768852 & 0.844131035 & 0.250825910 \\ 0.849870060 & 0.498834360 & 0.249196806 \\ 0.022000765 & 0.191389350 & 0.411050511 \\ 0.309682971 & 0.332823457 & 0.411142192 \\ 0.168280671 & 0.477786101 & 0.410112175 \\ 0.512577251 & 0.180951142 & 0.415839260\end{array}$




$\begin{array}{ccc}0.819663081 & 0.334135530 & 0.416288642 \\ 0.666235517 & 0.486867037 & 0.416327188 \\ 0.014147473 & 0.678712863 & 0.417199776 \\ 0.319958759 & 0.832145481 & 0.416559734 \\ 0.166254236 & 0.986202213 & 0.416381164 \\ 0.514312237 & 0.680249992 & 0.416569983 \\ 0.820989085 & 0.835235560 & 0.416401759 \\ 0.667649732 & 0.987717388 & 0.416466074 \\ 0.484162724 & 0.320224902 & 0.581053956 \\ 0.179531819 & 0.165857887 & 0.582535333 \\ 0.333475869 & 0.013898459 & 0.582308166 \\ 0.986238548 & 0.320543288 & 0.582490777 \\ 0.680323334 & 0.166920788 & 0.582309279 \\ 0.835921651 & 0.016039250 & 0.581447928 \\ 0.486451825 & 0.819676310 & 0.582368862 \\ 0.180052881 & 0.664077327 & 0.581251889 \\ 0.333990121 & 0.513698264 & 0.582419409 \\ 0.986788652 & 0.819521683 & 0.583149460 \\ 0.680798063 & 0.667530337 & 0.583024608 \\ 0.832565240 & 0.513414957 & 0.583079334 \\ 0.346520972 & 0.346743386 & 0.749325907 \\ 0.153323329 & 0.000196489 & 0.749655003 \\ -0.000044828 & 0.153134622 & 0.749606223 \\ 0.846791229 & 0.346863563 & 0.749434987 \\ 0.653097976 & -0.000463967 & 0.749402689 \\ 0.500353564 & 0.153266515 & 0.749170668 \\ 0.346784729 & 0.846959078 & 0.749180721 \\ 0.153151823 & 0.499909307 & 0.749295872 \\ 0.000492181 & 0.653377832 & 0.749373722 \\ 0.846767054 & 0.847066587 & 0.749609992 \\ 0.652730177 & 0.499456847 & 0.749356464 \\ 0.499847343 & 0.653078642 & 0.749450799 \\ 0.319586712 & 0.486796769 & 0.916618143 \\ 0.166860935 & 0.180498763 & 0.916947939 \\ 0.013589449 & 0.333633683 & 0.916099447 \\ 0.820062382 & 0.486131146 & 0.916420655 \\ 0.667516038 & 0.179922774 & 0.916583468 \\ 0.513519678 & 0.332886286 & 0.916428007 \\ 0.320115288 & 0.986447536 & 0.916482681 \\ 0.166787882 & 0.680350864 & 0.916566255 \\ 0.013420119 & 0.833242659 & 0.916408091 \\ 0.819450003 & 0.985361119 & 0.917072801 \\ 0.665069951 & 0.679100370 & 0.916041345 \\ 0.513004076 & 0.832853492 & 0.917163699\end{array}$




$\begin{array}{ccc}0.166045585 & 0.333391607 & 0.021130090 \\ 0.667830199 & 0.331410111 & 0.020940568 \\ 0.168312603 & 0.835541539 & 0.020983524 \\ 0.664405433 & 0.831360145 & 0.022099783 \\ -0.000389459 & -0.001571205 & 0.143937692 \\ 0.500374570 & 0.001004501 & 0.146149407 \\ 0.001148124 & 0.504354166 & 0.144628924 \\ 0.502338169 & 0.495836010 & 0.144567788 \\ 0.327937624 & 0.166478602 & 0.189651480 \\ 0.839753067 & 0.167983505 & 0.188546083 \\ 0.331091689 & 0.662047835 & 0.189044849 \\ 0.834756639 & 0.671338884 & 0.187165903 \\ 0.666660067 & 0.333208773 & 0.311397469 \\ 0.167892065 & 0.833669097 & 0.312992123 \\ 0.666417932 & 0.833853112 & 0.312572640 \\ 0.001156920 & 0.001711912 & 0.350782541 \\ 0.500127540 & 0.000330080 & 0.353269724 \\ -0.000576087 & 0.499599121 & 0.352479248 \\ 0.499817256 & 0.499935587 & 0.352578459 \\ 0.331566362 & 0.165626124 & 0.477936665 \\ 0.833712641 & 0.168596182 & 0.478235792 \\ 0.334941308 & 0.666292551 & 0.478330191 \\ 0.833735294 & 0.667465815 & 0.478804478 \\ 0.166447601 & 0.333065374 & 0.523688380 \\ 0.665833757 & 0.333879648 & 0.519919112 \\ 0.166618813 & 0.832366166 & 0.520360154 \\ 0.668127771 & 0.834595255 & 0.520148002 \\ -0.000305882 & -0.000763638 & 0.646345865 \\ 0.500118083 & 0.000073177 & 0.645546727 \\ -0.000630353 & 0.500293964 & 0.646052120 \\ 0.500816152 & 0.500669126 & 0.646011435 \\ 0.333479354 & 0.166661048 & 0.687001271 \\ 0.833168932 & 0.166587333 & 0.687153869 \\ 0.333350595 & 0.666722845 & 0.687031462 \\ 0.833240193 & 0.666611824 & 0.687189208 \\ 0.166450477 & 0.333311804 & 0.813057626 \\ 0.666687635 & 0.333268486 & 0.813022440 \\ 0.166860697 & 0.833563203 & 0.812888964 \\ 0.666498287 & 0.832813886 & 0.813218586 \\ 0.000273232 & 0.000382724 & 0.854624956 \\ 0.500431320 & -0.000030971 & 0.854178389 \\ 0.000234014 & 0.500150232 & 0.853917306 \\ 0.498863942 & 0.498801754 & 0.853970754 \\ 0.334080447 & 0.166764031 & 0.981028482\end{array}$




$\begin{array}{lll}0.834395636 & 0.166018057 & 0.980201250 \\ 0.332610174 & 0.666479909 & 0.980128874 \\ 0.832094596 & 0.666365933 & 0.978791948 \\ 0.166505651 & 0.334292926 & 0.320874814\end{array}$

Interpolating coordinate: $0.417(-829.708 \mathrm{eV})$

$\begin{array}{lrl}0.180980611 & 0.013914414 & 0.082982385 \\ 0.332751256 & 0.319674846 & 0.083664117 \\ 0.488726527 & 0.166307227 & 0.082469835 \\ 0.680912495 & 0.013151656 & 0.084386072 \\ 0.835425365 & 0.321571926 & 0.085757034 \\ 0.985495740 & 0.171355347 & 0.083049028 \\ 0.179772659 & 0.513991613 & 0.083828689 \\ 0.333314242 & 0.818883600 & 0.084115967 \\ 0.483806572 & 0.662774085 & 0.086004783 \\ 0.680327007 & 0.510907052 & 0.083054177 \\ 0.826782222 & 0.811910998 & 0.082853622 \\ 0.986868852 & 0.668395198 & 0.083238307 \\ 0.155409669 & 0.176545261 & 0.260586467 \\ 0.015972746 & 0.343495702 & 0.258844038 \\ 0.347613067 & 0.000320757 & 0.250956050 \\ 0.655861798 & 0.153451271 & 0.250345368 \\ 0.503218612 & 0.351621289 & 0.250199907 \\ 0.845674638 & -0.001560831 & 0.250014103 \\ 0.152087786 & 0.652807822 & 0.249649477 \\ 0.001324035 & 0.848020932 & 0.252091095 \\ 0.325488365 & 0.483868769 & 0.258730952 \\ 0.648981316 & 0.651488330 & 0.249584358 \\ 0.497821107 & 0.844040700 & 0.250802900 \\ 0.849912532 & 0.498997333 & 0.249217924 \\ 0.021756710 & 0.191436146 & 0.411200661 \\ 0.309450834 & 0.332648579 & 0.411039580 \\ 0.168197763 & 0.477781165 & 0.410086762 \\ 0.512534331 & 0.180960667 & 0.415810725 \\ 0.819648173 & 0.334224452 & 0.416335839 \\ 0.666219845 & 0.486919355 & 0.416431755 \\ 0.014093628 & 0.678867867 & 0.417078931 \\ 0.319876756 & 0.832094753 & 0.416552808 \\ 0.166239141 & 0.986241402 & 0.416304880 \\ 0.514271450 & 0.680226612 & 0.416560117 \\ 0.820981673 & 0.835243050 & 0.416424721 \\ 0.667602686 & 0.987717844 & 0.416479830 \\ 0.484126971 & 0.320235337 & 0.581049232 \\ 0.179505181 & 0.165855939 & 0.582519607\end{array}$




$\begin{array}{rrr}0.333463603 & 0.013899847 & 0.582280974 \\ 0.986213898 & 0.320548157 & 0.582552635 \\ 0.680288041 & 0.166950957 & 0.582340134 \\ 0.835862067 & 0.016017757 & 0.581486673 \\ 0.486418203 & 0.819647329 & 0.582375602 \\ 0.180026061 & 0.664069556 & 0.581226514 \\ 0.333968813 & 0.513674953 & 0.582413628 \\ 0.986816024 & 0.819546804 & 0.583138592 \\ 0.680792957 & 0.667560573 & 0.583060439 \\ 0.832581445 & 0.513451805 & 0.583095262 \\ 0.346575148 & 0.346762039 & 0.749325183 \\ 0.153329131 & 0.000189926 & 0.749614891 \\ -0.000030980 & 0.153129937 & 0.749615788 \\ 0.846819655 & 0.346870730 & 0.749450756 \\ 0.653088742 & -0.000488631 & 0.749416587 \\ 0.500350422 & 0.153259215 & 0.749165794 \\ 0.346817591 & 0.846945254 & 0.749169221 \\ 0.153179735 & 0.499885278 & 0.749314641 \\ 0.000503548 & 0.653340161 & 0.749352835 \\ 0.846766689 & 0.847069245 & 0.749618696 \\ 0.652759490 & 0.499495144 & 0.749388936 \\ 0.499875098 & 0.653091017 & 0.749501391 \\ 0.319638802 & 0.486797477 & 0.916612072 \\ 0.166972032 & 0.180519006 & 0.916891045 \\ 0.013598725 & 0.333533087 & 0.916174130 \\ 0.820137464 & 0.486165409 & 0.916419255 \\ 0.667703835 & 0.179954252 & 0.916483931 \\ 0.513631892 & 0.332921282 & 0.916436892 \\ 0.320086408 & 0.986373302 & 0.916420528 \\ 0.166818841 & 0.680274804 & 0.916536425 \\ 0.013350796 & 0.833086593 & 0.916251662 \\ 0.819518009 & 0.985377822 & 0.917087159 \\ 0.665185017 & 0.679170119 & 0.916188311 \\ 0.513104133 & 0.832884375 & 0.917219683 \\ 0.166247672 & 0.333359419 & 0.021065934 \\ 0.667937964 & 0.331460657 & 0.020915003 \\ 0.168242651 & 0.835354529 & 0.020850364 \\ 0.664463360 & 0.831380173 & 0.022192857 \\ 0.000014425 & -0.001104589 & 0.144101431 \\ 0.500215404 & 0.001014078 & 0.146073910 \\ 0.001292833 & 0.504318490 & 0.144652811 \\ 0.502200530 & 0.495501109 & 0.144667690 \\ 0.327658236 & 0.166066197 & 0.189711440 \\ 0.839423428 & 0.167905636 & 0.188639489\end{array}$




$\begin{array}{ccc}0.331184981 & 0.662060235 & 0.189061139 \\ 0.834587589 & 0.671024248 & 0.187200514 \\ 0.666531995 & 0.333298217 & 0.311420986 \\ 0.167919997 & 0.833762365 & 0.312879913 \\ 0.666348243 & 0.833787101 & 0.312594033 \\ 0.001060629 & 0.001913613 & 0.351004576 \\ 0.500051603 & 0.000274880 & 0.353222707 \\ -0.000633692 & 0.499666519 & 0.352512025 \\ 0.499750312 & 0.499819171 & 0.352560344 \\ 0.331543756 & 0.165652847 & 0.477880392 \\ 0.833551171 & 0.168617900 & 0.478297920 \\ 0.334917845 & 0.666262300 & 0.478305641 \\ 0.833722599 & 0.667500406 & 0.478843128 \\ 0.166364796 & 0.333044705 & 0.523683024 \\ 0.665816785 & 0.333931632 & 0.519918352 \\ 0.166637193 & 0.832376900 & 0.520308891 \\ 0.668088774 & 0.834594642 & 0.520144341 \\ -0.000318302 & -0.000765611 & 0.646329010 \\ 0.500092686 & 0.000058414 & 0.645531309 \\ -0.000626667 & 0.500312889 & 0.646061455 \\ 0.500810366 & 0.500682499 & 0.646022107 \\ 0.333487357 & 0.166670351 & 0.686968862 \\ 0.833171255 & 0.166580255 & 0.687177036 \\ 0.333365638 & 0.666696766 & 0.687028935 \\ 0.833256792 & 0.666627835 & 0.687193455 \\ 0.166513171 & 0.333280968 & 0.813044973 \\ 0.666689786 & 0.333252081 & 0.813000616 \\ 0.166877179 & 0.833547113 & 0.812822842 \\ 0.666541706 & 0.832845344 & 0.813252807 \\ 0.000344990 & 0.000402124 & 0.854594451 \\ 0.500444027 & -0.000046431 & 0.854175998 \\ 0.000275043 & 0.500070907 & 0.853915952 \\ 0.498967770 & 0.498842776 & 0.854013700 \\ 0.334195753 & 0.166706707 & 0.981146052 \\ 0.834343942 & 0.165934686 & 0.980196615 \\ 0.332670266 & 0.666468311 & 0.980154657 \\ 0.832276787 & 0.666397953 & 0.978773647 \\ 0.166233787 & 0.334227199 & 0.320816194 \\ & & \end{array}$

Interpolating coordinate: $0.420(-829.693 \mathrm{eV})$

$\begin{array}{lll}0.180946378 & 0.013895249 & 0.082965689 \\ 0.332753877 & 0.319674279 & 0.083659614 \\ 0.488737037 & 0.166298479 & 0.082453884 \\ 0.680916290 & 0.013153099 & 0.084386627\end{array}$




$\begin{array}{lcl}0.835432667 & 0.321566366 & 0.085755950 \\ 0.985505998 & 0.171332016 & 0.083053605 \\ 0.179773725 & 0.513986015 & 0.083828047 \\ 0.333312257 & 0.818879116 & 0.084113517 \\ 0.483812527 & 0.662774086 & 0.086006752 \\ 0.680338630 & 0.510922141 & 0.083054027 \\ 0.826819678 & 0.811954101 & 0.082860165 \\ 0.986867268 & 0.668390336 & 0.083236922 \\ 0.155365537 & 0.176533211 & 0.260585671 \\ 0.015962285 & 0.343498817 & 0.258843354 \\ 0.347617420 & 0.000303765 & 0.250953861 \\ 0.655859190 & 0.153454121 & 0.250345037 \\ 0.503238263 & 0.351651017 & 0.250204544 \\ 0.845667850 & -0.001561126 & 0.250014452 \\ 0.152087542 & 0.652808907 & 0.249647804 \\ 0.001333448 & 0.848048865 & 0.252074657 \\ 0.325480393 & 0.483863438 & 0.258728897 \\ 0.648990985 & 0.651499169 & 0.249583280 \\ 0.497823197 & 0.844037087 & 0.250801980 \\ 0.849914231 & 0.499003852 & 0.249218769 \\ 0.021746948 & 0.191438018 & 0.411206667 \\ 0.309441549 & 0.332641584 & 0.411035476 \\ 0.168194447 & 0.477780968 & 0.410085746 \\ 0.512532614 & 0.180961048 & 0.415809584 \\ 0.819647577 & 0.334228009 & 0.416337727 \\ 0.666219218 & 0.486921448 & 0.416435938 \\ 0.014091474 & 0.678874067 & 0.417074097 \\ 0.319873476 & 0.832092724 & 0.416552531 \\ 0.166238537 & 0.986242970 & 0.416301829 \\ 0.514269818 & 0.680225677 & 0.416559722 \\ 0.820981377 & 0.835243350 & 0.416425639 \\ 0.667600804 & 0.987717862 & 0.416480380 \\ 0.484125541 & 0.320235754 & 0.581049043 \\ 0.179504115 & 0.165855861 & 0.582518978 \\ 0.333463112 & 0.013899903 & 0.582279886 \\ 0.986212912 & 0.320548352 & 0.582555109 \\ 0.680286629 & 0.166952164 & 0.582341368 \\ 0.835859684 & 0.016016897 & 0.581488223 \\ 0.486416858 & 0.819646170 & 0.582375872 \\ 0.180024988 & 0.664069245 & 0.581225499 \\ 0.333967961 & 0.513674021 & 0.582413397 \\ 0.986817119 & 0.819547809 & 0.583138157 \\ 0.680792753 & 0.667561782 & 0.583061872 \\ 0.832582093 & 0.513453279 & 0.583095899\end{array}$




$\begin{array}{ccc}0.346577315 & 0.346762785 & 0.749325154 \\ 0.153329363 & 0.000189663 & 0.749613287 \\ -0.000030426 & 0.153129750 & 0.749616171 \\ 0.846820792 & 0.346871017 & 0.749451387 \\ 0.653088373 & -0.000489618 & 0.749417143 \\ 0.500350296 & 0.153258923 & 0.749165599 \\ 0.346818905 & 0.846944701 & 0.749168761 \\ 0.153180851 & 0.499884317 & 0.749315392 \\ 0.000504003 & 0.653338654 & 0.749351999 \\ 0.846766674 & 0.847069351 & 0.749619044 \\ 0.652760663 & 0.499496676 & 0.749390235 \\ 0.499876208 & 0.653091512 & 0.749503415 \\ 0.319640886 & 0.486797505 & 0.916611829 \\ 0.166976476 & 0.180519816 & 0.916888769 \\ 0.013599096 & 0.333529063 & 0.916177117 \\ 0.820140467 & 0.486166780 & 0.916419199 \\ 0.667711347 & 0.179955511 & 0.916479950 \\ 0.513636381 & 0.332922682 & 0.916437247 \\ 0.320085253 & 0.986370333 & 0.916418042 \\ 0.166820079 & 0.680271762 & 0.916535232 \\ 0.013348023 & 0.833080350 & 0.916245405 \\ 0.819520729 & 0.985378490 & 0.917087733 \\ 0.665189620 & 0.679172909 & 0.916194190 \\ 0.513108135 & 0.832885610 & 0.917221922 \\ 0.166255755 & 0.333358132 & 0.021063368 \\ 0.667942275 & 0.331462679 & 0.020913980 \\ 0.168239853 & 0.835347049 & 0.020845038 \\ 0.664465677 & 0.831380974 & 0.022196580 \\ 0.000030580 & -0.001085924 & 0.144107981 \\ 0.500209037 & 0.001014461 & 0.146070890 \\ 0.001298621 & 0.504317063 & 0.144653766 \\ 0.502195024 & 0.495487713 & 0.144671686 \\ 0.327647060 & 0.166049701 & 0.189713838 \\ 0.839410242 & 0.167902521 & 0.188643225 \\ 0.331188713 & 0.662060731 & 0.189061791 \\ 0.834580827 & 0.671011663 & 0.187201898 \\ 0.666526872 & 0.333301795 & 0.311421927 \\ 0.167921114 & 0.833766096 & 0.312875425 \\ 0.666345455 & 0.833784461 & 0.312594889 \\ 0.001056777 & 0.001921681 & 0.351013457 \\ 0.500048566 & 0.000272672 & 0.353220826 \\ 0.499747596969 & 0.499669215 & 0.352513336 \\ 0.331542852 & 0.499814514 & 0.352559619 \\ & 0.165653916 & 0.477878141\end{array}$




$\begin{array}{rrr}0.833544712 & 0.168618769 & 0.478300405 \\ 0.334916906 & 0.666261090 & 0.478304659 \\ 0.833722091 & 0.667501790 & 0.478844674 \\ 0.166361484 & 0.333043878 & 0.523682810 \\ 0.665816106 & 0.333933711 & 0.519918322 \\ 0.166637928 & 0.832377329 & 0.520306840 \\ 0.668087214 & 0.834594617 & 0.520144195 \\ -0.000318799 & -0.000765690 & 0.646328336 \\ 0.500091670 & 0.000057823 & 0.645530692 \\ -0.000626520 & 0.500313646 & 0.646061828 \\ 0.500810135 & 0.500683034 & 0.646022534 \\ 0.333487677 & 0.166670723 & 0.686967566 \\ 0.833171348 & 0.166579972 & 0.687177963 \\ 0.333366240 & 0.666695723 & 0.687028834 \\ 0.833257456 & 0.666628475 & 0.687193625 \\ 0.166515679 & 0.333279735 & 0.813044467 \\ 0.666689872 & 0.333251425 & 0.812999743 \\ 0.166877838 & 0.833546469 & 0.812820197 \\ 0.666543443 & 0.832846602 & 0.813254176 \\ 0.000347860 & 0.000402900 & 0.854593231 \\ 0.500444535 & -0.000047049 & 0.854175902 \\ 0.000276684 & 0.500067734 & 0.853915898 \\ 0.498971923 & 0.498844417 & 0.854015418 \\ 0.334200365 & 0.166704414 & 0.981150755 \\ 0.834341874 & 0.165931351 & 0.980196430 \\ 0.332672670 & 0.666467847 & 0.980155688 \\ 0.832284075 & 0.666399234 & 0.978772915 \\ 0.166222912 & 0.334224570 & 0.320813849 \\ & & \end{array}$

Interpolating coordinate: $0.423(-829.696 \mathrm{eV})$

$\begin{array}{lll}0.180912145 & 0.013876083 & 0.082948992 \\ 0.332756499 & 0.319673712 & 0.083655112 \\ 0.488747548 & 0.166289732 & 0.082437933 \\ 0.680920085 & 0.013154542 & 0.084387183 \\ 0.835439968 & 0.321560806 & 0.085754865 \\ 0.985516257 & 0.171308685 & 0.083058182 \\ 0.179774792 & 0.513980417 & 0.083827404 \\ 0.333310272 & 0.818874633 & 0.084111067 \\ 0.483818482 & 0.662774087 & 0.086008721 \\ 0.680350253 & 0.510937230 & 0.083053876 \\ 0.826857135 & 0.811997204 & 0.082866708 \\ 0.986865684 & 0.668385474 & 0.083235537 \\ 0.155321404 & 0.176521160 & 0.260584875 \\ 0.015951824 & 0.343501933 & 0.258842670\end{array}$




$\begin{array}{lrl}0.347621773 & 0.000286773 & 0.250951671 \\ 0.655856582 & 0.153456970 & 0.250344706 \\ 0.503257913 & 0.351680745 & 0.250209180 \\ 0.845661062 & -0.001561421 & 0.250014801 \\ 0.152087297 & 0.652809992 & 0.249646132 \\ 0.001342861 & 0.848076798 & 0.252058219 \\ 0.325472422 & 0.483858107 & 0.258726842 \\ 0.649000654 & 0.651510009 & 0.249582201 \\ 0.497825287 & 0.844033473 & 0.250801059 \\ 0.849915930 & 0.499010371 & 0.249219614 \\ 0.021737186 & 0.191439890 & 0.411212673 \\ 0.309432263 & 0.332634589 & 0.411031371 \\ 0.168191130 & 0.477780770 & 0.410084729 \\ 0.512530897 & 0.180961429 & 0.415808442 \\ 0.819646980 & 0.334231566 & 0.416339615 \\ 0.666218591 & 0.486923541 & 0.416440120 \\ 0.014089320 & 0.678880267 & 0.417069263 \\ 0.319870196 & 0.832090695 & 0.416552254 \\ 0.166237933 & 0.986244537 & 0.416298777 \\ 0.514268187 & 0.680224742 & 0.416559328 \\ 0.820981080 & 0.835243649 & 0.416426558 \\ 0.667598922 & 0.987717880 & 0.416480930 \\ 0.484124111 & 0.320236172 & 0.581048854 \\ 0.179503050 & 0.165855783 & 0.582518349 \\ 0.333462622 & 0.013899958 & 0.582278799 \\ 0.986211926 & 0.320548547 & 0.582557584 \\ 0.680285217 & 0.166953371 & 0.582342602 \\ 0.835857300 & 0.016016037 & 0.581489773 \\ 0.486415513 & 0.819645011 & 0.582376141 \\ 0.180023915 & 0.664068934 & 0.581224484 \\ 0.333967109 & 0.513673088 & 0.582413166 \\ 0.986818214 & 0.819548814 & 0.583137722 \\ 0.680792549 & 0.667562992 & 0.583063305 \\ 0.832582741 & 0.513454753 & 0.583096536 \\ 0.346579482 & 0.346763531 & 0.749325125 \\ 0.153329595 & 0.000189401 & 0.749611682 \\ -0.000029872 & 0.153129562 & 0.749616553 \\ 0.846821929 & 0.346871303 & 0.749452018 \\ 0.653088003 & -0.000490604 & 0.749417699 \\ 0.500350171 & 0.153258631 & 0.749165404 \\ 0.346820220 & 0.846944148 & 0.749168301 \\ 0.153181968 & 0.499883356 & 0.749316143 \\ 0.000504457 & 0.653337147 & 0.749351164 \\ 0.846766660 & 0.847069458 & 0.749619392 \\ & & \end{array}$




\begin{tabular}{ccc}
0.652761835 & 0.499498208 & 0.749391534 \\
0.499877318 & 0.653092007 & 0.749505439 \\
0.319642969 & 0.486797533 & 0.916611586 \\
0.166980920 & 0.180520626 & 0.916886493 \\
0.013599467 & 0.333525039 & 0.916180105 \\
0.820143470 & 0.486168150 & 0.916419143 \\
0.667718859 & 0.179956770 & 0.916475968 \\
0.513640869 & 0.332924082 & 0.916437603 \\
0.320084098 & 0.986367363 & 0.916415556 \\
0.166821318 & 0.680268719 & 0.916534039 \\
0.013345250 & 0.833074108 & 0.916239148 \\
0.819523449 & 0.985379158 & 0.917088308 \\
0.665194222 & 0.679175699 & 0.916200068 \\
0.513112137 & 0.832886845 & 0.917224162 \\
0.166263839 & 0.333356844 & 0.021060802 \\
0.667946585 & 0.331464701 & 0.020912958 \\
0.168237055 & 0.835339568 & 0.020839711 \\
0.664467994 & 0.831381775 & 0.022200303 \\
0.000046736 & -0.001067260 & 0.144114530 \\
0.500202671 & 0.001014844 & 0.146067870 \\
0.001304410 & 0.504315636 & 0.144654722 \\
0.502189519 & 0.495474317 & 0.144675682 \\
0.327635885 & 0.166033205 & 0.189716237 \\
0.839397057 & 0.167899406 & 0.188646961 \\
0.331192444 & 0.662061227 & 0.189062442 \\
0.834574065 & 0.670999077 & 0.187203283 \\
0.666521749 & 0.333305373 & 0.311422867 \\
0.167922231 & 0.833769827 & 0.312870936 \\
0.666342668 & 0.833781820 & 0.312595744 \\
0.001052926 & 0.001929749 & 0.351022339 \\
0.500045528 & 0.000270464 & 0.353218946 \\
-0.000638300 & 0.499671911 & 0.352514647 \\
0.499744956 & 0.499809858 & 0.352558895 \\
0.331541948 & 0.165654985 & 0.477875890 \\
0.833538253 & 0.168619638 & 0.478302890 \\
0.334915968 & 0.666259880 & 0.478303677 \\
0.833721583 & 0.667503173 & 0.478846220 \\
0.166358172 & 0.333043051 & 0.523682596 \\
0.665815427 & 0.333935791 & 0.519918291 \\
0.166638663 & 0.832377759 & 0.520304790 \\
0.668085654 & 0.834594593 & 0.520144048 \\
0.000319296 & -0.000765769 & 0.646327662 \\
\hline .000626372 & 0.000057233 & 0.645530075 \\
& 0.500314403 & 0.646062202
\end{tabular}




$\begin{array}{lrl}0.500809903 & 0.500683569 & 0.646022961 \\ 0.333487997 & 0.166671095 & 0.686966269 \\ 0.833171441 & 0.166579689 & 0.687178889 \\ 0.333366842 & 0.666694680 & 0.687028733 \\ 0.833258120 & 0.666629116 & 0.687193795 \\ 0.166518187 & 0.333278501 & 0.813043961 \\ 0.666689958 & 0.333250769 & 0.812998870 \\ 0.166878497 & 0.833545826 & 0.812817552 \\ 0.666545180 & 0.832847861 & 0.813255545 \\ 0.000350731 & 0.000403676 & 0.854592011 \\ 0.500445044 & -0.000047668 & 0.854175807 \\ 0.000278325 & 0.500064561 & 0.853915844 \\ 0.498976076 & 0.498846058 & 0.854017136 \\ 0.334204977 & 0.166702121 & 0.981155458 \\ 0.834339806 & 0.165928016 & 0.980196244 \\ 0.332675073 & 0.666467383 & 0.980156720 \\ 0.832291362 & 0.666400515 & 0.978772183 \\ 0.166212038 & 0.334221941 & 0.320811504\end{array}$

Interpolating coordinate: $0.427(-829.696 \mathrm{eV})$

$\begin{array}{lcl}0.180877913 & 0.013856918 & 0.082932296 \\ 0.332759120 & 0.319673145 & 0.083650609 \\ 0.488758058 & 0.166280984 & 0.082421982 \\ 0.680923879 & 0.013155984 & 0.084387738 \\ 0.835447270 & 0.321555245 & 0.085753781 \\ 0.985526515 & 0.171285353 & 0.083062759 \\ 0.179775858 & 0.513974819 & 0.083826762 \\ 0.333308288 & 0.818870149 & 0.084108617 \\ 0.483824438 & 0.662774089 & 0.086010690 \\ 0.680361875 & 0.510952320 & 0.083053726 \\ 0.826894591 & 0.812040307 & 0.082873251 \\ 0.986864101 & 0.668380613 & 0.083234152 \\ 0.155277272 & 0.176509110 & 0.260584078 \\ 0.015941362 & 0.343505048 & 0.258841985 \\ 0.347626126 & 0.000269782 & 0.250949482 \\ 0.655853975 & 0.153459820 & 0.250344375 \\ 0.503277564 & 0.351710474 & 0.250213817 \\ 0.845654274 & -0.001561715 & 0.250015149 \\ 0.152087053 & 0.652811077 & 0.249644459 \\ 0.001352275 & 0.848104732 & 0.252041781 \\ 0.325464450 & 0.483852777 & 0.258724786 \\ 0.649010323 & 0.651520848 & 0.249581123 \\ 0.497827378 & 0.844029860 & 0.250800139 \\ 0.849917628 & 0.499016890 & 0.249220458\end{array}$




$\begin{array}{ccc}0.021727423 & 0.191441761 & 0.411218679 \\ 0.309422978 & 0.332627593 & 0.411027267 \\ 0.168187814 & 0.477780573 & 0.410083713 \\ 0.512529181 & 0.180961810 & 0.415807301 \\ 0.819646384 & 0.334235122 & 0.416341503 \\ 0.666217964 & 0.486925633 & 0.416444303 \\ 0.014087167 & 0.678886468 & 0.417064430 \\ 0.319866915 & 0.832088665 & 0.416551977 \\ 0.166237330 & 0.986246105 & 0.416295726 \\ 0.514266555 & 0.680223806 & 0.416558933 \\ 0.820980784 & 0.835243949 & 0.416427476 \\ 0.667597041 & 0.987717899 & 0.416481481 \\ 0.484122680 & 0.320236589 & 0.581048665 \\ 0.179501984 & 0.165855705 & 0.582517720 \\ 0.333462131 & 0.013900014 & 0.582277711 \\ 0.986210940 & 0.320548741 & 0.582560058 \\ 0.680283806 & 0.166954577 & 0.582343837 \\ 0.835854917 & 0.016015178 & 0.581491322 \\ 0.486414169 & 0.819643851 & 0.582376411 \\ 0.180022843 & 0.664068624 & 0.581223469 \\ 0.333966256 & 0.513672156 & 0.582412934 \\ 0.986819308 & 0.819549818 & 0.583137288 \\ 0.680792344 & 0.667564201 & 0.583064739 \\ 0.832583390 & 0.513456227 & 0.583097174 \\ 0.346581649 & 0.346764278 & 0.749325096 \\ 0.153329827 & 0.000189138 & 0.749610078 \\ -0.000029318 & 0.153129375 & 0.749616936 \\ 0.846823066 & 0.346871590 & 0.749452648 \\ 0.653087634 & -0.000491591 & 0.749418255 \\ 0.500350045 & 0.153258339 & 0.749165209 \\ 0.346821534 & 0.846943595 & 0.749167841 \\ 0.153183084 & 0.499882394 & 0.749316893 \\ 0.000504912 & 0.653335641 & 0.749350328 \\ 0.846766645 & 0.847069564 & 0.749619741 \\ 0.652763008 & 0.499499739 & 0.749392832 \\ 0.499878429 & 0.653092502 & 0.749507462 \\ 0.319645053 & 0.486797562 & 0.916611344 \\ 0.166985364 & 0.180521435 & 0.916884218 \\ 0.013599838 & 0.333521016 & 0.916183092 \\ 0.820146474 & 0.486169521 & 0.916419087 \\ 0.667726370 & 0.179958030 & 0.916471987 \\ 0.513645358 & 0.332925481 & 0.916437958 \\ 0.320082942 & 0.986364394 & 0.916413070 \\ 0.166822556 & 0.680265677 & 0.916532845\end{array}$




$\begin{array}{rrr}0.013342478 & 0.833067865 & 0.916232890 \\ 0.819526170 & 0.985379827 & 0.917088882 \\ 0.665198825 & 0.679178489 & 0.916205947 \\ 0.513116140 & 0.832888081 & 0.917226401 \\ 0.166271922 & 0.333355557 & 0.021058235 \\ 0.667950896 & 0.331466722 & 0.020911935 \\ 0.168234256 & 0.835332088 & 0.020834385 \\ 0.664470312 & 0.831382577 & 0.022204026 \\ 0.000062891 & -0.001048595 & 0.144121080 \\ 0.500196304 & 0.001015227 & 0.146064851 \\ 0.001310198 & 0.504314209 & 0.144655677 \\ 0.502184013 & 0.495460921 & 0.144679678 \\ 0.327624709 & 0.166016708 & 0.189718635 \\ 0.839383871 & 0.167896292 & 0.188650698 \\ 0.331196176 & 0.662061723 & 0.189063094 \\ 0.834567303 & 0.670986492 & 0.187204667 \\ 0.666516627 & 0.333308950 & 0.311423808 \\ 0.167923349 & 0.833773557 & 0.312866448 \\ 0.666339880 & 0.833779180 & 0.312596600 \\ 0.001049074 & 0.001937817 & 0.351031220 \\ 0.500042491 & 0.000268256 & 0.353217065 \\ -0.000640605 & 0.499674607 & 0.352515958 \\ 0.499742279 & 0.499805201 & 0.352558170 \\ 0.331541043 & 0.165656053 & 0.477873639 \\ 0.833531795 & 0.168620506 & 0.478305375 \\ 0.334915029 & 0.666258670 & 0.478302695 \\ 0.833721076 & 0.667504557 & 0.478847766 \\ 0.166354859 & 0.333042225 & 0.523682381 \\ 0.665814749 & 0.333937870 & 0.519918261 \\ 0.166639399 & 0.832378188 & 0.520302739 \\ 0.668084094 & 0.834594568 & 0.520143902 \\ -0.000319792 & -0.000765848 & 0.646326987 \\ 0.500089638 & 0.000056642 & 0.645529459 \\ -0.000626225 & 0.500315160 & 0.646062575 \\ 0.500809672 & 0.500684104 & 0.646023387 \\ 0.333488318 & 0.166671468 & 0.686964973 \\ 0.833171534 & 0.166579405 & 0.687179816 \\ 0.333367443 & 0.666693636 & 0.687028632 \\ 0.833258784 & 0.666629756 & 0.687193964 \\ 0.166520694 & 0.333277268 & 0.813043454 \\ 0.666690044 & 0.333250112 & 0.812997997 \\ 0.166879157 & 0.833545182 & 0.812814907 \\ 0.666546916 & 0.832849119 & 0.813256913 \\ 0.000353601 & 0.000404452 & 0.854590790\end{array}$




$\begin{array}{lrl}0.500445552 & -0.000048286 & 0.854175711 \\ 0.000279967 & 0.500061388 & 0.853915789 \\ 0.498980230 & 0.498847698 & 0.854018853 \\ 0.334209590 & 0.166699828 & 0.981160160 \\ 0.834337739 & 0.165924682 & 0.980196059 \\ 0.332677477 & 0.666466920 & 0.980157751 \\ 0.832298650 & 0.666401795 & 0.978771451 \\ 0.166201163 & 0.334219312 & 0.320809160\end{array}$

Interpolating coordinate: $0.430(-829.697 \mathrm{eV})$

$\begin{array}{lcl}0.180843680 & 0.013837752 & 0.082915599 \\ 0.332761742 & 0.319672578 & 0.083646107 \\ 0.488768569 & 0.166272237 & 0.082406031 \\ 0.680927674 & 0.013157427 & 0.084388294 \\ 0.835454571 & 0.321549685 & 0.085752696 \\ 0.985536774 & 0.171262022 & 0.083067336 \\ 0.179776925 & 0.513969221 & 0.083826119 \\ 0.333306303 & 0.818865666 & 0.084106167 \\ 0.483830393 & 0.662774090 & 0.086012659 \\ 0.680373498 & 0.510967409 & 0.083053575 \\ 0.826932048 & 0.812083410 & 0.082879794 \\ 0.986862517 & 0.668375751 & 0.083232767 \\ 0.155233139 & 0.176497059 & 0.260583282 \\ 0.015930901 & 0.343508164 & 0.258841301 \\ 0.347630479 & 0.000252790 & 0.250947292 \\ 0.655851367 & 0.153462669 & 0.250344044 \\ 0.503297214 & 0.351740202 & 0.250218453 \\ 0.845647486 & -0.001562010 & 0.250015498 \\ 0.152086808 & 0.652812162 & 0.249642787 \\ 0.001361688 & 0.848132665 & 0.252025343 \\ 0.325456479 & 0.483847446 & 0.258722731 \\ 0.649019992 & 0.651531688 & 0.249580044 \\ 0.497829468 & 0.844026246 & 0.250799218 \\ 0.849919327 & 0.499023409 & 0.249221303 \\ 0.021717661 & 0.191443633 & 0.411224685 \\ 0.309413692 & 0.332620598 & 0.411023162 \\ 0.168184497 & 0.477780375 & 0.410082696 \\ 0.512527464 & 0.180962191 & 0.415806159 \\ 0.819645787 & 0.334238679 & 0.416343391 \\ 0.666217337 & 0.486927726 & 0.416448485 \\ 0.014085013 & 0.678892668 & 0.417059596 \\ 0.319863635 & 0.832086636 & 0.416551700 \\ 0.166236726 & 0.986247672 & 0.416292674 \\ 0.514264924 & 0.680222871 & 0.416558539 \\ & & \end{array}$




$\begin{array}{rrr}0.820980487 & 0.835244248 & 0.416428395 \\ 0.667595159 & 0.987717917 & 0.416482031 \\ 0.484121250 & 0.320237007 & 0.581048476 \\ 0.179500919 & 0.165855627 & 0.582517091 \\ 0.333461641 & 0.013900069 & 0.582276624 \\ 0.986209954 & 0.320548936 & 0.582562533 \\ 0.680282394 & 0.166955784 & 0.582345071 \\ 0.835852533 & 0.016014318 & 0.581492872 \\ 0.486412824 & 0.819642692 & 0.582376680 \\ 0.180021770 & 0.664068313 & 0.581222454 \\ 0.333965404 & 0.513671223 & 0.582412703 \\ 0.986820403 & 0.819550823 & 0.583136853 \\ 0.680792140 & 0.667565411 & 0.583066172 \\ 0.832584038 & 0.513457701 & 0.583097811 \\ 0.346583816 & 0.346765024 & 0.749325067 \\ 0.153330059 & 0.000188876 & 0.749608473 \\ -0.000028764 & 0.153129187 & 0.749617318 \\ 0.846824203 & 0.346871876 & 0.749453279 \\ 0.653087264 & -0.000492577 & 0.749418811 \\ 0.500349920 & 0.153258047 & 0.749165014 \\ 0.346822849 & 0.846943042 & 0.749167381 \\ 0.153184201 & 0.499881433 & 0.749317644 \\ 0.000505366 & 0.653334134 & 0.749349493 \\ 0.846766631 & 0.847069671 & 0.749620089 \\ 0.652764180 & 0.499501271 & 0.749394131 \\ 0.499879539 & 0.653092997 & 0.749509486 \\ 0.319647136 & 0.486797590 & 0.916611101 \\ 0.166989808 & 0.180522245 & 0.916881942 \\ 0.013600209 & 0.333516992 & 0.916186080 \\ 0.820149477 & 0.486170891 & 0.916419031 \\ 0.667733882 & 0.179959289 & 0.916468005 \\ 0.513649846 & 0.332926881 & 0.916438314 \\ 0.320081787 & 0.986361424 & 0.916410584 \\ 0.166823795 & 0.680262634 & 0.916531652 \\ 0.013339705 & 0.833061623 & 0.916226633 \\ 0.819528890 & 0.985380495 & 0.917089457 \\ 0.665203427 & 0.679181279 & 0.916211825 \\ 0.513120142 & 0.832889316 & 0.917228641 \\ 0.166280006 & 0.333354269 & 0.021055669 \\ 0.667955206 & 0.331468744 & 0.020910913 \\ 0.168231458 & 0.835324607 & 0.020829058 \\ 0.664472629 & 0.831383378 & 0.022207749 \\ 0.000079047 & -0.001029931 & 0.144127629 \\ 0.500189938 & 0.001015610 & 0.146061831\end{array}$




$\begin{array}{rcc}0.001315987 & 0.504312782 & 0.144656633 \\ 0.502178508 & 0.495447525 & 0.144683674 \\ 0.327613534 & 0.166000212 & 0.189721034 \\ 0.839370686 & 0.167893177 & 0.188654434 \\ 0.331199907 & 0.662062219 & 0.189063745 \\ 0.834560541 & 0.670973906 & 0.187206052 \\ 0.666511504 & 0.333312528 & 0.311424748 \\ 0.167924466 & 0.833777288 & 0.312861959 \\ 0.666337093 & 0.833776539 & 0.312597455 \\ 0.001045223 & 0.001945885 & 0.351040102 \\ 0.500039453 & 0.000266048 & 0.353215185 \\ -0.000642909 & 0.499677303 & 0.352517269 \\ 0.499739601 & 0.499800545 & 0.352557446 \\ 0.331540139 & 0.165657122 & 0.477871388 \\ 0.833525336 & 0.168621375 & 0.478307860 \\ 0.334914091 & 0.666257460 & 0.478301713 \\ 0.833720568 & 0.667505940 & 0.478849312 \\ 0.166351547 & 0.333041398 & 0.523682167 \\ 0.665814070 & 0.333939950 & 0.519918230 \\ 0.166640134 & 0.832378618 & 0.520300689 \\ 0.668082534 & 0.834594544 & 0.520143755 \\ -0.000320289 & -0.000765927 & 0.646326313 \\ 0.500088622 & 0.000056052 & 0.645528842 \\ -0.000626077 & 0.500315917 & 0.646062949 \\ 0.500809440 & 0.500684639 & 0.646023814 \\ 0.333488638 & 0.166671840 & 0.686963676 \\ 0.833171627 & 0.166579122 & 0.687180742 \\ 0.333368045 & 0.666692593 & 0.687028531 \\ 0.833259448 & 0.666630397 & 0.687194134 \\ 0.166523202 & 0.333276034 & 0.813042948 \\ 0.666690130 & 0.333249456 & 0.812997124 \\ 0.166879816 & 0.833544539 & 0.812812262 \\ 0.666548653 & 0.832850378 & 0.813258282 \\ 0.000356472 & 0.000405228 & 0.854589570 \\ 0.500446061 & -0.000048905 & 0.854175616 \\ 0.000281608 & 0.500058215 & 0.853915735 \\ 0.498984383 & 0.498849339 & 0.854020571 \\ 0.334214202 & 0.166697535 & 0.981164863 \\ 0.834335671 & 0.165921347 & 0.980195873 \\ 0.332679880 & 0.666466456 & 0.980158783 \\ 0.832305937 & 0.666403076 & 0.978770719 \\ 0.166190289 & 0.334216683 & 0.320806815\end{array}$

Interpolating coordinate: $0.433(-829.699 \mathrm{eV})$ 


$\begin{array}{lcl}0.180809447 & 0.013818587 & 0.082898903 \\ 0.332764363 & 0.319672011 & 0.083641604 \\ 0.488779079 & 0.166263489 & 0.082390080 \\ 0.680931469 & 0.013158870 & 0.084388849 \\ 0.835461873 & 0.321544125 & 0.085751612 \\ 0.985547032 & 0.171238691 & 0.083071913 \\ 0.179777991 & 0.513963623 & 0.083825477 \\ 0.333304318 & 0.818861182 & 0.084103717 \\ 0.483836348 & 0.662774091 & 0.086014628 \\ 0.680385121 & 0.510982498 & 0.083053425 \\ 0.826969504 & 0.812126513 & 0.082886337 \\ 0.986860933 & 0.668370889 & 0.083231382 \\ 0.155189007 & 0.176485009 & 0.260582486 \\ 0.015920440 & 0.343511279 & 0.258840617 \\ 0.347634832 & 0.000235798 & 0.250945103 \\ 0.655848759 & 0.153465519 & 0.250343713 \\ 0.503316865 & 0.351769930 & 0.250223090 \\ 0.845640698 & -0.001562305 & 0.250015847 \\ 0.152086564 & 0.652813247 & 0.249641114 \\ 0.001371101 & 0.848160598 & 0.252008905 \\ 0.325448507 & 0.483842115 & 0.258720676 \\ 0.649029661 & 0.651542527 & 0.249578966 \\ 0.497831558 & 0.844022633 & 0.250798298 \\ 0.849921026 & 0.499029928 & 0.249222148 \\ 0.021707899 & 0.191445505 & 0.411230691 \\ 0.309404407 & 0.332613603 & 0.411019058 \\ 0.168181181 & 0.477780178 & 0.410081680 \\ 0.512525747 & 0.180962572 & 0.415805018 \\ 0.819645191 & 0.334242236 & 0.416345279 \\ 0.666216710 & 0.486929819 & 0.416452668 \\ 0.014082859 & 0.678898868 & 0.417054762 \\ 0.319860355 & 0.832084607 & 0.416551423 \\ 0.166236122 & 0.986249240 & 0.416289623 \\ 0.514263292 & 0.680221936 & 0.416558144 \\ 0.820980191 & 0.835244548 & 0.416429313 \\ 0.667593277 & 0.987717935 & 0.416482581 \\ 0.484119820 & 0.320237424 & 0.581048287 \\ 0.179499853 & 0.165855549 & 0.582516462 \\ 0.333461150 & 0.013900125 & 0.582275536 \\ 0.986208968 & 0.320549131 & 0.582565007 \\ 0.680280982 & 0.166956991 & 0.582346305 \\ 0.835850150 & 0.016013458 & 0.581494422 \\ 0.486411479 & 0.819641533 & 0.582376950 \\ 0.180020697 & 0.664068002 & 0.581221439\end{array}$




$\begin{array}{rrr}0.333964552 & 0.513670291 & 0.582412472 \\ 0.986821498 & 0.819551828 & 0.583136418 \\ 0.680791936 & 0.667566620 & 0.583067605 \\ 0.832584686 & 0.513459175 & 0.583098448 \\ 0.346585983 & 0.346765770 & 0.749325038 \\ 0.153330291 & 0.000188613 & 0.749606869 \\ -0.000028210 & 0.153129000 & 0.749617701 \\ 0.846825340 & 0.346872163 & 0.749453910 \\ 0.653086895 & -0.000493564 & 0.749419367 \\ 0.500349794 & 0.153257755 & 0.749164819 \\ 0.346824163 & 0.846942489 & 0.749166921 \\ 0.153185317 & 0.499880472 & 0.749318395 \\ 0.000505821 & 0.653332627 & 0.749348657 \\ 0.846766616 & 0.847069777 & 0.749620437 \\ 0.652765353 & 0.499502803 & 0.749395430 \\ 0.499880649 & 0.653093492 & 0.749511510 \\ 0.319649220 & 0.486797618 & 0.916610858 \\ 0.166994252 & 0.180523055 & 0.916879666 \\ 0.013600580 & 0.333512968 & 0.916189067 \\ 0.820152480 & 0.486172262 & 0.916418975 \\ 0.667741394 & 0.179960548 & 0.916464024 \\ 0.513654335 & 0.332928281 & 0.916438669 \\ 0.320080632 & 0.986358455 & 0.916408098 \\ 0.166825033 & 0.680259592 & 0.916530459 \\ 0.013336932 & 0.833055380 & 0.916220376 \\ 0.819531610 & 0.985381163 & 0.917090031 \\ 0.665208030 & 0.679184069 & 0.916217704 \\ 0.513124144 & 0.832890551 & 0.917230880 \\ 0.166288089 & 0.333352982 & 0.021053103 \\ 0.667959517 & 0.331470766 & 0.020909890 \\ 0.168228660 & 0.835317127 & 0.020823732 \\ 0.664474946 & 0.831384179 & 0.022211472 \\ 0.000095202 & -0.001011266 & 0.144134179 \\ 0.500183571 & 0.001015993 & 0.146058811 \\ 0.001321775 & 0.504311355 & 0.144657588 \\ 0.502173002 & 0.495434129 & 0.144687670 \\ 0.327602358 & 0.165983716 & 0.189723432 \\ 0.839357500 & 0.167890062 & 0.188658170 \\ 0.331203639 & 0.662062715 & 0.189064397 \\ 0.834553779 & 0.670961321 & 0.187207436 \\ 0.666506381 & 0.333316106 & 0.311425689 \\ 0.167925583 & 0.833781019 & 0.312857471 \\ 0.666334305 & 0.833773899 & 0.312598311 \\ 0.001041371 & 0.001953953 & 0.351048983\end{array}$




$\begin{array}{rcc}0.500036416 & 0.000263840 & 0.353213304 \\ -0.000645213 & 0.499679999 & 0.352518580 \\ 0.499736923 & 0.499795888 & 0.352556721 \\ 0.331539235 & 0.165658191 & 0.477869137 \\ 0.833518877 & 0.168622244 & 0.478310345 \\ 0.334913152 & 0.666256250 & 0.478300731 \\ 0.833720060 & 0.667507324 & 0.478850858 \\ 0.166348235 & 0.333040571 & 0.523681953 \\ 0.665813391 & 0.333942029 & 0.519918200 \\ 0.166640869 & 0.832379047 & 0.520298638 \\ 0.668080974 & 0.834594519 & 0.520143609 \\ -0.000320786 & -0.000766006 & 0.646325639 \\ 0.500087606 & 0.000055461 & 0.645528225 \\ -0.000625930 & 0.500316674 & 0.646063322 \\ 0.500809209 & 0.500685174 & 0.646024241 \\ 0.333488958 & 0.166672212 & 0.686962380 \\ 0.833171720 & 0.166578839 & 0.687181669 \\ 0.333368647 & 0.666691550 & 0.687028430 \\ 0.833260112 & 0.666631037 & 0.687194304 \\ 0.166525710 & 0.333274801 & 0.813042442 \\ 0.666690216 & 0.333248800 & 0.812996251 \\ 0.166880475 & 0.833543895 & 0.812809617 \\ 0.666550390 & 0.832851636 & 0.813259651 \\ 0.000359342 & 0.000406004 & 0.854588350 \\ 0.500446569 & -0.000049523 & 0.854175520 \\ 0.000283249 & 0.500055042 & 0.853915681 \\ 0.498988536 & 0.498850980 & 0.854022289 \\ 0.334218814 & 0.166695242 & 0.981169566 \\ 0.834333603 & 0.165918012 & 0.980195688 \\ 0.332682284 & 0.666465992 & 0.980159814 \\ 0.832313225 & 0.666404357 & 0.978769987 \\ 0.166179414 & 0.334214054 & 0.320804470 \\ & & \\ & & \end{array}$

Interpolating coordinate: $0.450(-829.709 \mathrm{eV})$

$\begin{array}{lll}0.180638283 & 0.013722760 & 0.082815422 \\ 0.332777470 & 0.319669176 & 0.083619091 \\ 0.488831632 & 0.166219752 & 0.082310325 \\ 0.680950444 & 0.013166083 & 0.084391627 \\ 0.835498381 & 0.321516325 & 0.085746190 \\ 0.985598325 & 0.171122035 & 0.083094798 \\ 0.179783323 & 0.513935632 & 0.083822264 \\ 0.333294393 & 0.818838764 & 0.084091467 \\ 0.483866124 & 0.662774097 & 0.086024473 \\ 0.680443236 & 0.511057945 & 0.083052672\end{array}$




\begin{tabular}{|c|c|c|}
\hline 0.827156787 & 0.812342029 & 0.082919052 \\
\hline 0.986853014 & 0.668346581 & 0.083224457 \\
\hline 0.154968344 & 0.176424757 & 0.260578505 \\
\hline 0.015868134 & 0.343526855 & 0.258837196 \\
\hline 0.347656597 & 0.000150839 & 0.250934156 \\
\hline 0.655835720 & 0.153479767 & 0.250342058 \\
\hline 0.503415118 & 0.351918570 & 0.250246274 \\
\hline 0.845606757 & -0.001563778 & 0.250017591 \\
\hline 0.152085342 & 0.652818673 & 0.249632752 \\
\hline 0.001418168 & 0.848300263 & 0.251926714 \\
\hline 0.325408648 & 0.483815460 & 0.258710401 \\
\hline 0.649078006 & 0.651596725 & 0.249573574 \\
\hline 0.497842009 & 0.844004566 & 0.250793696 \\
\hline 0.849929520 & 0.499062522 & 0.249226371 \\
\hline 0.021659088 & 0.191454864 & 0.411260721 \\
\hline 0.309357979 & 0.332578628 & 0.410998535 \\
\hline 0.168164599 & 0.477779191 & 0.410076597 \\
\hline 0.512517163 & 0.180964477 & 0.415799311 \\
\hline 0.819642210 & 0.334260021 & 0.416354718 \\
\hline 0.666213576 & 0.486940283 & 0.416473581 \\
\hline 0.014072090 & 0.678929869 & 0.417030593 \\
\hline 0.319843955 & 0.832074462 & 0.416550038 \\
\hline 0.166233103 & 0.986257077 & 0.416274366 \\
\hline 0.514255135 & 0.680217260 & 0.416556171 \\
\hline 0.820978708 & 0.835246046 & 0.416433906 \\
\hline 0.667583867 & 0.987718026 & 0.416485332 \\
\hline 0.484112670 & 0.320239511 & 0.581047342 \\
\hline 0.179494526 & 0.165855160 & 0.582513316 \\
\hline 0.333458697 & 0.013900402 & 0.582270098 \\
\hline 0.986204038 & 0.320550105 & 0.582577378 \\
\hline 0.680273923 & 0.166963025 & 0.582352476 \\
\hline 0.835838233 & 0.016009160 & 0.581502171 \\
\hline 0.486404754 & 0.819635737 & 0.582378298 \\
\hline 0.180015333 & 0.664066448 & 0.581216364 \\
\hline 0.333960290 & 0.513665629 & 0.582411315 \\
\hline 0.986826973 & 0.819556853 & 0.583134244 \\
\hline 0.680790915 & 0.667572668 & 0.583074771 \\
\hline 0.832587927 & 0.513466544 & 0.583101633 \\
\hline 0.346596818 & 0.346769501 & 0.749324894 \\
\hline 0.153331452 & 0.000187301 & 0.749598846 \\
\hline-0.000025441 & 0.153128063 & 0.749619614 \\
\hline 0.846831025 & 0.346873597 & 0.749457063 \\
\hline 0.653085048 & -0.000498497 & 0.749422146 \\
\hline 0.500349165 & 0.153256295 & 0.749163845 \\
\hline
\end{tabular}




$\begin{array}{lrl}0.346830736 & 0.846939724 & 0.749164621 \\ 0.153190900 & 0.499875666 & 0.749322149 \\ 0.000508095 & 0.653325093 & 0.749344480 \\ 0.846766543 & 0.847070308 & 0.749622178 \\ 0.652771215 & 0.499510463 & 0.749401925 \\ 0.499886200 & 0.653095967 & 0.749521628 \\ 0.319659638 & 0.486797760 & 0.916609643 \\ 0.167016471 & 0.180527103 & 0.916868287 \\ 0.013602435 & 0.333492848 & 0.916204003 \\ 0.820167496 & 0.486179115 & 0.916418695 \\ 0.667778954 & 0.179966843 & 0.916444117 \\ 0.513676778 & 0.332935281 & 0.916440446 \\ 0.320074856 & 0.986343609 & 0.916395667 \\ 0.166831225 & 0.680244380 & 0.916524493 \\ 0.013323067 & 0.833024167 & 0.916189090 \\ 0.819545211 & 0.985384503 & 0.917092903 \\ 0.665231043 & 0.679198018 & 0.916247097 \\ 0.513144156 & 0.832896728 & 0.917242076 \\ 0.166328507 & 0.333346544 & 0.021040272 \\ 0.667981070 & 0.331480875 & 0.020904777 \\ 0.168214670 & 0.835279725 & 0.020797100 \\ 0.664486531 & 0.831388184 & 0.022230086 \\ 0.000175978 & -0.000917942 & 0.144166927 \\ 0.500151738 & 0.001017908 & 0.146043711 \\ 0.001350717 & 0.504304219 & 0.144662365 \\ 0.502145474 & 0.495367149 & 0.144707650 \\ 0.327546481 & 0.165901235 & 0.189735424 \\ 0.839291572 & 0.167874488 & 0.188676851 \\ 0.331222297 & 0.662065195 & 0.189067655 \\ 0.834519969 & 0.670898394 & 0.187214359 \\ 0.666480767 & 0.333333995 & 0.311430393 \\ 0.167931169 & 0.833799672 & 0.312835029 \\ 0.666320367 & 0.833760697 & 0.312602590 \\ 0.001022113 & 0.001994293 & 0.351093390 \\ 0.500021228 & 0.000252800 & 0.353203900 \\ -0.000656734 & 0.499693479 & 0.352525135 \\ 0.499723534 & 0.499772605 & 0.352553098 \\ 0.331534713 & 0.165663536 & 0.477857883 \\ 0.833486583 & 0.168626587 & 0.478322771 \\ 0.334908460 & 0.666250200 & 0.478295821 \\ 0.833717521 & 0.667514242 & 0.478858588 \\ 0.166331674 & 0.333036437 & 0.523680882 \\ 0.665809996 & 0.333952426 & 0.519918048 \\ 0.166644545 & 0.832381193 & 0.520288386\end{array}$




$\begin{array}{ccc}0.668073175 & 0.834594397 & 0.520142877 \\ -0.000323270 & -0.000766401 & 0.646322268 \\ 0.500082527 & 0.000052509 & 0.645525142 \\ -0.000625193 & 0.500320459 & 0.646065189 \\ 0.500808052 & 0.500687848 & 0.646026375 \\ 0.333490558 & 0.166674072 & 0.686955899 \\ 0.833172185 & 0.166577424 & 0.687186303 \\ 0.333371655 & 0.666686334 & 0.687027924 \\ 0.833263432 & 0.666634239 & 0.687195154 \\ 0.166538249 & 0.333268634 & 0.813039912 \\ 0.666690646 & 0.333245519 & 0.812991886 \\ 0.166883772 & 0.833540677 & 0.812796393 \\ 0.666559074 & 0.832857927 & 0.813266495 \\ 0.000373693 & 0.000409884 & 0.854582249 \\ 0.500449110 & -0.000052615 & 0.854175042 \\ 0.000291455 & 0.500039177 & 0.853915410 \\ 0.499009301 & 0.498859185 & 0.854030878 \\ 0.334241875 & 0.166683778 & 0.981193080 \\ 0.834323264 & 0.165901337 & 0.980194761 \\ 0.332694303 & 0.666463672 & 0.980164970 \\ 0.832349663 & 0.666410761 & 0.978766327 \\ 0.166125041 & 0.334200908 & 0.320792746\end{array}$

Interpolating coordinate: $0.467(-829.718 \mathrm{eV})$

$\begin{array}{lcl}0.180467119 & 0.013626934 & 0.082731940 \\ 0.332790577 & 0.319666340 & 0.083596579 \\ 0.488884184 & 0.166176014 & 0.082230571 \\ 0.680969418 & 0.013173297 & 0.084394404 \\ 0.835534890 & 0.321488524 & 0.085740768 \\ 0.985649617 & 0.171005380 & 0.083117682 \\ 0.179788655 & 0.513907642 & 0.083819052 \\ 0.333284469 & 0.818816347 & 0.084079217 \\ 0.483895901 & 0.662774103 & 0.086034317 \\ 0.680501350 & 0.511133391 & 0.083051920 \\ 0.827344069 & 0.812557544 & 0.082951766 \\ 0.986845096 & 0.668322272 & 0.083217532 \\ 0.154747682 & 0.176364506 & 0.260574525 \\ 0.015815829 & 0.343542432 & 0.258833775 \\ 0.347678363 & 0.000065881 & 0.250923210 \\ 0.655822681 & 0.153494016 & 0.250340404 \\ 0.503513370 & 0.352067211 & 0.250269457 \\ 0.845572817 & -0.001565252 & 0.250019335 \\ 0.152084121 & 0.652824098 & 0.249624389 \\ 0.001465234 & 0.848439929 & 0.251844524\end{array}$




$\begin{array}{lcl}0.325368790 & 0.483788806 & 0.258700125 \\ 0.649126351 & 0.651650922 & 0.249568183 \\ 0.497852460 & 0.843986498 & 0.250789095 \\ 0.849938015 & 0.499095117 & 0.249230595 \\ 0.021610277 & 0.191464224 & 0.411290751 \\ 0.309311552 & 0.332543652 & 0.410978013 \\ 0.168148018 & 0.477778203 & 0.410071515 \\ 0.512508579 & 0.180966381 & 0.415793603 \\ 0.819639228 & 0.334277805 & 0.416364158 \\ 0.666210441 & 0.486950746 & 0.416494495 \\ 0.014061321 & 0.678960869 & 0.417006424 \\ 0.319827554 & 0.832064316 & 0.416548653 \\ 0.166230084 & 0.986264915 & 0.416259110 \\ 0.514246977 & 0.680212584 & 0.416554197 \\ 0.820977226 & 0.835247545 & 0.416438498 \\ 0.667574458 & 0.987718118 & 0.416488083 \\ 0.484105519 & 0.320241597 & 0.581046398 \\ 0.179489198 & 0.165854770 & 0.582510171 \\ 0.333456244 & 0.013900680 & 0.582264659 \\ 0.986199108 & 0.320551079 & 0.582589750 \\ 0.680266865 & 0.166969058 & 0.582358647 \\ 0.835826316 & 0.016004861 & 0.581509920 \\ 0.486398030 & 0.819629940 & 0.582379647 \\ 0.180009969 & 0.664064893 & 0.581211290 \\ 0.333956029 & 0.513660966 & 0.582410159 \\ 0.986832447 & 0.819561877 & 0.583132071 \\ 0.680789893 & 0.667578715 & 0.583081937 \\ 0.832591168 & 0.513473914 & 0.583104819 \\ 0.346607654 & 0.346773231 & 0.749324749 \\ 0.153332612 & 0.000185988 & 0.749590824 \\ -0.000022671 & 0.153127126 & 0.749621526 \\ 0.846836711 & 0.346875030 & 0.749460217 \\ 0.653083202 & -0.000503429 & 0.749424926 \\ 0.500348537 & 0.153254835 & 0.749162870 \\ 0.346837308 & 0.846936960 & 0.749162322 \\ 0.153196482 & 0.499870860 & 0.749325902 \\ 0.000510368 & 0.653317558 & 0.749340302 \\ 0.846766470 & 0.847070840 & 0.749623918 \\ 0.652777078 & 0.499518122 & 0.749408419 \\ 0.499891751 & 0.653098441 & 0.749531747 \\ 0.319670056 & 0.486797901 & 0.916608429 \\ 0.167038691 & 0.180531152 & 0.916856908 \\ 0.013604291 & 0.333472729 & 0.916218940 \\ 0.820182513 & 0.486185967 & 0.916418414\end{array}$




$\begin{array}{ccc}0.667816513 & 0.179973139 & 0.916424209 \\ 0.513699221 & 0.332942280 & 0.91644222 \\ 0.320069079 & 0.986328762 & 0.916383237 \\ 0.166837417 & 0.680229169 & 0.916518526 \\ 0.013309203 & 0.832992953 & 0.916157804 \\ 0.819558813 & 0.985387844 & 0.917095774 \\ 0.665254057 & 0.679211968 & 0.916276491 \\ 0.513164167 & 0.832902904 & 0.917253273 \\ 0.166368924 & 0.333340107 & 0.021027440 \\ 0.668002623 & 0.331490985 & 0.020899664 \\ 0.168200679 & 0.835242322 & 0.020770469 \\ 0.664498117 & 0.831392190 & 0.022248701 \\ 0.000256755 & -0.000824619 & 0.144199675 \\ 0.500119904 & 0.001019824 & 0.146028612 \\ 0.001379659 & 0.504297084 & 0.144667143 \\ 0.502117947 & 0.495300168 & 0.144727631 \\ 0.327490603 & 0.165818754 & 0.189747416 \\ 0.839225645 & 0.167858915 & 0.188695532 \\ 0.331240956 & 0.662067675 & 0.189070912 \\ 0.834486159 & 0.670835466 & 0.187221281 \\ 0.666455152 & 0.333351884 & 0.311435096 \\ 0.167936756 & 0.833818326 & 0.312812588 \\ 0.666306429 & 0.833747494 & 0.312606868 \\ 0.001002854 & 0.002034633 & 0.351137798 \\ 0.500006041 & 0.000241760 & 0.353194497 \\ -0.000668255 & 0.499706958 & 0.352531691 \\ 0.499710146 & 0.499749322 & 0.352549475 \\ 0.331530192 & 0.165668880 & 0.477846628 \\ 0.833454290 & 0.168630931 & 0.478335196 \\ 0.334903767 & 0.666244150 & 0.478290910 \\ 0.833714982 & 0.667521161 & 0.478866319 \\ 0.166315113 & 0.333032303 & 0.523679810 \\ 0.665806602 & 0.333962822 & 0.519917896 \\ 0.166648220 & 0.832383340 & 0.520278133 \\ 0.668065375 & 0.834594274 & 0.520142145 \\ -0.000325754 & -0.000766795 & 0.646318897 \\ 0.500077447 & 0.000049556 & 0.645522058 \\ -0.000624456 & 0.500324245 & 0.646067056 \\ 0.500806894 & 0.500690523 & 0.646028510 \\ 0.333492159 & 0.166675933 & 0.686949417 \\ 0.833172649 & 0.166576008 & 0.687190936 \\ 0.333374664 & 0.666681119 & 0.687027419 \\ 0.833266751 & 0.666637442 & 0.687196003 \\ 0.166550787 & 0.333262467 & 0.813037381\end{array}$




$\begin{array}{lrr}0.666691076 & 0.333242238 & 0.812987522 \\ 0.166887068 & 0.833537458 & 0.812783168 \\ 0.666567758 & 0.832864219 & 0.813273340 \\ 0.000388045 & 0.000413764 & 0.854576148 \\ 0.500451652 & -0.000055706 & 0.854174564 \\ 0.000299661 & 0.500023312 & 0.853915140 \\ 0.499030067 & 0.498867389 & 0.854039467 \\ 0.334264937 & 0.166672313 & 0.981216593 \\ 0.834312925 & 0.165884663 & 0.980193834 \\ 0.332706321 & 0.666461353 & 0.980170127 \\ 0.832386102 & 0.666417166 & 0.978762666 \\ 0.166070669 & 0.334187763 & 0.320781021\end{array}$

Interpolating coordinate: $0.483(-829.727 \mathrm{eV})$

$\begin{array}{lrl}0.180295955 & 0.013531107 & 0.082648459 \\ 0.332803684 & 0.319663505 & 0.083574066 \\ 0.488936737 & 0.166132277 & 0.082150816 \\ 0.680988393 & 0.013180510 & 0.084397182 \\ 0.835571398 & 0.321460724 & 0.085735346 \\ 0.985700910 & 0.170888724 & 0.083140567 \\ 0.179793987 & 0.513879651 & 0.083815839 \\ 0.333274544 & 0.818793929 & 0.084066967 \\ 0.483925677 & 0.662774109 & 0.086044162 \\ 0.680559465 & 0.511208838 & 0.083051167 \\ 0.827531352 & 0.812773060 & 0.082984481 \\ 0.986837177 & 0.668297964 & 0.083210607 \\ 0.154527019 & 0.176304254 & 0.260570544 \\ 0.015763523 & 0.343558008 & 0.258830354 \\ 0.347700128 & -0.000019078 & 0.250912263 \\ 0.655809642 & 0.153508264 & 0.250338749 \\ 0.503611623 & 0.352215851 & 0.250292641 \\ 0.845538876 & -0.001566725 & 0.250021079 \\ 0.152082899 & 0.652829524 & 0.249616027 \\ 0.001512301 & 0.848579594 & 0.251762333 \\ 0.325328931 & 0.483762151 & 0.258689850 \\ 0.649174696 & 0.651705120 & 0.249562791 \\ 0.497862911 & 0.843968431 & 0.250784493 \\ 0.849946509 & 0.499127711 & 0.249234818 \\ 0.021561466 & 0.191473583 & 0.411320781 \\ 0.309265124 & 0.332508677 & 0.410957490 \\ 0.168131436 & 0.477777216 & 0.410066432 \\ 0.512499995 & 0.180968286 & 0.415787896 \\ 0.819636247 & 0.334295590 & 0.416373597 \\ 0.666207307 & 0.486961210 & 0.416515408\end{array}$




$\begin{array}{ccc}0.014050552 & 0.678991870 & 0.416982255 \\ 0.319811154 & 0.832054171 & 0.416547268 \\ 0.166227065 & 0.986272752 & 0.416243853 \\ 0.514238820 & 0.680207908 & 0.41655224 \\ 0.820975743 & 0.835249043 & 0.416443091 \\ 0.667565048 & 0.987718209 & 0.416490834 \\ 0.484098369 & 0.320243684 & 0.581045453 \\ 0.179483871 & 0.165854381 & 0.582507025 \\ 0.333453791 & 0.013900957 & 0.582259221 \\ 0.986194178 & 0.320552053 & 0.582602121 \\ 0.680259806 & 0.166975092 & 0.582364818 \\ 0.835814399 & 0.016000563 & 0.581517669 \\ 0.486391305 & 0.819624144 & 0.582380995 \\ 0.180004605 & 0.664063339 & 0.581206215 \\ 0.333951767 & 0.513656304 & 0.582409002 \\ 0.986837922 & 0.819566902 & 0.583129897 \\ 0.680788872 & 0.667584763 & 0.583089103 \\ 0.832594409 & 0.513481283 & 0.583108004 \\ 0.346618489 & 0.346776962 & 0.749324605 \\ 0.153333773 & 0.000184676 & 0.749582801 \\ -0.000019902 & 0.153126189 & 0.749623439 \\ 0.846842396 & 0.346876464 & 0.749463370 \\ 0.653081355 & -0.000508362 & 0.749427705 \\ 0.500347908 & 0.153253375 & 0.749161896 \\ 0.346843881 & 0.846934195 & 0.749160022 \\ 0.153202065 & 0.499866054 & 0.749329656 \\ 0.000512642 & 0.653310024 & 0.749336125 \\ 0.846766397 & 0.847071371 & 0.749625659 \\ 0.652782940 & 0.499525782 & 0.749414914 \\ 0.499897302 & 0.653100916 & 0.749541865 \\ 0.319680474 & 0.486798043 & 0.916607214 \\ 0.167060910 & 0.180535200 & 0.916845529 \\ 0.013606146 & 0.333452609 & 0.916233876 \\ 0.820197529 & 0.486192820 & 0.916418134 \\ 0.667854073 & 0.179979434 & 0.916404302 \\ 0.513721664 & 0.332949280 & 0.916443999 \\ 0.320063303 & 0.986313916 & 0.916370806 \\ 0.166843609 & 0.680213957 & 0.916512560 \\ 0.013295338 & 0.832961740 & 0.916126518 \\ 0.819572414 & 0.985391184 & 0.917098646 \\ 0.665277070 & 0.679225917 & 0.916305884 \\ 0.513184179 & 0.832909081 & 0.917264469 \\ 0.166409342 & 0.333333669 & 0.021014609 \\ 0.668024176 & 0.331501094 & 0.020894551 \\ & & \end{array}$




$\begin{array}{ccc}0.168186689 & 0.835204920 & 0.020743837 \\ 0.664509702 & 0.831396195 & 0.022267315 \\ 0.000337531 & -0.000731295 & 0.144232423 \\ 0.500088071 & 0.001021739 & 0.146013512 \\ 0.001408601 & 0.504289948 & 0.144671920 \\ 0.502090419 & 0.495233188 & 0.144747611 \\ 0.327434726 & 0.165736273 & 0.189759408 \\ 0.839159717 & 0.167843341 & 0.188714213 \\ 0.331259614 & 0.662070155 & 0.189074170 \\ 0.834452349 & 0.670772539 & 0.187228204 \\ 0.666429538 & 0.333369773 & 0.311439800 \\ 0.167942342 & 0.833836979 & 0.312790146 \\ 0.666292491 & 0.833734292 & 0.312611147 \\ 0.000983596 & 0.002074973 & 0.351182205 \\ 0.499990853 & 0.000230720 & 0.353185093 \\ -0.000679776 & 0.499720438 & 0.352538246 \\ 0.499696757 & 0.499726039 & 0.352545852 \\ 0.331525670 & 0.165674225 & 0.477835374 \\ 0.833421996 & 0.168635274 & 0.478347622 \\ 0.334899075 & 0.666238100 & 0.478286000 \\ 0.833712443 & 0.667528079 & 0.478874049 \\ 0.166298552 & 0.333028169 & 0.523678739 \\ 0.665803207 & 0.333973219 & 0.519917744 \\ 0.166651896 & 0.832385486 & 0.520267881 \\ 0.668057576 & 0.834594152 & 0.520141413 \\ -0.000328238 & -0.000767190 & 0.646315526 \\ 0.500072368 & 0.000046604 & 0.645518975 \\ -0.000623719 & 0.500328030 & 0.646068923 \\ 0.500805737 & 0.500693197 & 0.646030644 \\ 0.333493759 & 0.166677793 & 0.686942936 \\ 0.833173114 & 0.166574593 & 0.687195570 \\ 0.333377672 & 0.666675903 & 0.687026913 \\ 0.833270071 & 0.666640644 & 0.687196853 \\ 0.166563326 & 0.333256300 & 0.813034851 \\ 0.666691506 & 0.333238957 & 0.812983157 \\ 0.166890365 & 0.833534240 & 0.812769944 \\ 0.666576442 & 0.832870510 & 0.813280184 \\ 0.000402396 & 0.000417644 & 0.854570047 \\ 0.500454193 & -0.000058798 & 0.854174086 \\ 0.000307867 & 0.500007447 & 0.853914869 \\ 0.499050832 & 0.498875594 & 0.854048056 \\ 0.334287998 & 0.166660849 & 0.981240107 \\ 0.834302586 & 0.165867988 & 0.980192907 \\ 0.332718340 & 0.666459033 & 0.980175283\end{array}$




$\begin{array}{lll}0.832422540 & 0.666423570 & 0.978759006 \\ 0.166016296 & 0.334174617 & 0.320769297\end{array}$

Interpolating coordinate: 0.500 (-829.736 eV)

$\begin{array}{lrl}0.180124791 & 0.013435280 & 0.082564977 \\ 0.332816791 & 0.319660670 & 0.083551553 \\ 0.488989289 & 0.166088539 & 0.082071061 \\ 0.681007367 & 0.013187724 & 0.084399959 \\ 0.835607906 & 0.321432923 & 0.085729924 \\ 0.985752202 & 0.170772068 & 0.083163452 \\ 0.179799319 & 0.513851661 & 0.083812627 \\ 0.333264620 & 0.818771511 & 0.084054717 \\ 0.483955453 & 0.662774115 & 0.086054007 \\ 0.680617579 & 0.511284284 & 0.083050415 \\ 0.827718634 & 0.812988575 & 0.083017196 \\ 0.986829258 & 0.668273655 & 0.083203682 \\ 0.154306357 & 0.176244002 & 0.260566563 \\ 0.015711217 & 0.343573585 & 0.258826933 \\ 0.347721893 & -0.000104037 & 0.250901316 \\ 0.655796603 & 0.153522512 & 0.250337094 \\ 0.503709876 & 0.352364492 & 0.250315824 \\ 0.845504936 & -0.001568199 & 0.250022823 \\ 0.152081677 & 0.652834949 & 0.249607664 \\ 0.001559367 & 0.848719260 & 0.251680143 \\ 0.325289073 & 0.483735497 & 0.258679574 \\ 0.649223041 & 0.651759317 & 0.249557399 \\ 0.497873362 & 0.843950364 & 0.250779891 \\ 0.849955003 & 0.499160306 & 0.249239042 \\ 0.021512655 & 0.191482942 & 0.411350811 \\ 0.309218697 & 0.332473701 & 0.410936968 \\ 0.168114854 & 0.477776229 & 0.410061350 \\ 0.512491411 & 0.180970191 & 0.415782189 \\ 0.819633265 & 0.334313374 & 0.416383037 \\ 0.666204172 & 0.486971674 & 0.416536321 \\ 0.014039783 & 0.679022871 & 0.416958086 \\ 0.319794753 & 0.832044025 & 0.416545883 \\ 0.166224046 & 0.986280590 & 0.416228596 \\ 0.514230662 & 0.680203232 & 0.416550251 \\ 0.820974261 & 0.835250541 & 0.416447683 \\ 0.667555639 & 0.987718300 & 0.416493585 \\ 0.484091218 & 0.320245771 & 0.581044508 \\ 0.179478543 & 0.165853991 & 0.582503880 \\ 0.333451338 & 0.013901235 & 0.582253783 \\ 0.986189248 & 0.320553027 & 0.582614493\end{array}$




\begin{tabular}{|c|c|c|}
\hline 0.680252747 & 0.166981126 & 0.582370989 \\
\hline 0.835802482 & 0.015996264 & 0.581525418 \\
\hline 0.486384581 & 0.819618348 & 0.582382343 \\
\hline 0.179999241 & 0.664061785 & 0.581201140 \\
\hline 0.333947506 & 0.513651642 & 0.582407846 \\
\hline 0.986843396 & 0.819571926 & 0.583127723 \\
\hline 0.680787851 & 0.667590810 & 0.583096269 \\
\hline 0.832597650 & 0.513488653 & 0.583111190 \\
\hline 0.346629324 & 0.346780693 & 0.749324460 \\
\hline 0.153334933 & 0.000183363 & 0.749574779 \\
\hline-0.000017132 & 0.153125252 & 0.749625352 \\
\hline 0.846848081 & 0.346877897 & 0.749466524 \\
\hline 0.653079508 & -0.000513295 & 0.749430485 \\
\hline 0.500347280 & 0.153251915 & 0.749160921 \\
\hline 0.346850453 & 0.846931430 & 0.749157722 \\
\hline 0.153207647 & 0.499861248 & 0.749333410 \\
\hline 0.000514915 & 0.653302490 & 0.749331947 \\
\hline 0.846766324 & 0.847071903 & 0.749627400 \\
\hline 0.652788803 & 0.499533441 & 0.749421408 \\
\hline 0.499902853 & 0.653103391 & 0.749551984 \\
\hline 0.319690892 & 0.486798184 & 0.916606000 \\
\hline 0.167083130 & 0.180539249 & 0.916834150 \\
\hline 0.013608001 & 0.333432490 & 0.916248813 \\
\hline 0.820212545 & 0.486199673 & 0.916417854 \\
\hline 0.667891632 & 0.179985730 & 0.916384395 \\
\hline 0.513744107 & 0.332956279 & 0.916445776 \\
\hline 0.320057527 & 0.986299069 & 0.916358376 \\
\hline 0.166849801 & 0.680198745 & 0.916506594 \\
\hline 0.013281474 & 0.832930527 & 0.916095232 \\
\hline 0.819586015 & 0.985394525 & 0.917101518 \\
\hline 0.665300083 & 0.679239867 & 0.916335277 \\
\hline 0.513204190 & 0.832915257 & 0.917275666 \\
\hline 0.166449759 & 0.333327232 & 0.021001778 \\
\hline 0.668045729 & 0.331511203 & 0.020889438 \\
\hline 0.168172698 & 0.835167518 & 0.020717205 \\
\hline 0.664521288 & 0.831400201 & 0.022285930 \\
\hline 0.000418308 & -0.000637972 & 0.144265171 \\
\hline 0.500056238 & 0.001023654 & 0.145998413 \\
\hline 0.001437543 & 0.504282813 & 0.144676697 \\
\hline 0.502062891 & 0.495166208 & 0.144767591 \\
\hline 0.327378848 & 0.165653792 & 0.189771400 \\
\hline 0.839093789 & 0.167827767 & 0.188732894 \\
\hline 0.331278272 & 0.662072635 & 0.189077428 \\
\hline 0.834418539 & 0.670709612 & 0.187235126 \\
\hline
\end{tabular}




$\begin{array}{rcc}0.666403924 & 0.333387662 & 0.311444503 \\ 0.167947928 & 0.833855633 & 0.312767704 \\ 0.666278553 & 0.833721090 & 0.312615425 \\ 0.000964338 & 0.002115313 & 0.351226612 \\ 0.499975666 & 0.000219680 & 0.353175690 \\ -0.000691297 & 0.499733918 & 0.352544801 \\ 0.499683368 & 0.499702756 & 0.352542229 \\ 0.331521149 & 0.165679569 & 0.477824119 \\ 0.833389702 & 0.168639618 & 0.478360047 \\ 0.334894382 & 0.666232050 & 0.478281090 \\ 0.833709904 & 0.667534997 & 0.478881779 \\ 0.166281991 & 0.333024035 & 0.523677668 \\ 0.665799813 & 0.333983616 & 0.519917592 \\ 0.166655572 & 0.832387633 & 0.520257628 \\ 0.668049776 & 0.834594029 & 0.520140681 \\ -0.000330722 & -0.000767585 & 0.646312155 \\ 0.500067288 & 0.000043651 & 0.645515891 \\ -0.000622982 & 0.500331815 & 0.646070790 \\ 0.500804580 & 0.500695872 & 0.646032778 \\ 0.333495360 & 0.166679654 & 0.686936454 \\ 0.833173579 & 0.166573177 & 0.687200203 \\ 0.333380681 & 0.666670687 & 0.687026408 \\ 0.833273391 & 0.666643846 & 0.687197702 \\ 0.166575865 & 0.333250133 & 0.813032320 \\ 0.666691936 & 0.333235676 & 0.812978792 \\ 0.166893661 & 0.833531022 & 0.812756719 \\ 0.666585126 & 0.832876802 & 0.813287028 \\ 0.000416748 & 0.000421524 & 0.854563946 \\ 0.500456735 & -0.000061890 & 0.854173608 \\ 0.000316073 & 0.499991582 & 0.853914598 \\ 0.499071598 & 0.498883798 & 0.854056645 \\ 0.334311059 & 0.166649384 & 0.981263621 \\ 0.834292247 & 0.165851314 & 0.980191980 \\ 0.332730358 & 0.666456714 & 0.980180440 \\ 0.832458978 & 0.666429974 & 0.978755346 \\ 0.165961923 & 0.334161472 & 0.320757573 \\ & & \end{array}$

Interpolating coordinate: $0.667(-829.814 \mathrm{eV})$

$\begin{array}{lll}0.178413152 & 0.012477011 & 0.081730160 \\ 0.332947862 & 0.319632318 & 0.083326424 \\ 0.489514815 & 0.165651164 & 0.081273514 \\ 0.681197112 & 0.013259860 & 0.084427731 \\ 0.835972989 & 0.321154917 & 0.085675703 \\ 0.986265127 & 0.169605509 & 0.083392299\end{array}$




$\begin{array}{lrl}0.179852640 & 0.513571758 & 0.083780502 \\ 0.333165376 & 0.818547334 & 0.083932218 \\ 0.484253214 & 0.662774176 & 0.086152456 \\ 0.681198724 & 0.512038748 & 0.083042891 \\ 0.829591459 & 0.815143731 & 0.083344343 \\ 0.986750071 & 0.668030569 & 0.083134432 \\ 0.152099732 & 0.175641483 & 0.260526754 \\ 0.015188158 & 0.343729351 & 0.258792724 \\ 0.347939546 & -0.000953624 & 0.250791848 \\ 0.655666213 & 0.153664994 & 0.250320545 \\ 0.504692402 & 0.353850897 & 0.250547657 \\ 0.845165532 & -0.001582936 & 0.250040262 \\ 0.152069461 & 0.652889203 & 0.249524038 \\ 0.002030032 & 0.850115915 & 0.250858238 \\ 0.324890487 & 0.483468952 & 0.258576819 \\ 0.649706491 & 0.652301292 & 0.249503482 \\ 0.497977872 & 0.843769692 & 0.250733872 \\ 0.850039945 & 0.499486252 & 0.249281278 \\ 0.021024545 & 0.191576533 & 0.411651110 \\ 0.308754422 & 0.332123945 & 0.410731745 \\ 0.167949037 & 0.477766356 & 0.410010525 \\ 0.512405571 & 0.180989240 & 0.415725118 \\ 0.819603449 & 0.334491219 & 0.416477432 \\ 0.666172826 & 0.487076311 & 0.416745453 \\ 0.013932093 & 0.679332880 & 0.416716395 \\ 0.319630747 & 0.831942569 & 0.416532031 \\ 0.166193856 & 0.986358967 & 0.416076027 \\ 0.514149087 & 0.680156473 & 0.416530518 \\ 0.820959437 & 0.835265522 & 0.416493606 \\ 0.667461546 & 0.987719212 & 0.416521096 \\ 0.484019712 & 0.320266640 & 0.581035059 \\ 0.179425267 & 0.165850094 & 0.582472426 \\ 0.333426807 & 0.013904010 & 0.582199400 \\ 0.986139948 & 0.320562766 & 0.582738209 \\ 0.680182161 & 0.167041463 & 0.582432699 \\ 0.835683313 & 0.015953279 & 0.581602909 \\ 0.486317337 & 0.819560386 & 0.582395824 \\ 0.179945602 & 0.664046242 & 0.581150390 \\ 0.333904890 & 0.513605020 & 0.582396283 \\ 0.986898140 & 0.819622169 & 0.583105987 \\ 0.680777640 & 0.667651283 & 0.583167930 \\ 0.832630060 & 0.513562349 & 0.583143046 \\ 0.346737677 & 0.346818000 & 0.749323013 \\ 0.153346537 & 0.000170237 & 0.749494555\end{array}$




$\begin{array}{lrl}0.000010564 & 0.153115882 & 0.749644481 \\ 0.846904933 & 0.346892231 & 0.749498060 \\ 0.653061041 & -0.000562624 & 0.749458282 \\ 0.500340995 & 0.153237316 & 0.749151173 \\ 0.346916177 & 0.846903783 & 0.749134722 \\ 0.153263470 & 0.499813189 & 0.749370948 \\ 0.000537649 & 0.653227149 & 0.749290172 \\ 0.846765594 & 0.847077218 & 0.749644808 \\ 0.652847428 & 0.499610035 & 0.749486352 \\ 0.499958362 & 0.653128141 & 0.749653170 \\ 0.319795071 & 0.486799599 & 0.916593857 \\ 0.167305324 & 0.180579735 & 0.916720361 \\ 0.013626552 & 0.333231296 & 0.916398179 \\ 0.820362709 & 0.486268201 & 0.916415053 \\ 0.668267227 & 0.180048687 & 0.916185322 \\ 0.513968536 & 0.333026272 & 0.916463544 \\ 0.319999765 & 0.986150601 & 0.916234070 \\ 0.166911721 & 0.680046625 & 0.916446933 \\ 0.013142829 & 0.832618394 & 0.915782373 \\ 0.819722026 & 0.985427932 & 0.917130235 \\ 0.665530214 & 0.679379364 & 0.916629210 \\ 0.513404305 & 0.832977021 & 0.917387634 \\ 0.166853933 & 0.333262857 & 0.020873466 \\ 0.668261259 & 0.331612296 & 0.020838309 \\ 0.168032794 & 0.834793497 & 0.020450885 \\ 0.664637143 & 0.831440258 & 0.022472077 \\ 0.001226074 & 0.000295262 & 0.144592650 \\ 0.499737905 & 0.001042806 & 0.145847418 \\ 0.001726963 & 0.504211459 & 0.144724470 \\ 0.501787613 & 0.494496407 & 0.144967393 \\ 0.326820073 & 0.164828983 & 0.189891320 \\ 0.838434512 & 0.167672030 & 0.188919706 \\ 0.331464855 & 0.662097435 & 0.189110007 \\ 0.834080440 & 0.670080339 & 0.187304350 \\ 0.666147782 & 0.333566551 & 0.311491537 \\ 0.168003792 & 0.834042168 & 0.312543284 \\ 0.666139173 & 0.833589069 & 0.312658211 \\ 0.000771756 & 0.002518713 & 0.351670683 \\ 0.499823791 & 0.000109280 & 0.353081655 \\ -0.000806507 & 0.499868716 & 0.352610355 \\ 0.499549481 & 0.499469926 & 0.352506000 \\ 0.331475936 & 0.165733014 & 0.477711573 \\ 0.833066763 & 0.168683055 & 0.478484302 \\ 0.334847456 & 0.666171549 & 0.478231990\end{array}$




$\begin{array}{ccc}0.833684514 & 0.667604180 & 0.478959080 \\ 0.166116381 & 0.332982696 & 0.523666957 \\ 0.665765869 & 0.334087584 & 0.519916071 \\ 0.166692331 & 0.832409100 & 0.520155103 \\ 0.667971781 & 0.834592804 & 0.520133360 \\ -0.000355561 & -0.000771532 & 0.646278445 \\ 0.500016492 & 0.000014125 & 0.645485055 \\ -0.000615611 & 0.500369666 & 0.646089459 \\ 0.500793009 & 0.500722617 & 0.646054122 \\ 0.333511367 & 0.166698261 & 0.686871637 \\ 0.833178226 & 0.166559021 & 0.687246537 \\ 0.333410766 & 0.666618529 & 0.687021354 \\ 0.833306589 & 0.666675867 & 0.687206196 \\ 0.166701254 & 0.333188461 & 0.813007014 \\ 0.666696236 & 0.333202867 & 0.812935144 \\ 0.166926625 & 0.833498841 & 0.812624474 \\ 0.666671964 & 0.832939717 & 0.813355470 \\ 0.000560265 & 0.000460324 & 0.854502935 \\ 0.500482151 & -0.000092809 & 0.854168827 \\ 0.000398133 & 0.499832932 & 0.853911891 \\ 0.499279255 & 0.498965841 & 0.854142536 \\ 0.334541671 & 0.166534736 & 0.981498760 \\ 0.834188859 & 0.165684572 & 0.980182710 \\ 0.332850543 & 0.666433518 & 0.980232006 \\ 0.832823360 & 0.666494015 & 0.978718743 \\ 0.165418194 & 0.334030018 & 0.320640332\end{array}$

Interpolating coordinate: $0.833(-829.863 \mathrm{eV})$

$\begin{array}{lrl}0.176701513 & 0.011518743 & 0.080895344 \\ 0.333078933 & 0.319603966 & 0.083101296 \\ 0.490040341 & 0.165213788 & 0.080475966 \\ 0.681386857 & 0.013331997 & 0.084455503 \\ 0.836338072 & 0.320876911 & 0.085621482 \\ 0.986778053 & 0.168438950 & 0.083621146 \\ 0.179905961 & 0.513291854 & 0.083748377 \\ 0.333066132 & 0.818323157 & 0.083809718 \\ 0.484550975 & 0.662774236 & 0.086250905 \\ 0.681779869 & 0.512793211 & 0.083035367 \\ 0.831464284 & 0.817298887 & 0.083671490 \\ 0.986670883 & 0.667787483 & 0.083065182 \\ 0.149893108 & 0.175038965 & 0.260486945 \\ 0.014665099 & 0.343885117 & 0.258758514 \\ 0.348157198 & -0.001803212 & 0.250682380 \\ 0.655535823 & 0.153807475 & 0.250303996\end{array}$




$\begin{array}{lcl}0.505674929 & 0.355337302 & 0.250779491 \\ 0.844826129 & -0.001597673 & 0.250057701 \\ 0.152057245 & 0.652943457 & 0.249440412 \\ 0.002500697 & 0.851512570 & 0.250036332 \\ 0.324491902 & 0.483202408 & 0.258474065 \\ 0.650189941 & 0.652843267 & 0.249449564 \\ 0.498082382 & 0.843589021 & 0.250687853 \\ 0.850124888 & 0.499812199 & 0.249323515 \\ 0.020536435 & 0.191670125 & 0.411951410 \\ 0.308290147 & 0.331774189 & 0.410526521 \\ 0.167783220 & 0.477756483 & 0.409959699 \\ 0.512319731 & 0.181008288 & 0.415668046 \\ 0.819573633 & 0.334669063 & 0.416571827 \\ 0.666141481 & 0.487180948 & 0.416954585 \\ 0.013824403 & 0.679642888 & 0.416474705 \\ 0.319466741 & 0.831841113 & 0.416518180 \\ 0.166163665 & 0.986437344 & 0.415923459 \\ 0.514067512 & 0.680109713 & 0.416510786 \\ 0.820944613 & 0.835280503 & 0.416539529 \\ 0.667367454 & 0.987720124 & 0.416548607 \\ 0.483948206 & 0.320287510 & 0.581025611 \\ 0.179371991 & 0.165846198 & 0.582440972 \\ 0.333402276 & 0.013906786 & 0.582145017 \\ 0.986090648 & 0.320572505 & 0.582861925 \\ 0.680111575 & 0.167101801 & 0.582494409 \\ 0.835564144 & 0.015910294 & 0.581680400 \\ 0.486250092 & 0.819502424 & 0.582409306 \\ 0.179891963 & 0.664030700 & 0.581099640 \\ 0.333862275 & 0.513558398 & 0.582384719 \\ 0.986952883 & 0.819672411 & 0.583084251 \\ 0.680767428 & 0.667711756 & 0.583239591 \\ 0.832662471 & 0.513636045 & 0.583174902 \\ 0.346846029 & 0.346855307 & 0.749321566 \\ 0.153358140 & 0.000157112 & 0.749414331 \\ 0.000038260 & 0.153106511 & 0.749663610 \\ 0.846961785 & 0.346906566 & 0.749529597 \\ 0.653042573 & -0.000611953 & 0.749486078 \\ 0.500334711 & 0.153222716 & 0.749141425 \\ 0.346981901 & 0.846876136 & 0.749111722 \\ 0.153319294 & 0.499765130 & 0.749408486 \\ 0.000560383 & 0.653151807 & 0.749248397 \\ 0.846764864 & 0.847082533 & 0.749662216 \\ 0.652906054 & 0.499686629 & 0.749551296 \\ 0.500013872 & 0.653152891 & 0.749754355\end{array}$




\begin{tabular}{ccc}
0.319899251 & 0.486801014 & 0.916581714 \\
0.167527519 & 0.180620221 & 0.916606572 \\
0.013645104 & 0.333030103 & 0.916547546 \\
0.820512872 & 0.486336728 & 0.916412252 \\
0.668642822 & 0.180111644 & 0.915986249 \\
0.514192965 & 0.333096265 & 0.916481313 \\
0.319942004 & 0.986002134 & 0.916109764 \\
0.166973641 & 0.679894505 & 0.916387273 \\
0.013004183 & 0.832306261 & 0.915469514 \\
0.819858037 & 0.985461338 & 0.917158952 \\
0.665760346 & 0.679518861 & 0.916923142 \\
0.513604420 & 0.833038786 & 0.917499602 \\
0.167258106 & 0.333198483 & 0.020745155 \\
0.668476789 & 0.331713389 & 0.020787180 \\
0.167892889 & 0.834419476 & 0.020184566 \\
0.664752997 & 0.831480315 & 0.022658224 \\
0.002033841 & 0.001228496 & 0.144920129 \\
0.499419573 & 0.001061959 & 0.145696423 \\
0.002016382 & 0.504140106 & 0.144772244 \\
0.501512335 & 0.493826605 & 0.145167196 \\
0.326261298 & 0.164004174 & 0.190011239 \\
0.837775235 & 0.167516292 & 0.189106518 \\
0.331651438 & 0.662122234 & 0.189142586 \\
0.833742340 & 0.669451067 & 0.187373573 \\
0.665891639 & 0.333745440 & 0.311538571 \\
0.168059656 & 0.834228704 & 0.312318865 \\
0.665999794 & 0.833457048 & 0.312700996 \\
0.000579175 & 0.002922113 & 0.352114755 \\
0.499671916 & -0.000001121 & 0.352987620 \\
-0.000921717 & 0.500003513 & 0.352675909 \\
0.499415593 & 0.499237095 & 0.352469771 \\
0.331430723 & 0.165786458 & 0.477599027 \\
0.832743825 & 0.168726492 & 0.478608557 \\
0.334800529 & 0.666111047 & 0.478182889 \\
0.833659124 & 0.667673362 & 0.479036381 \\
0.165950771 & 0.332941357 & 0.523656245 \\
0.665731926 & 0.334191553 & 0.519914551 \\
0.166729089 & 0.832430567 & 0.520052577 \\
0.667893786 & 0.834591578 & 0.520126039 \\
-0.000380401 & -0.000775479 & 0.646244736 \\
0.499965697 & -0.000015400 & 0.645454219 \\
0.5006088240 & 0.500407517 & 0.646108129 \\
\hline .333527373 & 0.500749363 & 0.646075465 \\
\hline & 0.166716868 & 0.686806819
\end{tabular}




$\begin{array}{lrl}0.833182873 & 0.166544865 & 0.687292872 \\ 0.333440851 & 0.666566371 & 0.687016299 \\ 0.833339787 & 0.666707889 & 0.687214689 \\ 0.166826642 & 0.333126790 & 0.812981708 \\ 0.666700537 & 0.333170058 & 0.812891496 \\ 0.166959589 & 0.833466661 & 0.812492229 \\ 0.666758803 & 0.833002632 & 0.813423912 \\ 0.000703781 & 0.000499124 & 0.854441924 \\ 0.500507567 & -0.000123729 & 0.854164046 \\ 0.000480192 & 0.499674282 & 0.853909183 \\ 0.499486911 & 0.499047884 & 0.854228427 \\ 0.334772284 & 0.166420088 & 0.981733900 \\ 0.834085470 & 0.165517830 & 0.980173441 \\ 0.332970728 & 0.666410323 & 0.980283572 \\ 0.833187741 & 0.666558055 & 0.978682140 \\ 0.164874465 & 0.333898564 & 0.320523091\end{array}$

Final coordinate: 1 (-829.880 eV)

$\begin{array}{lll}0.1749898735305866 & 0.0105604745156309 & 0.0800605276942872 \\ 0.3332100033989742 & 0.3195756142277320 & 0.0828761675266668 \\ 0.4905658668174342 & 0.1647764132582518 & 0.0796784185607199 \\ 0.6815766018853486 & 0.0134041330779668 & 0.0844832756772432 \\ 0.8367031555004855 & 0.3205989047440753 & 0.0855672613467643 \\ 0.9872909778186381 & 0.1672723914719966 & 0.0838499928468934 \\ 0.1799592817457020 & 0.5130119513696290 & 0.0837162523619649 \\ 0.3329668881635272 & 0.8180989804458945 & 0.0836872185133971 \\ 0.4848487355809472 & 0.6627742969720790 & 0.0863493540176857 \\ 0.6823610131805358 & 0.5135476748993186 & 0.0830278430014666 \\ 0.8333371091922026 & 0.8194540425500975 & 0.0839986379272985 \\ 0.9865916951910748 & 0.6675443963855138 & 0.0829959319826519 \\ 0.1476864832006284 & 0.1744364462063885 & 0.2604471357921161 \\ 0.0141420403748356 & 0.3440408832819210 & 0.2587243044660633 \\ 0.3483748509065767 & 0.9973472001437986 & 0.2505729119376723 \\ 0.6554054329784265 & 0.1539499570520180 & 0.2502874475678134 \\ 0.5066574548494640 & 0.3568237069233169 & 0.2510113249397003 \\ 0.8444867249434427 & -0.0016124091909230 & 0.2500751401866345 \\ 0.1520450283203845 & 0.6529977103121594 & 0.2493567858167030 \\ 0.0029713625417543 & 0.8529092254929669 & 0.2492144270123525 \\ 0.3240933166785914 & 0.4829358631419587 & 0.2583713099387243 \\ 0.6506733916083230 & 0.6533852418108618 & 0.2493956471670369 \\ 0.4981868917204742 & 0.8434083494944589 & 0.2506418333846933 \\ 0.8502098303014195 & 0.5001381447221671 & 0.2493657508364977 \\ 0.0200483246691153 & 0.1917637165207022 & 0.4122517101680744 \\ 0.3078258726682321 & 0.3314244333047779 & 0.4103212975511544\end{array}$




\begin{tabular}{llll}
0.1676174025748681 & 0.4777466106395591 & 0.4099088743170074 \\
0.5122338913187904 & 0.1810273370715581 & 0.4156109748953336 \\
0.8195438176104152 & 0.3348469080536384 & 0.4166662224072407 \\
0.6661101359595714 & 0.4872855848245221 & 0.4171637176974179 \\
0.0137167135954361 & 0.6799528965169844 & 0.4162330151314590 \\
0.3193027347752936 & 0.8317396571628692 & 0.4165043285027640 \\
0.1661334753517443 & 0.9865157217145826 & 0.4157708902197823 \\
0.5139859370597955 & 0.6800629537108380 & 0.4164910540041009 \\
0.8209297885598269 & 0.8352954832999312 & 0.4165854522995212 \\
0.6672733609880840 & 0.9877210354059732 & 0.4165761179300499 \\
0.4838767003252981 & 0.3203083788347044 & 0.5810161628531708 \\
0.1793187149639815 & 0.1658423012064220 & 0.5824095182937112 \\
0.3333777450345398 & 0.0139095615845950 & 0.5820906337731873 \\
0.9860413484502283 & 0.3205822440679532 & 0.5829856410862603 \\
0.6800409886410146 & 0.1671621381727293 & 0.5825561190727822 \\
0.8354449745664240 & 0.0158673085871387 & 0.5817578909342702 \\
0.4861828477517010 & 0.8194444625566851 & 0.5824227869869296 \\
0.1798383235047869 & 0.6640151573102939 & 0.5810488904137977 \\
0.3338196594669841 & 0.5135117761962227 & 0.5823731559808378 \\
0.9870076272026900 & 0.8197226540751096 & 0.5830625149127401 \\
0.6807572168112483 & 0.6677722283991480 & 0.5833112517736296 \\
0.8326948814052703 & 0.5137097404324245 & 0.5832067574314400 \\
0.3469543813060654 & 0.3468926140290334 & 0.7493201187194803 \\
0.1533697438512192 & 0.0001439859832305 & 0.7493341074755745 \\
0.0000659559856838 & 0.1530971412080002 & 0.7496827395123471 \\
0.8470186368891590 & 0.3469208998101743 & 0.7495611331788055 \\
0.6530241053076629 & -0.0006612813284676 & 0.7495138741703664 \\
0.5003284268712318 & 0.1532081156856462 & 0.7491316776776301 \\
0.3470476249968955 & 0.8468484884783695 & 0.7490887220960515 \\
0.1533751169759585 & 0.4997170714118014 & 0.7494460239556032 \\
0.0005831169143855 & 0.6530764660752472 & 0.7492066219131897 \\
0.8467641337253680 & 0.8470878485639379 & 0.7496796236978742 \\
0.6529646796173090 & 0.4997632226298636 & 0.7496162401905163 \\
0.5000693819799080 & 0.6531776403119066 & 0.74985555401349168 \\
0.3200034307026711 & 0.4868024294097366 & 0.9165695715455966 \\
0.1677497133071785 & 0.1806607066136907 & 0.9164927831295250 \\
0.0136636552038263 & 0.3328289095971889 & 0.9166969122444578 \\
0.869630351158799 & 0.4864052557384571 & 0.9164094504179578 \\
\hline & 0.1801746003582420 & 0.9157871764888017 \\
\hline
\end{tabular}




\begin{tabular}{|c|c|c|}
\hline 8 & 8 & \\
\hline 2026 & 8654 & \\
\hline 1676622800683614 & 3331341078386448 & \\
\hline & 57 & \\
\hline & 26 & \\
\hline & 574 & \\
\hline & & \\
\hline & & \\
\hline & & \\
\hline 98 & 266 & \\
\hline & & \\
\hline & & \\
\hline 0.3 & 0.6 & 369 \\
\hline & 0.6 & \\
\hline & & \\
\hline & 0. & \\
\hline 0.66 & 0.8 & \\
\hline 1 & 0.0 & 0. \\
\hline & & \\
\hline 32 & 730 & \\
\hline 298 & 344 & \\
\hline 0.3 & & \\
\hline 0.83 & & \\
\hline 0.33 & 0.6 & \\
\hline 0.83 & 0.6 & 0.47 \\
\hline 0.1 & & \\
\hline 0.66 & & \\
\hline 0.16 & 0.8 & \\
\hline 0.6678 & 0.83 & \\
\hline & & \\
\hline & & 0.6454 \\
\hline-0.000600868 & 0.5004 & \\
\hline & & \\
\hline & & \\
\hline 0.8331875199537402 & 0.1665307090051468 & 0.68733920 \\
\hline 37065 & 0.6665 & 0.68 \\
\hline & & \\
\hline 1669520310274434 & 0.3330651183788233 & 0.8129564019299608 \\
\hline 34 & 0.3 & 0.8 \\
\hline & & 0.8 \\
\hline & 0.8330655469295685 & 0813 \\
\hline 0.0008472969098746 & 0.0005379239263053 & 0.8543809137632 \\
\hline 0 & & 0.8 \\
\hline & & \\
\hline
\end{tabular}




$\begin{array}{lll}0.4996945672988957 & 0.4991299277228050 & 0.8543143184721078 \\ 0.3350028960163721 & 0.1663054401926678 & 0.9819690389610712 \\ 0.8339820817318018 & 0.1653510874722732 & 0.9801641712504955 \\ 0.3330909123776841 & 0.6663871269196087 & 0.9803351385870656 \\ 0.8335521228117346 & 0.6666220958523733 & 0.9786455368139547 \\ 0.1643307367877172 & 0.3337671097801522 & 0.3204058505062150\end{array}$

(2.10) $2 \times 2 \times 1$ supercell with the typical snapshots chosen from ab initio molecular dynamics simulations Snapshot: 2.5 ps $(-825.078 \mathrm{eV})$

$\begin{array}{lll}0.17585612 & 0.00884191 & 0.08092728 \\ 0.33388650 & 0.32461342 & 0.08114028 \\ 0.49748034 & 0.18265400 & 0.07809961 \\ 0.67470423 & 0.01066992 & 0.08822624 \\ 0.83295344 & 0.30551373 & 0.09068044 \\ 0.98366117 & 0.16983677 & 0.08147168 \\ 0.16706411 & 0.50756958 & 0.08150383 \\ 0.32576316 & 0.80998693 & 0.08683848 \\ 0.47687077 & 0.65924328 & 0.07958930 \\ 0.69103157 & 0.51823515 & 0.08920515 \\ 0.84510483 & 0.82336558 & 0.09206845 \\ 0.97921486 & 0.65436593 & 0.07785480 \\ 0.14495510 & 0.17137759 & 0.25844036 \\ 0.00413481 & 0.33347985 & 0.26269552 \\ 0.35106401 & 0.99696688 & 0.25609387 \\ 0.64235217 & 0.14922517 & 0.25178375 \\ 0.50498349 & 0.36633057 & 0.24778339 \\ 0.83956656 & 0.98211519 & 0.24896980 \\ 0.14620373 & 0.65132104 & 0.24618031 \\ 0.01461429 & 0.86070000 & 0.25113062 \\ 0.31159787 & 0.47088911 & 0.25893178 \\ 0.64405697 & 0.64637780 & 0.24957781 \\ 0.48782130 & 0.83579688 & 0.24578767 \\ 0.84092923 & 0.48735427 & 0.24098212 \\ 0.02169276 & 0.18813898 & 0.41223132 \\ 0.31307764 & 0.33526963 & 0.41236007 \\ 0.16833202 & 0.47954944 & 0.41320752 \\ 0.50460174 & 0.15831579 & 0.42051136 \\ 0.81758971 & 0.33054555 & 0.41810964 \\ 0.66402315 & 0.48976000 & 0.41535116 \\ 0.00959369 & 0.67955677 & 0.41613738 \\ 0.31805361 & 0.82628240 & 0.41831361 \\ 0.15728182 & 0.97286355 & 0.41365101 \\ 0.51064235 & 0.68295234 & 0.41825138 \\ 0.82020852 & 0.84171728 & 0.41260069\end{array}$




\begin{tabular}{|c|c|c|}
\hline 41 & 631 & 0 \\
\hline 0.49431305 & 0.31586239 & 0.59092787 \\
\hline 18485279 & 15434432 & 0.57893633 \\
\hline 32268760 & 0.99659602 & 9092351 \\
\hline 99612666 & 0.31623393 & 0.58234500 \\
\hline 0.68172443 & 0.16395973 & 0.57905919 \\
\hline 525 & 52 & \\
\hline 3608 & 0.83243973 & \\
\hline 0.17377668 & 0.65386446 & 17503 \\
\hline 0.33794644 & 0.51415223 & 0.57839676 \\
\hline 0.98050894 & 0.81106718 & \\
\hline 0.674 & 0.66418958 & 0.579 \\
\hline 0.82134401 & 0.50258250 & 0.59047585 \\
\hline 0.33328639 & 0.34626505 & 0.73233375 \\
\hline 864 & 4473 & \\
\hline 0.99253100 & 0949 & 0.75 \\
\hline 0.84430345 & 0.33471438 & 0.75679710 \\
\hline 65768050 & 0.99682324 & 0.74894321 \\
\hline 8 & 0.1 & \\
\hline 0.35292128 & 0.85059966 & 0.74835460 \\
\hline 0.15136459 & 0.50897673 & 0.74446329 \\
\hline 0.00594010 & 0.65704690 & 0.74 \\
\hline 0.85669617 & 0.85815969 & 0.75280396 \\
\hline 0.66114067 & 0.50779323 & 0.75137896 \\
\hline 0.49962436 & 0.65545903 & 0.74546922 \\
\hline 0.31686253 & 0.48955597 & 0.91638087 \\
\hline 0.16699548 & 0.20000024 & 0.91388852 \\
\hline 0.02328069 & 0.34421851 & 0.91516596 \\
\hline 0.81270636 & 0.49073775 & 0.91293839 \\
\hline 67694310 & 0.18314138 & 0.92249427 \\
\hline 0.51080960 & 0.34181435 & 0.90749726 \\
\hline 0.32229500 & 0.99261710 & 0.91272761 \\
\hline 16334335 & 0.68452137 & 0.91370426 \\
\hline 0.01868929 & 0.84458552 & 0.92000796 \\
\hline 0.81397674 & 0.98492404 & 0.92718283 \\
\hline 0.67592098 & 0.69013966 & 0.90648687 \\
\hline 0.50661316 & 0.82794208 & 0.91194692 \\
\hline 0.16743247 & 0.33626300 & 0.01954131 \\
\hline 0.68196304 & 0.33345714 & 0.02293049 \\
\hline 0.16652475 & 0.83546377 & 0.02680870 \\
\hline 0.67096872 & 0.84665925 & 0.01773318 \\
\hline 0.00756590 & 0.00907835 & 0.14584326 \\
\hline 0.49059146 & 0.00681190 & 0.14334291 \\
\hline 0.98938 & 0.493498 & 0.1420 \\
\hline
\end{tabular}




$\begin{array}{lll}0.50608520 & 0.50545118 & 0.13585867 \\ 0.33063458 & 0.16975524 & 0.19177845 \\ 0.81507644 & 0.14711784 & 0.19205237 \\ 0.31852508 & 0.65013567 & 0.19128504 \\ 0.84137917 & 0.67808572 & 0.19199125 \\ 0.65834986 & 0.34213999 & 0.31308434 \\ 0.17199341 & 0.83442911 & 0.31643207 \\ 0.66395851 & 0.82928510 & 0.31361609 \\ 0.00480246 & 0.00067008 & 0.34734512 \\ 0.49559587 & 0.99682971 & 0.35554662 \\ 0.98982360 & 0.49170400 & 0.35503510 \\ 0.48825364 & 0.49700468 & 0.34597413 \\ 0.33462133 & 0.15226739 & 0.48350219 \\ 0.83312937 & 0.16488832 & 0.47683849 \\ 0.33171432 & 0.66118700 & 0.48212335 \\ 0.83619137 & 0.67538608 & 0.47846168 \\ 0.17895476 & 0.33522847 & 0.52744813 \\ 0.67215454 & 0.33639140 & 0.51952943 \\ 0.16825232 & 0.82557460 & 0.52034377 \\ 0.65604065 & 0.82324299 & 0.51540806 \\ 0.99901853 & 0.99710990 & 0.64049337 \\ 0.50571017 & 0.00105936 & 0.64315332 \\ 0.00147239 & 0.49254364 & 0.65081017 \\ 0.51307753 & 0.50027376 & 0.64537270 \\ 0.33584000 & 0.16386382 & 0.69474068 \\ 0.83480284 & 0.16504366 & 0.68966994 \\ 0.32790337 & 0.66260708 & 0.67970157 \\ 0.84074659 & 0.66985223 & 0.68529906 \\ 0.17260614 & 0.34164165 & 0.80893274 \\ 0.67104663 & 0.33523891 & 0.80831482 \\ 0.17520082 & 0.83786558 & 0.81134606 \\ 0.66176142 & 0.83570104 & 0.81218861 \\ 0.00752384 & 0.00430544 & 0.85696028 \\ 0.50769450 & 0.01171366 & 0.85598837 \\ 0.99595386 & 0.50026374 & 0.86530918 \\ 0.50603145 & 0.50737798 & 0.85003185 \\ 0.33477890 & 0.17054352 & 0.98151268 \\ 0.85004765 & 0.17465437 & 0.98234596 \\ 0.32829189 & 0.67030750 & 0.97862270 \\ 0.14939059 & 0.32323955 & 0.32162576\end{array}$

Snapshot: 5 ps (-825.401 eV)

$\begin{array}{lll}0.18512782 & 0.00790973 & 0.08669184\end{array}$ 


\begin{tabular}{lll}
0.33582585 & 0.31593845 & 0.08569888 \\
0.49607886 & 0.16984256 & 0.07761256 \\
0.67677887 & 0.00892950 & 0.08284409 \\
0.83102553 & 0.32112159 & 0.07691507 \\
0.97982687 & 0.17720941 & 0.08251656 \\
0.19185958 & 0.52235206 & 0.08206264 \\
0.34002402 & 0.82443064 & 0.08111399 \\
0.49496765 & 0.67129539 & 0.09497188 \\
0.67493798 & 0.51537406 & 0.09274184 \\
0.82339240 & 0.81277838 & 0.07569581 \\
0.98301615 & 0.66542368 & 0.09062766 \\
0.16945379 & 0.18662380 & 0.25948683 \\
0.03330833 & 0.35455786 & 0.25064236 \\
0.34151768 & 0.99555358 & 0.24386336 \\
0.66582157 & 0.14578102 & 0.25929099 \\
0.49459439 & 0.34314159 & 0.25326180 \\
0.83689111 & 0.00305711 & 0.24998919 \\
0.15573965 & 0.65692122 & 0.24895344 \\
0.00218598 & 0.84972017 & 0.25606046 \\
0.33797127 & 0.49495535 & 0.25919171 \\
0.65399654 & 0.65199042 & 0.25744076 \\
0.50352628 & 0.85356196 & 0.26006387 \\
0.84380512 & 0.49736950 & 0.24639389 \\
0.00965625 & 0.18186078 & 0.41769529 \\
0.30219652 & 0.33828251 & 0.40888128 \\
0.16913914 & 0.47341336 & 0.40954061 \\
0.51285299 & 0.18070908 & 0.41784392 \\
0.81905232 & 0.33619676 & 0.41173404 \\
0.66801983 & 0.49255648 & 0.41468310 \\
0.02080841 & 0.68331861 & 0.41812295 \\
0.31756191 & 0.82092406 & 0.40670571 \\
0.18141226 & 0.99562912 & 0.41202071 \\
0.50697502 & 0.68834241 & 0.41473700 \\
0.81908458 & 0.83337822 & 0.41647049 \\
0.65568532 & 0.98525759 & 0.42103799 \\
0.48169363 & 0.31462108 & 0.57585645 \\
0.18161620 & 0.16151109 & 0.58157617 \\
0.32035621 & 0.01277086 & 0.58447419 \\
0.98418719 & 0.31881154 & 0.58571353 \\
0.323380264 & 0.16122980 & 0.58153919 \\
& 0.01034325 & 0.57631642 \\
\hline & 0.51126280 & 0.58088408 \\
\hline
\end{tabular}




\begin{tabular}{lll}
0.97785675 & 0.81580133 & 0.57940205 \\
0.68096954 & 0.67824961 & 0.58454364 \\
0.83826662 & 0.51541340 & 0.58371277 \\
0.34855141 & 0.34285095 & 0.74820350 \\
0.14759216 & 0.99749320 & 0.75627040 \\
0.00561658 & 0.16443006 & 0.74413972 \\
0.83986518 & 0.34292184 & 0.75218848 \\
0.65274807 & 0.99163952 & 0.75301790 \\
0.48855526 & 0.14829950 & 0.74900236 \\
0.35688356 & 0.85619826 & 0.74924141 \\
0.15835102 & 0.49914110 & 0.75347027 \\
0.99584736 & 0.65307142 & 0.75786724 \\
0.84806377 & 0.84940953 & 0.74258417 \\
0.65374197 & 0.49908601 & 0.73863907 \\
0.49527393 & 0.63642245 & 0.74877184 \\
0.32457125 & 0.47594722 & 0.92363754 \\
0.16198206 & 0.17233415 & 0.91776879 \\
0.01630873 & 0.32680417 & 0.90791769 \\
0.81564717 & 0.49050388 & 0.91080803 \\
0.67068971 & 0.18085564 & 0.90898848 \\
0.50578587 & 0.32337629 & 0.91349802 \\
0.32706703 & 0.97746387 & 0.91817633 \\
0.17115096 & 0.67799426 & 0.91652788 \\
0.01419364 & 0.82541935 & 0.91927340 \\
0.82494976 & 0.98568949 & 0.91450239 \\
0.66512207 & 0.67099817 & 0.91573675 \\
0.51873527 & 0.83251500 & 0.92016506 \\
0.17940621 & 0.33441814 & 0.01890754 \\
0.67251654 & 0.33172471 & 0.01146477 \\
0.16816645 & 0.83263229 & 0.02408095 \\
0.65943655 & 0.83205191 & 0.02563750 \\
0.99849382 & 0.00581375 & 0.14386904 \\
0.50160812 & 0.00453963 & 0.14205083 \\
0.99773258 & 0.50098145 & 0.14072398 \\
0.50242695 & 0.50451471 & 0.15459907 \\
0.32815729 & 0.16442545 & 0.19011053 \\
0.83768648 & 0.16213616 & 0.18873101 \\
0.33348140 & 0.66506736 & 0.18460875 \\
0.83375236 & 0.68231184 & 0.18700952 \\
0.669511 & 0.33412334 & 0.31062789 \\
\hline & 0.83524176 & 0.31071351 \\
\hline
\end{tabular}




$\begin{array}{lll}0.01002767 & 0.51184623 & 0.34876616 \\ 0.49285586 & 0.50958830 & 0.35474330 \\ 0.33573878 & 0.18047061 & 0.48209417 \\ 0.82510891 & 0.16644559 & 0.47558575 \\ 0.32750233 & 0.65339556 & 0.47906069 \\ 0.83395405 & 0.67060218 & 0.48013257 \\ 0.15323020 & 0.32701263 & 0.52760687 \\ 0.67027990 & 0.33362623 & 0.51800855 \\ 0.17378582 & 0.83083074 & 0.51444831 \\ 0.65974621 & 0.83130548 & 0.52311841 \\ 0.99340112 & 0.99602919 & 0.63779117 \\ 0.50482779 & 0.00749091 & 0.65217396 \\ 0.00167892 & 0.50385358 & 0.64969024 \\ 0.50153052 & 0.49732225 & 0.64008146 \\ 0.32387346 & 0.15669331 & 0.68413535 \\ 0.83817394 & 0.16995445 & 0.68897142 \\ 0.33757801 & 0.66819106 & 0.69099167 \\ 0.84035754 & 0.67120201 & 0.68919098 \\ 0.16977608 & 0.33070030 & 0.81336721 \\ 0.65978609 & 0.33648114 & 0.81235462 \\ 0.17061305 & 0.83391044 & 0.81419386 \\ 0.66704327 & 0.81415588 & 0.80936094 \\ 0.00158815 & 0.98784188 & 0.86073324 \\ 0.51063051 & 0.99707982 & 0.85595929 \\ 0.99997117 & 0.50157764 & 0.85671912 \\ 0.49573967 & 0.49725462 & 0.85399582 \\ 0.32947749 & 0.15748536 & 0.98283440 \\ 0.84474325 & 0.16182685 & 0.97712877 \\ 0.32445234 & 0.65328471 & 0.97419623 \\ 0.82188312 & 0.66664458 & 0.98161686 \\ 0.17345380 & 0.34521558 & 0.31548859\end{array}$

Snapshot: 10 ps (-825.276 eV)

$\begin{array}{lll}0.16873256 & 0.01114556 & 0.07749198 \\ 0.32984229 & 0.32651950 & 0.09072321 \\ 0.48082287 & 0.15423550 & 0.08692874 \\ 0.68166476 & 0.00340060 & 0.08384807 \\ 0.82387255 & 0.30725090 & 0.08296979 \\ 0.98861719 & 0.17821122 & 0.08651607 \\ 0.17593583 & 0.50844966 & 0.08571772 \\ 0.33305093 & 0.81170358 & 0.08304064 \\ 0.48789132 & 0.65589261 & 0.07872915 \\ 0.67924807 & 0.51033731 & 0.07593532 \\ 0.83131058 & 0.81966072 & 0.08092707\end{array}$




$\begin{array}{lll}0.99033506 & 0.65977042 & 0.09250399 \\ 0.14553802 & 0.16842157 & 0.26058640 \\ 0.00813259 & 0.34350378 & 0.26283107 \\ 0.34565151 & 0.00868679 & 0.24255573 \\ 0.64301546 & 0.13729047 & 0.25382606 \\ 0.50505728 & 0.35743814 & 0.24789204 \\ 0.84502249 & 0.99903534 & 0.25039979 \\ 0.14456783 & 0.65319128 & 0.25259289 \\ 0.01965224 & 0.87359946 & 0.24643533 \\ 0.32295940 & 0.47583180 & 0.25552692 \\ 0.64957258 & 0.64822429 & 0.24897991 \\ 0.49332898 & 0.83633623 & 0.25238205 \\ 0.84147169 & 0.48029841 & 0.25004773 \\ 0.02655801 & 0.19126136 & 0.41515782 \\ 0.31244704 & 0.33127465 & 0.41318814 \\ 0.16997881 & 0.47545324 & 0.41109243 \\ 0.52004095 & 0.18401556 & 0.41054090 \\ 0.83167271 & 0.34583885 & 0.42226919 \\ 0.65800665 & 0.48583319 & 0.43058384 \\ 0.01739933 & 0.67912471 & 0.41735674 \\ 0.32322496 & 0.83762293 & 0.40742094 \\ 0.17636244 & 0.99491785 & 0.41773721 \\ 0.51099737 & 0.67985036 & 0.41125483 \\ 0.81390276 & 0.83065282 & 0.41947724 \\ 0.67135847 & 0.98463305 & 0.42230813 \\ 0.47460997 & 0.31409470 & 0.58664285 \\ 0.17341389 & 0.16505975 & 0.58348958 \\ 0.34004915 & 0.01706672 & 0.58241530 \\ 0.98981205 & 0.32813761 & 0.58448825 \\ 0.67384023 & 0.17609428 & 0.58557233 \\ 0.83975420 & 0.02223588 & 0.58319261 \\ 0.48460109 & 0.82652671 & 0.58018414 \\ 0.17832127 & 0.66042304 & 0.58174271 \\ 0.32943916 & 0.51620591 & 0.59298110 \\ 0.99594434 & 0.82406215 & 0.57764631 \\ 0.67403213 & 0.66260885 & 0.57547243 \\ 0.83258526 & 0.51943134 & 0.58513004 \\ 0.34722779 & 0.35523026 & 0.75315184 \\ 0.15221405 & 0.00393923 & 0.75344329 \\ 0.346901254 & 0.14839266 & 0.74964163 \\ & 0.84668719 & 0.75355569\end{array}$




\begin{tabular}{lll}
0.15785042 & 0.50994110 & 0.75067737 \\
0.00485131 & 0.65822888 & 0.75132398 \\
0.84336272 & 0.84962695 & 0.74996617 \\
0.64885313 & 0.50306086 & 0.75506852 \\
0.51198181 & 0.66813369 & 0.73832526 \\
0.31988443 & 0.48955253 & 0.91199855 \\
0.16601818 & 0.17421356 & 0.91625796 \\
0.01612739 & 0.33507745 & 0.92328192 \\
0.81370823 & 0.48335850 & 0.90857197 \\
0.67304517 & 0.19759709 & 0.91313789 \\
0.50038453 & 0.32986330 & 0.92450063 \\
0.31957729 & 0.97925678 & 0.91491014 \\
0.16583244 & 0.68490668 & 0.92629261 \\
0.00424336 & 0.82729620 & 0.91903241 \\
0.81807785 & 0.99713183 & 0.91236096 \\
0.66301803 & 0.67687393 & 0.91943662 \\
0.52228294 & 0.83550199 & 0.91607587 \\
0.16632876 & 0.33503975 & 0.02508180 \\
0.65298906 & 0.31725693 & 0.01942798 \\
0.16045357 & 0.82626509 & 0.02775963 \\
0.66537356 & 0.82648604 & 0.02744207 \\
0.98500325 & 0.99181056 & 0.14803128 \\
0.49748697 & 0.98853107 & 0.15092600 \\
0.99289576 & 0.48907001 & 0.14086792 \\
0.51110722 & 0.50179704 & 0.14013883 \\
0.32423789 & 0.17368087 & 0.18353640 \\
0.83776171 & 0.16184919 & 0.18703258 \\
0.31870904 & 0.65291588 & 0.19052547 \\
0.83222121 & 0.66209083 & 0.18956076 \\
0.66026346 & 0.31743177 & 0.31020100 \\
0.17744284 & 0.84352892 & 0.30314133 \\
0.67033653 & 0.83129118 & 0.31384207 \\
0.00852457 & 0.01085258 & 0.35311202 \\
0.50116625 & 0.00029310 & 0.35363935 \\
0.00264119 & 0.50575632 & 0.35424120 \\
0.50502175 & 0.49955112 & 0.34982640 \\
0.33727233 & 0.17292364 & 0.48196541 \\
0.83586283 & 0.17481702 & 0.47791391 \\
0.32825335 & 0.66513127 & 0.47401237 \\
0.8611737596 & 0.66476996 & 0.46761760 \\
& 0.83460381 & 0.52324668 \\
\hline
\end{tabular}




$\begin{array}{lll}0.00745479 & 0.99744476 & 0.63999099 \\ 0.49068142 & 0.99933774 & 0.65039539 \\ 0.00118075 & 0.50760091 & 0.64529592 \\ 0.50360997 & 0.50180737 & 0.63947942 \\ 0.33511984 & 0.17954488 & 0.69525852 \\ 0.83195319 & 0.16090375 & 0.68511780 \\ 0.33567773 & 0.66941137 & 0.68715240 \\ 0.84013404 & 0.66690137 & 0.69247465 \\ 0.17286077 & 0.32474945 & 0.81967344 \\ 0.68799117 & 0.34199359 & 0.80573610 \\ 0.16051489 & 0.83717342 & 0.82024974 \\ 0.66586293 & 0.83951555 & 0.80993082 \\ 0.99188391 & 0.99978555 & 0.85122638 \\ 0.50711667 & 0.00516544 & 0.85351418 \\ 0.01593819 & 0.50537386 & 0.85121854 \\ 0.50602376 & 0.50118617 & 0.85306124 \\ 0.33199569 & 0.15289710 & 0.97246136 \\ 0.82947182 & 0.16760803 & 0.97686647 \\ 0.32995644 & 0.66338649 & 0.97696705 \\ 0.82657066 & 0.66223506 & 0.98323309 \\ 0.16627408 & 0.32991441 & 0.32329963\end{array}$

Snapshot: 12.7 ps (-824.846 eV)

$\begin{array}{llll}0.17918210 & 0.02178971 & 0.08563096\end{array}$

$\begin{array}{llll}0.33457688 & 0.31509796 & 0.07937293\end{array}$

$\begin{array}{llll}0.47650706 & 0.15533995 & 0.08158409\end{array}$

$\begin{array}{lll}0.67782133 & 0.02401762 & 0.08925022\end{array}$

$\begin{array}{llll}0.85437960 & 0.33707476 & 0.07814408\end{array}$

$\begin{array}{llll}0.98657578 & 0.17625567 & 0.08908163\end{array}$

$\begin{array}{llll}0.18634827 & 0.51196321 & 0.07466695\end{array}$

$\begin{array}{lll}0.33598272 & 0.83038127 & 0.08803499\end{array}$

$\begin{array}{lll}0.48186507 & 0.68060221 & 0.08431651\end{array}$

$\begin{array}{llll}0.68949897 & 0.50945393 & 0.08465216\end{array}$

$\begin{array}{llll}0.83376174 & 0.82511159 & 0.09153791\end{array}$

$\begin{array}{llll}0.98979677 & 0.67356016 & 0.09411385\end{array}$

$\begin{array}{llll}0.17419376 & 0.18732262 & 0.25945031\end{array}$

$\begin{array}{lll}0.02480134 & 0.33991562 & 0.26560699\end{array}$

$\begin{array}{llll}0.35104087 & 0.00619946 & 0.24663216\end{array}$

$\begin{array}{llll}0.65308963 & 0.15682608 & 0.24663598\end{array}$

$\begin{array}{llll}0.50319660 & 0.34555934 & 0.25191160\end{array}$

$\begin{array}{llll}0.84184221 & 0.99699838 & 0.25073756\end{array}$

$\begin{array}{llll}0.16007158 & 0.66103292 & 0.25374611\end{array}$

$\begin{array}{llll}0.01251023 & 0.85427379 & 0.25206113\end{array}$

$\begin{array}{llll}0.33204045 & 0.49656608 & 0.25929000\end{array}$ 


$\begin{array}{lll}0.66699562 & 0.66506745 & 0.25403757 \\ 0.49335843 & 0.84999065 & 0.25811651 \\ 0.85524369 & 0.49803287 & 0.25138012 \\ 0.01364807 & 0.18985979 & 0.41448644 \\ 0.30649750 & 0.33240952 & 0.40883456 \\ 0.16775850 & 0.47427669 & 0.41101300 \\ 0.51789584 & 0.18622499 & 0.41764704 \\ 0.81355895 & 0.33392246 & 0.41286024 \\ 0.66666977 & 0.49844443 & 0.40888662 \\ 0.02206516 & 0.68797116 & 0.42222359 \\ 0.32083830 & 0.83517906 & 0.41604924 \\ 0.16941742 & 0.99650821 & 0.41304292 \\ 0.52783506 & 0.68505096 & 0.42039267 \\ 0.82844828 & 0.84477370 & 0.41592774 \\ 0.67146359 & 0.00340503 & 0.41469798 \\ 0.48925973 & 0.32590643 & 0.57527502 \\ 0.18437536 & 0.16608006 & 0.58017063 \\ 0.33306932 & 0.01382516 & 0.58397391 \\ 0.98753162 & 0.32668208 & 0.58172183 \\ 0.67875920 & 0.15907510 & 0.58468286 \\ 0.84052829 & 0.01287291 & 0.58544776 \\ 0.48700524 & 0.82422108 & 0.58117319 \\ 0.17749695 & 0.66187010 & 0.57825167 \\ 0.32939054 & 0.49968028 & 0.58053027 \\ 0.98524628 & 0.81364957 & 0.58714222 \\ 0.69588717 & 0.67998003 & 0.58336922 \\ 0.82519874 & 0.50839652 & 0.57955524 \\ 0.35110431 & 0.34455904 & 0.75073667 \\ 0.15091687 & 0.99608165 & 0.74493751 \\ 0.99712248 & 0.14413974 & 0.74834387 \\ 0.84163002 & 0.35222658 & 0.73815569 \\ 0.64780544 & 0.99824162 & 0.74882117 \\ 0.49247401 & 0.14566506 & 0.75856169 \\ 0.34661638 & 0.83883817 & 0.75320081 \\ 0.15440184 & 0.48768965 & 0.74623009 \\ 0.98457990 & 0.63570076 & 0.74422921 \\ 0.85585617 & 0.84804241 & 0.74722019 \\ 0.65573812 & 0.51124158 & 0.75005308 \\ 0.49800283 & 0.65717633 & 0.75420875 \\ 0.675527987 & 0.47801197 & 0.91428114 \\ & 0.18183965 & 0.90862655\end{array}$




\begin{tabular}{lll}
0.51714614 & 0.33636618 & 0.92322590 \\
0.31116820 & 0.97997041 & 0.91842815 \\
0.17021399 & 0.67987136 & 0.91188969 \\
0.01748705 & 0.83260201 & 0.91912441 \\
0.81660382 & 0.98164182 & 0.91469199 \\
0.66749488 & 0.68180684 & 0.92125085 \\
0.51872674 & 0.84756959 & 0.91834490 \\
0.16763900 & 0.33514020 & 0.02962665 \\
0.66246841 & 0.33375840 & 0.02014059 \\
0.15795128 & 0.82866771 & 0.03131926 \\
0.65956482 & 0.83762808 & 0.02781950 \\
0.99404116 & 0.00239964 & 0.14972122 \\
0.49743271 & 0.00166720 & 0.15185877 \\
0.00998638 & 0.50854013 & 0.14402056 \\
0.50800860 & 0.52347810 & 0.13748572 \\
0.32582714 & 0.16583674 & 0.18689923 \\
0.83113133 & 0.16950727 & 0.18569699 \\
0.32788845 & 0.66207199 & 0.18786288 \\
0.83300043 & 0.66164170 & 0.18951321 \\
0.66295660 & 0.33285288 & 0.30919544 \\
0.16749857 & 0.83683755 & 0.31659353 \\
0.66458411 & 0.83101441 & 0.31464492 \\
0.99287156 & 0.00902004 & 0.35266487 \\
0.50112379 & 0.00562433 & 0.35786376 \\
0.99744053 & 0.50255962 & 0.35337500 \\
0.49929990 & 0.49739266 & 0.34628911 \\
0.33393527 & 0.17123907 & 0.48225711 \\
0.83239800 & 0.17312285 & 0.48176623 \\
0.33267212 & 0.66380551 & 0.47579788 \\
0.83029447 & 0.66272344 & 0.47592326 \\
0.15937455 & 0.32197352 & 0.51783759 \\
0.65910305 & 0.32988127 & 0.51934449 \\
0.16453052 & 0.83524654 & 0.52307142 \\
0.67544937 & 0.84262342 & 0.51730984 \\
0.99969406 & 0.99850594 & 0.64510871 \\
0.49904568 & 0.98718062 & 0.64720392 \\
0.99904434 & 0.50091630 & 0.64181394 \\
0.50769717 & 0.50378199 & 0.64326584 \\
0.34364568 & 0.16945922 & 0.68830758 \\
0.860588653 & 0.15937283 & 0.68551067 \\
\hline & 0.32552666 & 0.80503987
\end{tabular}




$\begin{array}{lll}0.17204401 & 0.82856615 & 0.81290194 \\ 0.68120414 & 0.83497475 & 0.81050250 \\ 0.99757632 & 0.98831164 & 0.85338198 \\ 0.50075160 & 0.98997527 & 0.85562144 \\ 0.99843723 & 0.48470859 & 0.84146757 \\ 0.49500358 & 0.49755253 & 0.85808446 \\ 0.33237408 & 0.16431158 & 0.98195352 \\ 0.83149638 & 0.17398113 & 0.97534945 \\ 0.33288083 & 0.66594045 & 0.98438226 \\ 0.83621520 & 0.67317302 & 0.98145744 \\ 0.17879208 & 0.34109917 & 0.32243067\end{array}$

Snapshot: 15.5 ps (-825.302 eV)

$\begin{array}{lll}0.17940601 & 0.00912219 & 0.08782599 \\ 0.32692611 & 0.31574615 & 0.08385999 \\ 0.48884938 & 0.16718955 & 0.08797120 \\ 0.66983299 & 0.00171563 & 0.07984027 \\ 0.83894009 & 0.32575911 & 0.08447012 \\ 0.96548634 & 0.14740775 & 0.07929678 \\ 0.19709739 & 0.51931795 & 0.08199334 \\ 0.33608235 & 0.81898231 & 0.07392744 \\ 0.49788365 & 0.65629741 & 0.07826170 \\ 0.67376497 & 0.50988590 & 0.09051826 \\ 0.83056922 & 0.80870281 & 0.08537522 \\ 0.99421832 & 0.67130821 & 0.07308992 \\ 0.15754774 & 0.16839982 & 0.25682619 \\ 0.02391838 & 0.33540385 & 0.25094999 \\ 0.33470222 & 0.00114597 & 0.25289999 \\ 0.66525476 & 0.14790150 & 0.24753472 \\ 0.49810453 & 0.34255742 & 0.25510670 \\ 0.85118622 & 0.99464192 & 0.24942941 \\ 0.14327978 & 0.64404986 & 0.24011942 \\ 0.98591051 & 0.83802182 & 0.25323304 \\ 0.32323247 & 0.46810059 & 0.26191483 \\ 0.64670226 & 0.65539550 & 0.25110562 \\ 0.49307562 & 0.85153182 & 0.25606244 \\ 0.85789078 & 0.49620460 & 0.24584033 \\ 0.02720097 & 0.19316234 & 0.40858367 \\ 0.30696504 & 0.32129244 & 0.41010665 \\ 0.16936044 & 0.47049165 & 0.41500100 \\ 0.51210435 & 0.17890562 & 0.41102847 \\ 0.82299304 & 0.32737281 & 0.40811003 \\ 0.66185268 & 0.48605498 & 0.41490535 \\ 0.01536036 & 0.67410894 & 0.41599645\end{array}$




\begin{tabular}{|c|c|c|}
\hline 12 & 642 & 0. \\
\hline 0.15969444 & 0.97585166 & 0.42514824 \\
\hline 51366337 & 0.67674330 & 0.41812510 \\
\hline 10753 & 0.83841508 & 424 \\
\hline 66075486 & 0.98448804 & 0.41755347 \\
\hline 0.49335432 & 0.32873516 & 0.57712837 \\
\hline 18100000 & 0.17935 & \\
\hline 8737 & 0.01 & 0.58 \\
\hline 0.99032173 & 0.32410051 & 0.58 \\
\hline 0.69092312 & 0.18052480 & 0.57862187 \\
\hline 83893198 & 91686 & 0.58 \\
\hline 0.48616166 & 0.83925343 & 0.57707861 \\
\hline 0.17439028 & 0.66617839 & 0.57996630 \\
\hline 0.33931563 & 0.52284457 & 0.58097969 \\
\hline 0.98 & 0.83 & \\
\hline 0.68202633 & 0.66442517 & 7092 \\
\hline 0.83708604 & 0.51179139 & 0.57838795 \\
\hline 34828001 & 0.34946295 & 0.75081658 \\
\hline 9 & 0.0 & \\
\hline 0.99216749 & 0.14658038 & 0.75231541 \\
\hline 0.85174353 & 0.35370133 & 0.75516642 \\
\hline 0.65961732 & 0.01223874 & 0.7 \\
\hline 0.51540302 & 0.16541161 & 0.74750506 \\
\hline 0.35340448 & 0.85810393 & 0.75034563 \\
\hline 0.16011995 & 0.51762484 & 0.76162094 \\
\hline 0.99753926 & 0.65811172 & 0.74329997 \\
\hline 0.84249715 & 0.84942167 & 0.74849286 \\
\hline 0.65218572 & 0.49856633 & 0.74224288 \\
\hline 0.49653772 & 0.64745832 & 0.74817626 \\
\hline 31988895 & 0.48146615 & 0.90993996 \\
\hline 0.17554969 & 0.19389609 & 0.91662375 \\
\hline 0.01413685 & 0.34802720 & 0.91408312 \\
\hline 81880791 & 0.48315874 & 0.90693086 \\
\hline 0.67067085 & 0.17603265 & 0.91721291 \\
\hline 0.51440552 & 0.33015975 & 0.91777278 \\
\hline 0.32213253 & 0.99700521 & 0.91409893 \\
\hline 0.17503763 & 0.68587025 & 0.91640141 \\
\hline 0.01085283 & 0.83771263 & 0.91030611 \\
\hline 0.81784285 & 0.99273271 & 0.91195516 \\
\hline 0.66481450 & 0.68648670 & 0.91567404 \\
\hline 0.51205661 & 0.83050365 & 0.91073695 \\
\hline 0.15977853 & 0.32998968 & 0.02340619 \\
\hline 0.67048734 & 0.33514244 & 0.020344 \\
\hline 0.172723 & 0.8352086 & 0.01339 \\
\hline
\end{tabular}




\begin{tabular}{lll}
0.67136757 & 0.83081330 & 0.01750048 \\
0.99040769 & 0.98739928 & 0.15340364 \\
0.48711870 & 0.97942958 & 0.14471183 \\
0.00756859 & 0.50988983 & 0.13721000 \\
0.50065779 & 0.49185105 & 0.15024004 \\
0.33830498 & 0.16783824 & 0.19215948 \\
0.83811484 & 0.15952535 & 0.18708097 \\
0.34398637 & 0.65643478 & 0.18783261 \\
0.81556545 & 0.65135710 & 0.19001270 \\
0.66438993 & 0.31582969 & 0.31138028 \\
0.15404514 & 0.81821701 & 0.31035575 \\
0.65182437 & 0.82903964 & 0.31939752 \\
0.00012411 & 0.00380217 & 0.35970947 \\
0.48385665 & 0.99159895 & 0.35337864 \\
0.01636922 & 0.50209036 & 0.34625813 \\
0.49371271 & 0.49426095 & 0.35647160 \\
0.32406000 & 0.15718353 & 0.48295474 \\
0.82497022 & 0.16683663 & 0.47526461 \\
0.33370863 & 0.67530863 & 0.48175725 \\
0.83812207 & 0.66618877 & 0.48093662 \\
0.15938932 & 0.33674254 & 0.52303514 \\
0.66291362 & 0.33825761 & 0.51360277 \\
0.17452854 & 0.84130606 & 0.52064493 \\
0.67202188 & 0.83036499 & 0.52764167 \\
0.00359638 & 0.00988607 & 0.64198252 \\
0.51063371 & 0.01212313 & 0.64374563 \\
0.99539389 & 0.49959027 & 0.64870679 \\
0.49844697 & 0.50501614 & 0.64481116 \\
0.35399215 & 0.18327726 & 0.69594261 \\
0.84133667 & 0.17375313 & 0.68555577 \\
0.33828160 & 0.67002564 & 0.69664590 \\
0.83457589 & 0.67938438 & 0.68896361 \\
0.16129140 & 0.33121657 & 0.81564432 \\
0.67486865 & 0.34303127 & 0.80939714 \\
0.15945364 & 0.83188613 & 0.80979595 \\
0.66595037 & 0.83667449 & 0.80811871 \\
0.00464529 & 0.01106027 & 0.85181146 \\
0.49356276 & 0.99595210 & 0.85130171 \\
0.00913370 & 0.51221664 & 0.85341361 \\
0.3327569 & 0.50438504 & 0.85368720 \\
\hline & 0.16415939 & 0.98377619 \\
\hline & 0.15875073 & 0.98877984 \\
0.66224286 & 0.97711753 \\
\hline & 0.66393232 & 0.97939305
\end{tabular}


Snapshot: 18.5 ps (-825.954 eV)

\begin{tabular}{lll}
0.16976757 & 0.00624469 & 0.07609527 \\
0.32605400 & 0.30667223 & 0.08339607 \\
0.48144855 & 0.16043310 & 0.08288814 \\
0.67957160 & 0.01059871 & 0.08593667 \\
0.82963728 & 0.31390690 & 0.08706621 \\
0.98556334 & 0.16553902 & 0.08618643 \\
0.17464031 & 0.50040147 & 0.08059917 \\
0.32334177 & 0.81416927 & 0.08475583 \\
0.46851506 & 0.65909909 & 0.08524108 \\
0.69523968 & 0.50710124 & 0.08248491 \\
0.82477420 & 0.81502536 & 0.08360552 \\
0.99886545 & 0.67404403 & 0.08968898 \\
0.16362184 & 0.17489037 & 0.25061815 \\
0.00809779 & 0.34735806 & 0.25610714 \\
0.35134328 & 0.99652150 & 0.24657551 \\
0.65605854 & 0.14359002 & 0.25680196 \\
0.50377995 & 0.34844523 & 0.24867954 \\
0.85219569 & 0.99193579 & 0.24729675 \\
0.15115085 & 0.64972807 & 0.24366797 \\
0.00632497 & 0.84633552 & 0.24793852 \\
0.32199225 & 0.48543818 & 0.26200560 \\
0.65514939 & 0.65195326 & 0.24845582 \\
0.49284812 & 0.83469468 & 0.25125964 \\
0.84663594 & 0.48754628 & 0.25038091 \\
0.03321215 & 0.20050932 & 0.40677704 \\
0.31351673 & 0.34090062 & 0.41048351 \\
0.16709596 & 0.47728586 & 0.41143164 \\
0.51690617 & 0.18329795 & 0.42019242 \\
0.83134571 & 0.34688803 & 0.41957313 \\
0.65653014 & 0.48129159 & 0.41523905 \\
0.01392301 & 0.68323362 & 0.41916636 \\
0.32072249 & 0.83684624 & 0.42113627 \\
0.16513105 & 0.99000622 & 0.42389118 \\
0.52365516 & 0.68610102 & 0.41273495 \\
0.82466414 & 0.83646074 & 0.40810645 \\
0.66730859 & 0.98931247 & 0.41854148 \\
0.47760474 & 0.31455396 & 0.58063293 \\
0.18107512 & 0.16478424 & 0.58701436 \\
0.33860112 & 0.02440112 & 0.58363342 \\
0.98729316 & 0.32400147 & 0.58545404 \\
0.67750068 & 0.16856118 & 0.58784348 \\
\hline & & \\
\hline
\end{tabular}




\begin{tabular}{lll}
0.83813731 & 0.02227012 & 0.58138645 \\
0.49566803 & 0.82661449 & 0.58615591 \\
0.18201472 & 0.67455421 & 0.58648439 \\
0.32803510 & 0.51520156 & 0.57425496 \\
0.98378512 & 0.81609845 & 0.58554330 \\
0.69448349 & 0.67505731 & 0.57548051 \\
0.83864774 & 0.51519587 & 0.59142811 \\
0.34455338 & 0.36128782 & 0.73963096 \\
0.14363065 & 0.99320895 & 0.75522585 \\
0.99605237 & 0.15384296 & 0.75855417 \\
0.83965152 & 0.35191432 & 0.75112287 \\
0.65027175 & 0.00516280 & 0.76031754 \\
0.48699604 & 0.15709018 & 0.75530253 \\
0.34468354 & 0.84224543 & 0.75382492 \\
0.15645626 & 0.50252714 & 0.74673260 \\
0.98730718 & 0.64511326 & 0.75395975 \\
0.84352949 & 0.84456087 & 0.74651536 \\
0.65010727 & 0.50225617 & 0.74784394 \\
0.51033100 & 0.67144298 & 0.74745649 \\
0.31872621 & 0.48262335 & 0.91367882 \\
0.17210187 & 0.18404572 & 0.91242932 \\
0.01764825 & 0.32693529 & 0.91958394 \\
0.82757127 & 0.49256810 & 0.91371988 \\
0.67139602 & 0.18303310 & 0.91706166 \\
0.50441637 & 0.33292770 & 0.91017939 \\
0.32190610 & 0.99100831 & 0.91920918 \\
0.15518172 & 0.67272858 & 0.91378528 \\
0.99904692 & 0.82481364 & 0.91136986 \\
0.81309196 & 0.98462807 & 0.91849458 \\
0.66528745 & 0.68638482 & 0.92206150 \\
0.50657393 & 0.83062053 & 0.92051968 \\
0.16550630 & 0.32428186 & 0.02815825 \\
0.66755857 & 0.32356328 & 0.01910064 \\
0.16503196 & 0.83721022 & 0.01130350 \\
0.65207913 & 0.83062126 & 0.02023329 \\
0.99096038 & 0.99149685 & 0.14317397 \\
0.49547634 & 0.99447949 & 0.14429213 \\
0.99472508 & 0.49322352 & 0.14769997 \\
0.50632725 & 0.50377430 & 0.13974135 \\
0.33033756 & 0.16973643 & 0.18253624 \\
\hline & 0.17100164 & 0.19131535 \\
\hline
\end{tabular}




$\begin{array}{lll}0.17020299 & 0.82904500 & 0.31689259 \\ 0.66289054 & 0.83212575 & 0.31497323 \\ 0.00695859 & 0.00077056 & 0.34694896 \\ 0.50796327 & 0.00133236 & 0.34907181 \\ 0.00879304 & 0.50246427 & 0.35109205 \\ 0.50029654 & 0.50004444 & 0.34615701 \\ 0.33362895 & 0.17430072 & 0.47325835 \\ 0.83087651 & 0.17370930 & 0.48503159 \\ 0.34115912 & 0.67588709 & 0.47242625 \\ 0.82627304 & 0.66252024 & 0.46887620 \\ 0.16287956 & 0.33170030 & 0.52226649 \\ 0.66017565 & 0.33037743 & 0.52194381 \\ 0.16883603 & 0.84078031 & 0.52270792 \\ 0.67430743 & 0.84337849 & 0.52210549 \\ 0.99853173 & 0.99548236 & 0.64388064 \\ 0.50556662 & 0.00298946 & 0.65090042 \\ 0.00966671 & 0.51330583 & 0.65195272 \\ 0.50321749 & 0.50315977 & 0.64242360 \\ 0.32327855 & 0.16890201 & 0.68860458 \\ 0.83552840 & 0.17346597 & 0.69481528 \\ 0.34150878 & 0.67594324 & 0.68565463 \\ 0.83626723 & 0.66206321 & 0.68475677 \\ 0.17470288 & 0.34354652 & 0.81519655 \\ 0.65215476 & 0.33090756 & 0.81479099 \\ 0.15349323 & 0.82219153 & 0.81139621 \\ 0.67301348 & 0.83559478 & 0.81424722 \\ 0.00173971 & 0.00436976 & 0.85648733 \\ 0.49970874 & 0.00170620 & 0.85511779 \\ 0.99759092 & 0.48654269 & 0.86086089 \\ 0.51556281 & 0.51280003 & 0.85519144 \\ 0.33244272 & 0.16225619 & 0.97731473 \\ 0.84265793 & 0.16323578 & 0.97683961 \\ 0.32902643 & 0.66242102 & 0.98376796 \\ 0.82457457 & 0.65851880 & 0.98006818 \\ 0.17366147 & 0.33136747 & 0.31847680\end{array}$

Snapshot: 20 ps (-825.172 eV)

$\begin{array}{lll}0.17134157 & 0.01156901 & 0.07893865 \\ 0.33165799 & 0.32753465 & 0.08963277 \\ 0.48441686 & 0.16860203 & 0.09092322 \\ 0.69034870 & 0.02164183 & 0.08254276 \\ 0.83209765 & 0.32155052 & 0.08105524 \\ 0.98706489 & 0.16704272 & 0.08926161 \\ 0.18349230 & 0.52213105 & 0.08361474\end{array}$




\begin{tabular}{lll}
0.34291526 & 0.84247736 & 0.07884360 \\
0.49976993 & 0.65720239 & 0.07589740 \\
0.68257584 & 0.52260443 & 0.07459996 \\
0.83533997 & 0.82931180 & 0.08519401 \\
0.98004052 & 0.66370305 & 0.09551937 \\
0.14798343 & 0.16716475 & 0.26083506 \\
0.00884977 & 0.32310462 & 0.26758095 \\
0.34318586 & 0.99541246 & 0.24241024 \\
0.65074445 & 0.15248167 & 0.25277510 \\
0.49809949 & 0.34097607 & 0.24653805 \\
0.83684413 & 0.98800642 & 0.25082468 \\
0.14135481 & 0.64696791 & 0.25170010 \\
0.00084104 & 0.85374681 & 0.25179149 \\
0.32769258 & 0.46953856 & 0.26624327 \\
0.65703491 & 0.65318056 & 0.24491800 \\
0.51261296 & 0.84534946 & 0.24142890 \\
0.84709595 & 0.49084164 & 0.25269504 \\
0.02968300 & 0.18696933 & 0.41775982 \\
0.30894253 & 0.31736508 & 0.41351258 \\
0.16610840 & 0.47629140 & 0.41386153 \\
0.50863793 & 0.16948409 & 0.40866387 \\
0.82095835 & 0.32227802 & 0.40382582 \\
0.68129308 & 0.50324351 & 0.41314054 \\
0.01008801 & 0.68184037 & 0.42298575 \\
0.31783400 & 0.82972100 & 0.41627537 \\
0.16918515 & 0.97969834 & 0.41694325 \\
0.51572262 & 0.67448811 & 0.41544768 \\
0.83289548 & 0.83776696 & 0.42492731 \\
0.67072631 & 0.98311162 & 0.41280955 \\
0.48148192 & 0.31793497 & 0.58368734 \\
0.18379835 & 0.17366141 & 0.58435673 \\
0.32776255 & 0.02012628 & 0.58925129 \\
0.98981687 & 0.31805472 & 0.58221566 \\
0.68469546 & 0.16977625 & 0.58176374 \\
0.83368852 & 0.00994709 & 0.58441026 \\
0.47999219 & 0.82176362 & 0.56595714 \\
0.16454476 & 0.65294893 & 0.57320435 \\
0.33930733 & 0.51772385 & 0.58702512 \\
0.99782686 & 0.82164148 & 0.59479133 \\
0.989959759 & 0.67225676 & 0.58366176 \\
& 0.50452997 & 0.58195929 \\
\hline & 0.34568412 & 0.75511401 \\
\hline & 0.15378179 & 0.75171630 \\
\hline
\end{tabular}




\begin{tabular}{|c|c|c|}
\hline 449 & 665 & 0 \\
\hline 0.64422294 & 0.99672006 & 0.74100173 \\
\hline 50254829 & .14738215 & 0.74414672 \\
\hline 32693362 & 0.83469017 & 4532470 \\
\hline 14428270 & 0.49392636 & 0.74472386 \\
\hline 0.98633105 & 0.65120737 & 0.75703680 \\
\hline 25 & 0.85 & \\
\hline 09685 & 0.49 & 545 \\
\hline 0.50280019 & 0.64972024 & 0.75200780 \\
\hline 0.31202254 & 0.48257860 & 0.91866511 \\
\hline 0.15849706 & 0.18041647 & \\
\hline 0.01029999 & 0.32937621 & 0.91 \\
\hline 0.81090946 & 0.46605326 & 0.91494923 \\
\hline 0.65738897 & 0.17406925 & 0.92129602 \\
\hline 663 & 7203 & \\
\hline 0.327 & 0.986 & 37340 \\
\hline 0.17034074 & 0.67981523 & 0.91543692 \\
\hline 00708823 & 0.82665375 & 0.91725903 \\
\hline 92 & 0.95 & \\
\hline 0.66960605 & 0.68345178 & 0.91901614 \\
\hline 0.50704569 & 0.82992128 & 0.91382014 \\
\hline 16530174 & 0.33336709 & 0.02 \\
\hline $0.661<$ & 0.33236867 & 0.02640647 \\
\hline 0.15686997 & 0.82771208 & 0.02328372 \\
\hline 0.67071571 & 0.83646967 & 0.01775248 \\
\hline 0.00896437 & 0.00653205 & 0.14332100 \\
\hline 0.50228998 & 0.00290624 & 0.14626041 \\
\hline 0.01070569 & 0.50311052 & 0.15196005 \\
\hline 0.49936629 & 0.49880862 & 0.14446911 \\
\hline 33564976 & 0.17555666 & 0.18937057 \\
\hline 0.82706585 & 0.15692244 & 0.19468229 \\
\hline 0.33913127 & 0.66124204 & 0.18407619 \\
\hline 84262454 & 0.67297015 & 0.18868689 \\
\hline 0.66547517 & 0.32774181 & 0.31296186 \\
\hline 0.17318160 & 0.84004164 & 0.31254919 \\
\hline 0.66881235 & 0.82748550 & 0.31216864 \\
\hline 0.99702135 & 0.00289576 & 0.35077294 \\
\hline 0.49988874 & 0.99011482 & 0.34623634 \\
\hline 0.00900645 & 0.51582042 & 0.35623905 \\
\hline 0.49697079 & 0.50049527 & 0.35574936 \\
\hline 0.33787376 & 0.16638700 & 0.47216536 \\
\hline 0.83515256 & 0.16886595 & 0.47826979 \\
\hline 0.33888383 & 0.66891272 & 0.46701069 \\
\hline 0.835113 & 0.664949 & 0.48636 \\
\hline
\end{tabular}




$\begin{array}{lll}0.16295007 & 0.33524333 & 0.52846431 \\ 0.65738994 & 0.33385274 & 0.51734548 \\ 0.16448721 & 0.83512682 & 0.52009275 \\ 0.66992277 & 0.83759614 & 0.51683598 \\ 0.00786287 & 0.01223013 & 0.64455516 \\ 0.49543758 & 0.99624533 & 0.63151105 \\ 0.99939244 & 0.50196226 & 0.64579464 \\ 0.51072755 & 0.50440034 & 0.64731145 \\ 0.32915879 & 0.17039688 & 0.68950210 \\ 0.83228340 & 0.16424128 & 0.68679343 \\ 0.33011269 & 0.66445482 & 0.68623503 \\ 0.83220763 & 0.67420357 & 0.69557662 \\ 0.15769776 & 0.33488584 & 0.81777249 \\ 0.66374159 & 0.33170327 & 0.80747757 \\ 0.16646475 & 0.83138636 & 0.81138784 \\ 0.67885801 & 0.83279452 & 0.81050215 \\ 0.00069488 & 0.99982689 & 0.85569421 \\ 0.50702082 & 0.99962376 & 0.84941855 \\ 0.00079486 & 0.50271098 & 0.85576573 \\ 0.49221936 & 0.49949672 & 0.86095242 \\ 0.32781442 & 0.15262706 & 0.97315150 \\ 0.83321711 & 0.16952682 & 0.98240282 \\ 0.33728061 & 0.66684753 & 0.98228540 \\ 0.82676255 & 0.66287017 & 0.97786627 \\ 0.16337180 & 0.33638228 & 0.32534078\end{array}$

Snapshot: 22.5 ps (-825.244 eV)

$\begin{array}{lll}0.18196723 & 0.02491843 & 0.08426174 \\ 0.31843358 & 0.30603536 & 0.08845890 \\ 0.48494586 & 0.16494435 & 0.08041285 \\ 0.68009755 & 0.01443468 & 0.08601247 \\ 0.84446344 & 0.31966791 & 0.07844421 \\ 0.00625757 & 0.17699122 & 0.08661706 \\ 0.17928149 & 0.51432447 & 0.07953476 \\ 0.32201271 & 0.81752742 & 0.08740380 \\ 0.48671638 & 0.67168147 & 0.08369571 \\ 0.68298010 & 0.51007829 & 0.07654659 \\ 0.83352438 & 0.82308251 & 0.08465061 \\ 0.99150396 & 0.68018215 & 0.08786839 \\ 0.15936463 & 0.16765589 & 0.25782481 \\ 0.02340661 & 0.33622983 & 0.25920651 \\ 0.34237855 & 0.99519535 & 0.24832053 \\ 0.64733619 & 0.14256230 & 0.25761560 \\ 0.48401687 & 0.33293938 & 0.26027882\end{array}$




\begin{tabular}{|c|c|c|}
\hline 523 & 4399 & 0.2 \\
\hline 0.15561263 & 0.65263330 & 0.24818783 \\
\hline 0.99621777 & .84921139 & 0.25114078 \\
\hline 32328220 & 0.47784719 & 6206185 \\
\hline 65133979 & 0.64731011 & 0.24846662 \\
\hline 0.49530298 & 0.84027910 & 0.24936781 \\
\hline 0.84291694 & 0.49332956 & 918 \\
\hline 32054 & 0.19409022 & 0.41 \\
\hline 0.30528631 & 0.32045073 & 0.40410746 \\
\hline 16531917 & 0.47546366 & 0.41109408 \\
\hline 51673911 & 0142 & 319 \\
\hline 0.82129321 & 0.32958951 & 0.41141669 \\
\hline 0.67154233 & 0.48429847 & 0.41418338 \\
\hline 0.02266403 & 0.68050878 & 0.41260681 \\
\hline 9252 & 0.82699043 & 0.40 \\
\hline 0.16018481 & 0.98150225 & 7057 \\
\hline 0.52231843 & 0.67931439 & 0.41500018 \\
\hline 81853604 & 0.82433932 & 0.41397637 \\
\hline 0.667 & 0.98 & \\
\hline 0.48333438 & 0.33085677 & 0.57549986 \\
\hline 0.18749750 & 0.17493905 & 0.58202767 \\
\hline 0.34065928 & 0.02084307 & 0.586 \\
\hline 0.98181844 & 0.32133009 & 0.57 \\
\hline 0.66753442 & 0.16783942 & 0.58662739 \\
\hline 0.83566040 & 0.01767125 & 0.57362916 \\
\hline 48876697 & 0.81317749 & 0.58422931 \\
\hline 18092425 & 0.6758 & 88838 \\
\hline 0.33318565 & 0.53296323 & 0.57994954 \\
\hline 0.97942176 & 0.81505181 & 0.57838355 \\
\hline 69106641 & 0.67111907 & 0.57873916 \\
\hline 0.83202732 & 0.50885015 & 0.57866309 \\
\hline 0.34183676 & 0.34398337 & 0.74440866 \\
\hline 0.15031889 & 0.00951746 & 0.74348679 \\
\hline 0.00334368 & 0.15930129 & 0.74632322 \\
\hline 0.84013519 & 0.34240061 & 0.75155634 \\
\hline 0.65104220 & 0.00103274 & 0.75041713 \\
\hline 0.49635229 & 0.16398041 & 0.75540440 \\
\hline 0.35623001 & 0.84952939 & 0.74968338 \\
\hline 0.14653850 & 0.50105272 & 0.75714055 \\
\hline 0.99895154 & 0.66263431 & 0.74819777 \\
\hline 0.85212580 & 0.85573409 & 0.74434094 \\
\hline 0.66447191 & 0.52097284 & 0.76327013 \\
\hline 0.49701674 & 0.65030717 & 0.7464927 \\
\hline 0.323276 & 0.481417 & 0.91852 \\
\hline
\end{tabular}




\begin{tabular}{lll}
0.16571337 & 0.17366462 & 0.91077730 \\
0.00530484 & 0.32008943 & 0.91534938 \\
0.81535257 & 0.48189176 & 0.92485934 \\
0.65498561 & 0.17635181 & 0.91637180 \\
0.51194497 & 0.33594523 & 0.91683268 \\
0.31179163 & 0.98775067 & 0.91187390 \\
0.16860893 & 0.68409236 & 0.91093538 \\
0.00793791 & 0.82777233 & 0.91602422 \\
0.82072461 & 0.99136242 & 0.91217643 \\
0.66876476 & 0.69080970 & 0.91636063 \\
0.51241599 & 0.83218394 & 0.90941301 \\
0.17813584 & 0.33669285 & 0.01957780 \\
0.66687150 & 0.33474023 & 0.02551457 \\
0.15743385 & 0.83731978 & 0.02084833 \\
0.66342126 & 0.83175700 & 0.02175390 \\
0.99878671 & 0.00064981 & 0.14666885 \\
0.49046839 & 0.99779032 & 0.14332368 \\
0.00493849 & 0.49434368 & 0.13961731 \\
0.49607193 & 0.49154434 & 0.14358655 \\
0.32890408 & 0.16711822 & 0.19364937 \\
0.83473874 & 0.16171072 & 0.18685065 \\
0.33010797 & 0.65368376 & 0.19042654 \\
0.83581765 & 0.66932470 & 0.18764970 \\
0.66294387 & 0.32721333 & 0.31523525 \\
0.15994021 & 0.82108332 & 0.31541910 \\
0.66315392 & 0.82728873 & 0.30727306 \\
0.99336057 & 0.99350848 & 0.35463448 \\
0.49689172 & 0.99529979 & 0.35022727 \\
0.99151911 & 0.48564595 & 0.35226692 \\
0.50504947 & 0.49625019 & 0.35066027 \\
0.32820212 & 0.16546155 & 0.48182408 \\
0.82159725 & 0.16885445 & 0.47772025 \\
0.34201901 & 0.67578227 & 0.47549934 \\
0.84359940 & 0.66568627 & 0.47407949 \\
0.16873614 & 0.32743138 & 0.51499179 \\
0.67704427 & 0.34411867 & 0.52939482 \\
0.17946074 & 0.83986251 & 0.52732775 \\
0.67402501 & 0.83903511 & 0.52677364 \\
0.01168237 & 0.99975209 & 0.63809145 \\
\hline & 0.99573660 & 0.65035413 \\
\hline
\end{tabular}




$\begin{array}{lll}0.32935826 & 0.66446027 & 0.69027415 \\ 0.83587477 & 0.67542576 & 0.68929347 \\ 0.17499039 & 0.33519237 & 0.81508646 \\ 0.66385547 & 0.33564398 & 0.81673625 \\ 0.16485313 & 0.83004893 & 0.80677743 \\ 0.66064285 & 0.83472226 & 0.81182665 \\ 0.99459577 & 0.00466630 & 0.84708344 \\ 0.49727456 & 0.00883192 & 0.85137098 \\ 0.99382771 & 0.49123817 & 0.85466485 \\ 0.50855528 & 0.50611068 & 0.85841844 \\ 0.33709003 & 0.17009646 & 0.98157574 \\ 0.83098533 & 0.16292839 & 0.98362700 \\ 0.33928046 & 0.67161579 & 0.98273403 \\ 0.82976385 & 0.66713843 & 0.98050512 \\ 0.16542110 & 0.33055964 & 0.32075262\end{array}$

Snapshot: 25.5 ps (-825.534 eV)

$\begin{array}{lll}0.17212215 & 0.01072334 & 0.08352400 \\ 0.32240097 & 0.31643663 & 0.08772875 \\ 0.49009843 & 0.17002626 & 0.07823495 \\ 0.68114099 & 0.01933978 & 0.07803447 \\ 0.83099241 & 0.31940795 & 0.08491700 \\ 0.97701647 & 0.15618271 & 0.08180888 \\ 0.16961258 & 0.50324052 & 0.08594917 \\ 0.34057190 & 0.83026033 & 0.07398639 \\ 0.49023550 & 0.66021644 & 0.07417264 \\ 0.68610672 & 0.51622289 & 0.08085262 \\ 0.82501055 & 0.81707296 & 0.08465895 \\ 0.98613387 & 0.66852006 & 0.08689984 \\ 0.15184231 & 0.16376770 & 0.25836780 \\ 0.01212476 & 0.33878781 & 0.26326499 \\ 0.34550622 & 0.99662650 & 0.24342682 \\ 0.66155140 & 0.15983641 & 0.25008143 \\ 0.49941877 & 0.34517609 & 0.24914825 \\ 0.84867703 & 0.98817391 & 0.25068458 \\ 0.14859219 & 0.64561992 & 0.24588850 \\ 0.00523776 & 0.84806254 & 0.24954118 \\ 0.32143255 & 0.47350381 & 0.26521814 \\ 0.64369323 & 0.64905537 & 0.24672263 \\ 0.52323640 & 0.86101911 & 0.24438890 \\ 0.85182795 & 0.49599690 & 0.24576363 \\ 0.02286563 & 0.19129396 & 0.40284632 \\ 0.30984356 & 0.33064606 & 0.41414995 \\ 0.16821220 & 0.47622972 & 0.41823158\end{array}$




\begin{tabular}{|c|c|c|}
\hline 638 & 6275 & $0.4151777 €$ \\
\hline 0.81323992 & 0.33936103 & 0.41946432 \\
\hline 65610896 & 0.47936477 & 0.42390292 \\
\hline 62234 & 0.68344440 & 2884 \\
\hline 32523290 & 0.82810763 & 0.40296495 \\
\hline 0.17406232 & 0.99214592 & 0.40825246 \\
\hline 435 & 568 & 11 \\
\hline 4402 & 0.83263510 & 0.4 \\
\hline 0.66520003 & 0.98127516 & 0.41228260 \\
\hline 0.48990546 & 0.32658183 & 0.58869477 \\
\hline 7330 & 0.17066976 & 0.580 \\
\hline 2175 & 0.00549140 & 0.58 \\
\hline 0.97434498 & 0.31553929 & 0.57948085 \\
\hline 0.67589925 & 0.15742828 & 0.57765512 \\
\hline 0.84 & $0.01 \mathrm{c}$ & 0. \\
\hline 0.49902138 & 0.82278952 & 20339 \\
\hline 0.19119227 & 0.67631703 & 0.58518185 \\
\hline 33258256 & 0.50962661 & 0.58551136 \\
\hline 0.99 & 0.8 & \\
\hline 0.68157487 & 0.67571248 & 0.58894079 \\
\hline 0.84108680 & 0.52214534 & 0.58627481 \\
\hline 0.33827889 & 0.34966422 & 0.7 \\
\hline 0.16519896 & 0.00505717 & 0.75 \\
\hline 0.00746885 & 0.15288348 & 0.75060532 \\
\hline 0.84059454 & 0.35109727 & 0.74236219 \\
\hline 0.65361972 & 0.00692473 & 0.74951630 \\
\hline 0.50217391 & 0.15718365 & 0.74329732 \\
\hline 0.34657144 & 0.84997036 & 0.74623581 \\
\hline 0.16399620 & 0.50612259 & 0.75186890 \\
\hline 01023367 & 0.65644740 & 0.75172191 \\
\hline 0.84755744 & 0.83888319 & 0.74818480 \\
\hline 0.64935645 & 0.50541303 & 0.75365730 \\
\hline 50080310 & 0.65583919 & 0.75273176 \\
\hline 0.31640359 & 0.48674606 & 0.91932448 \\
\hline 0.16632129 & 0.18537228 & 0.92390333 \\
\hline 0.01707252 & 0.34260792 & 0.91644850 \\
\hline 0.80851611 & 0.48035928 & 0.90992031 \\
\hline 0.66600682 & 0.18249495 & 0.92318458 \\
\hline 0.50628499 & 0.32722635 & 0.91026569 \\
\hline 0.31128264 & 0.99071803 & 0.91545590 \\
\hline 0.15630910 & 0.67041939 & 0.92268643 \\
\hline 0.01319027 & 0.82483277 & 0.91551794 \\
\hline 0.82501511 & 0.99111846 & 0.91604369 \\
\hline 0.6611978 & 0.679387 & 0.91790 \\
\hline
\end{tabular}




\begin{tabular}{|c|c|c|}
\hline 703 & 727 & 0. \\
\hline 0.15612029 & 0.32543456 & 0.02554691 \\
\hline 67062207 & 0.33737360 & 0.02209888 \\
\hline 16635128 & 0.83123266 & 695430 \\
\hline 67588463 & 0.83184775 & 0.01693917 \\
\hline 0.99949955 & 0.99571206 & 0.14456449 \\
\hline 0.502 & 20 & 45 \\
\hline 1424 & 6724 & 0 \\
\hline 0.50720448 & 0.50992381 & 2966 \\
\hline 0.32874430 & 0.17323125 & 0.19176413 \\
\hline 0.83166249 & 925 & 0.18 \\
\hline 0.34133595 & 0.66586388 & 0.18414406 \\
\hline 0.83570931 & 0.66758885 & 0.19314046 \\
\hline 0.66523946 & 0.33976281 & 0.31341069 \\
\hline 412 & 0.823 & \\
\hline 0.67362319 & 0.82816211 & 0.32 \\
\hline 0.99998022 & 0.99597633 & 0.35501245 \\
\hline 51117251 & 0.00040200 & 0.34419344 \\
\hline 0.00 & 0.4 & \\
\hline 0.48951643 & 0.50263373 & 0.35611932 \\
\hline 0.33756405 & 0.17069914 & 0.47654135 \\
\hline 84539607 & 0.180 & 0.47778744 \\
\hline 0.33292030 & 0.67028544 & 0.47 \\
\hline 0.83722772 & 0.65961723 & 0.48374538 \\
\hline 0.17198512 & 0.34160319 & 0.52838830 \\
\hline 0.65856720 & 0.32305802 & 0.52881775 \\
\hline 16962996 & 0.83551919 & 0.51500444 \\
\hline 0.66905886 & 0.85011864 & 0.52302740 \\
\hline 0.00764872 & 0.00258393 & 0.63872827 \\
\hline 49844071 & 0.99598052 & 0.64364808 \\
\hline 0.99981003 & 0.49453827 & 0.65074590 \\
\hline 0.50299577 & 0.51154488 & 0.65714618 \\
\hline 33279238 & 0.16963817 & 0.69112907 \\
\hline 0.83700403 & 0.15736025 & 0.68856268 \\
\hline 0.32839942 & 0.66482839 & 0.68633200 \\
\hline 0.83832587 & 0.65933171 & 0.68418106 \\
\hline 0.16167586 & 0.32723474 & 0.81665143 \\
\hline 0.65525987 & 0.33512971 & 0.81282768 \\
\hline 0.16331332 & 0.82695431 & 0.82041742 \\
\hline 0.65500413 & 0.83529093 & 0.81174605 \\
\hline 0.00142063 & 0.99497783 & 0.85421496 \\
\hline 0.50259471 & 0.01896643 & 0.85402446 \\
\hline 0.99607107 & 0.49933744 & 0.85317415 \\
\hline 0.492162 & 0.492008 & 0.85482 \\
\hline
\end{tabular}




$\begin{array}{lll}0.32601057 & 0.16418654 & 0.98113451 \\ 0.83176380 & 0.17101870 & 0.97537760 \\ 0.33072672 & 0.66815770 & 0.97697149 \\ 0.82687880 & 0.66277914 & 0.97316331 \\ 0.16067165 & 0.32682058 & 0.32246060\end{array}$

Snapshot: 30 ps (-825.711 eV)

$\begin{array}{lll}0.17022341 & 0.01024737 & 0.08621063\end{array}$

$\begin{array}{llll}0.31682165 & 0.30102111 & 0.08522479\end{array}$

$\begin{array}{llll}0.49704204 & 0.17034997 & 0.08474488\end{array}$

$\begin{array}{lll}0.67686682 & 0.00791558 & 0.07693252\end{array}$

$\begin{array}{llll}0.83226601 & 0.31435942 & 0.09164483\end{array}$

$\begin{array}{lll}0.97467327 & 0.16389136 & 0.08605382\end{array}$

$\begin{array}{llll}0.18267893 & 0.51955465 & 0.08162889\end{array}$

$\begin{array}{llll}0.33557966 & 0.81844538 & 0.08196568\end{array}$

$\begin{array}{llll}0.48355306 & 0.66715110 & 0.08684447\end{array}$

$\begin{array}{llll}0.69218268 & 0.51540084 & 0.08949459\end{array}$

$\begin{array}{llll}0.83291023 & 0.81533714 & 0.08003974\end{array}$

$\begin{array}{llll}0.98802950 & 0.66972680 & 0.07750533\end{array}$

$\begin{array}{llll}0.14972080 & 0.15809687 & 0.25943069\end{array}$

$\begin{array}{llll}0.01834010 & 0.34114827 & 0.26006787\end{array}$

$\begin{array}{llll}0.34812447 & 0.99575293 & 0.24794654\end{array}$

$\begin{array}{lll}0.66570560 & 0.15843032 & 0.25214672\end{array}$

$\begin{array}{llll}0.49567243 & 0.33980492 & 0.24963736\end{array}$

$\begin{array}{llll}0.84286636 & 0.99293546 & 0.23760992\end{array}$

$\begin{array}{llll}0.14925985 & 0.65948404 & 0.24549250\end{array}$

$\begin{array}{llll}0.00123508 & 0.85272041 & 0.24877140\end{array}$

$\begin{array}{llll}0.32256043 & 0.47228548 & 0.25358726\end{array}$

$\begin{array}{llll}0.64793802 & 0.65501000 & 0.26416597\end{array}$

$\begin{array}{llll}0.50730572 & 0.85168030 & 0.25400930\end{array}$

$\begin{array}{llll}0.85455935 & 0.50450009 & 0.25448795\end{array}$

$\begin{array}{llll}0.02004677 & 0.19384034 & 0.40453746\end{array}$

$\begin{array}{llll}0.31321516 & 0.33401948 & 0.40556110\end{array}$

$\begin{array}{llll}0.17299878 & 0.49197112 & 0.40835564\end{array}$

$\begin{array}{llll}0.51919551 & 0.18464675 & 0.41693341\end{array}$

$\begin{array}{llll}0.82155701 & 0.33171674 & 0.41839490\end{array}$

$\begin{array}{llll}0.65915880 & 0.48821056 & 0.41476102\end{array}$

$\begin{array}{llll}0.01323108 & 0.67904796 & 0.41930491\end{array}$

$\begin{array}{llll}0.32805218 & 0.83328188 & 0.41420252\end{array}$

$\begin{array}{lll}0.17771307 & 0.98590638 & 0.41440425\end{array}$

$\begin{array}{llll}0.52087568 & 0.68939003 & 0.41711766\end{array}$

$\begin{array}{llll}0.82397931 & 0.83831296 & 0.41626464\end{array}$

$\begin{array}{llll}0.66584274 & 0.98788518 & 0.41543700\end{array}$

$\begin{array}{llll}0.48105733 & 0.32202050 & 0.57835738\end{array}$ 


\begin{tabular}{lll}
0.18474456 & 0.17245336 & 0.58028055 \\
0.33673290 & 0.01852796 & 0.58379341 \\
0.98905298 & 0.32298658 & 0.58315495 \\
0.67952626 & 0.16026410 & 0.57997147 \\
0.84621163 & 0.02188126 & 0.57516284 \\
0.49891540 & 0.81996205 & 0.58895736 \\
0.18178658 & 0.65641120 & 0.58047988 \\
0.33297575 & 0.51104889 & 0.58757634 \\
0.98733941 & 0.82653427 & 0.58214513 \\
0.68346000 & 0.66880251 & 0.58668591 \\
0.83011607 & 0.51406396 & 0.58030888 \\
0.34721297 & 0.33516840 & 0.74799303 \\
0.15582324 & 0.99605760 & 0.74821482 \\
0.00511027 & 0.15608808 & 0.75213120 \\
0.83167469 & 0.34573043 & 0.75411827 \\
0.64059538 & 0.98909285 & 0.74546537 \\
0.49236447 & 0.14503042 & 0.75190311 \\
0.34331393 & 0.84024261 & 0.74390822 \\
0.14419438 & 0.49256238 & 0.75294486 \\
0.00616798 & 0.65998558 & 0.75328172 \\
0.85288086 & 0.84651645 & 0.75404960 \\
0.65223751 & 0.51030793 & 0.74547913 \\
0.48699496 & 0.63933203 & 0.75155583 \\
0.31753326 & 0.48919474 & 0.92476623 \\
0.16697608 & 0.18339551 & 0.91976150 \\
0.01321970 & 0.32727588 & 0.91271459 \\
0.82874982 & 0.49723368 & 0.91093364 \\
0.65947646 & 0.17718396 & 0.91761114 \\
0.52316437 & 0.33494323 & 0.92164397 \\
0.31003498 & 0.98550637 & 0.91639811 \\
0.17463868 & 0.69311505 & 0.91407635 \\
0.00406753 & 0.82878304 & 0.92122822 \\
0.82307071 & 0.98890016 & 0.91328766 \\
0.65810441 & 0.67149280 & 0.92476739 \\
0.50635819 & 0.83627462 & 0.91164670 \\
0.15690796 & 0.32282756 & 0.01948422 \\
0.67832419 & 0.33731257 & 0.02179705 \\
0.17380358 & 0.83412933 & 0.01814489 \\
0.327365555 & 0.83129130 & 0.02454531 \\
& 0.99519761 & 0.14142485 \\
\hline & 0.16668574 & 0.18816620
\end{tabular}




$\begin{array}{lll}0.83913123 & 0.16444763 & 0.18845006 \\ 0.33636820 & 0.65843804 & 0.19173809 \\ 0.82072771 & 0.66368793 & 0.19348688 \\ 0.66832402 & 0.33589440 & 0.30936897 \\ 0.16890134 & 0.83075776 & 0.31520121 \\ 0.67537960 & 0.84179312 & 0.31629313 \\ 0.00694785 & 0.00541331 & 0.35288152 \\ 0.50475006 & 0.00074970 & 0.35355691 \\ 0.99772158 & 0.48698880 & 0.35243789 \\ 0.50139325 & 0.50606754 & 0.35050122 \\ 0.33329945 & 0.17668293 & 0.46947975 \\ 0.82482896 & 0.16262179 & 0.48100146 \\ 0.34650489 & 0.67761554 & 0.48285456 \\ 0.83372664 & 0.66804571 & 0.47593823 \\ 0.17027108 & 0.34075503 & 0.51993326 \\ 0.66782753 & 0.33595523 & 0.51736928 \\ 0.16466008 & 0.83203342 & 0.52462954 \\ 0.67721834 & 0.83911476 & 0.52119355 \\ 0.00374185 & 0.00628858 & 0.64485191 \\ 0.50164954 & 0.99737008 & 0.64542048 \\ 0.00337274 & 0.50919753 & 0.64506545 \\ 0.49571718 & 0.49193999 & 0.64552537 \\ 0.33867306 & 0.15953146 & 0.68364251 \\ 0.82312322 & 0.16833065 & 0.69488390 \\ 0.32674627 & 0.65684940 & 0.68580808 \\ 0.82988136 & 0.66194547 & 0.68366340 \\ 0.16357611 & 0.32357469 & 0.81558142 \\ 0.65628139 & 0.33593050 & 0.80792023 \\ 0.17424890 & 0.83502375 & 0.81460419 \\ 0.65362984 & 0.82417936 & 0.80450157 \\ 0.99617085 & 0.98960445 & 0.85902223 \\ 0.49215623 & 0.00832485 & 0.85471791 \\ 0.01068745 & 0.50149417 & 0.85804981 \\ 0.49038403 & 0.49994212 & 0.86169178 \\ 0.34324620 & 0.18163852 & 0.97561057 \\ 0.82515578 & 0.16480897 & 0.98272412 \\ 0.33207014 & 0.67348577 & 0.98745160 \\ 0.83396335 & 0.66872820 & 0.98257121 \\ 0.16522405 & 0.32737113 & 0.31647032\end{array}$

Snapshot: 31 ps (-825.513 eV)

$\begin{array}{llll}0.18006056 & 0.01245067 & 0.07880933\end{array}$

$\begin{array}{llll}0.33989920 & 0.32523205 & 0.07744120\end{array}$

$\begin{array}{llll}0.48700457 & 0.16695334 & 0.08097890\end{array}$ 


\begin{tabular}{lll}
0.68433533 & 0.02123946 & 0.08850898 \\
0.84057088 & 0.32308562 & 0.08430395 \\
0.98111164 & 0.15727412 & 0.07943470 \\
0.18986106 & 0.51920138 & 0.07975888 \\
0.34285310 & 0.82957411 & 0.08114328 \\
0.48874352 & 0.66505132 & 0.08950276 \\
0.68398829 & 0.51792618 & 0.09053541 \\
0.82529655 & 0.81031876 & 0.08191654 \\
0.97729551 & 0.65715061 & 0.08589140 \\
0.13953746 & 0.15939395 & 0.25684840 \\
0.02182729 & 0.34015540 & 0.25499992 \\
0.33769972 & 0.00475762 & 0.25303251 \\
0.65930481 & 0.15364685 & 0.24594029 \\
0.49887738 & 0.34602511 & 0.24300991 \\
0.84921119 & 0.98891375 & 0.25199935 \\
0.15511604 & 0.65084777 & 0.24935771 \\
0.99558327 & 0.85244713 & 0.24531300 \\
0.32030524 & 0.46339173 & 0.25864259 \\
0.64660920 & 0.65605237 & 0.24732660 \\
0.51104023 & 0.86159478 & 0.25457650 \\
0.83892234 & 0.48276246 & 0.24530924 \\
0.02383182 & 0.19403169 & 0.41320017 \\
0.29810534 & 0.31719426 & 0.40864477 \\
0.15709585 & 0.46775152 & 0.41706534 \\
0.52054276 & 0.18428317 & 0.41705196 \\
0.82512139 & 0.34132358 & 0.41066133 \\
0.66151708 & 0.49353043 & 0.41020566 \\
0.01309159 & 0.68400714 & 0.41504110 \\
0.31776717 & 0.83063949 & 0.41013197 \\
0.16895045 & 0.98983154 & 0.41786748 \\
0.51399275 & 0.67487233 & 0.41532002 \\
0.82033898 & 0.82901637 & 0.41084419 \\
0.65769552 & 0.98373627 & 0.42163198 \\
0.48229183 & 0.32529704 & 0.57988709 \\
0.16696675 & 0.17398101 & 0.58978970 \\
0.33403230 & 0.00544659 & 0.58383607 \\
0.98580346 & 0.31966840 & 0.58095430 \\
0.68053881 & 0.17341561 & 0.58874577 \\
0.82376907 & 0.00633035 & 0.57924914 \\
0.674785858 & 0.82298213 & 0.57766240 \\
& 0.65882948 & 0.58104101 \\
\hline
\end{tabular}




\begin{tabular}{|c|c|c|}
\hline 178 & 0.51700120 & 0 \\
\hline 0.34755156 & 0.35070959 & 0.74829056 \\
\hline 15549288 & 0.99989777 & 0.74956871 \\
\hline 9262557 & 5971933 & 5567685 \\
\hline 83952946 & 0.34252811 & 0.74929358 \\
\hline 0.64531430 & 0.99333651 & 0.75436721 \\
\hline 50505882 & 0.15493551 & 0.74506242 \\
\hline 76224 & 0.84933708 & 0.74 \\
\hline 0.14381263 & 0.49596212 & 0.74826623 \\
\hline 0.99714244 & 0.65201092 & 0.75622079 \\
\hline 85095362 & 0.85527438 & 76784 \\
\hline 0.66377923 & 0.50664407 & 0.76076986 \\
\hline 8452 & 0.64373844 & 0.74930918 \\
\hline 0.32278238 & 0.48526930 & 0.91494862 \\
\hline 17408971 & 0.18651435 & 0.91117205 \\
\hline 5760 & 0.34544979 & 4809 \\
\hline 0.82676112 & 0.49546089 & 0.92162421 \\
\hline 0.66945196 & 0.17845969 & 0.92537961 \\
\hline 0.51903307 & 0.334 & 0.918 \\
\hline 0.32627416 & 0.99093083 & 0.90934849 \\
\hline 0.17935375 & 0.68434574 & 0.91492799 \\
\hline 0.01415492 & 0.83809863 & 0.91688414 \\
\hline 0.81862258 & 0.99824 & 0. \\
\hline 0.66296404 & 0.68824233 & 0.91955509 \\
\hline 0.52189511 & 0.83748630 & 0.91997043 \\
\hline 0.16 & 0.33 & 0.0 \\
\hline 0.66625185 & 0.32845618 & 0.0 \\
\hline 0.16491106 & 0.82968766 & 0.00890480 \\
\hline 0.67257013 & 0.84160231 & 0.02545668 \\
\hline 0.99227458 & 0.98843184 & 0.14372801 \\
\hline 0.50552804 & 0.00154405 & 0.14818076 \\
\hline 0.01237656 & 0.50484443 & 0.14633122 \\
\hline 0.49828077 & 0.49889167 & 0.14776859 \\
\hline 0.31385711 & 0.15880640 & 0.18606924 \\
\hline 0.83385658 & 0.15686043 & 0.19449148 \\
\hline 0.33614993 & 0.66085363 & 0.19125099 \\
\hline 0.83143932 & 0.65645862 & 0.18716159 \\
\hline 0.65824827 & 0.32918792 & 0.30838099 \\
\hline 0.16864354 & 0.83219159 & 0.30433869 \\
\hline 0.67055918 & 0.83244082 & 0.31552006 \\
\hline 0.00814408 & 0.01058149 & 0.35495253 \\
\hline 0.49566080 & 0.00316003 & 0.35150996 \\
\hline 0.00503512 & 0.50704432 & 0.34985006 \\
\hline 0.49560952 & 0.50090776 & 0.35022513 \\
\hline
\end{tabular}




$\begin{array}{lll}0.33543376 & 0.16530457 & 0.48222163 \\ 0.83945980 & 0.17154951 & 0.47400075 \\ 0.32496491 & 0.66015833 & 0.47571523 \\ 0.83629819 & 0.67472658 & 0.47763239 \\ 0.16516510 & 0.33262624 & 0.52603915 \\ 0.66985283 & 0.34258271 & 0.51398846 \\ 0.15850960 & 0.82066156 & 0.52558891 \\ 0.66456856 & 0.82714442 & 0.51919982 \\ 0.00043006 & 0.99493017 & 0.64591135 \\ 0.50245707 & 0.00112008 & 0.64085657 \\ 0.99389523 & 0.49215165 & 0.64374793 \\ 0.50925627 & 0.49257011 & 0.65268173 \\ 0.33230224 & 0.16450330 & 0.68717642 \\ 0.82719822 & 0.16398522 & 0.68466581 \\ 0.32734963 & 0.66319629 & 0.68529808 \\ 0.82640073 & 0.66044051 & 0.69165176 \\ 0.16586227 & 0.33629221 & 0.80909570 \\ 0.66934329 & 0.33487584 & 0.81350594 \\ 0.16750083 & 0.83377036 & 0.81271642 \\ 0.66971269 & 0.82959457 & 0.81280191 \\ 0.99711324 & 0.01410119 & 0.85582890 \\ 0.50986082 & 0.00647833 & 0.85544396 \\ 0.00542198 & 0.50965348 & 0.86212093 \\ 0.49917936 & 0.50339035 & 0.85415627 \\ 0.33604649 & 0.17090463 & 0.98094298 \\ 0.83677134 & 0.17508608 & 0.98483704 \\ 0.34603299 & 0.67452214 & 0.98254695 \\ 0.82618112 & 0.66631318 & 0.98538705 \\ 0.16490989 & 0.33160367 & 0.31973322\end{array}$

Snapshot: 32.1 ps (-825.083 eV)

$\begin{array}{lll}0.17387302 & 0.00807187 & 0.08502975 \\ 0.31633780 & 0.30975980 & 0.08848070 \\ 0.48728096 & 0.16387995 & 0.08603035 \\ 0.66091352 & 0.00158549 & 0.07754973 \\ 0.83076754 & 0.31315378 & 0.08960855 \\ 0.97879119 & 0.16905961 & 0.08251423 \\ 0.17221964 & 0.51301759 & 0.08753111 \\ 0.32628861 & 0.82038254 & 0.08429942 \\ 0.48205382 & 0.67760000 & 0.07317577 \\ 0.70410142 & 0.52233548 & 0.07940515 \\ 0.83600720 & 0.81396305 & 0.07995755 \\ 0.00898097 & 0.67506113 & 0.08509027 \\ 0.15618669 & 0.17851400 & 0.25144761\end{array}$




\begin{tabular}{lll}
0.01356155 & 0.34650950 & 0.26162853 \\
0.34786192 & 0.01670784 & 0.26088647 \\
0.65099803 & 0.15408853 & 0.25054746 \\
0.51663547 & 0.34234432 & 0.24963361 \\
0.84012379 & 0.99464213 & 0.23966949 \\
0.15517784 & 0.65603415 & 0.26041672 \\
0.99724330 & 0.85908182 & 0.25960164 \\
0.32863394 & 0.48589044 & 0.25619708 \\
0.65446610 & 0.65110727 & 0.25598100 \\
0.48775846 & 0.84127740 & 0.25561762 \\
0.85073284 & 0.50976176 & 0.24892145 \\
0.01955378 & 0.18457194 & 0.40910424 \\
0.31045684 & 0.32343814 & 0.40171461 \\
0.16792254 & 0.46539519 & 0.40815345 \\
0.52140047 & 0.19061864 & 0.41484722 \\
0.81926977 & 0.33913885 & 0.42636398 \\
0.66619979 & 0.48029597 & 0.41202244 \\
0.01420895 & 0.68803350 & 0.41360852 \\
0.31134744 & 0.82604738 & 0.42151174 \\
0.16161963 & 0.98202695 & 0.41714897 \\
0.50375602 & 0.66607803 & 0.42572198 \\
0.80510418 & 0.82978042 & 0.41153104 \\
0.66496207 & 0.98445726 & 0.41325097 \\
0.48983114 & 0.32093138 & 0.58237593 \\
0.18380934 & 0.16916684 & 0.57253167 \\
0.33659398 & 0.00827266 & 0.58243995 \\
0.98754710 & 0.31738699 & 0.57771486 \\
0.67516705 & 0.15799098 & 0.58890694 \\
0.83881957 & 0.01279896 & 0.58060901 \\
0.47832920 & 0.81345427 & 0.58108436 \\
0.17099211 & 0.66668248 & 0.58559340 \\
0.32972914 & 0.50737310 & 0.57827714 \\
0.98734770 & 0.82687101 & 0.57679358 \\
0.68402989 & 0.65950562 & 0.58421343 \\
0.83325141 & 0.51675612 & 0.58375302 \\
0.35207969 & 0.34736959 & 0.75570849 \\
0.16067898 & 0.00469074 & 0.74377702 \\
0.00033565 & 0.15522880 & 0.74375453 \\
0.85776225 & 0.35211921 & 0.74588606 \\
0.66349985 & 0.00581151 & 0.75595493 \\
\hline & 0.15788987 & 0.75242543 \\
\hline
\end{tabular}




\begin{tabular}{lll}
0.84832302 & 0.84802268 & 0.74953708 \\
0.65540286 & 0.50269072 & 0.75015824 \\
0.50336341 & 0.64390800 & 0.74605165 \\
0.33195339 & 0.49392057 & 0.92067750 \\
0.16814782 & 0.17282649 & 0.91907549 \\
0.01193246 & 0.32343498 & 0.91589579 \\
0.83378283 & 0.49285250 & 0.91515821 \\
0.66688982 & 0.18904527 & 0.91432954 \\
0.52105989 & 0.32847711 & 0.92182392 \\
0.33182230 & 0.98539105 & 0.91853591 \\
0.16829984 & 0.68455902 & 0.90939705 \\
0.02053996 & 0.83472935 & 0.91134387 \\
0.81900442 & 0.98288579 & 0.91471611 \\
0.67770006 & 0.68595428 & 0.91467524 \\
0.51168915 & 0.83680887 & 0.91289015 \\
0.16490248 & 0.33809652 & 0.02382801 \\
0.66834676 & 0.34152897 & 0.02456601 \\
0.16134350 & 0.82991532 & 0.01754831 \\
0.67035348 & 0.83792655 & 0.01767435 \\
0.99979407 & 0.00238899 & 0.14715775 \\
0.50388770 & 0.99919349 & 0.14345822 \\
0.00327421 & 0.51057414 & 0.15272356 \\
0.49161255 & 0.49865713 & 0.13870137 \\
0.33067832 & 0.17929185 & 0.19072050 \\
0.83832605 & 0.17005901 & 0.19562187 \\
0.33261071 & 0.67517508 & 0.18523600 \\
0.83544710 & 0.67630049 & 0.18768180 \\
0.66419588 & 0.32713691 & 0.31574017 \\
0.16294379 & 0.83716071 & 0.32058612 \\
0.65921253 & 0.82624237 & 0.30846252 \\
0.98270384 & 0.99320521 & 0.35795993 \\
0.49409209 & 0.99682952 & 0.35455923 \\
0.00723685 & 0.50133832 & 0.35867701 \\
0.49544499 & 0.48678055 & 0.34602705 \\
0.33628847 & 0.16721473 & 0.47187208 \\
0.83939832 & 0.16767669 & 0.47833278 \\
0.33705401 & 0.66535780 & 0.48061808 \\
0.83399677 & 0.66789464 & 0.47453814 \\
0.16011944 & 0.32731777 & 0.51720905 \\
0.46614232 & 0.33362323 & 0.52534544 \\
\hline & 0.83099658 & 0.51956014 \\
\hline
\end{tabular}




$\begin{array}{lll}0.99502162 & 0.50704181 & 0.64628775 \\ 0.49585956 & 0.49919228 & 0.64066246 \\ 0.33945644 & 0.17490173 & 0.67941665 \\ 0.83459804 & 0.16403734 & 0.69311583 \\ 0.33212621 & 0.65412000 & 0.68665457 \\ 0.84080685 & 0.67411731 & 0.68337085 \\ 0.16765744 & 0.32379246 & 0.82222737 \\ 0.66697738 & 0.34170624 & 0.81897144 \\ 0.16758016 & 0.84130226 & 0.81163994 \\ 0.66809210 & 0.82629396 & 0.80902370 \\ 0.99851970 & 0.99899840 & 0.85709313 \\ 0.50752721 & 0.00692893 & 0.85302255 \\ 0.00730087 & 0.49518733 & 0.85415781 \\ 0.49878562 & 0.49289811 & 0.86036693 \\ 0.32737691 & 0.15642753 & 0.98439754 \\ 0.81780729 & 0.15718324 & 0.98292883 \\ 0.33676121 & 0.66745884 & 0.97577798 \\ 0.84193460 & 0.66568318 & 0.97364223 \\ 0.16686940 & 0.33133414 & 0.31410652\end{array}$

Snapshot: 38.2 ps (-825.484 eV)
$\begin{array}{llll}0.18064406 & 0.02310682 & 0.08230953\end{array}$
$\begin{array}{llll}0.34371117 & 0.31415881 & 0.08361350\end{array}$
$\begin{array}{llll}0.49450812 & 0.15883027 & 0.08234213\end{array}$
$\begin{array}{llll}0.66834596 & 0.00785694 & 0.07943147\end{array}$
$\begin{array}{llll}0.83044036 & 0.31672831 & 0.08702624\end{array}$
$\begin{array}{llll}0.98385991 & 0.16602526 & 0.08059836\end{array}$
$\begin{array}{llll}0.18079626 & 0.50652680 & 0.09126147\end{array}$
$\begin{array}{llll}0.32825339 & 0.81399406 & 0.08474420\end{array}$
$\begin{array}{llll}0.48517487 & 0.67547051 & 0.08617529\end{array}$
$\begin{array}{llll}0.68259634 & 0.52340163 & 0.07433377\end{array}$
$\begin{array}{llll}0.84008557 & 0.82670162 & 0.07793837\end{array}$
$\begin{array}{llll}0.99616444 & 0.67038735 & 0.06912334\end{array}$
$\begin{array}{llll}0.15525580 & 0.18092050 & 0.25718671\end{array}$
$\begin{array}{llll}0.01056700 & 0.34626445 & 0.25675574\end{array}$
$\begin{array}{llll}0.33750918 & 0.99620886 & 0.25217978\end{array}$
$\begin{array}{llll}0.64454913 & 0.14694670 & 0.25469622\end{array}$
$\begin{array}{llll}0.51266875 & 0.35172576 & 0.24655491\end{array}$
$\begin{array}{llll}0.84075015 & 0.99822037 & 0.25279366\end{array}$
$\begin{array}{llll}0.15720569 & 0.65724451 & 0.24926199\end{array}$
$\begin{array}{llll}0.00720340 & 0.85252990 & 0.25128430\end{array}$
$\begin{array}{llll}0.32506879 & 0.47646718 & 0.25676938\end{array}$
$\begin{array}{lll}0.64252777 & 0.64101382 & 0.25970816\end{array}$
$\begin{array}{llll}0.49856233 & 0.85111446 & 0.25409946\end{array}$ 


\begin{tabular}{lll}
0.84209669 & 0.48857115 & 0.24739214 \\
0.02899046 & 0.20579564 & 0.41644675 \\
0.31288132 & 0.33353288 & 0.41101112 \\
0.17150246 & 0.47861214 & 0.41020791 \\
0.51456456 & 0.17639852 & 0.42270199 \\
0.82097861 & 0.33228654 & 0.41623961 \\
0.65976465 & 0.47635621 & 0.41557678 \\
0.00966646 & 0.68194197 & 0.41744135 \\
0.31724785 & 0.83433240 & 0.42090400 \\
0.16215857 & 0.99104514 & 0.42278138 \\
0.51417049 & 0.67638544 & 0.41917622 \\
0.82674964 & 0.83918551 & 0.42265143 \\
0.65616979 & 0.98030902 & 0.42523751 \\
0.48792710 & 0.32295621 & 0.58193991 \\
0.18506133 & 0.16503373 & 0.58317720 \\
0.33538826 & 0.01399627 & 0.58156120 \\
0.99386644 & 0.32399911 & 0.58343643 \\
0.68726605 & 0.17806813 & 0.59441797 \\
0.82899458 & 0.01617324 & 0.58175458 \\
0.48306027 & 0.81777021 & 0.58627650 \\
0.16427218 & 0.65802465 & 0.57239074 \\
0.33499344 & 0.52141161 & 0.58297871 \\
0.98316754 & 0.82539714 & 0.58838748 \\
0.67887933 & 0.66645586 & 0.57799588 \\
0.82963894 & 0.50895111 & 0.58829496 \\
0.34534932 & 0.34373695 & 0.74946312 \\
0.15894260 & 0.00645394 & 0.75025810 \\
0.00595393 & 0.14800530 & 0.74934281 \\
0.84831679 & 0.33570963 & 0.75763352 \\
0.65072160 & 0.99836811 & 0.74615608 \\
0.49429594 & 0.14999144 & 0.74062205 \\
0.34876414 & 0.84645127 & 0.75283146 \\
0.15506110 & 0.50108324 & 0.74776521 \\
0.98426422 & 0.64548129 & 0.75775322 \\
0.85050612 & 0.85295910 & 0.74431157 \\
0.65226275 & 0.49703431 & 0.75102391 \\
0.49154856 & 0.64620160 & 0.74917337 \\
0.32826446 & 0.49004476 & 0.92201015 \\
0.17356411 & 0.19009599 & 0.92044853 \\
0.01730068 & 0.33412892 & 0.92698243 \\
\hline & 0.48404399 & 0.91254151 \\
\hline
\end{tabular}




\begin{tabular}{lll}
0.16224429 & 0.65966178 & 0.90863724 \\
0.02790147 & 0.83334908 & 0.91620137 \\
0.83211503 & 0.98721016 & 0.91681792 \\
0.66746733 & 0.67797291 & 0.90814450 \\
0.52312202 & 0.83055608 & 0.91818414 \\
0.17115881 & 0.33538251 & 0.01851121 \\
0.66123174 & 0.33447182 & 0.01982542 \\
0.17066873 & 0.84024956 & 0.02213256 \\
0.66256416 & 0.82392326 & 0.02516626 \\
0.99305604 & 0.99184186 & 0.14475669 \\
0.49751906 & 0.99382953 & 0.14805904 \\
0.00139143 & 0.50115410 & 0.13929981 \\
0.51007018 & 0.51328401 & 0.15063294 \\
0.34031603 & 0.17659679 & 0.19204848 \\
0.83310049 & 0.16451359 & 0.19224992 \\
0.32986093 & 0.66121698 & 0.18894913 \\
0.83030975 & 0.66251227 & 0.19095918 \\
0.67081710 & 0.32720886 & 0.31461266 \\
0.16407196 & 0.83553607 & 0.31082995 \\
0.66391283 & 0.82702830 & 0.31267807 \\
0.00405639 & 0.00493413 & 0.35389517 \\
0.50257236 & 0.99916830 & 0.35679888 \\
0.99848014 & 0.50056016 & 0.34759617 \\
0.49489160 & 0.49367845 & 0.35581963 \\
0.34308224 & 0.17113309 & 0.48009816 \\
0.83516790 & 0.17047086 & 0.48380362 \\
0.32803533 & 0.67561116 & 0.48364335 \\
0.82657977 & 0.65758178 & 0.47303032 \\
0.16973059 & 0.32933410 & 0.51883308 \\
0.66077056 & 0.33179865 & 0.52787842 \\
0.15719622 & 0.84237453 & 0.52158649 \\
0.66313004 & 0.83158781 & 0.52083990 \\
0.00154032 & 0.00938618 & 0.63719529 \\
0.50873975 & 0.00502075 & 0.63997234 \\
0.99866043 & 0.50030566 & 0.65066913 \\
0.49071289 & 0.50224461 & 0.64243182 \\
0.33268605 & 0.16976851 & 0.68584759 \\
0.84395687 & 0.16571746 & 0.68841969 \\
0.33294884 & 0.66640381 & 0.68898789 \\
0.82216302 & 0.67221206 & 0.68269639 \\
\hline & 0.32594579 & 0.81308755 \\
\hline
\end{tabular}




$\begin{array}{lll}0.01526061 & 0.00835902 & 0.85456164 \\ 0.51013098 & 0.99550142 & 0.85486066 \\ 0.99134444 & 0.49210686 & 0.85534043 \\ 0.50421278 & 0.51023061 & 0.84703962 \\ 0.34416782 & 0.16254203 & 0.97779718 \\ 0.83559273 & 0.16700232 & 0.97867537 \\ 0.32681114 & 0.66543792 & 0.97480223 \\ 0.83765843 & 0.66212396 & 0.97986847 \\ 0.16469591 & 0.34049979 & 0.31786705\end{array}$

